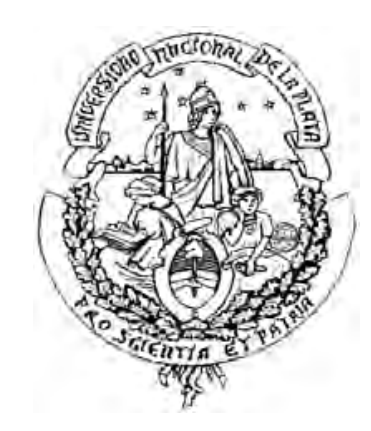

\title{
"Estudio teórico de pequeños agregados binarios constituidos por elementos de los grupos III y V'
}

\author{
Tesis Doctoral
}

Verónica Ferraresi Curotto

Director: Dr. Reinaldo Pis Diez

Departamento de Química

Facultad de Ciencias Exactas Universidad Nacional de La Plata 
Ferraresi Curotto, Verónica

Estudio teórico de pequeños agregados binarios constituidos por elementos de los grupos III y V. - 1a ed. - La Plata : Universidad Nacional de La Plata, 2012.

E-Book.

ISBN 978-950-34-0854-4

1. Química. 2. Enseñanza Universitaria. I. Título CDD 540.711

Fecha de catalogación: 31/05/2012 


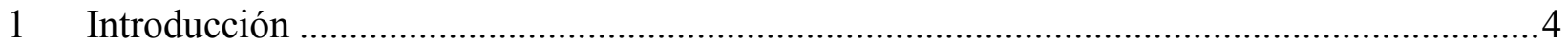

$1.1 \quad$ Preparación de semiconductores III/V nanocristalinos .................................................... 4

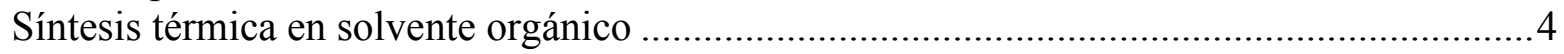

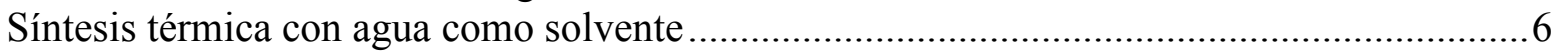

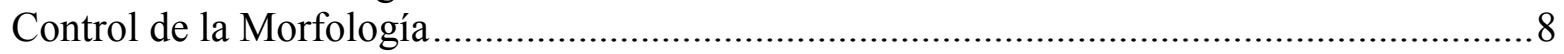

1.2 Reactividad química de nanocristales de GaP en síntesis orgánica .................................10

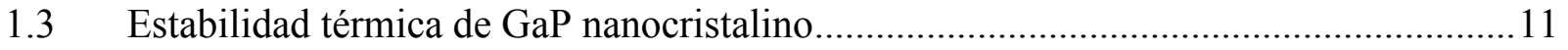

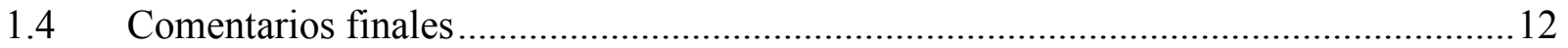

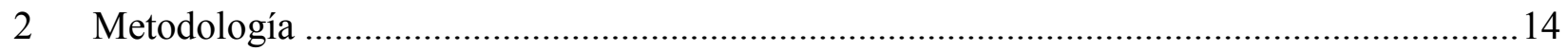

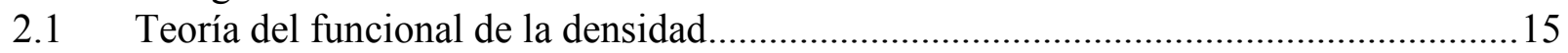

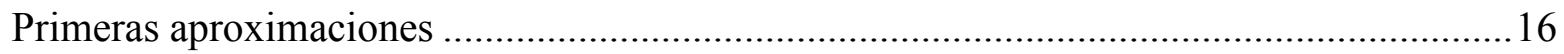

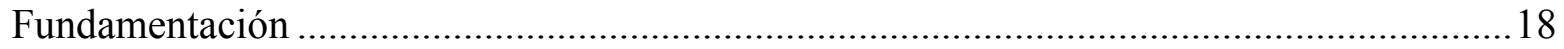

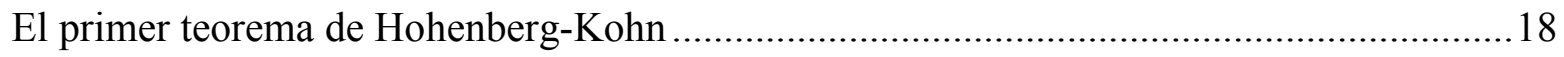

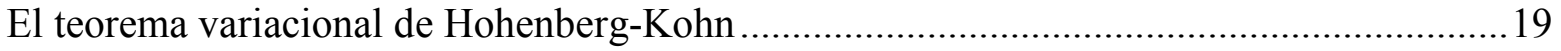

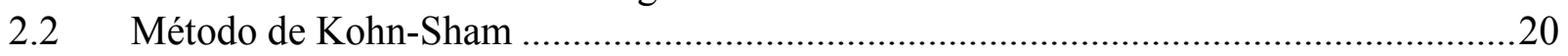

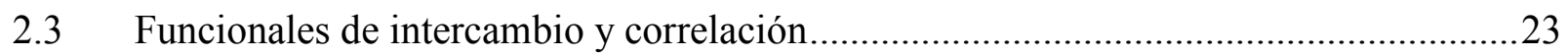

Aproximación local de la densidad ..................................................................................2

Funcionales que incluyen correcciones por gradiente de la densidad y funcionales híbridos 27

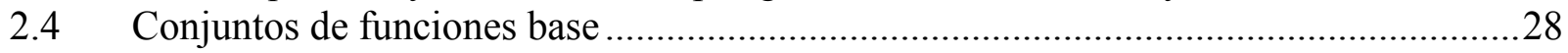

Funciones base tipo Slater y gausianas ………………...................................................2

2.5 Análisis poblacional basado en el potencial electrostático molecular ............................33

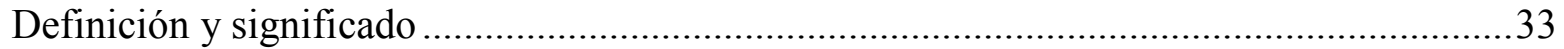

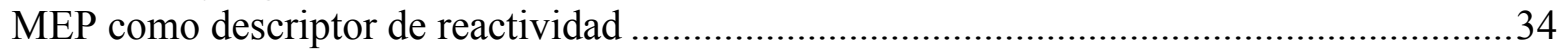

Limitaciones del uso del potencial electrostático como indicador de reactividad ...................35

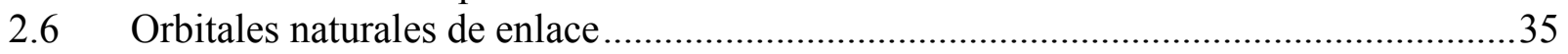

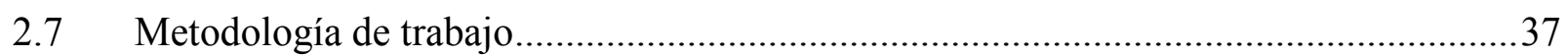

3 Estudio de la estructura, modos vibracionales y patrones de crecimiento de clusters de

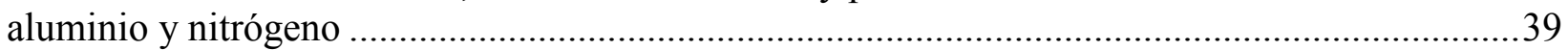

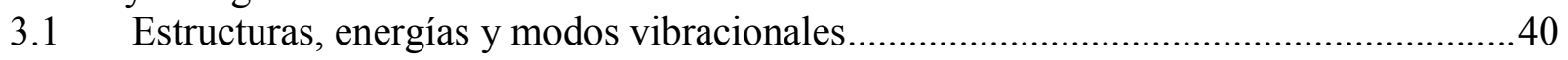

3.2 Potenciales electrostáticos moleculares ......................................................................5

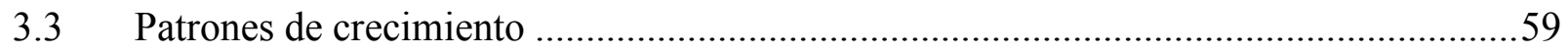

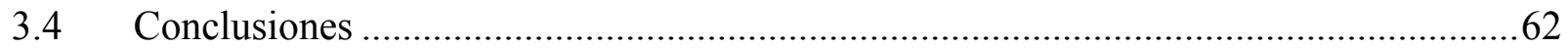

4 Estudio de la estructura, modos vibracionales y patrones de crecimiento de clusters de boro

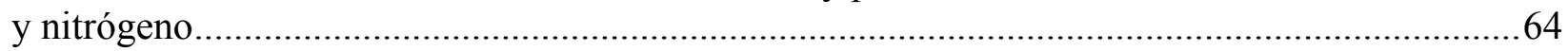

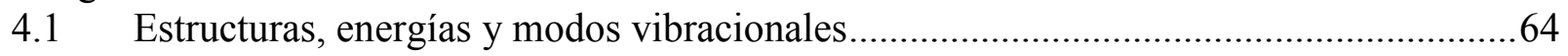

4.2 Potenciales electrostáticos moleculares .......................................................................

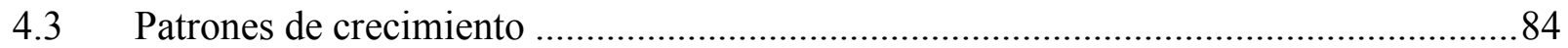

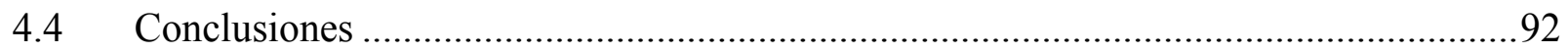

5 Estudio de la estructura, modos vibracionales y patrones de crecimiento de clusters de

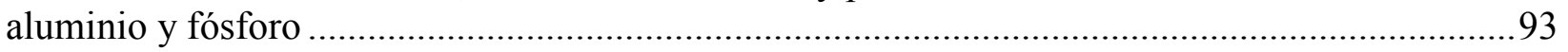

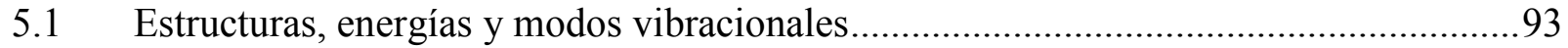

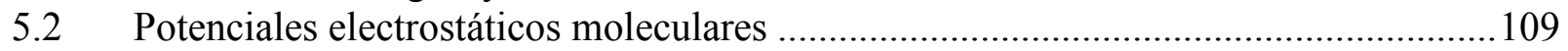

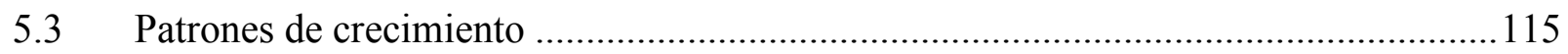

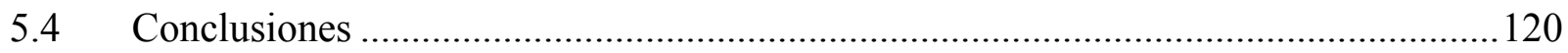

6 Estudio de la estructura, modos vibracionales y patrones de crecimiento de clusters de boro

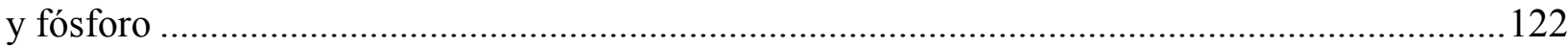

6.1 Estructuras, energías y modos vibracionales..............................................................122 


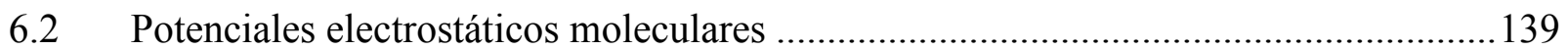

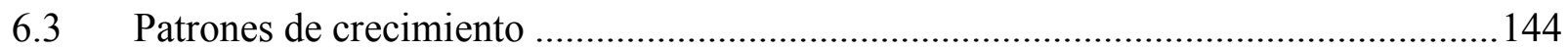

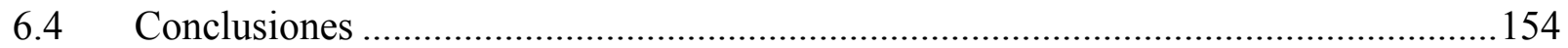

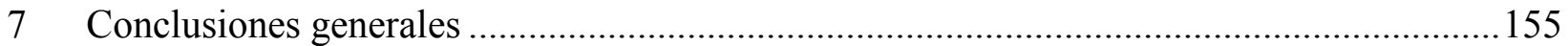

APÉNDICE 1: Teoría del funcional de la densidad conceptual ..................................................156

A. Potencial químico electrónico y electronegatividad...........................................................156

a) Esquema general: derivadas de la energía y funciones de respuesta en el ensamble

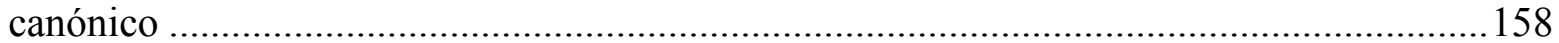

B. Función de localización electrónica (ELF, electron localization function) ......................161

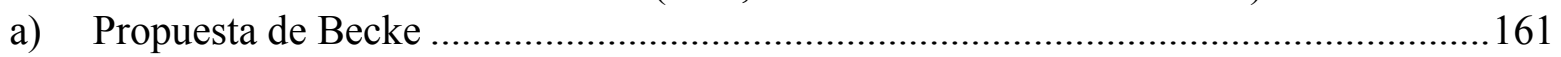

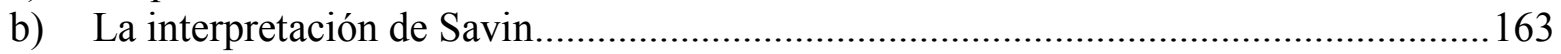

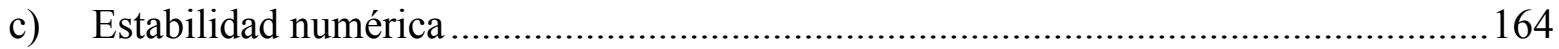

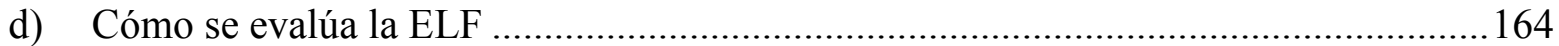

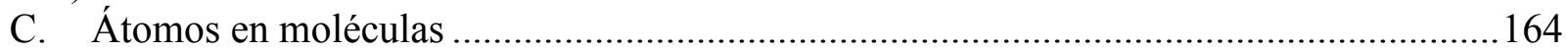

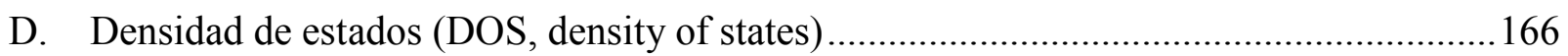

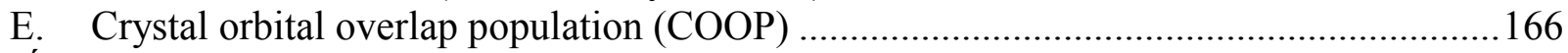

APÉNDICE 2: Análisis preliminares de agregados de Al/N .......................................................168

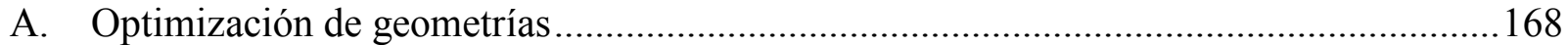

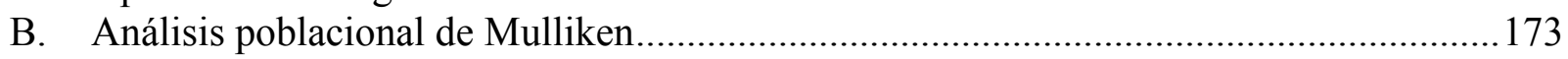

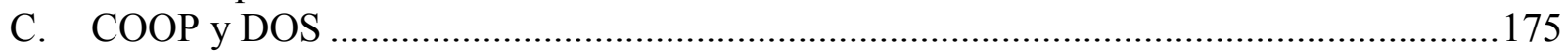

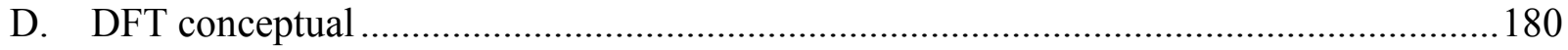

a) Afinidad electrónica, potencial de ionización, dureza y electronegatividad .................180

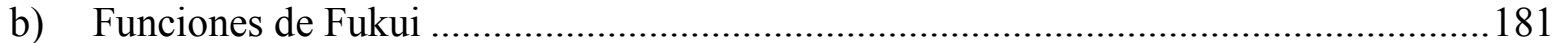

Conclusiones del análisis preliminar de agregados de $\mathrm{Al} / \mathrm{N}$.................................................182

APÉNDICE 3: Análisis preliminares de agregados de B/N: el uso de distintos métodos de análisis

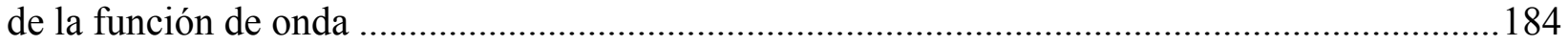

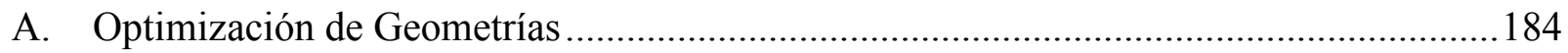

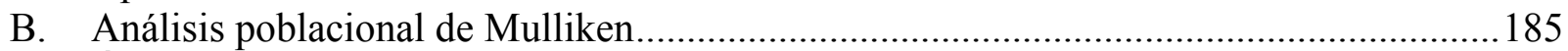

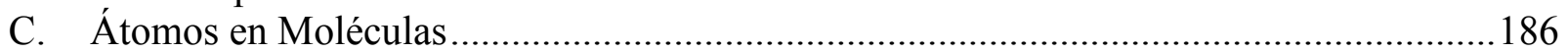

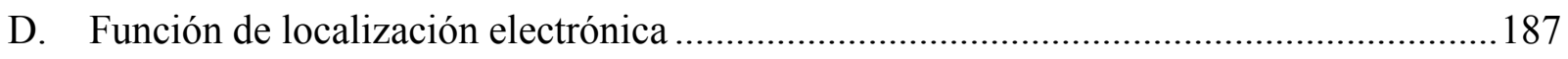

Conclusiones del análisis preliminar para agregados de $\mathrm{B} / \mathrm{N}$................................................187 


\section{Introducción}

Los compuestos constituidos por elementos de los grupos III/V, al ser destacados semiconductores, han sido objeto de intensas investigaciones tanto experimentales como teóricas debido a sus aplicaciones en catálisis y en la fabricación de dispositivos a nanoescala. Sin embargo, la obtención de estos agregados de manera experimental no es tarea fácil. Algunas técnicas de preparación requieren tecnologías de deposición de vapor a temperaturas cercanas a los $1000{ }^{\circ} \mathrm{C}^{1}$. Por ello, una meta importante es la preparación de compuestos III/V estables por métodos físico-químicos en condiciones menos extremas. En este capítulo se revisa la preparación, reactividad y estabilidad térmica de compuestos de la familia, siendo GaN el más estudiado, con el objeto de introducir en el tema en estudio y exponer la importancia de conocer las propiedades de los agregados III/V.

\subsection{Preparación de semiconductores III/V nanocristalinos}

\section{Síntesis térmica en solvente orgánico}

Nitruros: GaN, InN, AlN

Los nitruros del grupo IIIA han sido objeto de numerosos estudios para su caracterización y síntesis, debido a sus extraordinarias propiedades físicas y sus potenciales aplicaciones como materiales optoelectrónicos en las regiones verde, azul y ultravioleta. Su resistencia a la radiación y conductividades térmicas altas, entre otras propiedades, han llevado a su investigación en el posible uso en ambientes hostiles.

La mayoría de los estudios se centran en GaN hexagonal con estructura de wurtzita, que es un semiconductor muy conocido por sus propiedades en dispositivos emisores de luz en la región ultravioleta visible. A temperatura ambiente, GaN cristaliza usualmente en estructura de tipo wurtzita, sin embargo, se encuentran dos estructuras metaestables de GaN: halita y esfalerita. El GaN de tipo esfalerita se forma durante el proceso de preparación de películas delgadas, mientras que la estructura de tipo halita sólo aparece utilizando presiones muy altas. De hecho, desde el punto de vista estructural, aplicar presiones altas puede dar como resultado una serie de transformaciones de los compuestos III/V desde cristales de wurzita a cristales de tipo halita. El GaN de tipo halita es una fase metaestable con características muy diferentes con respecto a las estructuras esfalerita o wurtzita. Por ejemplo, la estructura de tipo halita está característicamente constituida por uniones iónicas, mientras que las estructuras de tipo esfalerita y wurtzita poseen propiedades parcialmente iónicas y covalentes. InN y $\mathrm{AlN}$ son casos análogos a GaN. Por ejemplo, la estructura halita de AIN también puede obtenerse aplicando altas presiones.

A pesar de que los métodos convencionales de síntesis existen y son ampliamente utilizados, una ruta basada en solvente es una alternativa atractiva para la síntesis de nitruros del grupo IIIA, bajo condiciones que resulten más amigables. Dentro de estas alternativas, han habido esfuerzos para sintetizar nitruros a altas temperaturas y con benceno como solvente. Xie y colaboradores prepararon partículas de nitruro de galio $(\mathrm{GaN})$ por reacción térmica de $\mathrm{Li}_{3} \mathrm{~N}$ y $\mathrm{GaCl}_{3}$ en la cual el benceno fue utilizado como solvente, bajo presión y a $280-300{ }^{\circ} \mathrm{C}^{1}$. Ésta temperatura es 
mucho menor que la utilizada en métodos tradicionales y se obtuvo una pureza del $80 \%$. El análisis de difracción por rayos $\mathrm{X}$ del polvo indicó que se trataba de la fase hexagonal del GaN con una pequeña porción de halita. Los cristales tenían un tamaño promedio de $32 \mathrm{~nm}$ y exhibían forma uniforme. Aunque sólo el $10 \%$ de la muestra resultó de tipo halita, les dio a los autores la posibilidad de que este tipo de GaN podía ser obtenido mediante una ruta apropiada de síntesis y podría existir en condiciones ambientales. El procedimiento para lograr la síntesis de GaN halita se basó en dos hipótesis: llevar a cabo la reacción bajo condiciones menos extremas por un período de tiempo mayor sería más favorable para obtener la fase metaestable, y la fuente de nitruro con ionicidad mayor favorecería la formación del nitruro metálico ${ }^{2}$. La reacción se basa en la reacción térmica en solvente del haluro metálico $\left(\mathrm{GaX}_{3}\right)$ y nitruros de metales alcalinos $\left(\mathrm{M}_{3} \mathrm{~N}\right)$. La fuente de nitrógeno utilizada fue amida de sodio $\left(\mathrm{NaNH}_{2}\right)$, de la cual se conoce que se transforma a $\mathrm{Na}_{3} \mathrm{~N}$ por pérdida de amoníaco bajo calentamiento. La alta reactividad del nitruro lo convierte en una buena fuente:

$3 \mathrm{NaNH}_{2} \rightarrow \mathrm{Na}_{3} \mathrm{~N}+2 \mathrm{NH}_{3}$

$\mathrm{GaI}_{3}+\mathrm{Na}_{3} \mathrm{~N} \rightarrow \mathrm{GaN}+3 \mathrm{NaI}$

Asimismo, la liberación de amoniaco por (1.1), incrementa la presión rápidamente, lo que también sería de ayuda para la formación de GaN de tipo halita. Otra consideración a tener en cuenta es que dentro de los haluros de $\mathrm{Ga}, \mathrm{GaI}_{3}$ es el mejor candidato dado que tiene la unión Ga$\mathrm{X}$ más débil, lo que garantiza la reacción de $\mathrm{GaX}_{3}$ y $\mathrm{Na}_{3} \mathrm{~N}$. También se adicionó $\mathrm{I}_{2}$ para que actúe como agente de transporte, ayudando al producto $\mathrm{NaI}$ a disolverse en benceno en la forma de $\mathrm{NaI}_{3}$, lo que completa la reacción:

$\mathrm{I}_{2}+\mathrm{NaI} \rightarrow \mathrm{NaI}_{3}$

Además, la temperatura, el tiempo y la relación molar entre los reactivos también se ajustaron. Estas optimizaciones sistemáticas del método llevaron a los autores a un progreso significativo en la preparación de $\mathrm{GaN}$ nanocristalino de alta pureza y calidad.

Otro nitruro de importancia tecnológica es el $\mathrm{InN}$, por sus propiedades ópticas y de transporte prometedoras $^{3}$. Una celda solar de InN puede, teóricamente, alcanzar una eficiencia de $32.1 \%{ }^{4}$, lo cual es altamente deseable para aplicaciones de energía solar. Sin embargo, dentro de los nitruros IIIA, el crecimiento de InN es el más difícil de lograr, debido a su baja temperatura de descomposición $\left(427-550{ }^{\circ} \mathrm{C}\right)^{5}$, lo que hace difícil el crecimiento de este nitruro. El crecimiento de InN es importante debido a que si se permite a este nitruro intercalarse entre agregados de AlN o GaN el gap del semiconductor podrá disminuirse y hará a estos materiales más conductores. Los mismos autores han extendido el método de síntesis térmica con benceno a éste y otros nitruros IIIA. La fuente de indio utilizada fue $\mathrm{In}_{2} \mathrm{~S}_{3}$, sin modificar la fuente de $\mathrm{Na}_{3} \mathrm{~N}$. Sustituyendo $\mathrm{In}_{2} \mathrm{~S}_{3}$ por $\mathrm{Al}_{2} \mathrm{~S}_{3}$ o $\mathrm{Ga}_{2} \mathrm{~S}_{3}$, se obtienen $\mathrm{AlN}$ y GaN.

\section{Fosfuros: GaP, InP}

Las rutas de obtención mediante precursores orgánicos y metálicos han sido las formas más importantes para preparar nanoestructuras de GaP amorfas o critalinas. Dougall y colaboradores ${ }^{6}$ sintetizaron agregados de semiconductores GaP mediante la reacción:

$\left(\mathrm{CH}_{3}\right)_{3} \mathrm{Ga}+\mathrm{PH}_{3} \rightarrow 3 \mathrm{CH}_{4}+\mathrm{GaP}$ 
En otros estudios, nanocristales de $\mathrm{GaP}$ se obtuvieron a partir de $\mathrm{GaCl}_{3}$ y $\mathrm{P}\left(\mathrm{SiMe}_{3}\right)_{3}$ o $(\mathrm{Na} / \mathrm{K})_{3} \mathrm{P}$ y $\mathrm{GaX}_{3}$ en tolueno u otro solvente, respectivamente ${ }^{7,8}$. Otro método a destacar para la preparación de GaP es el del estado sólido, en el cual se utilizan un haluro $\mathrm{GaX}_{3}$ y $\mathrm{NaP}_{3}$ como reactivos ${ }^{9,10}$, pero éste método requiere lavados adicionales para remover materiales precursores y productos secundarios. Se puede decir que la preparación de compuestos semiconductores III/V es una tarea ardua, debido a que los materiales precursores utilizados con frecuencia son tóxicos y peligrosos. Gao y coautores ${ }^{11}$ exploraron la síntesis de nanocristales de $\mathrm{GaP}$ de tipo esfalerita utilizando benceno como solvente y a $240-300{ }^{\circ} \mathrm{C}$. Na, P (rojo) y $\mathrm{GaCl}_{3}$ se utilizaron como reactivos.

Con el objeto de usar reactivos poco costosos y procedimiento sencillos, $\mathrm{KBH}_{4}$ y sus derivados han sido utilizados para preparar polvos metálicos y aleaciones de InP por medio de la reducción de sales metálicas en solventes orgánicos ${ }^{12}$, siendo extendido posteriormente para la preparación de compuestos, más que elementos o aleaciones, ${ }^{13}$

$4 \mathrm{InCl}_{3}+12 \mathrm{KBH}_{4}+\mathrm{P}_{4} \rightarrow 4 \mathrm{InP}+12 \mathrm{KCl}+12 \mathrm{BH}_{3}+6 \mathrm{H}_{2}$

La reacción (1.5) se llevó a cabo en etilendiamina, la que juega un rol importante en la formación de nanocristales de InP. Los experimentos demostraron que puede incrementar el área superficial de los reactivos y promover la reacción a bajas temperaturas, dispersando al fósforo amarillo en una dispersión marrón y luego disolviendo rápidamente $\mathrm{KBH}_{4}$. Cuando se utilizó benceno como solvente, la reacción no tuvo lugar, debido a la baja solubilidad de $\mathrm{KBH}_{4}$ en benceno.

\section{Síntesis térmica con agua como solvente}

\section{Fosfuros (GaP, InP)}

En los últimos años se han realizado esfuerzos considerables para explorar nuevas rutas hacia la obtención de semiconductores III/ $\mathrm{V}^{14,15}$. Con el objeto de encontrar mejores condiciones para su preparación, se ha logrado disminuir la temperatura, así también como evadir reacciones complejas y precursores tóxicos. Sin embargo, ha sido muy dificultoso preparar semiconductores $\mathrm{III} / \mathrm{V}$ en solución acuosa, mayormente debido a la hidrólisis fuerte de $\mathrm{GaX}_{3}, \mathrm{InX}_{3}(\mathrm{x}=\mathrm{Cl}, \mathrm{Br}, \mathrm{I})$, y sus compuestos organometálicos. Sin embargo, desde el punto de vista de la química verde, el agua es el medio ideal para la ruta de obtención de semiconductores III/V.

Gao y colaboradores ${ }^{16}$ presentaron la primera preparación de nanocristales de GaP y InP. La reacción se lleva a cabo en solución acuosa, a $120-160{ }^{\circ} \mathrm{C}$ e involucra el transporte de fósforo por $\mathrm{I}_{2}$ y entre $\mathrm{H}_{3} \mathrm{PO}_{4}$ y $\mathrm{H}_{3} \mathrm{PO}_{3}$. Todo el proceso se muestra en la Figura 1.1, tomando como ejemplo al $\mathrm{GaP}$. Los autores adicionaron iodo para acelerar la dismutación del fósforo blanco e inducir otra reacción circular entre $\mathrm{H}_{3} \mathrm{PO}_{4} \mathrm{y} \mathrm{H}_{3} \mathrm{PO}_{3}$. Se obtuvieron nanocristales de $\mathrm{GaP}$ y InP de tipo halita. 


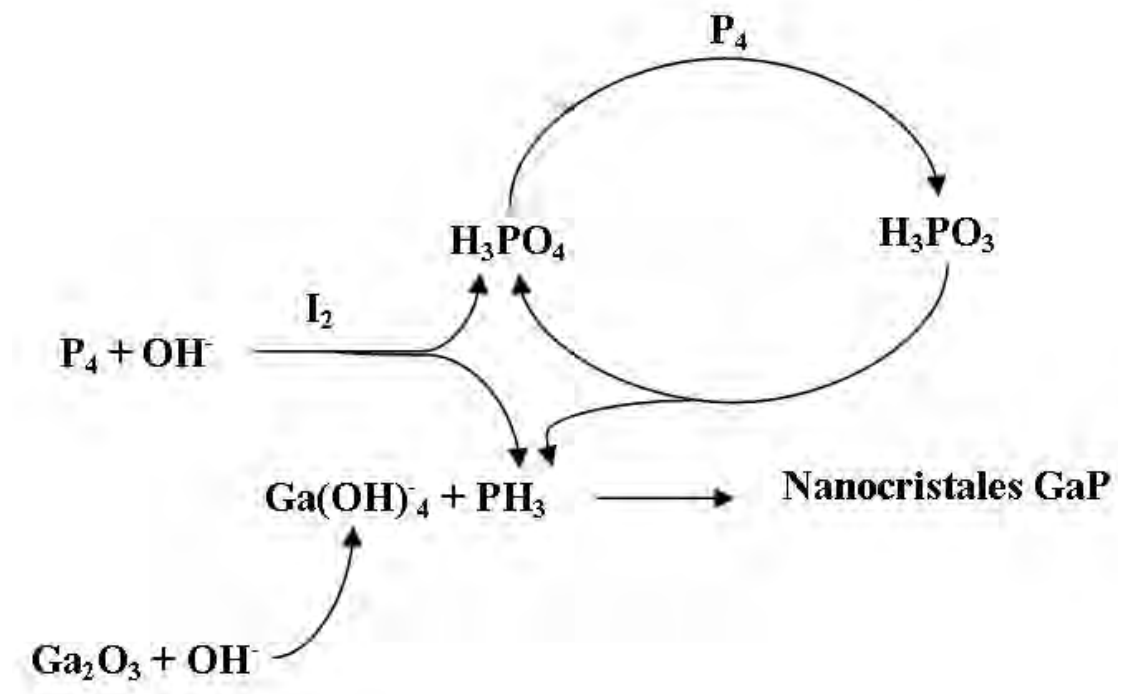

Figura 1.1. Diagrama de obtención de GaP nanocristalino.

Nitruros $(\mathrm{GaN}, \operatorname{InN})$

Xiong y coautores ${ }^{17}$ sintetizaron exitosamente nitruros del grupo IIIA en solución acuosa y a 250 ${ }^{\circ} \mathrm{C}$. La ruta seguida fue la siguiente:

$\mathrm{NH}_{4} \mathrm{Cl} \rightarrow \mathrm{NH}_{3}+\mathrm{HCl}$

$\mathrm{CS}_{2}+2 \mathrm{NH}_{3} \rightarrow \mathrm{NH}_{4} \mathrm{SCN}+\mathrm{H}_{2} \mathrm{~S}$

$2 \mathrm{NH}_{4} \mathrm{SCN} \rightarrow \mathrm{NH}: \mathrm{C}\left(\mathrm{NH}_{2}\right)_{2}+\mathrm{NH}_{3} \mathrm{CS}_{2}$

$\mathrm{In}_{2} \mathrm{~S}_{3}+3 \mathrm{NH}: \mathrm{C}\left(\mathrm{NH}_{2}\right)_{2} \rightarrow \mathrm{In}_{2}(\mathrm{NH})_{3}+3\left(\mathrm{NH}_{2}\right)_{2} \mathrm{CS}$

$\mathrm{In}_{2}(\mathrm{NH})_{3} \rightarrow 2 \mathrm{InN}+\mathrm{NH}_{3}$

Sin embargo, el $\operatorname{In}_{2}(\mathrm{NH})_{3}$ producido se hidroliza con facilidad, por lo que la cantidad que participa en (1.10) es baja y se obtiene un rendimiento del 5\% de InN. Con el objeto de incrementar la cantidad de $\mathrm{InN}$ obtenido, los autores agregaron iodo para oxidar el $\operatorname{In}_{2}(\mathrm{NH})_{3}$ producido en la reacción (1.9),

$2 \mathrm{In}_{2}(\mathrm{NH})_{3}+3 \mathrm{I}_{2} \rightarrow 4 \mathrm{InN}+\mathrm{N}_{2}+6 \mathrm{HI}$

La adición de yodo mejoró el rendimiento del método a un 25\%. Como resultado, la síntesis acuosa descripta se diseñó para la obtención de nitruros del grupo IIIA (InN, GaN y AIN), como se presenta en la Figura 1.2 para el ejemplo del InN. Esta ruta también puede ser extendida para la obtención de aleaciones de InGaN. 


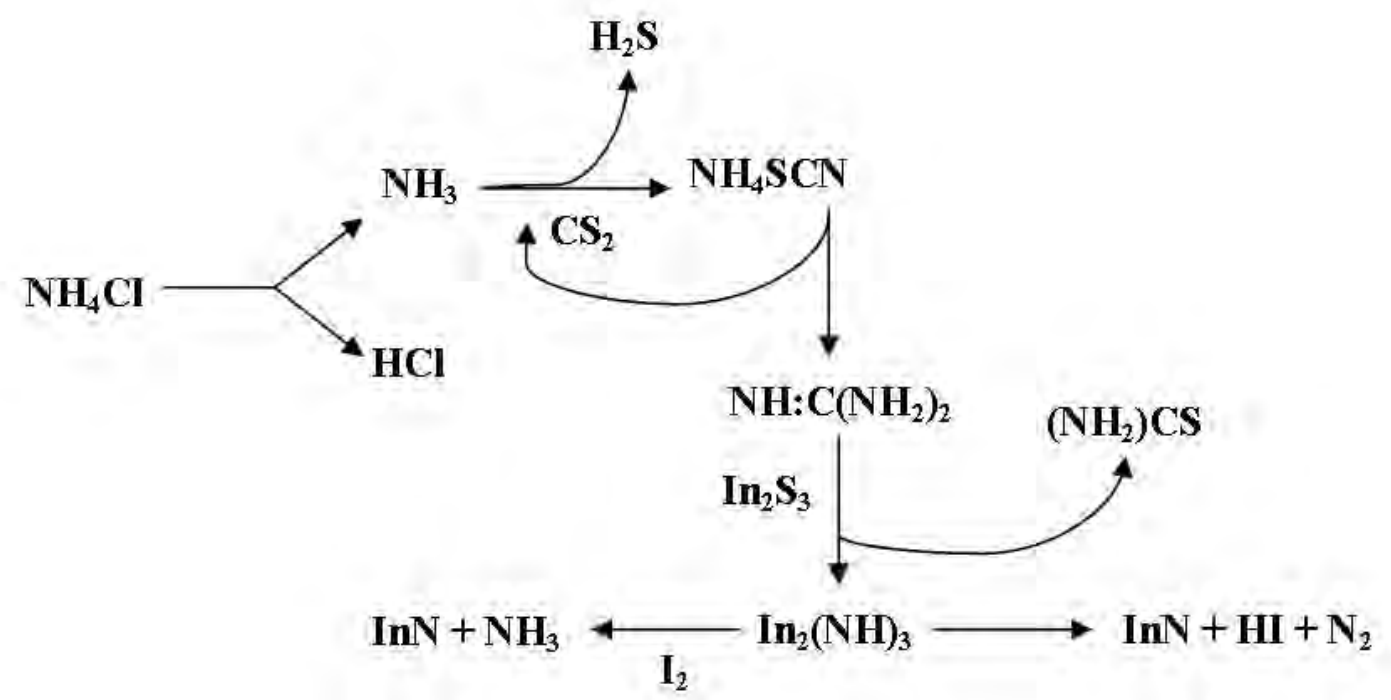

Figura 1.2. Diagrama de obtención de InN.

\section{Control de la Morfología}

La morfología del material obtenido es un factor fundamental que puede influenciar significativamente sus propiedades y aplicaciones. Recientemente, se ha puesto mucho énfasis en el control de la morfología de los nanomateriales ${ }^{18}$. Asimismo, es de amplio conocimiento que las películas delgadas de los semiconductores III/V son materiales prometedores para la fabricación de dispositivos optoelectrónicos y otras aplicaciones, por lo que el control de su morfología mediante métodos químicos es de gran importancia.

Nanofibras de AlN

El nitruro de aluminio (AlN) es un material muy importante para la debido a su alta conductividad térmica, bajo coeficiente de expansión térmica y alto stress mecánico. Además, AlN es un aislador con un band gap de energía amplio, alta resistividad y baja constante dieléctrica. Los nanocables y nanofibras de AlN han atraído mucha atención debido a que se supone que sus estructuras cuasi perfectas poseen mayor conductividad térmica que el AlN policristalino y podrían proveer mejor refuerzo a sus componentes ${ }^{18}$.

Como se ha visto con anterioridad, la mayoría de los métodos utilizados para la síntesis y obtención de nitruros IIIA requieren de equipamiento costoso y de alto nivel, temperaturas elevadas y precursores químicos tóxicos, lo que hace que existan amplios esfuerzos actualmente para encontrar nuevas rutas para la obtención de los nitruros de alta pureza. En particular, el mayor problema de sintetizar nanofibras de AlN de alta pureza a bajas temperaturas es cómo controlar la morfología y fase de los productos. Wu y colaboradores ${ }^{19}$ prepararon $\mathrm{AlN}$ hexagonal con morfología de fibras usando $\mathrm{AlC}_{3}$ como fuente de aluminio y $\mathrm{NaN}_{3}$ como fuente de nitrógeno en un sistema libre de solvente a una temperatura de $450^{\circ} \mathrm{C}$, proceso que se explica mediante la siguiente reacción:

$\mathrm{AlCl}_{3}+3 \mathrm{NaN}_{3} \rightarrow \mathrm{AlN}+3 \mathrm{NaCl}+4 \mathrm{~N}_{2}$ 
Los productos obtenidos son nanofibras de fase hexagonal (Figura 1.3), con un ancho de 10 a 80 nm.

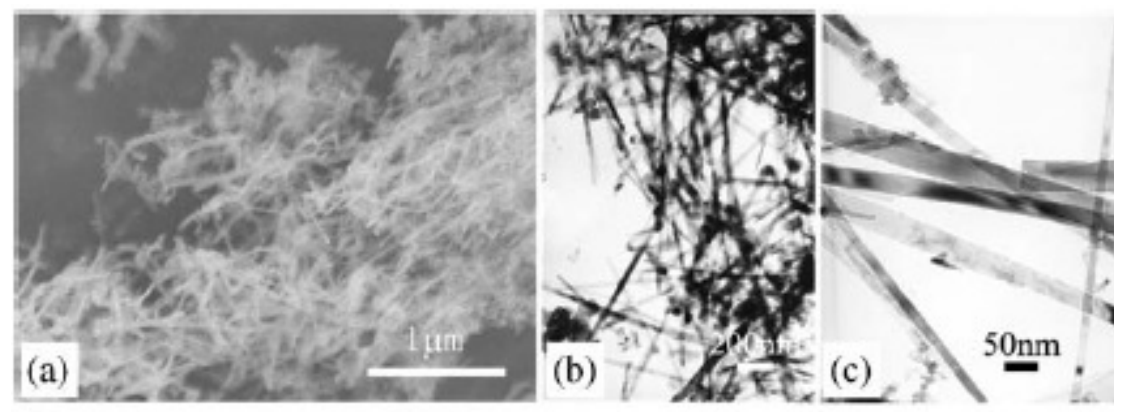

Figura 1.3. Imágenes de microscopía electrónica de las nanofibras de AlN. (a) imagen típica, (b) y (c) imágenes magnificadas.

La temperatura jugó un papel importante, no pudiéndose obtener nanofibras con una temperatura menor a $300{ }^{\circ} \mathrm{C}$ durante la reacción. Por otro lado, a temperaturas mayores a $550{ }^{\circ} \mathrm{C}$ los autores obtuvieron partículas en vez de fibras de AlN. Asimismo, cuando $\mathrm{AlCl}_{3}$ fue sustituido por aluminio comercial, no se obtuvieron fibras de AlN, aun incrementando la temperatura hasta 650 ${ }^{\circ} \mathrm{C}$. Tampoco se obtuvo morfología de fibras en el material si se utilizaba $\mathrm{NaNH}_{2}, \mathrm{Mg}_{3} \mathrm{~N}_{2} \mathrm{O}$ $\mathrm{NH}_{4} \mathrm{Cl}$ como fuente de nitrógeno.

\section{Nanoestructuras de $B N$}

El BN es un análogo estructural del carbono, existiendo en forma cúbica (tipo diamante), hexagonal (tipo grafito) y de forma amorfa, exhibiendo uniones B-N en vez de C-C. La longitud de enlace, parámetros de orden de largo alcance y las constantes de red son muy similares entre $\mathrm{BN}$ hexagonal y grafito. Basado en estas similitudes, es de esperar que BN forme estructuras de tipo fullereno o nanotubos, los cuales han sido producidos por una variedad de métodos ${ }^{20}$. Ha sido reportado que las esferas huecas con cavidades mayores pueden exhibir una estructura más defectuosa que las estructuras de tipo fullereno-cebolla, lo que las hace más útiles para aplicaciones potenciales en adsorción de hidrógeno. Sin embargo, no existe un método definido en la actualidad para la obtención de esferas huecas de BN en cantidades significativas. Xu y coautores $^{21}$ reportaron una pequeña proporción de esferas huecas de $\mathrm{BN}$ en cohexistencia con nanotubos en la co-pirólisis de $\mathrm{NH}_{4} \mathrm{BF}_{4}, \mathrm{KBH}_{4} \mathrm{Y} \mathrm{NaN}$, en el rango de $450-600{ }^{\circ} \mathrm{C}$. Wang y colaboradores $^{22}$ reportaron una ruta para preparar esferas huecas de $\mathrm{BN}$ con un rendimiento del $30-40 \%$, mediante la siguiente reacción:

$\mathrm{BBr}_{3}+3 \mathrm{NaN}_{3} \rightarrow \mathrm{BN}+3 \mathrm{NaBr}+4 \mathrm{~N}_{2}$

Los autores postulan que la producción de nitrógeno gaseoso en el proceso aumenta la probabilidad de formación de las esferas huecas de BN.

\section{Microspindles de GaN}

La dimensionalidad del material juega un papel importante en las propiedades físicas de los materiales, por lo que uno de los objetivos de la síntesis es intentar controlar la forma de las nanoestructuras en el nivel mesoscópico. Las estructuras unidimensionales (1D) de GaN, por ejemplo, son candidatas atractivas para montar dispositivos activos a nanoescala para 
aplicaciones electrónicas y ópticas. Sin embargo, como se expuso en casos anteriores, los métodos de síntesis requieren condiciones de reacción críticas y eso limita sus aplicaciones a gran escala. Asimismo, como la aplicación a gran escala de semiconductores es sensible a la forma que éstos tengan y a cambios en su morfología, es necesaria una exploración profunda de la síntesis y el crecimiento de estructuras de GaN y de la influencia de condiciones externas en la morfología de las mismas.

$\mathrm{Xu}$ y coautores ${ }^{23}$ reportaron una síntesis a gran escala de microspindles de GaN por medio de una reacción de estado sólido en la cual los materiales de partida $\mathrm{GaI}_{3}, \mathrm{NaNH}_{2}$ y $\mathrm{NH}_{4} \mathrm{Cl}$ se mantuvieron a $500{ }^{\circ} \mathrm{C}$ por 6 horas en un sistema sellado.

Nanocables de GaP y InP

En la última década, se ha realizado un progreso significativo en el crecimiento de nanocables de los materiales inorgánicos más importantes con el objeto de mejorar la uniformidad y aumentar el rendimiento del material obtenido, por lo que los nanocables han sido desarrollados por varios métodos. Generalmente, los nanocables tienen síntesis y mecanismos de crecimiento únicos, dependiendo del material y del enfoque con que se aborde el problema (físico o químico).

Los semiconductores IIIA de fósforo unidimensionales son materiales prometedores para la fabricación de nanodispositivos optoelectrónicos. Recientemente, han habido esfuerzos para explorar nuevas rutas para sintetizar nanocables de estos materiales. Desde el punto de vista de la química verde, el agua es un medio ideal para la ruta de obtención de nanocables IIIA de fósforo, llevando al grupo de Xiong y coautores ${ }^{24}$ a investigar la obtención de estas nanoestructuras en solución acuosa y bajo condiciones menos críticas. Estos autores llevaron a cabo una estrategia general, adaptada del método de Li y colaboradores ${ }^{25}$, donde las estructuras de nanocables pueden ser formadas en el borde de estructuras laminares las cuales, a su vez, pueden ser obtenidas por medio de mecanismos de co-condensación en la presencia y composición adecuada de surfactante, agua, aceite y co-surfactante. La reacción utilizada es la que sigue:

$[\mathrm{CTA}]^{+}+\left[\mathrm{M}(\mathrm{OH})_{4}\right]^{-} \rightarrow[\mathrm{CTA}]^{+}\left[\mathrm{M}(\mathrm{OH})_{4}\right]^{-}$

$\mathrm{P}_{4}+3 \mathrm{OH}^{-}+3 \mathrm{H}_{2} \mathrm{O} \rightarrow \mathrm{PH}_{3}+3 \mathrm{H}_{2} \mathrm{PO}_{2}^{-}$

$[\mathrm{CTA}]^{+}\left[\mathrm{M}(\mathrm{OH})_{4}\right]^{-}+\mathrm{PH}_{3} \rightarrow \mathrm{MP}+3 \mathrm{H}_{2} \mathrm{O}+\mathrm{OH}^{-}+\mathrm{CTA}^{+}$

Donde CTA es cetiltrimetilamonio y $\left[\mathrm{M}(\mathrm{OH})_{4}\right]^{-}$corresponde al hidróxido de Ga o In. Las estructuras laminares de surfactante-material inorgánico $[\mathrm{CTA}]^{+}\left[\mathrm{M}(\mathrm{OH})_{4}\right]^{-}$fueron observadas por difracción de rayos $\mathrm{X}$.

Se espera que esta estrategia sea generalizada para el crecimiento de nanoestructuras de otros materiales en solución acuosa, dado que se puede realizar seleccionando la reacción apropiada y sus correspondientes precursores laminares.

\subsection{Reactividad química de nanocristales de GaP en síntesis orgánica}

Es sabido que las nanopartículas pueden proveer oportunidades potenciales para la síntesis de compuestos orgánicos. Gao y coautores ${ }^{26}$ investigaron la posibilidad del GaP nanocristalino de inducir reordenamientos térmicos en el benceno en una reacción orgánica, y realizar exitosamente 
una conversión de alto rendimiento del benceno a 6-fenilfulveno bajo condiciones supercríticas, siendo de gran interés, dado que el reordenamiento térmico del benceno ha sido muy difícil de lograr en otras oportunidades. GaP favorece el reordenamiento del benceno, apoyando la suposición de que las nanopartículas inorgánicas favorecerían algunas reacciones orgánicas y abre una nueva puerta a las aplicaciones.

El proceso completo cuyo mecanismo no es conocido, se muestra en la Figura 1.4. El mismo podría producirse en tres pasos: (1) las moléculas de benceno se adsorben químicamente en la superficie de los nanocristales de GaP y experimentan una dimerización exitosa a una temperatura de $450-500{ }^{\circ} \mathrm{C}$ para formar el compuesto I; (2) el compuesto I se transforma en II, lo que los autores confirmaron mediante el cálculo teórico de su entalpía; y (3) basado en otro cálculo con herramientas de química computacional, se postula que el compuesto II se transforma en el producto final, 6-fenilfulveno, mediante la eliminación de dos átomos de hidrógeno. Debido a la mayor superficie y a una mayor actividad catalítica de los cristales de GaP, la reacción ocurriría en un tiempo relativamente rápido. No se observó la misma capacidad en nanocristales de GaAs, InP y CdS.

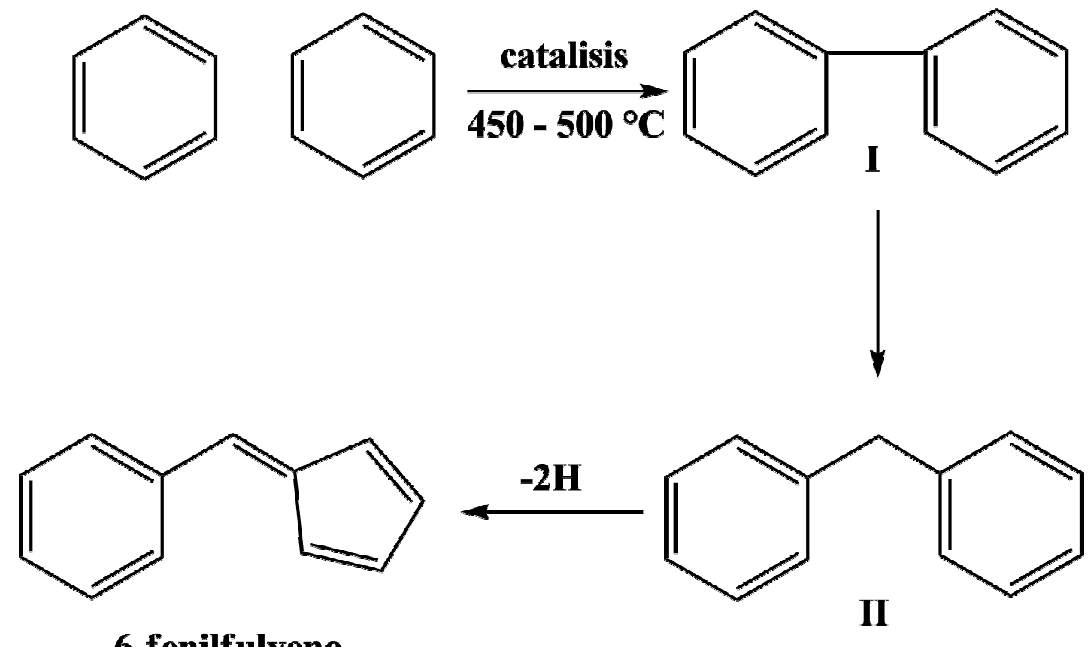

Figura 1.4. Esquema de rearreglo térmico y dimerización de benceno catalizado por nanocristales de GaP.

\subsection{Estabilidad térmica de GaP nanocristalino}

\section{Estabilidad de GaP en solvente y temperatura}

Otro de los estudios que Gao y coautore ${ }^{27}$ realizaron involucró el proceso de crecimiento, la estabilidad de nanocristales de $\mathrm{GaP}$ y la formación de nanocristales $\mathrm{Ga}_{3} \mathrm{P}$ en solvente y bajo temperatura. GaP se formó por la reacción:

$\mathrm{Na}_{3} \mathrm{P}+\mathrm{GaCl}_{3} \leftrightarrow \mathrm{GaP}+3 \mathrm{NaCl}$

Pero al prolongar el tiempo de reacción, se produjo $\mathrm{Ga}_{3} \mathrm{P}$. Los autores postularon que bajo condiciones térmicas $\left(300-400{ }^{\circ} \mathrm{C}\right)$ y con benceno como solvente, la descomposición de GaP 
debe tener lugar cuando el tiempo de reacción se prolonga o la temperatura se incrementa, obedeciendo a la siguiente reacción:

$6 \mathrm{GaP} \leftrightarrow 2 \mathrm{Ga}_{3} \mathrm{P}+\mathrm{P}_{4}$

Estabilidad térmica de GaP en oxígeno

En resumen, GaP nanocristalino no es estable en $\mathrm{O}_{2}$ a temperatura elevada ${ }^{28}$ y puede ser fácilmente oxidable a una temperatura un poco menor que $400{ }^{\circ} \mathrm{C}$, mucho más baja que la temperatura a la que se oxida el material masivo dado que la gran área de superficie del GaP nanocristalino puede reducir marcadamente la dificultad de oxidación. Esta capacidad puede ser utilizada para proveer una base confiable para la aplicación de nanocristales de GaP bajo condiciones ambientales. Asimismo, puede ser utilizada para sintetizar diferentes morfologías de $\mathrm{Ga}_{2} \mathrm{O}_{3}$ nanocristalino mediante el control de los rangos de calentamiento.

Estabilidad térmica de nanocristales de GaP en nitrógeno

Una nueva y conveniente técnica para formar nanopartículas y nanocables de $\mathrm{GaN}$ fue establecida $^{29}$ con base en que el pequeño tamaño del GaP precursor puede disminuir significativamente la dificultad de incorporar el $\mathrm{N}_{2}$ y formar GaN. Como resultado, nanopartículas y nanocables de $\mathrm{GaN}$ cúbico fueron sintetizados con éxito a partir de $\mathrm{GaP}_{\text {y }} \mathrm{N}_{2}$ a $500{ }^{\circ} \mathrm{C}$. La estabilidad térmica de $\mathrm{GaP}$ nanocristalino bajo $\mathrm{N}_{2}$ y a diferentes rangos de temperatura respalda la propuesta de que GaN cúbico puede formarse vía un mecanismo de desplazamiento N-P.

Se observa que GaN se forma gradualmente en la superficie de las nanopartículas de GaP. La gran área superficial y reactividad del GaP resulta directamente en la formación de GaN a menor temperatura, menor presión y tiempos de reacción más cortos. Las nanopartículas GaN resultantes heredan las matrices de $\mathrm{GaP}$ en términos de estructura y morfología, encontrándose múltiples similitudes entre GaP y GaN.

\subsection{Comentarios finales}

Se expuso la obtención de nanocompuestos constituidos por elementos de los grupos III y V. Debido a la necesidad de condiciones muy extremas (temperaturas altas y reactivos tóxicos y peligrosos) y equipamiento especializado para la preparación de estas nanoestructuras, las herramientas de química computacional aplicadas al estudio de estos compuestos pueden ayudar a comprender y conocer su formación y propiedades. Asimismo, aunque los estudios teóricos se centren en el estudio de agregados pequeños, éstos también constituyen sistemas de interés por sí mismos, debido a sus propiedades y comportamiento excepcionales.

En este trabajo estudiamos el crecimiento de agregados pequeños III/V, de tipo $\mathrm{X}_{\mathrm{n}} \mathrm{Y}_{\mathrm{m}}, \mathrm{X}=\mathrm{B}, \mathrm{Al}$; $\mathrm{Y}=\mathrm{N}, \mathrm{P} ; \mathrm{n}=1-4$ y $\mathrm{m}=1-4(\mathrm{n}+\mathrm{m} \leq 5)$. El trabajo está organizado de la siguiente manera: en el primer capítulo se describen los métodos de la química computacional utilizados en conjunto con su formulación matemática; en los capítulos siguientes, 2 a 5, se detallan los resultados obtenidos para agregados de $\mathrm{B} / \mathrm{N}, \mathrm{Al} / \mathrm{N}, \mathrm{B} / \mathrm{P}$ y $\mathrm{Al} / \mathrm{P}$, respectivamente; luego, en la sección 6 , se presentan colcusiones generales de los resultados del crecimiento de los agregados III/V estudiados. Finalmente, se presentan tres apéndices, siendo el primero la metodología de análisis preliminares realizados a los sistemas $\mathrm{Al} / \mathrm{N}$ y $\mathrm{B} / \mathrm{N}$; en el segundo se muestran los resultados de 
los análisis preliminares de $\mathrm{Al} / \mathrm{N}$ y en el tercero los resultados de los análisis preliminares de $\mathrm{B} / \mathrm{N}$. 


\section{Metodología}

Si estamos interesados en describir la distribución electrónica de un sistema químico en detalle, no hay sustituto para la mecánica cuántica. Los electrones son partículas muy livianas y no pueden ser descriptos correctamente aún cualitativamente por la mecánica clásica. Nos concentraremos en la ecuación de Schrödinger independiente del tiempo:

\section{$H \Psi=E \Psi$}

Un aporte esencial para resolver la ecuación de Schrödinger es la aproximación de BornOppenheimer, donde se desprecia el acoplamiento entre el movimiento electrónico y el nuclear. Esto permite que la parte electrónica se resuelva con las posiciones nucleares como parámetros, y la resultante superficie de energía potencial (PES, por sus siglas en inglés, potential energy surface), constituye la base para resolver el movimiento nuclear. El mayor esfuerzo computacional está en resolver la ecuación de Schrödinger para un conjunto dado de coordenadas nucleares.

La dinámica de un sistema polielectrónico es muy compleja $\mathrm{y}$, consecuentemente, requiere de métodos computacionales elaborados. Una simplificación significativa computacional y conceptualmente hablando, puede hacerse introduciendo modelos de partículas independientes, donde el movimiento de un electrón es considerado independiente de la dinámica de todos los otros electrones. En un modelo de partícula independiente las interacciones entre las partículas son aproximadas, ya sea despreciando todas menos la más importante, o teniendo en cuenta todas las interacciones de manera promediada. Dentro de la teoría de estructura electrónica, sólo la última opción tiene una exactitud aceptable, y se le llama teoría de Hartree-Fock (HF). En el modelo de HF, se describe cada electrón por un orbital, y la función de onda total está dada por un producto de orbitales. Como los electrones son fermiones, la función de onda debe ser antisimétrica (debe cambiar el signo al intercambiar las coordenadas de dos electrones), lo que se logra convenientemente arreglando los orbitales en un determinante de Slater. El mejor conjunto de orbitales se determina por el principio variacional; esto es, los orbitales HF dan la energía más baja, dentro de la restricción de que la función de onda esté descripta por un determinante de Slater. La forma de un orbital atómico o molecular dado describe la probabilidad de encontrar un electrón, donde la atracción a el o los núcleos y la repulsión promedio a los otros electrones están incluidas. Como los otros electrones están descriptos, a su vez, por sus respectivos orbitales, las ecuaciones de HF dependen de sus propias soluciones, y deben ser resueltas iterativamente. Cuando los orbitales son expandidos en un conjunto de funciones conocidas, las ecuaciones resultantes pueden ser escritas como un problema matricial de autovalores. El modelo de HF es un punto medio, donde se pueden hacer aproximaciones adicionales, llevando a métodos semiempíricos; o puede ser mejorada agregando determinantes adicionales, generando modelos que pueden converger a la solución exacta de la ecuación de Schrödinger, dependiendo del conjunto de funciones utilizado para representar los orbitales.

Los métodos semiempíricos se derivan del modelo HF, despreciando todas las integrales que involucran más de dos núcleos en la construcción de la matriz de Fock. El éxito de éste tipo de métodos yace en convertir las integrales remanentes en parámetros, y ajustarlos a datos experimentales o calculados con métodos más sofisticados, especialmente energías moleculares y geometrías. Dichos métodos son computacionalmente mucho menos costosos que el método $a b$ 
initio HF, pero están limitados a sistemas donde los parámetros existen; además, teniendo en cuenta que ya la aproximación de HF tiene exactitud limitada, las aproximaciones que estos métodos realizan llevarán a modelos más pobres.

La teoría HF sólo tiene en cuenta interacciones electrón-electrón promediadas, y consecuentemente desprecia la correlación entre electrones. Los métodos que incluyen la correlación electrónica requieren una función de onda multideterminantal, dado que HF describe la mejor función de onda de un solo determinante. Los métodos multideterminantales son mucho más costosos computacionalmente, pero pueden llevar a resultados que sistemáticamente se acercan a la solución exacta de la ecuación de Schrödinger. Estos métodos son llamados métodos de correlación electrónica, ellos son: configuración de interacciones, teoría de perturbaciones y métodos de coupled cluster, y no serán tratados en este trabajo.

La teoría del funcional de la densidad (DFT, density functional theory) es una alternativa interesante a la teoría HF, donde el efecto de la correlación electrónica se modela con una función de la densidad electrónica. La DFT provee significativamente mejores resultados que HF. A continuación, se describe esta teoría, que se utilizó como metodología de cálculo para los sistemas de estudio, con algunos comentarios respecto a sus diferencias con HF.

\subsection{Teoría del funcional de la densidad}

La base de la teoría del funcional de la densidad es la prueba, por Hohenberg y $\mathrm{Kohn}^{30}$, de que la energía electrónica del estado fundamental de un sistema de $\mathrm{N}$ electrones está determinada completamente por la densidad electrónica $\rho$. En otras palabras, existe una correspondencia entre la densidad electrónica de un sistema y su energía. El significado de esto, tal vez, se verá mejor si comparamos con el enfoque de la función de onda. Una función de onda de un sistema $N$-electrónico contiene $3 N$ coordenadas, tres para cada electrón (cuatro si se incluye al espín). La densidad electrónica es el cuadrado de la función de onda, integrada sobre N-1 coordenadas electrónicas, ésta solo depende de tres coordenadas, independientemente del número de electrones. Mientras que la complejidad de la función de onda aumenta con el número de electrones, la densidad electrónica tiene el mismo número de variables, independientemente del tamaño del sistema. El problema es que, a pesar de que ha sido probado que cada densidad diferente da una energía fundamental diferente, no se conoce el funcional que conecta a estas dos cantidades. Por lo tanto, el objetivo más importante de la teoría del funcional de la densidad es diseñar funcionales que conecten a la densidad electrónica con la energía del estado fundamental ${ }^{31}$.

Se debe tener en cuenta el significado de funcional. Una función $f(x)$ es una receta para producir un número a partir de un conjunto de variables; mientras que un funcional $F[f]$ es una receta similar, pero para producir un número a partir de una función. La función de onda y la densidad son funciones, mientras que una energía que depende de la función de onda o de una densidad electrónica, es un funcional.

La densidad electrónica es un observable físico que, integrada sobre todo el espacio da el número total de electrones en el sistema ${ }^{32}, \mathrm{~N}$,

$$
N=\int \rho(r) d r
$$


Como los núcleos se toman como cargas puntuales, sus posiciones corresponden a máximos locales en la densidad electrónica, entonces sólo falta asignar números atómicos nucleares para especificar completamente el Hamiltoniano. Se puede demostrar que ésta información también está disponible a partir de la densidad, dado que para cada núcleo A localizado en un máximo de la densidad electrónica, $\mathrm{r}_{\mathrm{A}}$

$$
\left.\frac{\partial \rho\left(r_{A}\right)}{\partial r_{A}}\right|_{r_{A}=0}=-2 Z_{A} \rho\left(r_{A}\right)
$$

donde $\mathrm{Z}_{\mathrm{A}}$ es el número atómico de $\mathrm{A}, \mathrm{r}_{\mathrm{A}}$ es la distancia radial desde $\mathrm{A}, \mathrm{y} \rho$ es la densidad promediada esféricamente. En la ecuación anterior y en adelante se utilizarán unidades atómicas. Por lo tanto, lo que éstas expresiones quieren indicar es que dada una densidad conocida, se puede formar el operador Hamiltoniano, resolver la ecuación de Schrödinger y determinar las funciones de onda y los autovalores, las energías.

\section{Primeras aproximaciones}

La energía se puede separar en componentes cinéticos y potenciales. Si se decide evaluar la energía de un sistema usando sólo su densidad electrónica como variable, el enfoque más simple es considerar el sistema como clásico, en cuyo caso los componentes de la energía potencial son determinados de manera muy directa. La atracción entre los núcleos y la densidad es,

$$
V_{n e}[\rho(r)]=-\sum_{k}^{\text {núcleos }} \int \frac{Z_{k}}{\left|r-r_{k}\right|} \rho(r) d r
$$

y la repulsión de una distribución de carga clásica consigo misma es,

$V_{e e}[\rho(r)]=\frac{1}{2} \iint \frac{\rho\left(r_{1}\right) \rho\left(r_{2}\right)}{\left|r_{1}-r_{2}\right|} d r_{1} d r_{2}$

donde $\mathrm{r}_{1} \mathrm{y}_{2}$ son variables ficticias de integración actuando sobre todo el espacio.

La energía cinética de una distribución de carga continua es menos obvia. Para proceder, introduciremos una sustancia ficticia, un sistema compuesto por un número infinito de electrones moviéndose en un volumen infinito de espacio que se caracteriza por tener una carga positiva uniformemente distribuída. Esta distribución electrónica se llama comúnmente gas electrónico uniforme y tiene una densidad constante distinta de cero. Thomas ${ }^{33}$ y Fermi ${ }^{34}$, en 1927, usaron la mecánica estadística de fermiones para derivar la energía cinética de este sistema de la siguiente manera:

$$
T_{\text {geu }}[\rho(r)]=\frac{3}{10}\left(3 \pi^{2}\right)^{2 / 3} \int \rho^{5 / 3}(r) d r
$$


Es importante notar que los términos descriptos en las expresiones (2.3) a (2.5) son funciones de la densidad, que a su vez es función de coordenadas espaciales tridimensionales. Como se había mencionado con anterioridad, una función cuyo argumento es una función, se llama funcional, y entonces los términos $\mathrm{T}$ y $\mathrm{V}$ son funcionales de la densidad. Las ecuaciones de Thomas-Fermi ((2.3) a (2.5)) representan el primer esfuerzo para definir una teoría del funcional de la densidad (DFT), donde la energía es calculada sin ninguna referencia a la función de onda. Sin embargo, mientras estas ecuaciones son importantes para la historia de la química, las suposiciones subyacentes son lo suficientemente inexactas como para no encontrar uso en la química moderna (en la DFT de Thomas-Fermi todas las moléculas son inestables en relación con la disociación en sus átomos constituyentes).

Una gran aproximación es el uso de la ecuación (2.4) para la repulsión interelectrónica, dado que ignora los efectos energéticos asociados con el intercambio y la correlación. Es útil introducir el concepto de función cavidad, que se define de manera que corrija los errores energéticos por la asunción de comportamiento clásico. En particular,

$$
\left\langle\Psi\left|\sum_{i<j}^{\text {electrones }} \frac{1}{r_{i j}}\right| \Psi\right\rangle=\frac{1}{2} \iint \frac{\rho\left(r_{1}\right) \rho\left(r_{2}\right)}{\left|r_{1}-r_{2}\right|} d r_{1} d r_{2}+\frac{1}{2} \iint \frac{\rho\left(r_{1}\right) h_{x c}\left(r_{1} ; r_{2}\right)}{\left|r_{1}-r_{2}\right|} d r_{1} d r_{2}
$$

la primera integral en la ecuación (2.6) es la repulsión electrónica clásica. El segundo término corrige las deficiencias del primer término usando la función cavidad, $h_{x c}$ asociada con $\rho$. La notación $h_{x c}\left(r_{1} ; r_{2}\right)$ indica que la cavidad o hueco está centrada en la posición del electrón 1 , y es evaluado desde allí como una función de las coordenadas espaciales que definen $r_{2}$; observemos, entonces, que no sólo el valor de $h$ varía como una función de $r_{2}$ para un dado valor de $r_{1}$, sino que la forma precisa de $h$ puede variar como función de $r_{l}$.

El hueco de intercambio y correlación, como es usualmente llamada la función cavidad, se define de la siguiente manera:

$h_{x c}\left(r_{1}, r_{2}\right)=\frac{\rho_{2}\left(r_{1}, r_{2}\right)}{\rho_{1}\left(r_{1}\right)}-\rho_{1}\left(r_{2}\right)$

éste representa la probabilidad de encontrar al electrón 2 en posición $r_{2}$, siendo que el electrón 1 está en $r_{l}$. La parte de intercambio de $h_{x c}$ se llama hueco de Fermi, mientras que la de correlación se llama hueco de Coulomb. Dado que el intercambio sólo ocurre entre electrones del mismo espín, el hueco total puede ser escrito también en términos de las contribuciones de espín individuales:

$h_{x c}=h_{x}+h_{c}$

$h_{x}=h_{x}^{\alpha \alpha}+h_{x}^{\beta \beta}$

$h_{c}=h_{c}^{\alpha \alpha}+h_{c}^{\beta \beta}+h_{c}^{\alpha \beta}$

Por su construcción, la teoría de Hartree-Fock (HF) evita cualquier error por autointeracción de un electrón consigo mismo y evalúa exactamente la energía de intercambio; sin embargo, consume mucho tiempo evaluar las integrales dielectrónicas multicéntricas a partir de las cuales estas energías son calculadas. Slater ${ }^{35}$ observó que una consecuencia del principio de exclusión de Pauli es que el hueco de intercambio de Fermi (ecuaciones (2.7) y (2.8)), es más grande que el 
hueco de correlación, es decir, las correcciones a la repulsión clásica debidas al intercambio son significativamente mayores que las correcciones de correlación. Propuso ignorar la correlación y sugirió que el hueco de intercambio alrededor de cualquier posición podía ser aproximado como una esfera de potencial constante con un radio dependiente de la magnitud de la densidad en esa posición. Dentro de esta aproximación, la energía de intercambio $E_{x}$ se determina de la siguiente manera:

$E_{x}[\rho(r)]=-\frac{9 \alpha}{8}\left(\frac{3}{\pi}\right)^{1 / 3} \int \rho^{4 / 3}(r) d r$

Dentro de la derivación de Slater, el valor de la constante $\alpha$ es 1 y la ecuación (2.9) define el llamado funcional de la energía de intercambio de Slater.

Empezando a partir del gas electrónico uniforme, Bloch y Dirac habían derivado una expresión similar varios años antes, excepto que en ese caso $\alpha=\frac{2}{3}$. La combinación de esta expresión con las ecuación (2.3) a (2.5) define el modelo de Thomas-Fermi-Dirac, aunque también es bastante inexacto y no se usa en la actualidad.

Dados los diferentes valores de $\alpha$ en la ecuación (2.9) como función de varias derivaciones diferentes, varios científicos consideraron oportuno tratarlo como un valor empírico, y los cálculos que emplean la ecuación (2.9) se llaman cálculos X $\alpha$. Análisis empíricos en una variedad de sistemas diferentes sugieren que $\alpha=\frac{3}{4}$ provee mejores resultados y más exactos que $\alpha=1 \mathrm{o}$ $\alpha=\frac{2}{3}$. Esta metodología particular de DFT no es comúnmente utilizada.

\section{Fundamentación}

El trabajo descripto en la sección anterior fue provocativo en su tiempo en comparación a los enfoques basados en la función de onda, debido a su simplicidad. Como resultado de ello, los modelos más tempranos de DFT encontraron uso extendido en la comunidad de físicos que estudiaba el estado sólido, donde el modelo del gas electrónico uniforme describe con éxito la estructura de sólidos metálicos e iónicos, no así la estructura de moléculas. Este estado de las cosas cambió cuando Hohenberg y $\mathrm{Kohn}^{30}$ probaron dos teoremas críticos para establecer a la DFT como una metodología químico cuántica legítima. Cada uno de estos teoremas será presentado a continuación de manera abreviada.

\section{El primer teorema de Hohenberg-Kohn}

En el lenguaje de DFT, los electrones interactúan uno con el otro y con un potencial externo. Entonces, en el modelo del gas uniforme, el potencial externo es la carga positiva distribuida uniformemente, y en un átomo o una molécula, el potencial externo es la atracción al o los núcleos dada por la expresión usual mostrada en la ecuación (2.3). Como se señaló anteriormente, para establecer una dependencia de la energía con la densidad del estado fundamental, es 
suficiente con demostrar que esta densidad determina el operador Hamiltoniano. Asimismo, la integración sobre la densidad nos da el número de electrones, entonces lo único que queda para definir el operador es la determinación del potencial externo (esto es, las cargas y posiciones de los núcleos).

Si hubiera dos potenciales externos $v$ y $v^{\prime}$, que difieren por más de una constante, cada uno teniendo la misma $\rho$ para su estado fundamental, tendríamos dos Hamiltonianos, $H$ y $H^{\prime}$ cuyas densidades de estado fundamental serían las mismas, aunque las funciones de onda normalizadas $\Psi$ y $\Psi^{\prime}$ serían diferentes. Tomando $\Psi^{\prime}$ como función de prueba para $H$, tendríamos (usando $E[\Psi] \geq E_{0}$ por el principio variacional ${ }^{36}$ ):

$$
\begin{aligned}
E_{0}<\left\langle\Psi^{\prime}|H| \Psi^{\prime}\right\rangle & =\left\langle\Psi^{\prime}\left|H^{\prime}\right| \Psi^{\prime}\right\rangle+\left\langle\Psi^{\prime}\left|H-H^{\prime}\right| \Psi^{\prime}\right\rangle \\
& =E_{0}^{\prime}+\int \rho(r)\left[v(r)-v^{\prime}(r)\right] d r
\end{aligned}
$$

donde $E_{0}$ y $E_{0}^{\prime}$ son las energías de estado fundamental para $H$ y $H^{\prime}$, respectivamente. Similarmente, tomando $\Psi$ como función de prueba para $H^{\prime}$ :

$$
\begin{aligned}
E_{0}^{\prime}<\left\langle\Psi\left|H^{\prime}\right| \Psi\right\rangle & =\langle\Psi|H| \Psi\rangle+\left\langle\Psi\left|H^{\prime}-H\right| \Psi\right\rangle \\
& =E_{0}-\int \rho(r)\left[v(r)-v^{\prime}(r)\right] d r
\end{aligned}
$$

Sumando (2.10) y (2.11), obtendríamos $E_{0}+E_{0}^{\prime}<E_{0}^{\prime}+E_{0}$, que es imposible. Entonces, no puede haber dos $v$ diferentes que den la misma $\rho$ para sus estados fundamentales.

$\rho$ determina $N$ y $v$, y por lo tanto, todas las propiedades del estado fundamental, como la energía cinética $T[\rho]$, la energía potencial $V[\rho]$ y la energía total $E[\rho]$. Escribiendo $E_{v}$ por $E$, para hacer explícita la dependencia en $v$,

$E_{v}[\rho]=T[\rho]+V_{n e}[\rho]+V_{e e}[\rho]=\int \rho(r) v(r) d r+F_{H K}[\rho]$

donde $F_{H K}[\rho]=T[\rho]+V_{e e}[\rho]$ y $V_{n e}[\rho]$ se conoce, pero los funcionales $T[\rho]$ y $V_{e e}[\rho]$ son desconocidos. Notemos que $F_{H K}[\rho]$ en la ecuación anterior se define independientemente del potencial externo $v(r)$, esto significa que $F_{H K}[\rho]$ es un funcional universal de $\rho(r)$. Una vez que tenemos una forma explícita de $F_{H K}[\rho]$, podemos aplicar este método a cualquier sistema. Pero la ecuación (2.12) no proporciona una vía práctica para calcular la energía a partir de la densidad electrónica, debido al desconocimiento del funcional $F_{H K}[\rho]$. Para transformar esta ecuación en una herramienta práctica, Hohenberg y Kohn desarrollaron un segundo teorema.

\section{EI teorema variacional de Hohenberg-Kohn}

El primer teorema de Hohenberg y Kohn es un teorema de existencia. Como tal, introduce el potencial externo, pero es inútil en proveer un indicio de cómo predecir la densidad del sistema. 
Se necesita un mecanismo para obtener nuestra cantidad fundamental. Hohenberg y Kohn ${ }^{30}$ mostraron en un segundo teorema que la energía obedece un principio variacional.

Primero, supongamos que se tiene una densidad de prueba que satisface las condiciones de contorno e integra al número de electrones, $N$. En ese caso, el primer teorema indica que esta densidad determina una función de onda de prueba $\widetilde{\Psi}$ y un Hamiltoniano. Planteado el caso, se pueden evaluar los valores esperados de la energía:

$$
\langle\widetilde{\Psi}|H| \Psi\rangle=\widetilde{E} \geq E_{0}
$$

la cual, por el principio variacional ${ }^{37}$, debe ser mayor o igual a la verdadera energía del estado fundamental. Entonces, en principio, se pueden seguir eligiendo diferentes densidades y aquellas que proveen menores energías, calculando con (2.13), están más cerca de la correcta. Dicho procedimiento es, por supuesto, insatisfactorio.

La dificultad yace en la naturaleza del funcional $F_{H K}[\rho]$. Hasta este punto, se ha indicado qué hay y cómo se calcula, pero no se ha sugerido ningún mecanismo por el cual la densidad puede ser utilizada como argumento en alguna ecuación variacional general característica. Dicho enfoque apareció por primera vez en 1965.

\subsection{Método de Kohn-Sham}

Si conocemos la densidad electrónica del estado fundamental $\rho_{0}(r)$, el teorema de Hohenberg y Kohn (HK) nos dice que es posible, en principio, calcular todas las propiedades moleculares del estado fundamental a partir de $\rho_{0}$ sin haber tenido que obtener la función de onda. El problema es que el teorema de HK no nos dice cómo calcular $E_{0}$ a partir de $\rho_{0}$, ya que el funcional $F_{H K}[\rho]$ en (2.12) es desconocido; ni nos dice cómo obtener $\rho_{0}$ sin obtener primeramente la función de onda. Un paso clave hacia estos objetivos lo dieron Kohn y Sham en $1965^{37}$, quienes obtuvieron un método práctico para obtener $E_{0}$ a partir de $\rho_{0}$.

Kohn y Sham consideraron un sistema de referencia ficticio (denotado mediante el subíndice s), de $N$ electrones no interactuantes que experimentan todos ellos la misma función de energía potencial, representada por $v_{s}\left(r_{i}\right)$, que hace que la densidad de probabilidad electrónica del estado fundamental de referencia $\rho_{s}(r)$, sea igual a densidad electrónica del estado fundamental exacta $\rho_{0}(r)$, del sistema de interés: $\rho_{s}(r)=\rho_{0}(r)$. Ya que Hohenberg y Kohn demostraron que la función de densidad de probabilidad del estado fundamental determina el potencial externo, una vez que $\rho_{s}\left(r_{i}\right)$ está definido por el sistema de referencia, el potencial externo $v_{s}\left(r_{i}\right)$ en el sistema de referencia está unívocamente determinado. Los electrones no interactúan entre sí en el sistema de referencia, de forma que el Hamiltoniano del sistema de referencia es,

$$
H_{s}=\sum_{i=1}^{n}\left[-\frac{1}{2} \nabla_{i}^{2}+v_{s}\left(r_{i}\right)\right] \equiv \sum_{i=1}^{n} h_{i}^{K S} \quad \text { donde } \quad h_{i}^{K S} \equiv-\frac{1}{2} \nabla_{i}^{2}+v_{s}\left(r_{i}\right)
$$


$h_{i}^{K S}$ es el Hamiltoniano unielectrónico de Kohn-Sham (KS). Se puede relacionar el sistema de referencia ficticio de $\mathrm{KS}$ con el sistema real escribiendo el Hamiltoniano $H_{\lambda} \equiv T+\sum_{i} v_{\lambda}\left(r_{i}\right)+\lambda V_{e e}$, donde el parámetro $\lambda$ toma valores de 0 (sin repulsiones electrónicas, el sistema de referencia), a 1 (el sistema real); y $v_{\lambda}$ se define como un potencial externo que hace que la densidad electrónica del estado fundamental del sistema con Hamiltoniano $H_{\lambda}$ sea igual al estado fundamental del sistema real.

Como el sistema de referencia $s$ consta de partículas no interactuantes, la función de onda del estado fundamental del sistema de referencia $\psi_{s, 0}$ debe ser un producto antisimetrizado (determinante de Slater), de los espín-orbitales de KS de más baja energía, $u_{i}^{K S}$, del sistema de referencia, donde la parte espacial $\theta_{i}^{K S}\left(r_{i}\right)$ de cada orbital de espín es una función del operador unielectrónico $h_{i}^{K S}$,

$\psi_{s, 0}=\left|u_{1} u_{2} \cdots u_{n}\right|, \quad u_{i}=\theta_{i}^{K S}\left(r_{i}\right) \sigma_{i}$

$h_{i}^{K S} \theta_{i}^{K S}=\varepsilon_{i}^{K S} \theta_{i}^{K S}$

donde $\sigma_{i}$ es una función de espín ( $\alpha$ ó $\beta$ ), y las $\varepsilon_{i}^{K S}$ son las energías orbitales de Kohn-Sham.

Para un estado fundamental de capa cerrada, los electrones estarán apareados en los orbitales de Kohn-Sham. Cabe resaltar que, aunque es común en la literatura referirse al conjunto $\{\theta\}$ como orbitales de $K$-S, y así lo hemos hecho en esta sección, en el trabajo original de Kohn y Sham los autores hablan de funciones auxiliares para definir el conjunto $\{\theta\}$ y no utilizan la palabra orbital.

Kohn y Sham reescribieron las ecuación (2.12) de Hohenberg-Kohn como sigue. Definimos $\Delta T$,

$\Delta T[\rho] \equiv T[\rho]-T_{s}[\rho]$

como la diferencia entre la energía cinética electrónica del estado fundamental del sistema real y del sistema de referencia de electrones no interactuantes con densidad electrónica igual a la del sistema real. Se omite en ésta y las ecuaciones siguientes el subíndice 0 de $\rho$.

Sea

$\Delta V_{e e}[\rho]=V_{e e}[\rho]-\frac{1}{2} \iint \frac{\rho\left(r_{1}\right) \rho\left(r_{2}\right)}{r_{12}} d r_{1} d r_{2}$

la parte no clásica de la interacción electrónica. La cantidad $\frac{1}{2} \iint \frac{\rho\left(r_{1}\right) \rho\left(r_{2}\right)}{r_{12}} d r_{1} d r_{2}$ es la expresión clásica, de la energía de repulsión electrostática interelectrónica si los electrones están dispersos en una distribución continua de carga con densidad electrónica $\rho$.

Con las ecuaciones (2.16) y (2.17), (2.12) se convierte en: 
$E_{v}[\rho]=\int \rho(r) v(r) d r+T_{s}[\rho]+\frac{1}{2} \iint \frac{\rho\left(r_{1}\right) \rho\left(r_{2}\right)}{r_{12}} d r_{1} d r_{2}+\Delta T[\rho]+\Delta V_{e e}[\rho]$

Los funcionales $\Delta T$ y $\Delta V_{e e}$ son desconocidos. Se define el funcional de la energía de correlación y de intercambio $E_{x c}[\rho]$, como:

$E_{x c}[\rho] \equiv \Delta T[\rho]+\Delta V_{e e}[\rho]$

tenemos,

$E_{0}=E_{v}[\rho]=\int \rho(r) v(r) d r+T_{s}[\rho]+\frac{1}{2} \iint \frac{\rho\left(r_{1}\right) \rho\left(r_{2}\right)}{r_{12}} d r_{1} d r_{2}+E_{x c}$

De esta manera, Kohn y Sham resuelven el problema de la indeterminación de $T[\rho]$.

La clave para efectuar un cálculo KS DFT de propiedades químicas con precisión es tener una buena aproximación a $E_{x c}$.

Por (2.20) vemos que necesitamos obtener la expresión para la densidad electrónica del estado fundamental. Como el sistema ficticio de electrones no interactuantes se caracteriza por tener la misma densidad electrónica que la del estado fundamental del sistema real $\rho_{s}=\rho_{0}$; y como la densidad de probabilidad electrónica de un sistema de $n$-partículas cuya función de onda es un determinante de Slater de los orbitales de espín $u_{i}^{K S}=\theta_{i}^{K S} \sigma_{i}$, está dada por $\sum_{i=1}^{n}\left|\theta_{i}^{K S}\right|^{2}$, entonces,

$\rho=\rho_{s}=\sum_{i=1}^{n}\left|\theta_{i}^{K S}\right|^{2}$

Podemos, por lo tanto, usando las ecuaciones anteriores, obtener $E_{0}$ a partir de $\rho$ si podemos obtener los orbitales KS $\theta_{i}^{K S}$ y si conocemos el funcional de $E_{x c}$.

El teorema variacional de Hohenberg-Kohn nos dice que podemos obtener la energía del estado fundamental variando $\rho$ (si $\int \rho d r=N$ ), de forma que minimice el funcional $E[\rho]$. De manera similar, en lugar de variar $\rho$, se puede variar los orbitales KS $\theta_{i}^{K S}$, que determinan $\rho$ por (2.21). Se puede demostrar que los orbitales de Kohn-Sham que minimizan la energía del estado fundamental satisfacen:

$\left[-\frac{1}{2} \nabla_{1}^{2}-\sum_{\alpha} \frac{Z_{\alpha}}{r_{1 \alpha}}+\int \frac{\rho\left(r_{2}\right)}{r_{12}} d r_{2}+v_{x c}(r)\right] \theta_{i}^{K S}(r)=\varepsilon_{i}^{K S} \theta_{i}^{K S}(r)$

donde el potencial de correlación y de intercambio $v_{x c}$, se obtiene como la derivada del funcional de la energía de correlación y de intercambio: 
$v_{x c}(r) \equiv \frac{\delta E_{x c}[\rho(r)]}{\delta \rho(r)}$

Es importante notar, también, que los orbitales de Kohn-Sham $\theta_{i}^{K S}$ son funciones para el sistema de referencia ficticio de electrones no interactuantes, estrictamente hablando no tienen otro significado físico que permitir que se calcule $\rho$ del estado fundamental a partir de la expresión (2.21).

A partir de (2.14) y (2.15), formas alternativas de escribir (2.22), son:

$\left[-\frac{1}{2} \nabla_{1}^{2}+v(r)\right] \theta_{i}^{K S}=\varepsilon_{i}^{K S} \theta_{i}^{K S}(r)$

$h^{K S} \theta_{i}^{K S}=\varepsilon_{i}^{K S} \theta_{i}^{K S}$

Si se conoce $E_{x c}[\rho]$, su derivada del funcional se obtiene por (2.23), y de esta forma se conoce $v_{x c}$. El problema es que no se sabe cuál es la forma funcional exacta de $E_{x c}[\rho]$, por lo que $v_{x c}$ es también desconocido. En el límite de un conjunto de bases infinito, DFT es una teoría exacta, pero las ecuaciones relevantes deben ser resueltas aproximadamente porque un operador clave tiene forma desconocida. Como resultado, esfuerzos considerables se han hecho y se hacen para encontrar funcionales de la densidad que aproximen razonablemente $E_{x c}$. Además, cómo la densidad se necesita para calcular los elementos de la ecuación (2.22), pero se determina usando los orbitales derivados de la solución de esta misma ecuación, el proceso de Kohn-Sham debe ser llevado a cabo usando un procedimiento iterativo.

\subsection{Funcionales de intercambio y correlación}

Como se ha enfatizado previamente, $E_{x c}$ no solo considera la diferencia entre la repulsión electrón-electrón clásica y mecano-cuántica, sino también incluye la diferencia en energía cinética entre el sistema ficticio no interactuante y el sistema real ${ }^{38,39,40}$. En la práctica, sin embargo, la mayoría de los funcionales modernos no intentan calcular esta porción explícitamente. En lugar de eso, ignoran el término o intentan construir una función cavidad que es análoga a la de la ecuación (2.6), excepto en el hecho de que también incorpora la diferencia en energía cinética entre el sistema ficticio no interactuante y el sistema real. Además, en muchos funcionales aparecen parámetros empíricos, que necesariamente introducen alguna corrección a la energía cinética si se basan en experimentos.

Al discutir la naturaleza de varios funcionales, es conveniente adoptar una notación comúnmente usada en el ámbito. Por ejemplo, la dependencia funcional de $E_{x c}$ en la densidad electrónica, se expresa como una interacción entre la densidad electrónica y la densidad de energía por partícula $\varepsilon_{x c}$ que depende de la densidad electrónica, a saber. 
$E_{x c}[\rho(r)]=\int \rho(r) \varepsilon_{x c}[\rho(r)] d r$

La densidad de energía $\varepsilon_{x c}$ siempre se trata como una suma de contribuciones individuales de intercambio y correlación. Para evitar confusiones, se aclara que la densidad electrónica es una densidad por unidad de volumen, mientras que la densidad de energía es una densidad por partícula. En cualquier caso, dentro de este formalismo, es claro a partir de la ecuación (2.9), que la densidad de energía de intercambio de Slater ${ }^{35}$, por ejemplo, es:

$\varepsilon_{x}[\rho(r)]=-\frac{9 \alpha}{8}\left(\frac{3}{\pi}\right)^{1 / 3} \rho^{1 / 3}(r)$

Otra convención expresa a la densidad electrónica en términos de un radio efectivo tal que exactamente un electrón estará contenido dentro de la esfera definida por ese radio y tendrá la misma densidad en todo el espacio como en el centro de la esfera,

$r_{s}(r)=\left(\frac{3}{4 \pi \rho(r)}\right)^{1 / 3}$

Por último, se ha ignorado el tema del espín hasta este punto. El espín puede ser tratado fácilmente dentro de la DFT, simplemente se necesita utilizar funcionales individuales para las densidades $\alpha$ y $\beta$. Las densidades de espín en cualquier posición se expresan usualmente en términos de $\zeta$, la polarización de espín normalizada,

$\zeta(r)=\frac{\rho^{\alpha}(r)-\rho^{\beta}(r)}{\rho(r)}$

entonces la densidad de espín $\alpha$ es simplemente la mitad del producto de $\rho$ y $(\zeta+1)$, y la densidad de espín $\beta$ es la diferencia entre ese valor y $\rho$ total.

\section{Aproximación local de la densidad}

El término aproximación local de la densidad (LDA, local density approximation), fue usado originalmente para indicar cualquier teoría del funcional de la densidad donde el valor $\varepsilon_{x c}$ en cualquier posición r pueda ser calculado exclusivamente a partir del valor de $\rho$ en esa posición, es decir, el valor "local" de $\rho$. En principio, entonces, uno de los requerimientos de $\rho$ es que sea unívoca en cualquier posición, o no satisfaría las condiciones de contorno. En la práctica, los únicos funcionales conformes a esta definición que han visto aplicación son aquellos derivados de análisis del gas electrónico uniforme (donde la densidad tiene el mismo valor en cualquier posición), y como resultado la LDA se usa más ampliamente para implicar que son estos funcionales de intercambio y correlación los que se usaron. 
La distinción es probablemente mejor vista con un ejemplo. A partir de la ecuación (2.9) y la discusión en la sección 2.1.2, la energía de intercambio para el gas electrónico uniforme puede ser calculada exactamente, y es dada por la ecuación (2.27), con la constante $\alpha$ igual a $\frac{2}{3}$. Sin embargo, el enfoque de Slater ${ }^{35}$ toma un valor de 1 para $\alpha$, y el modelo X $\alpha$ usa $\frac{3}{4}$. Todo estos modelos tiene la misma dependencia local en la densidad, pero sólo el primero es llamado de LDA, mientras que los otros dos se los nombra como Slater (S) y X $\alpha$.

Los métodos LDA, Slater y X $\alpha$ pueden ser extendidos al régimen de espín polarizado usando:

$$
\varepsilon_{x}[\rho(r), \zeta]=\varepsilon_{x}^{0}[\rho(r)]+\left\{\varepsilon_{x}^{1}[\rho(r)]-\varepsilon_{x}^{0}[\rho(r)]\right\}\left[\frac{(1+\zeta)^{4 / 3}+(1-\zeta)^{4 / 3}-2}{2\left(2^{1 / 3}-1\right)}\right]
$$

donde el superíndice 0 a la densidad de energía de intercambio, se da por la ecuación (2.27), con el valor apropiado de la constante $\alpha$, y el superíndice 1 es la expresión análoga derivada a partir de la consideración de un gas electrónico uniforme compuesto sólo por electrones del mismo espín. Se observa que si $\zeta=0$ (sistema no polarizado) el segundo término de la ecuación (2.30) es cero. Los sistemas que incluyen la polarización de espín (es decir, sistemas de capa abierta), como lo son la mayoría de los agregados estudiados en este trabajo, deben usar el formalismo de espín polarizado, que generalmente se llama "aproximación local de la densidad de espín" (LSDA, local spin density aproximation).

En cuanto a la densidad de energía de correlación, aún para el simple caso del gas electrónico uniforme, ninguna derivación analítica de este funcional ha sido probada como posible. Sin embargo, Ceperley y Alder ${ }^{41}$, usando técnicas de Monte Carlo calcularon la energía total para gases electrónicos uniformes de varias densidades distintas, obteniendo una exactitud numérica alta. Fueron capaces de extraer la energía de correlación de estos sistemas. Vosko, Wilk y Nusair $^{42}$, diseñaron posteriormente funcionales locales de la densidad que se ajustaban a estos resultados. En particular, propusieron un funcional de espín polarizado completamente análogo a la ecuación (2.30) en términos de su dependencia con $\zeta$, pero con las densidades no polarizada y totalmente polarizada expresadas de la siguiente manera (en términos de $\mathrm{r}_{\mathrm{s}}$, en vez de $\rho$ ver ecuación (2.28)):

$$
\begin{aligned}
\mathcal{E}_{c}^{i}\left(r_{s}\right)= & \frac{A}{2}\left\{\ln \frac{r_{s}}{r_{s}+b \sqrt{r_{s}}+c}+\frac{2 b}{\sqrt{4 c+b^{2}}} \tan ^{-1}\left(\frac{\sqrt{4 c-b^{2}}}{2 \sqrt{r_{s}}+b}\right)\right. \\
& \left.-\frac{b x_{0}}{x_{0}^{2}+b x_{0}+c}\left\{\ln \left[\frac{\left(\sqrt{r_{s}}-x_{0}\right)^{2}}{r_{s}+b \sqrt{r_{s}+c}}\right]+\frac{2\left(b+2 x_{0}\right)}{\sqrt{4 c-b^{2}}} \tan ^{-1}\left(\frac{\sqrt{4 c-b^{2}}}{2 \sqrt{r_{s}+b}}\right)\right\}\right\}
\end{aligned}
$$

donde diferentes conjuntos de constantes empíricas $A, x_{0}, b$ y $c$ se usan para $i=0$ e $i=1$. Vosko, Wilk y Nusair $^{42}$ propusieron varios esquemas diferentes de ajuste, variando las formas funcionales de la ecuación (2.30) y (2.31). Las dos formas que han sido más ampliamente usadas son VWN y VWN5, que dan resultados razonables en la mayoría de los casos. A los cálculos 
LSDA que emplean una combinación de las expresiones intercambio de Slater (uso de la ecuación (2.27) con $\alpha=\frac{2}{3}$ ), y la energía de correlación VWN, se los llama cálculos SVWN. A continuación se muestra un esquema de los pasos involucrados en un cálculo LSDA, Figura 2.1.

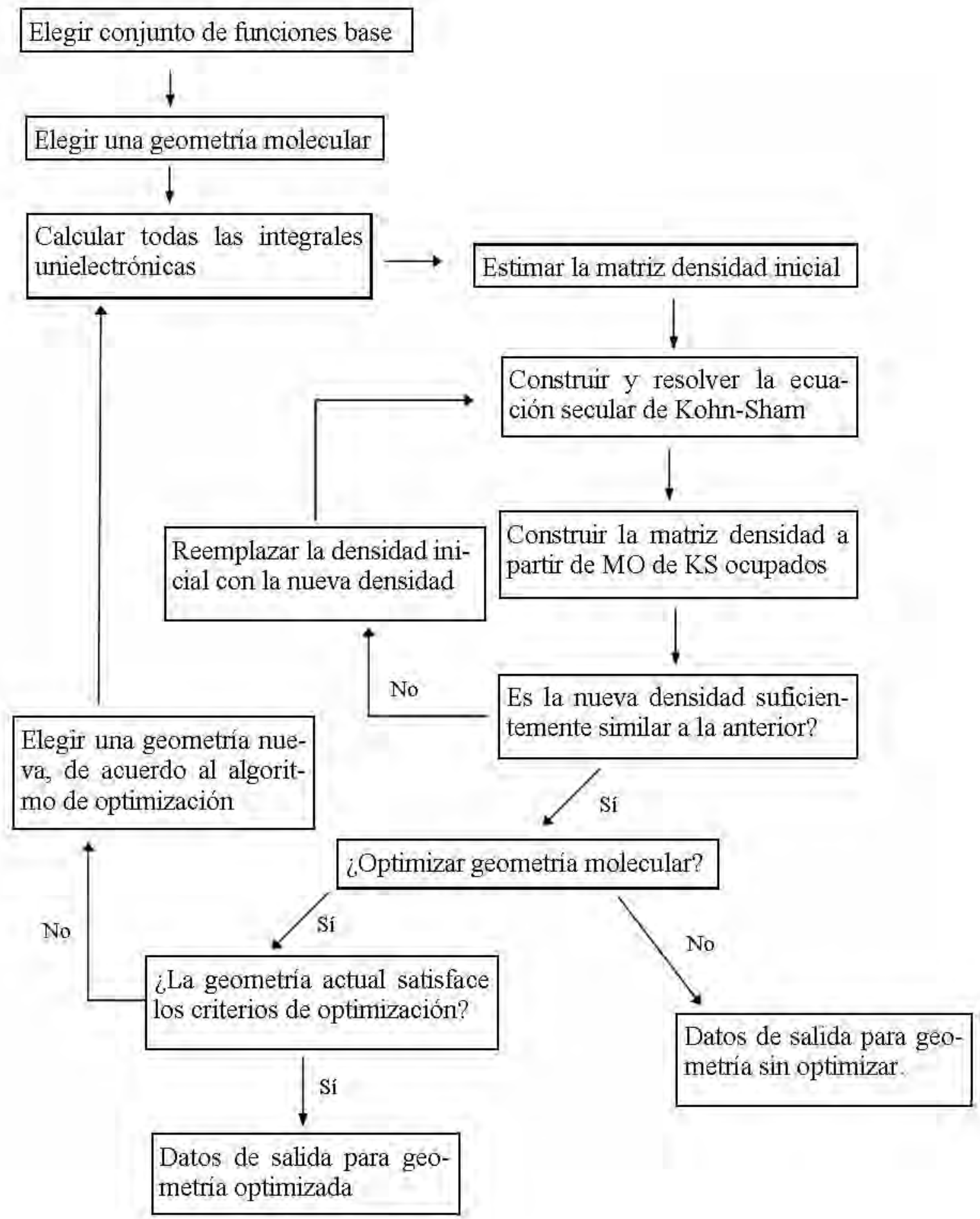

Figura 2.1. Diagrama de flujo del procedimiento autoconsistente de Kohn-Sham. 


\section{Funcionales que incluyen correcciones por gradiente de la densidad y funcionales híbridos}

Las mejoras sobre la LSDA deben considerar un gas electrónico no uniforme. Un paso en esta dirección es hacer que las energías de intercambio y correlación no dependan sólo de la densidad electrónica, sino también de sus derivadas, de su cambio en el espacio. Dichos métodos se conocen como aproximación generalizada del gradiente (GGA, generalized gradient approximation). Los métodos GGA son también llamados métodos no locales, en el sentido discutido con anterioridad.

La mayoría de los funcionales corregidos por el gradiente se construyen adicionando un término al funcional LDA, por ejemplo,

$\varepsilon_{x / c}^{G G A}[\rho(r)]=\varepsilon_{x / c}^{L D A}[\rho(r)]+\Delta \varepsilon_{x / c}(s)$

notemos que la dependencia del término de corrección es en el gradiente adimensional s definido por: $s=\frac{|\nabla \rho(r)|}{\rho^{4 / 3}(r)}$.

Becke ${ }^{43}$ propuso una corrección a la energía de intercambio que se usa ampliamente, un funcional de intercambio llamado B ó B88, que tiene incorporado un comportamiento asintótico correcto de la densidad de energía:

$$
\begin{aligned}
& \varepsilon_{x}^{B 88}=\varepsilon_{x}^{L D A}+\Delta \varepsilon_{x}^{B 88} \\
& \Delta \varepsilon_{x}^{B 88}=-\beta \rho^{1 / 3} \frac{x^{2}}{1+6 \beta x \operatorname{senh}^{-1} x}
\end{aligned}
$$

el parámetro $\beta$ se determina ajustando a datos atómicos conocidos y $x=\frac{|\nabla \rho|}{\rho^{4 / 3}}$

Los funcionales de correlación con correcciones de gradiente comúnmente usados, $E_{c}$, son entre otros el funcional de Lee, Yang y Parr (LYP) ${ }^{44}$, el funcional de correlación de Perdew (P86) ${ }^{45}$, el funcional de correlación Perdew-Wang 1991 (PW91) ${ }^{46}$, y el funcional de correlación de Becke $\mathrm{Bc} 95$ ó B96 $6^{47}$. LYP es el único que provee una cancelación exacta del error de autointeracción en sistemas unielectrónicos ${ }^{48}$. Su forma funcional es la siguiente:

$$
\begin{aligned}
& \varepsilon_{c}^{L Y P}=-a \frac{\gamma}{\left(1+d \rho^{-1 / 3}\right)}-a b \frac{\gamma e^{-c \rho^{-1 / 3}}}{9\left(1+d \rho^{-1 / 3}\right) \rho^{8 / 3}} \\
& \times\left[18\left(2^{2 / 3}\right) C_{F}\left(\rho_{\alpha}^{8 / 3}+\rho_{\beta}^{8 / 3}\right)-18 \rho t_{W}+\rho_{\alpha}\left(2 t_{W}^{\alpha}+\nabla^{2} \rho_{\alpha}\right)+\rho_{\beta}\left(2 t_{W}^{\beta}+\nabla^{2} \rho_{\beta}\right)\right] \\
& \gamma=2\left[1-\frac{\rho_{\alpha}^{2}+\rho_{\beta}^{2}}{\rho^{2}}\right] \\
& t_{W}^{\sigma}=\frac{1}{8}\left(\frac{\left|\nabla \rho_{\sigma}\right|^{2}}{\rho_{\sigma}}-\nabla \rho_{\sigma}\right)
\end{aligned}
$$


$C_{F}=\frac{3}{10}\left(3 \pi^{2}\right)^{2 / 3}$

donde los parámetros $a, b, c$ y $d$ se determinan ajustando a datos atómicos. El funcional $t_{W}$ se conoce como la densidad de energía cinética local de Weizsacker. El factor gama se vuelve cero cuando los espines están alineados $\left(\rho=\rho_{\alpha}, \rho_{\beta}=0\right)$, es decir, el funcional LYP no predice correlación de espines paralelos en dicho caso.

Se puede encontrar en la literatura una especificación completa de dichos funcionales de intercambio y correlación concatenando los acrónimos en ese orden. Entonces, por ejemplo, un cálculo BLYP combina el funcional de intercambio de Becke ${ }^{43}$ con el funcional de correlación de Lee, Yang y Parr ${ }^{44}$.

Existen también en la actualidad los funcionales híbridos, donde el B3LYP ${ }^{48,49}$ es uno de los más usados y el número 3 indica que el funcional tiene tres parámetros. Se define de la siguiente manera:

$E_{x c}^{B 3 L Y P}=\left(1-a_{0}\right) E_{x}^{L D A}+a_{0} E_{x}^{e x a c t a}+a_{x} E_{x}^{B 88}+\left(1-a_{c}\right) E_{c}^{V W N}+a_{c} E_{c}^{L Y P}$

donde $E_{x}^{\text {exacta }}$ usa la definición de HF de la energía de intercambio. Los valores del parámetro $a_{0}=0.20, a_{x}=0.72$ y $a_{c}=0.81$ se eligieron de manera de obtener un buen ajuste a las energías de atomización experimentales. Los funcionales de gradiente corregido y los funcionales híbridos no sólo dan muy buenas geometrías de equilibrio, frecuencias vibracionales y momentos dipolares, sino también, energías de atomización molecular generalmente precisas.

\subsection{Conjuntos de funciones base}

Los cálculos de orbitales moleculares son equivalentes al problema de resolver la ecuación espacial integro-diferencial

$$
f\left(r_{1}\right) \psi_{i}\left(r_{1}\right)=\varepsilon_{i} \psi_{i}\left(r_{1}\right)
$$

Se puede intentar resolver esta ecuación numéricamente. Sin embargo, no existen procedimientos prácticos para obtener soluciones numéricas para moléculas. La contribución de Roothaan fue demostrar cómo, introduciendo un conjunto de funciones de base espaciales conocidas, la ecuación diferencial puede ser convertida a un conjunto de ecuaciones algebraicas y resuelta por técnicas matriciales estándar.

Entonces, introducimos un conjunto $K$ de funciones base conocidas $\left\{\phi_{\mu}(r) \mid \mu=1,2, \ldots, K\right\}$ y expandimos los orbitales moleculares desconocidos en términos de la misma:

$$
\psi_{i}=\sum_{\mu=1}^{K} C_{\mu i} \phi_{i}
$$


Expandir una función desconocida, como puede ser un orbital molecular (MO, por sus siglas en inglés, molecular orbital), en un conjunto conocido de funciones no es una aproximación si la base está completa ${ }^{50}$. Sin embargo, un conjunto de bases completa significa que debe ser usado un número infinito de funciones, lo cual es imposible con la capacidad de cálculo actuales. Un MO desconocido puede ser pensado como una función en el infinito sistema de coordenadas que abarca el conjunto completo de bases. Cuando un conjunto finito es usado, sólo las componentes en los ejes de coordenadas correspondientes a las bases elegidas pueden ser representadas. Mientras más pequeño sea el conjunto de bases, más pobre será la representación. El tipo de funciones base utilizado influenciará también en la exactitud. Cuanto mejor represente el conjunto de bases a la función desconocida, menos funciones son necesarias para lograr el nivel de precisión deseado. El esfuerzo computacional de los métodos ab initio aumenta con el tamaño del sistema, por lo que es de gran importancia hacer el conjunto de bases lo más pequeño posible, sin comprometer la exactitud en la descripción del sistema. La expansión de los orbitales moleculares lleva a integrales de operadores mecano-cuánticos sobre las funciones base, y la facilidad con la que estas integrales se calculan, también depende del tipo de función base elegida.

Existen dos tipos de funciones base más utilizadas en cálculos de estructura electrónica: funciones de Slater, u orbitales tipo Slater (STO, Slater type orbitals) y funciones base gausianas u orbitales gausianos (GTO, Gaussian type orbitals).

\section{Funciones base tipo Slater y gausianas}

En sentido estrictamente matemático, varios tipos diferentes de funciones base $\phi_{u}$ pueden ser usados. Han sido sugeridas variedad de opciones, pero sólo dos tipos de bases se encuentran en el uso común, como se ha mencionado anteriormente, las funciones base tipo Slater y las funciones base gausianas. La función base normalizada $1 s$ de tipo Slater, centrada en $R_{A}$, tiene la forma:

$\phi_{1 s}^{S F}\left(\zeta, r-R_{A}\right)=\left(\zeta^{3} / \pi\right)^{1 / 2} e^{-\zeta\left|r-R_{A}\right|}$

donde $\zeta$ es el exponente orbital de Slater. Mientras que la función base normalizada $1 s$ de tipo gausiana, centrada en $R_{A}$, tiene la forma:

$\phi_{1 s}^{G F}\left(\alpha, r-R_{A}\right)=(2 \alpha / \pi)^{3 / 4} e^{-\alpha\left|r-R_{A}\right|^{2}}$

donde $\alpha$ es el exponente orbital gausiano. Los exponentes orbital, que son números positivos, determinan la difusibidad o tamaño de las funciones base; un exponente grande implica una función base compacta, un exponente pequeño implica una función base difusa.

Para cálculos de estructura electrónica las funciones de tipo Slater describen de manera más correcta las características cualitativas de los orbitales moleculares $\psi_{i}$ y menos funciones de Slater son necesarias en la expansión de bases de $\psi_{i}$. En estos cálculos se deben calcular cantidades muy grandes de integrales dielectrónicas $(\mu \nu \mid \lambda \sigma)$, en el orden de $\mathrm{K}^{4} / 8$. Estas integrales tienen la forma: 
$\left(\mu_{A} v_{B} \mid \lambda_{C} \sigma_{D}\right)=\int d r_{1} d r_{2} \phi_{\mu}^{A^{*}}\left(r_{1}\right) \phi_{v}^{B}\left(r_{1}\right) r_{12}^{-1} \phi_{\lambda}^{C *}\left(r_{2}\right) \phi_{\sigma}^{D}\left(r_{2}\right)$

donde $\phi_{\mu}^{A}$ es una función de base en el núcleo $A$, centrada en $R_{A}$. La integral general involucra cuatro centros diferentes: $R_{A}, R_{B}, R_{C}$ y $R_{D}$. La evaluación de estas integrales de cuatro centros es muy difícil y consume mucho tiempo con funciones de tipo Slater. Esa es la razón por la que se consideran las funciones gausianas en la práctica. Las integrales bielectrónicas pueden calcularse rápida y eficientemente con funciones gausianas, pero éstas no son funciones óptimas y tienen comportamiento funcional diferente del comportamiento funcional conocido de los orbitales moleculares. Para resolver este problema se utilizan funciones base que son combinaciones lineales de las funciones gausianas primitivas $\phi_{p}^{G F}$. Estas combinaciones lineales se llaman contracciones, y conforman las funciones gausianas contraidas (CGF, contracted Gaussian functions):

$\phi_{\mu}^{C G F}\left(r-R_{A}\right)=\sum_{p=1}^{L} d_{p \mu} \phi_{p}^{G F}\left(\alpha_{p \mu}, r-R_{A}\right)$

donde $\mathrm{L}$ es la longitud de la contracción y $d_{p \mu}$ es un coeficiente de contracción. La primitiva gausiana normalizada $\phi_{p}^{G F}$ en la función base $\phi_{\mu}^{C G F}$ tiene una dependencia funcional con el exponente orbital gausiano $\alpha_{p \mu}$. La idea detrás del uso de gausianas contraídas es elegir de antemano la longitud de contracción, los coeficientes de contracción y los exponentes de contracción que ajustarán en el lado derecho de (2.41) a un conjunto de funciones base $\phi_{\mu}^{C G F}$ y luego usar estas funciones en cálculos de funciones de onda moleculares. Las integrales bielectrónicas $(\mu \nu \mid \lambda \sigma)$ para el conjunto de funciones de base contraídas $\left\{\phi_{\mu}^{C G F}\right\}$ pueden ser evaluadas como sumas de integrales dielectrónicas sobre las gausianas primitivas, de manera muy fácil por (2.41).

En realidad, aunque se usen funciones contraídas en el cálculo, el número de integrales dielectrónicas todavía depende de la cantidad de funciones base primitivas originales; pero, el orden del determinante a diagonalizar depende de las contraídas y de allí surge la ventaja de utilizar estas funciones, en que disminuye el orden de los determinantes, y por lo tanto el tiempo de cálculo.

\section{Clasificación de las funciones base}

Habiendo decidido el tipo de función base a utilizar (STO ó GTO), el factor más importante es el número de funciones a utilizar. El menor número de funciones posible es el conjunto base mínimo, dónde sólo las funciones suficientes son utilizadas para describir un átomo neutro. Por ejemplo, para el hidrógeno, esto significaría sólo una función de tipo s. Para la primera fila de la tabla periódica, sería dos funciones de tipo s (1s y $2 \mathrm{~s})$, y un conjunto de funciones $\mathrm{p}\left(2 \mathrm{p}_{\mathrm{x}}, 2 \mathrm{p}_{\mathrm{y}}\right.$ y $2 \mathrm{p}_{\mathrm{z}}$ ), y así sucesivamente.

La siguiente mejora en el conjunto de bases es una duplicación de todas las funciones base, produciendo una base de tipo doble zeta (DZ). El término zeta viene del hecho de que el exponente de las funciones base STO se denota frecuentemente con la letra griega $\zeta$. Una función base doble zeta emplea dos funciones tipo s para el hidrógeno (1s y $\left.1 \mathrm{~s}^{\prime}\right)$; cuatro funciones $\mathrm{s}(1 \mathrm{~s}$, 
$1 \mathrm{~s}^{\prime}, 2 \mathrm{~s}$ y $\left.2 \mathrm{~s}^{\prime}\right)$, y dos funciones $\mathrm{p}$ ( $2 \mathrm{p}$ y $\left.2 \mathrm{p}^{\prime}\right)$, para los elementos del primer período; y seis funciones $\mathrm{s}$ y cuatro funciones $\mathrm{p}$ para elementos del segundo período. Doblar el número de funciones base permite una mejor descripción del hecho de que la distribución electrónica es diferente a distintas distancias del núcleo.

Las uniones químicas ocurren entre orbitales de valencia. Doblar las funciones 1s en, por ejemplo, el carbono, permite una mejor descripción de los orbitales 1s. Sin embargo, el orbital 1s es independiente del ambiente químico, estando muy cerca del caso atómico. Una variación del tipo de base DZ sólo dobla el número de orbitales de valencia, produciendo una base de valencia dividida (split valence basis, en inglés). En cálculos reales, doblar los orbitales de la parte interna será raramente considerado, y el término DZ se usa, también, para las funciones base de valencia dividida (ó VDZ, por valence double zeta).

El próximo paso en aumentar el tamaño de la base es un tipo triple zeta (TZ). Dicha base contiene tres veces más funciones que el conjunto de bases mínimo; esto es, seis funciones de tipo s y tres funciones para elementos del primer período. Algunos de los orbitales internos pueden ser salvados, partiendo la base y produciendo una base de valencia dividida de triple zeta (VTZ, valence triple zeta). Los nombres cuádruple zeta (QZ, quadruple zeta), y quíntuple zeta (5Z), para los siguientes conjuntos de base también son usados.

Hasta ahora hemos considerado sólo funciones s y p para los elementos del primer y segundo período. En la mayoría de los casos, son necesarias funciones de mayor momento angular para describir el hecho de que la distribución electrónica a lo largo del enlace será diferente la distribución electrónica perpendicular al enlace. Este tipo de funciones se llaman funciones de polarización. De esta forma, un orbital de tipo p introduce una polarización a los orbitales de tipo $\mathrm{s}$; un orbital d puede ser usado, de manera similar, para polarizar un orbital p; etcétera.

Agregar un solo conjunto de funciones de polarización (funciones $\mathrm{p}$ a los hidrógenos y funciones $\mathrm{d}$ para átomos más pesados), a las funciones $\mathrm{DZ}$, forma un tipo de base doble zeta mas polarización (DZP). Similarmente, pueden ser agregados varios conjuntos de funciones de polarización con exponentes diferentes. Si dos conjuntos de funciones de polarización se agregan a una base sp TZ, se obtiene una base triple zeta mas dos conjuntos de polarización (TZ2P).

Por otro lado, en sistemas aniónicos o estados electrónicos excitados, donde los orbitales moleculares tienden a estar más extendidos espacialmente, el conjunto de bases no tiene la flexibilidad necesaria para que la densidad electrónica se expanda. En estos casos pueden surgir errores significativos en energías y otras propiedades moleculares. Para resolver esta limitación, los conjuntos de bases son aumentados con funciones difusas. Generalmente son funciones s, que se agregan al hidrógeno y funciones s y p para elementos más pesados.

\section{Conjuntos de base de tipo Pople}

Funciones base STO-NG

Son funciones base tipo Slater, constituidas por $N \mathrm{PGTO}^{51}$ (orbitales gausianos de tipo primitivos, del inglés primitive gaussian type orbital). Este es un conjunto de base mínima, donde los exponentes de los PGTO son determinados ajustando a los STO, en vez de optimizarlos por un procedimiento variacional. Se ha encontrado que usar más de tres PGTO para representar los STO conduce a muy pocas mejoras. Estos tipos de conjuntos de bases han sido determinados para muchos elementos de la tabla periódica. La designación para el carbono/hidrógeno es $(6 \mathrm{~s} 3 \mathrm{p} / 3 \mathrm{~s})$ $\rightarrow[2 \mathrm{~s} 1 \mathrm{p} / 1 \mathrm{~s}]$. 


\section{Conjuntos de base k-nlmG}

Éstos conjuntos de bases han sido diseñados por Pople y colaboradores, y son de tipo valencia dividida, con la $k$ del principio indicando cuántos PGTO se usaron para representar los orbitales internos. El $\mathrm{nlm}$ luego del guión indica en cuantas funciones están divididos los orbitales de valencia; y, cuantos PGTO se usaron para su representación. Dos valores $(n l)$, indican una valencia dividida doblemente (double splits valence, en inglés); mientras que tres valores $(\mathrm{nlm})$, indican valencia dividida triplemente (triple split valence).

$3-21 G$

Esta es una base de valencia dividida, donde los orbitales internos son una contracción de tres PGTO, la parte interna de los orbitales de valencia es una contracción de dos PGTO y la parte externa de la valencia se representa por un PGTO $^{52}$.

\section{6-31G}

En esta base los orbitales internos son una contracción de seis PGTO, la parte interna de los orbitales de valencia se representa por tres PGTO y la parte externa por un PGTO $^{53}$.

\section{6-311G}

Esta es una base de valencia triplemente dividida, donde los orbitales internos están contraídos en seis PGTO; la valencia está dividida en tres funciones, representadas por tres, uno y un PGTO, respectivamente ${ }^{54}$.

Como se mencionó con anterioridad, a cada una de estas bases se les puede agregar funciones difusas o de polarización. Las funciones difusas van antes de la G. Se denotan por + o ++ , donde el primer + indica un conjunto de funciones s y p difusas en los átomos pesados, y el segundo + indica que una función s difusa se agrega también a los hidrógenos. Por otro lado, las funciones de polarización son indicadas después de la $\mathrm{G}$, con designación separada para el hidrógeno y para los átomos pesados. Por ejemplo, la base $6-31+\mathrm{G}(\mathrm{d})$, es una base de valencia dividida con un conjunto de funciones $\mathrm{sp}$ difusas en los átomos pesados solamente y una sola función $\mathrm{d}$ de polarización en átomos pesados.

Si sólo se van a usar funciones de polarización, una notación alternativa es usar el símbolo *. Así, la base $6-31 \mathrm{G}^{*}$ es idéntica a $6-31 \mathrm{G}(\mathrm{d})$.

\section{Conjuntos de base consistentes con la correlación}

Los conjuntos de base consistentes con la correlación (cc, del inglés correlation consistent), fueron diseñados para recobrar la energía de correlación de los electrones ${ }^{55}$. El nombre consistente con la correlación se refiere al hecho de que los conjuntos de base están diseñados para que las funciones de polarización que contribuyen en proporciones similares a la energía de correlación sean incluidas en la misma etapa, independientemente del tipo de función. Por ejemplo, la primera función d de polarización provee una disminución de energía importante, debido a efectos de correlación, pero la contribución de la segunda función d es similar a la de la primera función $\mathrm{f}$. La disminución en energía de la tercera función d es similar a aquella de la segunda función $\mathrm{f}$ y la primera función $\mathrm{g}$. Entonces, el agregado de funciones de polarización debe ser realizado en el siguiente orden: $1 \mathrm{~d}, 2 \mathrm{~d} 1 \mathrm{f}$ y $3 \mathrm{~d} 2 \mathrm{f} 1 \mathrm{~g}$. El aumento en el número de funciones de polarización debe ir acompañado por un aumento en el número de funciones que describen el espacio de valencia. Se encuentran disponibles varios tamaños de conjuntos de bases cc, dependiendo del número total de funciones contraídas. Son conocidas por sus acrónimos: cc- 
pVDZ, cc-pVTZ, cc-pVQZ, cc-pV5Z y cc-pV6Z, por sus siglas en inglés correlation consistent polarizad valence double/triple/quadruple/quintuple/sextuple zeta. Se muestra la composición en la Tabla 2.1.

Tabla 2.1. Conjuntos de base consistentes con la correlación.

\begin{tabular}{|c|c|c|}
\hline Base & Funciones primitivas & Funciones contraídas \\
\hline cc-pVDZ & $9 s, 4 p, 1 d / 4 s, 1 p$ & $3 s, 2 p, 1 d / 2 s, 1 p$ \\
\hline cc-pVTZ & $10 s, 5 p, 2 d, 1 f / 5 s, 2 p, 1 d$ & $4 s, 3 p, 2 d, 1 f / 3 s, 2 p, 1 d$ \\
\hline cc-pVQZ & $12 s, 6 p, 3 d, 2 f, 1 g / 6 s, 3 p, 2 d, 1 f$ & $5 s, 4 p, 3 d, 2 f, 1 g / 4 s, 3 p, 2 d, 1 f$ \\
\hline cc-pV5Z & 14s,9p,4d,3f,2g,1h/8s,4p,3d,2f,1g & $6 s, 5 p, 4 d .3 f, 2 g, 1 h / 5 s, 4 p, 3 d, 2 f, 1 g$ \\
\hline cc-pV6Z & $\begin{array}{c}16 \mathrm{~s}, 10 \mathrm{p}, 5 \mathrm{~d}, 4 \mathrm{f}, 3 \mathrm{~g}, 2 \mathrm{~h}, 1 \mathrm{i} / \\
10 \mathrm{~s}, 5 \mathrm{p}, 4 \mathrm{~d}, 3 \mathrm{f}, 2 \mathrm{~g}, 1 \mathrm{~h}\end{array}$ & $\begin{array}{c}7 \mathrm{~s}, 6 \mathrm{p}, 5 \mathrm{~d}, 4 \mathrm{f}, 3 \mathrm{3g}, 2 \mathrm{~h}, 1 \mathrm{i} / \\
6 \mathrm{~s}, 5 \mathrm{p}, 4 \mathrm{~d}, 3 \mathrm{ff}, 2 \mathrm{~g}, 1 \mathrm{~h}\end{array}$ \\
\hline
\end{tabular}

Este tipo de bases puede ser aumentado por funciones difusas adicionales, adicionando el prefijo aug- al acrónimo correspondiente ${ }^{56}$. El aumento consiste en adicionar una función extra con un menor exponente para cada momento angular, por ejemplo, la base aug-cc-pVDZ tiene funciones $1 \mathrm{~s}-, 1 \mathrm{p}$ - y 1d- adicionales. La mayor ventaja de estas bases es la habilidad para generar una secuencia de conjuntos de base que converge hacia el límite de las bases (límite dado por el uso de un número infinito de funciones); y la mayor desventaja es que con el aumento en la calidad de la base aumenta el número de funciones base.

\subsection{Análisis poblacional basado en el potencial electrostático molecular}

Una actividad continua en química teórica ha sido la búsqueda de métodos mejorados para elucidar y predecir el comportamiento reactivo de moléculas y otras especies químicas. Algunos índices de reactividad en los últimos tiempos, han sido diseñados y usados para proveer alguna medida de las actividades químicas de varios sitios y regiones moleculares. Dentro de dichos índices están las cargas atómicas, órdenes de enlace, densidades electrónicas, orbitales moleculares de frontera, y energías de estados de transición. Nos enfocaremos en el potencial electrostático molecular (MEP, Molecular Electrostatic Potential) ${ }^{57,58}, V(\vec{r})$, que se compone, en el espacio que rodea el sistema, por sus núcleos y electrones; $V(\vec{r})$, es una herramienta útil para el estudio de la reactividad. Al contrario de muchos otros índices de reactividad, el MEP es una propiedad física real que puede ser determinada experimentalmente por métodos de difracción. $V(\vec{r})$ ha sido extensivamente usado para interpretar y predecir el comportamiento reactivo de una amplia variedad de sistemas químicos en reacciones nucleofílicas y electrofílicas, y en el estudio de procesos de reconocimiento biológico e interacciones de uniones hidrógeno.

\section{Definición y significado}

Cualquier distribución de carga eléctrica, como los electrones y núcleos de una molécula, crea un potencial eléctrico $V(\vec{r})$ en el espacio circundante. $V(\vec{r})$ es el potencial de la molécula debido a la interacción con una carga eléctrica localizada en $\vec{r}$. Una carga puntual $\pm Q$, que se aproxima, interactuará con este potencial con una energía igual a exactamente $\pm Q V(\vec{r})$, donde $\vec{r}$ es la 
posición de la carga puntual. Entonces, una carga puntual positiva será atraída a aquellas regiones en dónde $V(\vec{r})$ es negativo, dado que esto lleva a una energía de interacción negativa y estabiliza el sistema; y será repelida de las regiones de potencial positivo, en la cual la energía de interacción es positiva y desestabilizante.

Un conocimiento del potencial eléctrico alrededor de una molécula ayudará considerablemente a interpretar su comportamiento reactivo hacia especies cargadas (aún, cualitativamente, cuando son mucho más que cargas puntuales), y en predecir los sitios de la molécula en los cuales será más factible que reaccione. De hecho, el potencial eléctrico es una herramienta muy importante para la elucidación de propiedades reactivas moleculares. En estas aplicaciones, el sistema es normalmente visto como teniendo una distribución de carga electrónica estática alrededor de un arreglo nuclear rígido, entonces el término "potencial electrostático" es más adecuado para $V(\vec{r})$. Si una molécula tiene una función de densidad electrónica $\rho(\vec{r})$, entonces su potencial electrostático en cualquier punto $\vec{r}$ está dado por:

$V(\vec{r})=\sum_{A} \frac{Z_{A}}{\left|\vec{R}_{A}-\vec{r}\right|}-\int \frac{\rho\left(\vec{r}^{\prime}\right) d \vec{r}^{\prime}}{\left|\vec{r}^{\prime}-\vec{r}\right|}$

$Z_{A}$ es la carga del núcleo $A$, localizado en $\vec{R}_{A}$. El primer término a la derecha de la ecuación (2.42), que es positivo, representa la contribución del núcleo; el segundo término aporta el efecto de los electrones, y es negativo.

Una cualidad importante del potencial electrostático es que es una propiedad física real, como lo evidencia el hecho de que puede ser determinado experimentalmente por técnicas de difracción. Está definido de manera rigurosa y no ambigua por la ecuación (2.42), y tiene un significado físico muy claro: expresa el efecto eléctrico neto de los electrones y núcleos de un sistema en el espacio circundante.

\section{MEP como descriptor de reactividad}

La descripción de la reactividad molecular es una de las metas más importantes de la química cuántica. Mientras los métodos actuales pueden ser usados para calcular los caminos de reacción de sistemas no muy complejos, son, además, todavía útiles para interpretar y predecir la reactividad molecular vía descriptores que reflejan la distribución electrónica de las moléculas individuales. Obviamente, las propiedades de moléculas aisladas no dan toda la información de una reacción dada.

Bonaccorsi, Scrocco y Tomasi ${ }^{57,58}$ proponen utilizar al MEP como un descriptor molecular donde una de las especies que reaccionan está considerada explícitamente y la otra está representada por una carga de prueba, siendo su energía de interacción evaluada sin efectos de polarización o rearreglos nucleares. Este enfoque se volvió rápidamente muy exitoso para el estudio de reacciones electrofílicas, la unión hidrógeno $\mathrm{y}$, aunque no tan directamente como para un ataque electrofílico, fue luego modificado para el caso de reacciones nucleofílicas. Las cantidades que permiten calcular el potencial electrostático se obtienen a partir de la función de onda del sistema, que se calcula a nivel semiempírico, ab-initio, o DFT. Es también importante encontrar las cargas parciales derivadas del cálculo del potencial electrostático (cargas ESP), que mostrarán cuantitativamente cómo el sistema químico actuará ante la especie que se acerca. Este tipo de 
cargas son actualmente muy usadas en biomoléculas para cálculos más complejos tales como QM/MM y se obtienen fácilmente con los programas disponibles.

\section{Limitaciones del uso del potencial electrostático como indicador de reactividad}

Aunque el potencial electrostático ha probado ser una técnica efectiva para analizar y predecir el comportamiento reactivo molecular, es importante reconocer algunas limitaciones intrínsecas en su uso. $V(\vec{r})$, como está definido en la ecuación (2.42), es una propiedad de una molécula $\mathrm{M}$ en ese estado. $V(\vec{r})$ no tiene en cuenta los cambios que ocurren en $\mathrm{M}$ a medida que interactúa con algunas especies que se aproximan (por ejemplo, polarización y transferencia de carga), ni refleja de ninguna manera la naturaleza de las mismas. Uno puede usar, por supuesto, varios enfoques, incluyendo técnicas de perturbación, para estimar la contribución de dichos factores. Sin embargo, esto va más allá del uso del potencial electrostático en sí mismo como una guía de reactividad molecular.

Debido a estas limitaciones, el potencial electrostático es más útil como una guía en las etapas tempranas de una reacción, en la cual la especie atacante no está muy cerca aún de la molécula $\mathrm{M}$ y su mutua polarización, transferencia de carga, etc., son relativamente insignificantes.

El potencial electrostático de una molécula es indicativo de lo que encuentra una especie molecular que se está acercando a la vecindad de $\mathrm{M}$, antes de que una polarización significativa de la última haya ocurrido. El potencial es muy importante para responder a las preguntas de cuál es el mejor camino para acercarse y cuál es la región preferida de ataque ${ }^{57}$.

\subsection{Orbitales naturales de enlace}

El concepto de orbitales naturales ${ }^{50}$ puede ser usado para distribuir electrones en orbitales atómicos y moleculares, y por consiguiente, para derivar cargas atómicas y uniones moleculares. La idea en los análisis de orbitales atómicos naturales (NAO, natural atomic orbitals), y orbitales naturales de enlace (NBO, natural bond orbital), desarrollados for F. Weinholt ${ }^{59}$ y colaboradores es usar la matriz de densidad electrónica para definir la forma de los orbitales atómicos en el ambiente molecular, y derivar uniones moleculares a partir de la densidad electrónica entre átomos.

Supongamos que las funciones base han sido ordenadas de manera que los orbitales localizados en el centro A están antes que los localizados en el centro B, que están antes que aquellos en el centro $\mathrm{C}$, etc.

$\chi_{1}^{A}, \chi_{2}^{A}, \chi_{3}^{A}, \ldots, \chi_{k}^{B}, \chi_{k+1}^{B}, \chi_{k+2}^{B}, \ldots, \chi_{n}^{C}, \chi_{n+1}^{C}, \chi_{n+2}^{C}, \ldots$

La matriz densidad puede escribirse en términos de bloques de funciones base pertenecientes a un centro específico: 


$$
D=\left(\begin{array}{cccc}
D^{A A} & D^{A B} & D^{A C} & \ldots \\
D^{A B} & D^{B B} & D^{B C} & \ldots \\
D^{A C} & D^{B C} & D^{C C} & \ldots \\
\ldots & \ldots & \ldots & \ldots
\end{array}\right)
$$

Los orbitales naturales atómicos para el átomo $\mathrm{A}$ en el ambiente molecular pueden definirse como aquellos que diagonalizan el bloque $D^{A A}$, los NAO para el átomo B son aquellos que diagonalizan el bloque $D^{B B}$, etc. Estos NAO generalmente no serán ortogonales. Para lograr una distribución de electrones bien definida, los orbitales deberán ortogonalizarse. El procedimiento es el siguiente:

1) Cada uno de los bloques atómicos en la matriz densidad se diagonaliza para producir un conjunto de NAO no ortogonales, a menudo llamado pre-NAO.

2) Los pre-NAO fuertemente ocupados de cada centro se hacen ortogonales a cada pre-NAO fuertemente ocupado en los otros centros.

3) Los pre-NAO poco ocupados en cada centro se hacen ortogonales a los NAO fuertemente ocupados en el mismo centro.

4) Los NAO débilmente ocupados se hacen ortogonales a todos los NAO poco ocupados en los otros centros.

El conjunto final de orbítales ortogonales se llaman simplemente NAO, y los elementos diagonales de la matriz densidad en esta base son las poblaciones de los orbitales. Sumando todas las contribuciones de los orbítales que pertenecen a un centro específico se obtiene la carga atómica. La ventaja de los NAO es que se definen a partir de la matriz densidad, garantizando que la ocupación electrónica sea entre 0 y 2 ; y que convergen a valores bien definidos a medida que el tamaño de la base aumenta. También, el análisis puede ser realizado para funciones de onda correlacionadas.

Una vez que la matriz densidad ha sido transformada en la base NAO, los enlaces entre átomos se definen a partir de los bloques no diagonales. El procedimiento incluye los siguientes pasos:

1) Los orbítales atómicos naturales para un bloque atómico en la matriz densidad que tienen números de ocupación muy cercanos a 2 (>1.999), se identifican como orbitales internos (core orbitals). Sus contribuciones a la matriz densidad se remueven.

2) Los orbitales atómicos naturales en la matriz densidad que tienen números de ocupación grandes $(>1.90)$, se identifican como pares libres (lone pair orbitals). Sus contribuciones a la matriz densidad también se remueven.

3) Cada par de átomos ( $\mathrm{AB}, \mathrm{AC}, \mathrm{BC}, \ldots)$, se considera y se diagonalizan los bloques $2 \times 2$ de la matriz densidad (con las contribuciones de orbitales internos y de pares libres removidas). Los orbitales naturales de enlace (NBO) son identificados como autovalores que tienen números de ocupación grandes (usualmente mayores a 1.90).

El resultado es tal que, excepto para contribuciones muy pequeñas de otros orbitales atómicos para asegurar ortogonalidad, todos los NAO y los orbitales Rydberg se describen usando conjuntos de bases de orbitales atómicos de un solo átomo, y todos los NBO se describen usando conjuntos de base de orbitales atómicos de dos átomos. Se debe realizar trabajo adicional en 
casos donde se presentan efectos de delocalización tales como efectos de resonancia que requieren que los orbitales estén delocalizados en más de dos átomos. Una vez que se ha identificado a los NBO, pueden ser escritos como combinaciones lineales de los NAO, mostrando los orbitales atómicos involucrados en los enlaces. El análisis NBO provee un esquema de orbitales muy cercano a una estructura de Lewis para una molécula. Sin embargo, debe ser resaltado que el análisis NBO es sólo un modelo conceptual, dado que está basado en orbitales, y se deben esperar limitaciones en su uso en casos en que las especies químicas estén representadas pobremente por estructuras de Lewis ${ }^{32}$.

\subsection{Metodología de trabajo}

El presente trabajo tiene como objeto el estudio de las propiedades geométricas, electrónicas y vibracionales, de agregados de estequiometría $\mathrm{X}_{n} \mathrm{Y}_{m}, \mathrm{X}=\mathrm{B}, \mathrm{Al} ; \mathrm{Y}=\mathrm{N}, \mathrm{P} ; n=1-4$ y $m=1-4$ (n $+\mathrm{m} \leq 5)$, con el fin de contribuir a la comprensión de las propiedades de películas delgadas conformadas por estas aleaciones $\mathrm{y}$, eventualmente, proveer información relevante para el desarrollo de nuevos materiales con propiedades electrónicas particulares. Asimismo, se busca conocer el patrón de crecimiento de dichos agregados, teniendo en cuenta el camino de aumento de la energía de enlace.

Las propiedades geométricas, electrónicas, y vibracionales de los agregados binarios mencionados, se estudiaron utilizando herramientas de la teoría del funcional de la densidad $^{30,31,37}$, como ser el funcional híbrido de tres parámetros de Becke, que combinado con el funcional de correlación de Lee, Yang y Parr, da lugar al funcional conocido como B3LYP. Los conjuntos de funciones base utilizados fueron de calidad triple-zeta más polarización en el espacio de valencia, de tipo correlation consistent, cc-pVTZ. Los cálculos se llevaron a cabo utilizando el paquete de programas Gaussian, en su versión $03^{60}$.

Con el objeto de realizar una contribución novedosa al estudio de los agregados de interés, se realizó un estudio del crecimiento de los mismos, justificando el mismo mediante mapas de potencial electrostático. En el caso de agregados de capa abierta, el análisis se complementó con densidades de espín. Es importante destacar que, hasta el momento, no se han encontrado en literatura estudios teóricos de este tipo.

En el caso de agregados de $\mathrm{Al} / \mathrm{N}$ y $\mathrm{B} / \mathrm{N}$, se realizaron análisis preliminares utilizando poblaciones de Mulliken $^{61}$, gráficos COOP y DOS, índices de DFT conceptual y métodos de análisis de la función de onda (Átomos en Moléculas, Función de Localización Electrónica), cuyos aspectos formales se desarrollan en el apéndice 1.

Todas las representaciones gráficas de los agregados de este trabajo se hicieron con el software gOpenMol $^{62}$.

El detalle del trabajo es el siguiente:

1. Determinación de la geometría y estructura electrónica correspondiente al estado fundamental de las especies binarias $\mathrm{X}_{n} \mathrm{Y}_{m}, \mathrm{X}=\mathrm{B}, \mathrm{Al} ; \mathrm{Y}=\mathrm{N}, \mathrm{P} ; n=1-4$ y $m=1-4(\mathrm{n}+\mathrm{m} \leq 5)$. El estudio se realizó sobre los agregados neutros, sin restricciones de simetría, optimizando también la multiplicidad. En algunos casos se realizó una optimización previa de geometría a un nivel de teoría más bajo, SVWN/3-21G, con el fin de lograr mejores hesianos iniciales.

2. Comprobación de la calidad de mínimos de las superficies de energía potencial de las estructuras determinadas anteriormente por medio de la diagonalización de la matriz hesiana obtenida con el mismo nivel de teoría que las optimizaciones de geometría. En los casos en que 
se obtuvieron autovalores del hesiano negativos, la geometría fue perturbada según el modo normal asociado y reoptimizada.

3. Obtención del camino de crecimiento de los agregados, siguiendo el aumento de la energía de atomización $\left(\mathrm{E}_{\mathrm{at}}\right)$, calculada de la siguiente manera:

$E_{a t}=\frac{x E_{x}-y E_{y}-E_{\text {cluster }}}{x+y}$

donde $E_{\text {cluster }}$ es la energía total del agregado, $E_{x}$ es la energía total del elemento del grupo III (B ó $\mathrm{Al}), E_{y}$ es la energía total del elemento del grupo $\mathrm{V}$ (N ó P), ambas energías corresponden al elemento en su estado fundamental y fueron obtenidas al mismo nivel de teoría que la estructura de equilibrio. $x$ es la cantidad total de átomos del elemento del grupo III involucrado en la formación del agregado, $y$ es la cantidad total de átomos del elemento del grupo V.

4. Obtención de cargas ESP de acuerdo al esquema de Merz-Singh-Kollman (MK) ${ }^{63}$, potenciales electrostáticos moleculares y densidades de espín mediante análisis $\mathrm{NBO}^{64}$; indicadores que, mediante su visualización y análisis, permitieron explicar el camino de crecimiento de los agregados. En la generación de los MEPs se utilizó un valor de isosuperficie de la densidad electrónica de 0.01 u.a. para los agregados constituidos por $\mathrm{Al} / \mathrm{N}, \mathrm{B} / \mathrm{N}$ y B/P y con un valor de 0.001 u.a. para los agregados formados por $\mathrm{Al} / \mathrm{P}$. 


\section{Estudio de la estructura, modos vibracionales y patrones de crecimiento de clusters de aluminio y nitrógeno}

Como la aplicación de AlN en películas delgadas sólidas para fabricar dispositivos electrónicos requiere de un entendimiento de las propiedades de clusters a nivel atómico, varios trabajos han sido realizados para lograr este objetivo. Boo y $\mathrm{Liu}^{65}$ encontraron las estructuras y energías de una estructura de $\mathrm{AlN}_{3}$, dos de $\mathrm{Al}_{3} \mathrm{~N}$, y cinco de $\mathrm{Al}_{2} \mathrm{~N}_{2}$, usando Hartree Fock y teoría de perturbaciones de segundo orden, con conjuntos de funciones base 6-31G* y cc-pVDZ, respectivamente. Nayak y colaboradores ${ }^{66}$ estudiaron la evolución de la unión en clusters de $\mathrm{Al}_{\mathrm{n}} \mathrm{N}$ $(\mathrm{n} \leq 6)$ y $\mathrm{Al}_{12} \mathrm{~N}$, aplicando teoría del funcional de la densidad y la aproximación generalizada del gradiente (DFT-GGA), usando el funcional BPW91 y el conjunto de funciones base 6-311G**. En este trabajo informan geometrías de estados fundamentales y otros estados siguientes, con sus correspondientes energías de atomización y parámetros geométricos. BelBruno ${ }^{67}$ investigó las estructuras de clusters de $\mathrm{Al}_{n} \mathrm{~N}_{\mathrm{n}}$ ( $\mathrm{n}=2-3$ ), por medio de DFT con el funcional BLYP y bases STO de calidad triple- $\zeta$. En su trabajo se calcularon seis estructuras para $\mathrm{Al}_{2} \mathrm{~N}_{2}$, tres para $\mathrm{Al}_{3} \mathrm{~N}_{3}, \mathrm{y}$ cuatro para $\mathrm{Al}_{4} \mathrm{~N}_{4}$. Qu y Bian ${ }^{68}$ estudiaron la estructura y frecuencias vibracionales de anillos simétricos de $\mathrm{Al}_{n} \mathrm{~N}_{\mathrm{n}}(\mathrm{n}=3-9)$, a nivel B3LYP/cc-pVDZ. Costales y coautores ${ }^{69}$ investigaron la evolución de las uniones químicas en nitruros del grupo III, usando DFT con el funcional de intercambio y correlación BPW91 y conjuntos de funciones base numéricas doble- $\zeta$ con funciones de polarización. Calcularon dos estructuras de $\mathrm{Al}_{2} \mathrm{~N}_{2}, \mathrm{Al}_{4} \mathrm{~N}_{2}$ y $\mathrm{Al}_{6} \mathrm{~N}_{2}$ y una estructura para los clusters $\mathrm{Al}_{3} \mathrm{~N}_{2}$ y $\mathrm{Al}_{5} \mathrm{~N}_{2}$. También estudiaron ${ }^{70}$ la estructura, estabilidad y frecuencias vibracionales de agregados poliatómicos neutros e iónicos de $\mathrm{AlN}, \mathrm{GaN}$ y $\mathrm{InN}$ hasta el trímero usando GGA y bases numéricas de calidad doble- $\zeta$ con funciones de polarización de tipo $d$, considerando un monómero como una unidad de AlN, GaN o InN. Burril y Grein ${ }^{71}$ reportaron las estructuras y uniones químicas de compuestos de los grupos III/V usando el funcional B3LYP con el conjunto de funciones base $6-311+\mathrm{G}(3 \mathrm{df})$. En su trabajo, fueron considerados los agregados $\mathrm{Al}_{2} \mathrm{~N}_{2}$ de doce geometrías diferentes. Otro trabajo utilizando DFT a nivel B3LYP/6$311+\mathrm{G}(\mathrm{d})$ fue realizado por Guo y coautores ${ }^{72}$, quienes obtuvieron la estructura y frecuencias vibracionales de agregados $\mathrm{Al}_{\mathrm{n}} \mathrm{N}_{\mathrm{m}}(\mathrm{n}=1-7 ; \mathrm{m}=1-2)$, hasta tres estructuras para cada agregado aniónico y neutro. También es importante mencionar el trabajo de Song y $\mathrm{Cao}^{73}$, quienes realizaron simulaciones de dinámica molecular usando un campo de fuerzas derivado del método conocido como Full-Potential Linear Muffin-Tin Orbital, dentro del enfoque de la aproximación local de la densidad para investigar las estructuras y energías de $\mathrm{Al}_{2} \mathrm{~N}_{3}$ y $\mathrm{Al}_{3} \mathrm{~N}_{2}$. Finalmente, Chang y colaboradores ${ }^{74}$ obtuvieron las estructuras, energías y frecuencias vibracionales de los agregados $(\mathrm{AIN})_{\mathrm{x}}(\mathrm{x}=1-4,6,12)$, con DFT a nivel BP86/6-31G(d). Como las metodologías que fueron utilizadas en estos estudios son bastante diferentes, se pueden encontrar algunas discrepancias si se comparan entre sí.

En el presente capítulo informamos los resultados obtenidos para agregados binarios de $\mathrm{Al}_{n} \mathrm{~N}_{\mathrm{m}}$, con $n=1-4, m=1-4$ y $n+m \leq 5$. Se reportan propiedades estructurales, energías de atomización y frecuencias vibracionales para los agregados más estables.

El estudio de los patrones de crecimiento se realizó conformando los agregados más allá del dímero por la adición de átomos de $\mathrm{Al}$ o $\mathrm{N}$. El análisis de los patrones de crecimiento se llevó a cabo por medio de potenciales electrostáticos moleculares (MEP), densidades de espín y cargas derivadas de potenciales electrostáticos. 


\subsection{Estructuras, energías y modos vibracionales}

\section{Dímero AlN}

Se encontró que el dímero (1) posee estado electrónico triplete (Tabla 3.1), con $\mathrm{E}_{\mathrm{at}} \mathrm{de} 1.36 \mathrm{eV} / a ́ t$, en acuerdo con las referencias 66 y 70, y con una pequeña diferencia con respecto a lo reportado por Chang y coautores ${ }^{74}$. En la Figura 3.1 se muestra la geometría del dímero, puede verse que la distancia de enlace es de $1.80 \AA$.

\section{Trímeros}

Se obtuvieron las cuatro combinaciones posibles de átomos de Al y N. El trímero (2) presenta un estado electrónico doblete con $\mathrm{E}_{\mathrm{at}}$ de $3.37 \mathrm{eV} /$ át, $1.79 \mathrm{eV} / a$ t por encima de (3), con estado electrónico cuatriplete. Los agregados (4) y (5) presentan estado electrónico sextuplete y doblete, respectivamente. Con excepción del trímero (3), los resultados obtenidos para los trímeros están de acuerdo con las referencias; aunque el confórmero (5) difiere en $0.06 \mathrm{eV} / a ́ t$ con los resultados informados en la referencia 66. Todos los trímeros resultaron ser lineales. La energía de atomización aumenta en el siguiente orden: $\mathbf{( 4 )}<\mathbf{( 3 )}<\mathbf{( 5 )}<\mathbf{( 2 )}$. Los parámetros geométricos se muestran en la Figura 3.1.

Los modos vibracionales del dímero y los trímeros se muestran en la Tabla 3.2. Se observa un buen acuerdo entre los modos calculados y resultados de otros autores, así como también el resultado experimental.

Tabla 3.1. Multiplicidades de espín $\left(\mathrm{M}_{\mathrm{S}}\right)$, energías de atomización $\left(\mathrm{E}_{\mathrm{at}}\right)$ en eV/át, del dímero y trímeros. * Calculada con corrección de punto cero.

\begin{tabular}{c|c|c} 
Agregado & $\mathbf{M}_{\mathbf{s}}$ & $\mathbf{E}_{\mathbf{a t}}$ \\
\hline $\mathbf{( 1 )}$ & 3 & 1.36 \\
& $3^{66}$ & $1.39^{66}$ \\
& $3^{70}$ & $1.37^{70}$ \\
& $3^{74}$ & $1.43^{74 *}$ \\
\hline $\mathbf{( 2 )}$ & 2 & 3.37 \\
& $2^{70}$ & \\
\hline $\mathbf{( 3 )}$ & 4 & 1.58 \\
& $2^{70}$ & \\
\hline $\mathbf{( 4 )}$ & 6 & 0.77 \\
\hline $\mathbf{( 5 )}$ & 2 & 2.56 \\
& $2^{66}$ & $2.62^{66}$ \\
& $2^{70}$ &
\end{tabular}

En la Tabla 3.1 se puede observar que, al comparar todos los agregados de tres átomos, el trímero (2) es el que tiene mayor $\mathrm{E}_{\mathrm{at}}$, aún al comparar con resultados de otros autores. 
(1)

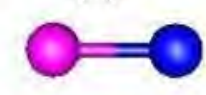

$\mathrm{r}_{\mathrm{Al}-\mathrm{N}}=1.80$
(2)

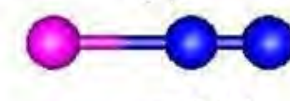

$\mathrm{r}_{\mathrm{AL}-\mathrm{N} \mathrm{S}}=2.01$

$\mathrm{r}_{\mathrm{N}-\mathrm{N}}=1.13$
(3)

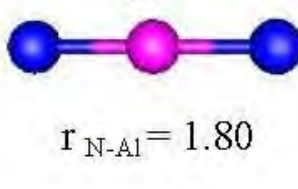

(4)

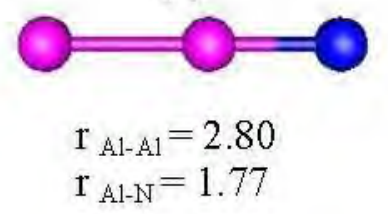

(5)

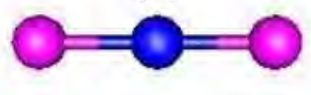

$\mathrm{r}_{\mathrm{Al}-\mathrm{N}}=1.73$

Figura 3.1. Geometrías de equilibrio para el dímero de partida y los trímeros. Las distancias (r) están expresadas en $\AA$ y los ángulos planos son $180^{\circ}$, para todos los casos.

Se puede observar, por la Figura 3.1, que la distancia Al-N está entre 1.73 y $2.01 \AA$, siendo el valor más alto cuando se encuentra seguida de una unión N-N, cuyo valor corresponde a un enlace triple. Todos los agregados de tres átomos son lineales.

Tabla 3.2. Modos normales de vibración $\left(\omega_{\mathrm{e}}\right) \mathrm{en}^{-1} \mathrm{~cm}^{-1}$ del dímero y los trímeros. El * indica que el modo es doblemente degenerado. $a$ significa que se trata de un resultado experimental.

\begin{tabular}{c|c} 
Agregado & $\boldsymbol{\omega}_{\mathbf{e}}$ \\
\hline $\mathbf{( 1 )}$ & 743 \\
& $747^{75 a}$ \\
& $710^{70}$ \\
& $752^{74}$ \\
\hline $\mathbf{( 2 )}$ & $139,297,1966$ \\
& $178,219,1887^{70}$ \\
\hline $\mathbf{( 3 )}$ & $131,150,652,736$ \\
\hline $\mathbf{( 4 )}$ & $89^{*}, 351,632$ \\
\hline $\mathbf{( 5 )}$ & $94^{*}, 527,1061$ \\
& $92,498,1014^{70}$
\end{tabular}

Respecto a los modos vibracionales (Tabla 3.2), en frecuencias bajas encontramos movimientos de tipo wagging y scissoring, en frecuencias intermedias estiramientos simétricos y asimétricos Al-Al y Al-N; y los estiramientos simétricos y asimétricos N-N se encuentran a más de $1400 \mathrm{~cm}^{-1}$.

\section{Tetrámeros}

Se calcularon los agregados estables de cuatro átomos, partiendo de los trímeros obtenidos con anterioridad. De los ocho tetrámeros encontrados, el más estable es el agregado lineal (10), de estado electrónico singlete, en concordancia con otros estudios (Tabla 3.3). Se observa que el agregado (6) es un estado degenerado de (9) con una energía de atomización $0.002 \mathrm{eV} / a ́$ t por debajo. Este resultado no se ve reflejado en la Tabla 3.3 debido a que hemos expresado las energías con dos decimales solamente. Los resultados obtenidos están de acuerdo con las 
referencias 65, 70, 71, y 74. Estructuras similares a los confórmeros (7) y (9) han sido previamente estudiadas por otros autores, como se expresa en la Tabla 3.3. Se muestran los parámetros geométricos en la Figura 3.2, donde se puede observar que todos los agregados resultaron planos, la mayoría no lineales. Los modos vibracionales se muestran en la Tabla 3.4.

Tabla 3.3. Multiplicidades de espín $\left(M_{S}\right)$ y energías de atomización $\left(E_{a t}\right)$ en eV/át, de los tetrámeros.

\begin{tabular}{c|c|c} 
Agregado & $\mathbf{M}_{\mathbf{s}}$ & $\mathbf{E}_{\mathbf{a t}}$ \\
\hline $\mathbf{( 6 )}$ & 3 & 2.99 \\
& $3^{101}$ & \\
\hline $\mathbf{( 7 )}$ & 3 & 2.44 \\
& $3^{67}$ & $2.57^{67}$ \\
& $3^{74}$ & $2.54^{74}$ \\
\hline $\mathbf{( 8 )}$ & 5 & 2.31 \\
\hline $\mathbf{( 9 )}$ & 1 & 2.99 \\
& $1^{65,70,71,74}$ & $3.14^{74}$ \\
& $1^{67}$ & $3.06^{67}$ \\
\hline $\mathbf{( 1 0 )}$ & 1 & 3.71 \\
& $1^{65}$ & \\
\hline $\mathbf{( 1 1 )}$ & 1 & 2.91 \\
\hline $\mathbf{( 1 2 )}$ & 3 & 2.09 \\
\hline $\mathbf{( 1 3 )}$ & 1 & 2.86 \\
& $1^{65,66}$ & $2.86^{66}$
\end{tabular}

Según la Tabla 3.3, el aumento en la estabilidad de los agregados, medido por el aumento de la $\mathrm{E}_{\mathrm{at}}$, se presenta de la siguiente manera: $(\mathbf{1 2})<\mathbf{( 8 )}<$ (7) $<$ (13) $<$ (11) $<$ (6) $\approx$ (9) (R) $<$ (10). También, se puede observar que al comparar todos los agregados de cuatro átomos, el tetrámero (10) es quien tiene mayor $E_{a t}$, aún al comparar con resultados de otros autores. 
(6)

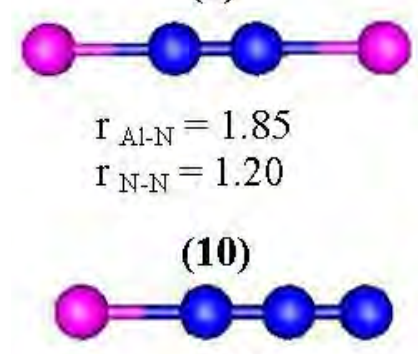

$\mathrm{r}_{\mathrm{A} 1-\mathrm{N}}=1.81$

$\mathrm{r}_{\mathrm{N} 1-\mathrm{N} 2}=1.20$

$\mathrm{r}_{\mathrm{N} 2-\mathrm{N} 3}=1.13$
(7)

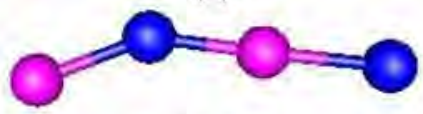

$\mathrm{r}_{\mathrm{A} 11-\mathrm{N} 1}=1.77$

$\mathrm{r}_{\mathrm{N} 1 \mathrm{-A} 12}=1.68$

$\mathrm{r}_{\mathrm{A} 12 \mathrm{-N} 2}=1.85$

$\mathrm{a}_{\mathrm{A} 11-\mathrm{N} 1-\mathrm{A} 12}=151.1$

$a_{\mathrm{N} 1-\mathrm{A} 12-\mathrm{N} 2}=178.5$
(8)

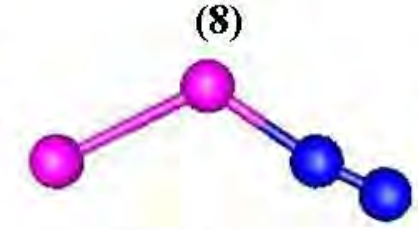

$\mathrm{r}_{\mathrm{A} 11-\mathrm{A} .12}=2.46$

$\mathrm{r}_{\mathrm{A} 12-\mathrm{N} 1}=1.94$

$\mathrm{r}_{\mathrm{N} 1-\mathrm{N} 2}=1.12$

$\mathrm{a}_{\mathrm{A} 11-\mathrm{A} 12-\mathrm{N} 1}=118.7$

$\mathrm{a}_{\text {A12-N1-N2 } 2}=171.2$

(11)

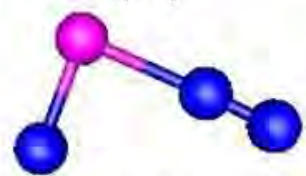

$$
\begin{aligned}
& \mathrm{r}_{\mathrm{N} 1-\mathrm{A} 11}=1.74 \\
& \mathrm{r}_{\mathrm{A} 11-\mathrm{N} 2}=2.01 \\
& \mathrm{r}_{\mathrm{N} 2 \mathrm{~N} 3}=1.10 \\
& \mathrm{a}_{\mathrm{N} 1-\mathrm{A} 11-\mathrm{N} 2}=87.1 \\
& \mathrm{a}_{\mathrm{A} 11-\mathrm{N} 2-\mathrm{N} 3}=178.3
\end{aligned}
$$

(12)

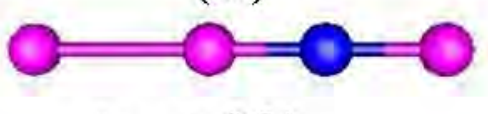

$\mathrm{r}_{\mathrm{Al}-\mathrm{Al}}=2.81$

$\mathrm{r}_{\mathrm{A} 12 \mathrm{~N}-\mathrm{N} 1}=1.69$

$\mathrm{r}_{\mathrm{N} 1-\mathrm{A} 13}=1.77$

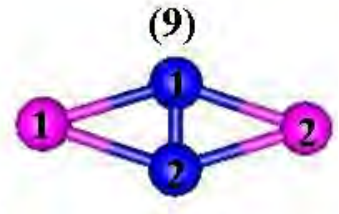

$\mathrm{r}_{\mathrm{AL}-\mathrm{N}}=2.03$

$\mathrm{r}_{\mathrm{N}-\mathrm{N}}=1.26$

$\mathrm{a}_{\mathrm{A} 1 \mathrm{-N}-\mathrm{A} \mathrm{A} 1}=143.7$

$\mathrm{a}_{\mathrm{N}-\mathrm{Al}-\mathrm{N}}=36.3$
(13)

Figura 3.2. Geometrías de equilibrio de los tetrámeros. Las distancias (r) están expresadas en $\AA$, los ángulos diedros son cero para todos los casos y los planos (a) que no se especifican, $180^{\circ}$.

La distancia Al-N se encuentra, en estos casos, entre 1.68 y $2.03 \AA$, la distancia de enlace Al-Al entre 2.46 y $2.81 \AA$, y la distancia N-N entre 1.10 y $1.20 \AA$, correspondiente a distancias triples y dobles y extendiéndose cuando se encuentra adyacente a una unión Al-N. 
Tabla 3.4. Frecuencias vibracionales $\left(\omega_{\mathrm{e}}\right)$ de los tetrámeros, en $\mathrm{cm}^{-1}$. * significa que el modo es doblemente degenerado.

\begin{tabular}{c|c} 
Agregado & $\boldsymbol{\omega}_{\mathrm{e}}$ \\
\hline $\mathbf{( 6 )}$ & $83^{*}, 242^{*}, 373,643,1740$ \\
\hline $\mathbf{( 7 )}$ & $59,158,159,478,694,1101$ \\
& $147,181,309,532,538,1352^{67}$ \\
& $719,1004^{74}$ \\
\hline $\mathbf{( 8 )}$ & $43,185,297,321,384,2067$ \\
\hline $\mathbf{( 9 )}$ & $161,202,344,572,586,1440$ \\
& $558^{74}$ \\
& $194,228,303,505,509,1330^{70}$ \\
& $164,227,353,594,605,1276^{65}$ \\
\hline $\mathbf{( 1 0 )}$ & $50^{*}, 507,614 *, 1468,2277$ \\
& $39,489,560,1376,2220^{65}$ \\
\hline $\mathbf{( 1 1 )}$ & $86,228,276,339,848,2299$ \\
\hline $\mathbf{( 1 2 )}$ & $40,120,132,217,555,1094$ \\
\hline & $153^{*}, 210,430,756,757$ \\
& $149,187,417,740^{65}$
\end{tabular}

Respecto a los modos vibracionales, en frecuencias bajas se encontraron movimientos de tipo wagging y scissoring, en frecuencias intermedias estiramientos simétricos y asimétricos Al-Al y Al-N; y los estiramientos simétricos y asimétricos $\mathrm{N}-\mathrm{N}$ se encuentran a más de $1400 \mathrm{~cm}^{-1}$.

\section{Pentámeros}

Hemos encontrado diecinueve pentámeros, generados a partir de los tetrámeros anteriormente descriptos, de los cuales dieciséis resultaron de geometrías no lineales (Figuras 3.3 a 3.6). El agregado más estable es (15), caracterizado por un estado electrónico cuatriplete y una $\mathrm{E}_{\mathrm{at}}$ de 3.60 eV/át, de geometría plana no lineal. El único pentámero estudiado y encontrado también en la literatura (Tabla 3.5) es el confórmero (32), encontrándose muy buena correspondencia entre nuestros resultados y los reportados en la referencia 66. Los modos vibracionales de todos los confórmeros se muestran en la Tabla 3.6. 
(14)

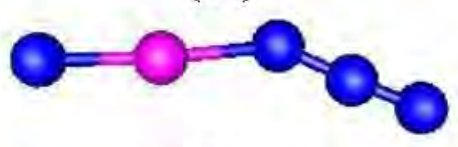

$\mathrm{r}_{\mathrm{N1} 1 \text {-Ai1 }}=1.74$

$\mathrm{r}_{\mathrm{A} 11-\mathrm{N} 2}=1.76$

$\mathrm{r}_{\mathrm{N} 2-\mathrm{N} 3}=1.21$

$\mathrm{r}_{\mathrm{N} 3-\mathrm{N} 4}=1.13$

$\mathrm{a}_{\mathrm{N} 1-\mathrm{A} 11-\mathrm{N} 2}=175.0$

$\mathrm{a}_{\mathrm{A} 11-\mathrm{N} 2 \mathrm{~N}-\mathrm{N} 3}=147.3$

$\mathrm{a}_{\mathrm{N} 2-\mathrm{N} 3-\mathrm{N} 4}=176.7$

(17)

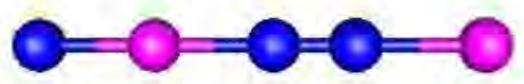

$\mathrm{r}_{\mathrm{N} 1-\mathrm{A} 11}=1.74$

$\mathrm{r}_{\mathrm{A} 11-\mathrm{N} 2}=1.76$

$\mathrm{r}_{\mathrm{N} 2 \mathrm{-N} 3}=1.21$

$\mathrm{r}_{\mathrm{N} 3-\mathrm{A} 12}=1.86$

(19)

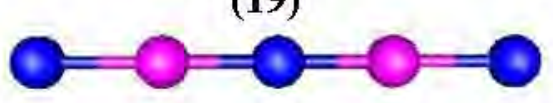

$\mathrm{r}_{\mathrm{N} 1-\mathrm{A} 11}=\mathrm{r}_{\mathrm{A} 12-\mathrm{N} 3}=1.80$

$\mathrm{r}_{\mathrm{A} 11-\mathrm{N} 2}=\mathrm{r}_{\mathrm{N} 2-\mathrm{A} 12}=1.69$
(15)

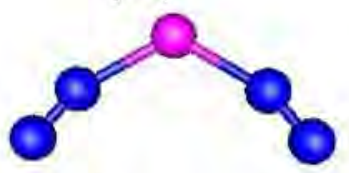

$\mathrm{r}_{\mathrm{N}-\mathrm{N}}=1.13$

$\mathrm{r}_{\mathrm{AL}-\mathrm{N}}=1.90$

$a_{\mathrm{N} 1-\mathrm{N} 2 \mathrm{-A11}}=\mathrm{a}_{\mathrm{A} 11-\mathrm{N} 3-\mathrm{N} 4}=160.6$

$\mathrm{a}_{\mathrm{N} 2-\mathrm{A} 11-\mathrm{N3} 3}=118.6$
(16)

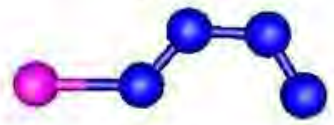

$\mathrm{r}_{\mathrm{A} 11-\mathrm{N} 1}=1.84$

$\mathrm{r}_{\mathrm{NN} 1-\mathrm{N} 2}=1.24$

$\mathrm{r}_{\mathrm{N}-\mathrm{N} 3}=1.35$

$\mathrm{r}_{\mathrm{N} 3-\mathrm{N} 4}=1.20$

$\mathrm{a}_{\mathrm{A} 11-\mathrm{N} 1-\mathrm{N} 2}=130.9$

$\mathrm{a}_{\mathrm{N} 1-\mathrm{N} 2-\mathrm{N} 3}=128.2$

$a_{\mathrm{N} 2-\mathrm{N} 3-\mathrm{N} 4}=123.3$

(18)

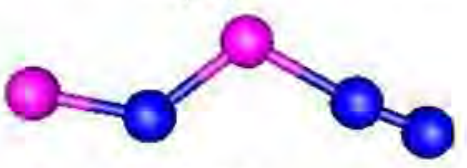

$\mathrm{r}_{\mathrm{A} 11-\mathrm{N} 1}=1.73$

$\mathrm{r}_{\mathrm{N1} 1-\mathrm{A} 12}=1.80$

$\mathrm{r}_{\mathrm{A} 12-\mathrm{N} 2}=1.86$

$\mathrm{r}_{\mathrm{N} 2-\mathrm{N} 3}=1.14$

$\mathrm{a}_{\mathrm{A} 11-\mathrm{N} 1-\mathrm{Al} 2}=131.0$

$\mathrm{a}_{\mathrm{N} 1-\mathrm{A} 12-\mathrm{N} 2}=112.8$

$\mathrm{a}_{\mathrm{A} 12-\mathrm{N} 2-\mathrm{N} 3}=173.3$

Figura 3.3. Geometrías de equilibrio de los pentámeros (14) a (19). Las distancias (r) están expresadas en $\AA$, los ángulos planos (a) y los diedros (d), en ${ }^{\circ}$. Los ángulos planos que no se especifican valen $180^{\circ}$ y los diedros $0.0^{\circ}$. 


\section{(20)}

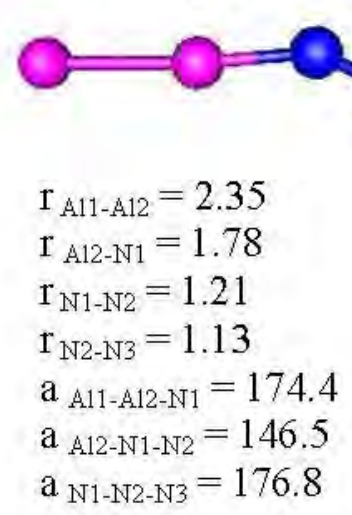

(21)

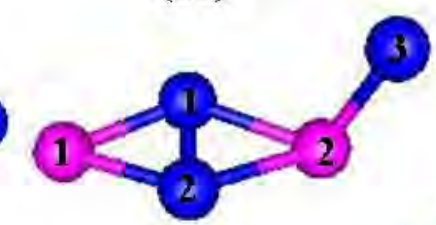

$\mathrm{r}_{\mathrm{A} 11-\mathrm{N} 1}=\mathrm{r}_{\mathrm{N} 2-\mathrm{A} 11}=1.80$

$\mathrm{r}_{\mathrm{N} 1-\mathrm{A} .22}=\mathrm{r}_{\mathrm{A} 12-\mathrm{N} 2}=1.91$

$\mathrm{r}_{\mathrm{A} 13-\mathrm{N} 3}=1.95$

$\mathrm{a}_{\mathrm{A} 11-\mathrm{N} 1-\mathrm{A} 12}=130.3$

$\mathrm{a}_{\mathrm{N} 1-\mathrm{A} 12-\mathrm{N} 2}=47.4$

$a_{\mathrm{N} 1-\mathrm{A} 12-\mathrm{N} 3}=108.2$

$\mathrm{d}_{\mathrm{A} 11-\mathrm{N} 2-\mathrm{A} 12-\mathrm{N} 3}=108.8$
(23)

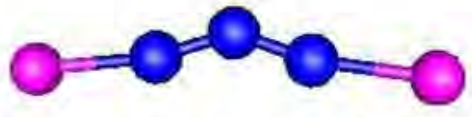

$\mathrm{r}_{\mathrm{A} 11-\mathrm{N} 1}=\mathrm{r}_{\mathrm{N} 3-\mathrm{A} 12}=1.83$

$\mathrm{r}_{\mathrm{N} 1-\mathrm{N} 2}=\mathrm{r}_{\mathrm{N} 2-\mathrm{N} 3}=1.21$

$\mathrm{a}_{\mathrm{A} 11-\mathrm{N} 1-\mathrm{N} 2}=\mathrm{a}_{\mathrm{N} 2-\mathrm{N} 3-\mathrm{A} 22}=168.4$

$\mathrm{a}_{\mathrm{N} 1-\mathrm{N} 2-\mathrm{N} 3}=143.3$

(22)

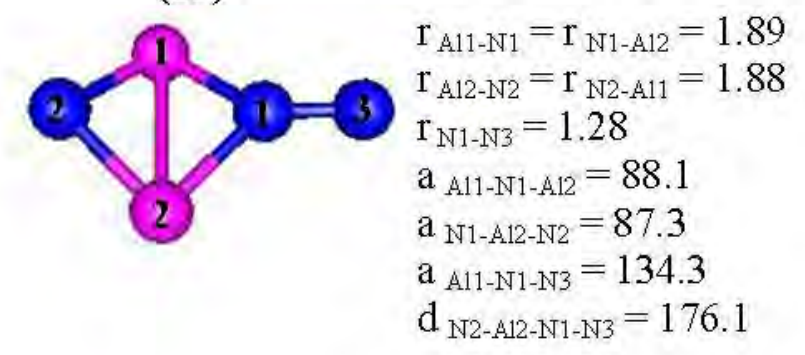

Figura 3.4. Geometrías de equilibrio de los pentámeros (20) a (23). Las distancias (r) están expresadas en $\AA$, los ángulos planos (a) y los diedros (d), en ${ }^{\circ}$. Los ángulos planos que no se especifican valen $180^{\circ}$ y los diedros $0.0^{\circ}$. 


\section{(24)}

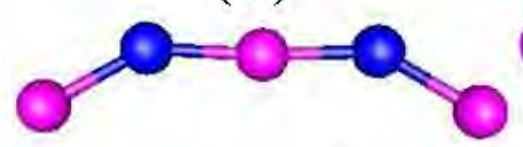

$$
\begin{aligned}
& \mathrm{r}_{\mathrm{A} 11-\mathrm{N} 1}=\mathrm{r}_{\mathrm{N} 2-\mathrm{A} 13}=1.72 \\
& \mathrm{r}_{\mathrm{N} 1-\mathrm{A} 12}=\mathrm{r}_{\mathrm{A} 12-\mathrm{N} 2}=1.71 \\
& \mathrm{a}_{\mathrm{A} 1-\mathrm{N}-\mathrm{A} 11} \approx 146.5 \\
& \mathrm{a}_{\mathrm{N} 1-\mathrm{A} 12-\mathrm{N} 2}=175.0
\end{aligned}
$$

(26)

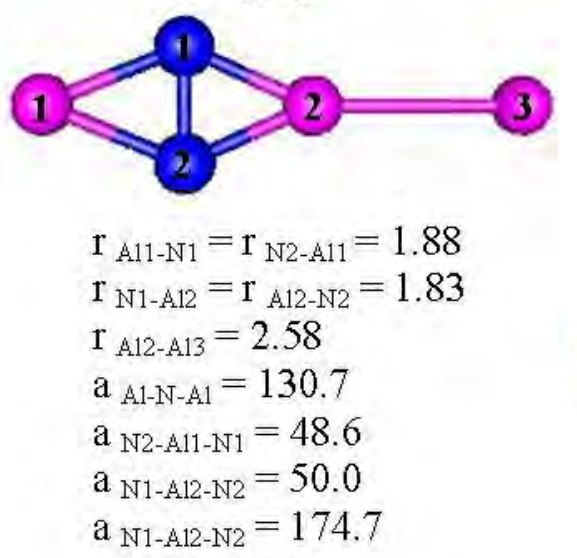

(25)

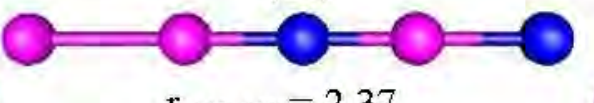

$\mathrm{r}_{\mathrm{A} 11-\mathrm{A} 12}=2.37$

$\mathrm{r}_{\mathrm{A} 12-\mathrm{N} 1}=1.76$

$\mathrm{r}_{\mathrm{N} 1-\mathrm{A}_{13} 3}=1.66$

$\mathrm{r}_{\mathrm{A} 13-\mathrm{N} 2}=1.86$

$\mathrm{r}_{\mathrm{A} 11-\mathrm{N} 1}=\mathrm{r}_{\mathrm{A} 11-\mathrm{N} 2}=\mathrm{r}_{\mathrm{N} 2-\mathrm{A} 13}=1.89$

$\mathrm{r}_{\mathrm{A} 12-\mathrm{N} 2}=2.03$

$\mathrm{a}_{\mathrm{A} 1 \mathrm{~N}-\mathrm{N}-\mathrm{A} 1}=100.0$

$\mathrm{d}_{\mathrm{N} 1-\mathrm{A} 12-\mathrm{A} 11-\mathrm{A} 13}=47.9$
(28)

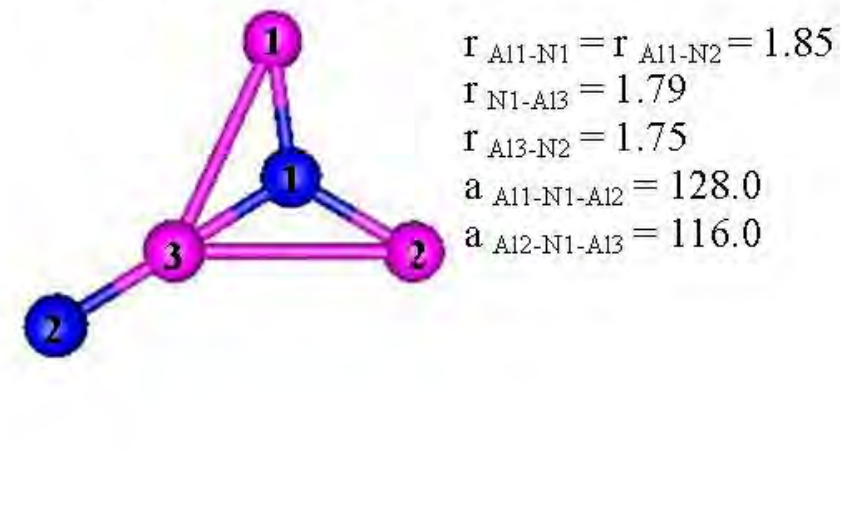

Figura 3.5. Geometrías de equilibrio de los pentámeros (24) a (28). Las distancias (r) están expresadas en $\AA$, los ángulos planos (a) y los diedros (d), en ${ }^{\circ}$. Los ángulos planos que no se especifican valen $180^{\circ} \mathrm{y}$ los diedros $0.0^{\circ}$. 
(29)

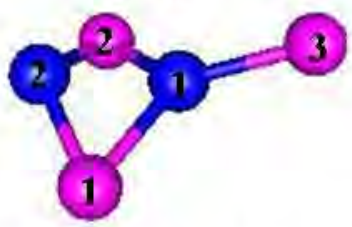

$\mathrm{r}_{\mathrm{A} 11-\mathrm{N} 2}=\mathrm{r}_{\mathrm{A} 12-\mathrm{N} 2}=\mathrm{r}_{\mathrm{A} 13-\mathrm{N} 1}=1.81$

$\mathrm{r}_{\mathrm{A} 12-\mathrm{N} 1}=_{\text {A11-N1 }}=1.94$

$\mathrm{a}_{\mathrm{A} 11-\mathrm{N} 1-\mathrm{A} .12}=108.1$

$\mathrm{a}_{\mathrm{A} 11-\mathrm{N} 2-\mathrm{A} .12}=119.6$

$\mathrm{a}_{\mathrm{A} 11-\mathrm{N} 1-\mathrm{A} 13}=125.9$

$\mathrm{d}_{\mathrm{N} 2-\mathrm{A} 11-\mathrm{N} 1-\mathrm{A} 13}=154.5$

(31)

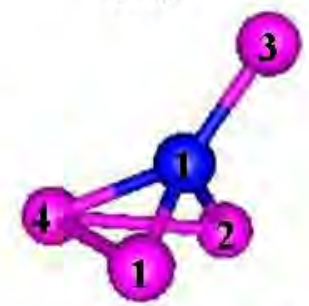

$\mathrm{r}_{\text {A11-N1 }}=\mathrm{r}_{\text {A12-N1 }}=1.97$

$\mathrm{r}_{\mathrm{N} 1-\mathrm{A} 13}=1.86$

$\mathrm{r}_{\mathrm{N} 1-\mathrm{A} .14}=2.02$

$\mathrm{a}_{\mathrm{A} 11-\mathrm{N} 1-\mathrm{A} 12}=113.4$

$\mathrm{a}_{\mathrm{A} 11-\mathrm{N} 1-\mathrm{A} 13}=115.9$

$\mathrm{a}_{\mathrm{A} 11-\mathrm{N} 1-\mathrm{A} 14}=79.0$

$\mathrm{d}_{\mathrm{A} 11-\mathrm{N} 1-\mathrm{A} 12-\mathrm{A} 14}=72.6$
(30)

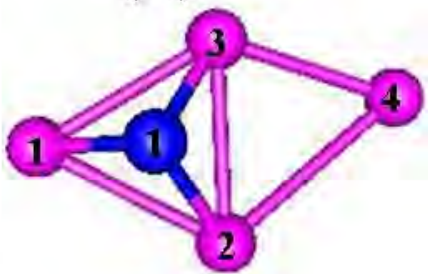

$\mathrm{r}_{\mathrm{A} 11-\mathrm{N} 1}=\mathrm{r}_{\mathrm{A} 12-\mathrm{N} 1}=\mathrm{r}_{\mathrm{A} 13-\mathrm{N} 1}=1.90$

$\mathrm{r}_{\mathrm{A} 12-\mathrm{A} 13}=2.64$

$\mathrm{r}_{\mathrm{A} 13-\mathrm{A} 14}=2.55$

$\mathrm{a}_{\mathrm{A} 11-\mathrm{N} 1-\mathrm{A} .2}=90.5$

$\mathrm{a}_{\mathrm{A} .11-\mathrm{N} 1-\mathrm{A}_{13}}=89.4$

$\mathrm{a}_{\mathrm{A} .12-\mathrm{N} 1-\mathrm{A}_{13}}=86.9$

$\mathrm{a}_{\mathrm{A} 12-\mathrm{A} 13-\mathrm{A} 14}=71.2$

$\mathrm{d}_{\mathrm{A} 11-\mathrm{A} 12-\mathrm{A} 13-\mathrm{N} 1}=53.9$

(32)

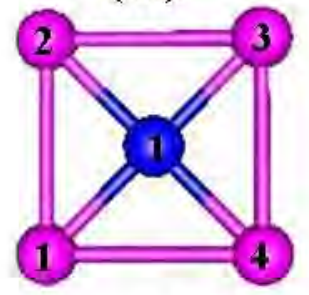

$$
\begin{aligned}
& \mathrm{r}_{\mathrm{A} 1 \mathrm{-A} 1 \mathrm{1}}=2.77 \\
& \mathrm{r}_{\mathrm{N}-\mathrm{A} 1}=1.96 \\
& \mathrm{a}_{\mathrm{A} 1-\mathrm{A} 1-\mathrm{A} 1}=90.0
\end{aligned}
$$

Figura 3.6. Geometrías de equilibrio de los pentámeros (28) a (32). Las distancias (r) están expresadas en $\AA$, los ángulos planos (a) y los diedros (d), en ${ }^{\circ}$. Los ángulos planos que no se especifican valen $180^{\circ} \mathrm{y}$ los diedros $0.0^{\circ}$.

La distancia Al-N se encuentra, en estos casos, entre 1.68 y $2.03 \AA$, la distancia de enlace Al-Al entre 2.46 y $2.81 \AA$, y la distancia N-N entre 1.10 y $1.20 \AA$, característico de enlaces dobles y triples. 
Tabla 3.5. Multiplicidades de espín $\left(M_{S}\right)$ y energías de atomización $\left(E_{a t}\right)$ en eV/át, de los pentámeros.

\begin{tabular}{c|c|c} 
Agregado & $\mathbf{M}_{\mathbf{s}}$ & $\mathbf{E}_{\mathbf{a t}}$ \\
\hline $\mathbf{( 1 4 )}$ & 2 & 3.25 \\
\hline $\mathbf{( 1 5 )}$ & 4 & 3.60 \\
\hline $\mathbf{( 1 6 )}$ & 6 & 2.48 \\
\hline $\mathbf{( 1 7 )}$ & 2 & 2.68 \\
\hline $\mathbf{( 1 8 )}$ & 4 & 3.10 \\
\hline $\mathbf{( 1 9 )}$ & 4 & 2.36 \\
\hline $\mathbf{( 2 0 )}$ & 4 & 2.83 \\
\hline $\mathbf{( 2 1 )}$ & 6 & 2.30 \\
\hline $\mathbf{( 2 2 )}$ & 6 & 2.58 \\
\hline $\mathbf{( 2 3 )}$ & 2 & 3.15 \\
\hline $\mathbf{( 2 4 )}$ & 2 & 2.93 \\
\hline $\mathbf{( 2 5 )}$ & 6 & 1.84 \\
\hline $\mathbf{( 2 6 )}$ & 4 & 2.32 \\
\hline $\mathbf{( 2 7 )}$ & 2 & 2.72 \\
\hline $\mathbf{( 2 8 )}$ & 2 & 2.68 \\
\hline $\mathbf{( 2 9 )}$ & 2 & 2.69 \\
\hline $\mathbf{( 3 0 )}$ & 8 & 1.26 \\
\hline $\mathbf{( 3 1 )}$ & 4 & 2.15 \\
\hline $\mathbf{( 3 2 )}$ & 2 & 2.54 \\
& $2^{66}$ & $2.62^{66}$
\end{tabular}

Se observa, por la Tabla 3.5 que el orden ascendente en la energía de atomización de los pentámeros es el siguiente: (30) $<$ (25) $<$ (31) $<$ (21) $<$ (26) $<$ (19) $<$ (16) $<$ (32) $<$ (22) $<$ (17) $<$ (28) $<$ (29) $<$ (27) $<$ (20) $<$ (24) $<$ (18) $<$ (23) $<$ (14) $<$ (15) . 
Tabla 3.6. Modos normales de vibración $\left(\omega_{\mathrm{e}}\right)$ de los pentámeros, en $\mathrm{cm}^{-1}$. $*$ significa que el modo es doblemente degenerado.

\begin{tabular}{c|c} 
Agregado & $\boldsymbol{\omega}_{\mathbf{e}}$ \\
\hline $\mathbf{( 1 4 )}$ & $71,130,160,518,594,609,942,1470,2309$ \\
\hline $\mathbf{( 1 5 )}$ & $40,134,183,345,369,381,448,1769,2034$ \\
\hline $\mathbf{( 1 6 )}$ & $76,122,278,429,583,783,900,1486,1515$ \\
\hline $\mathbf{( 1 7 )}$ & $53,60,110,122,178,219,359,662,952,1776$ \\
\hline $\mathbf{( 1 8 )}$ & $62,71,145,276,303,497,583,996,1932$ \\
\hline $\mathbf{( 1 9 )}$ & $36,46,157,159,182,186,430,701,881,1166$ \\
\hline $\mathbf{( 2 0 )}$ & $53,93,124,340,594,600,661,1459,2288$ \\
\hline $\mathbf{( 2 1 )}$ & $85,146,193,322,448,546,747,775,873$ \\
\hline $\mathbf{( 2 2 )}$ & $181,204,274,343,525,536,552,641,1169$ \\
\hline $\mathbf{( 2 3 )}$ & $54,80,96,119,345,584,597,1407,1788$ \\
\hline $\mathbf{( 2 4 )}$ & $43,79,103,202,209,446,688,1088,1124$ \\
\hline $\mathbf{( 2 5 )}$ & $25 *, 11 *, 131 *, 301,539,705,1245$ \\
\hline $\mathbf{( 2 6 )}$ & $55,60,161,232,331,458,718,726,812$ \\
\hline $\mathbf{( 2 7})$ & $174,184,223,317,410,464,480,551,728$ \\
\hline $\mathbf{( 2 8 )}$ & $72,104,132,184,228,407,741,754,978$ \\
\hline $\mathbf{2 9})$ & $62,75,154,314,351,390,605,756,850$ \\
\hline $\mathbf{( 3 0 )}$ & $45,100,206,245,283,337,532,550,566$ \\
\hline $\mathbf{( 3 1 )}$ & $80,82,159,167,181,308,386,390,697$ \\
\hline $\mathbf{( 3 2})$ & $49,109,110,217,218,226,380,525,527$
\end{tabular}

Los pentámeros presentan modos vibracionales de tipo wagging, scissoring, twisting y rocking a frecuencias bajas, estiramientos asimétricos y simétricos Al-Al y Al-N en frecuencias intermedias, y en frecuencias mayores a $1200 \mathrm{~cm}^{-1}$ se ven estiramientos simétricos y asimétricos N-N.

Análisis de cargas derivadas del potencial electrostático y de la densidad de espín Los análisis de cargas derivadas de potenciales electrostáticos (ESP) y de la densidad de espín, se realizaron al mismo nivel de teoría antes descripto. El cálculo de la densidad de espín se realizó mediante la obtención de la configuración electrónica natural. En las Tablas 3.7 a 3.9 y Figuras 3.7 a 3.10 se muestran los resultados obtenidos.

Tabla 3.7. Análisis de cargas ESP (en unidades de $|e|$ ) y densidades de espín, entre paréntesis y en u.a., del dímero y los trímeros. Los átomos se numeran de izquierda a derecha.

\begin{tabular}{c|l|l|l|l} 
Agregado & Al1 & Al2 & N1 & N2 \\
\hline $\mathbf{( 1 )}$ & $0.42(0.28)$ & & $-0.42(1.71)$ & \\
\hline $\mathbf{( 2 )}$ & $0.05(0.45)$ & & $0.26(0.15)$ & $-0.32(0.40)$ \\
\hline $\mathbf{( 3 )}$ & $1.06(-0.19)$ & & $-0.53(1.60)$ & $-0.53(1.60)$ \\
\hline $\mathbf{( 4 )}$ & $-0.05(1.70)$ & $0.32(1.15)$ & $-0.27(2.15)$ & \\
\hline $\mathbf{( 5 )}$ & $0.28(0.36)$ & $0.27(0.36)$ & $-0.55(0.26)$ &
\end{tabular}


Tabla 3.8. Análisis de cargas ESP (en unidades de |e|) y densidades de espín, entre paréntesis y en u.a., de los tetrámeros. Los átomos se numeran de izquierda a derecha, o como se muestra en

\begin{tabular}{c|l|l|l|l|l|l}
$\#$ & Al1 & Al2 & Al3 & N1 & N2 & N3 \\
\hline $\mathbf{( 6 )}$ & $0.04(0.22)$ & $0.04(0.22)$ & & $-0.04(0.78)$ & $-0.04(0.78)$ & \\
\hline $\mathbf{( 7 )}$ & $0.20(0.06)$ & $1.01(-0.11)$ & & $-0.77(0.23)$ & $-0.45(1.85)$ & \\
\hline $\mathbf{( 8 )}$ & $0.003(1.84)$ & $0.12(1.69)$ & & $0.06(0.09)$ & $-0.19(0.39)$ & \\
\hline $\mathbf{( 9 )}$ & 0.04 & 0.04 & & -0.06 & -0.02 & \\
\hline $\mathbf{( 1 0 )}$ & 0.11 & & & -0.03 & 0.23 & -0.32 \\
\hline $\mathbf{( 1 1 )}$ & 0.40 & & & -0.57 & 0.31 & -0.13 \\
\hline $\mathbf{( 1 2 )}$ & $-0.04(1.08)$ & $0.54(0.43)$ & $0.28(0.31)$ & $-0.78(0.20)$ & & \\
\hline $\mathbf{( 1 3 )}$ & -0.19 & -0.17 & -0.19 & 0.56 & &
\end{tabular}


Tabla 3.9. Análisis de cargas ESP (en unidades de |e|) y densidades de espín, entre paréntesis y en u.a., de los pentámeros. Los átomos se numeran de izquierda a derecha o como muestran las Figuras 3.3 a 3.6.

\begin{tabular}{c|l|l|l|l|l|l|l|l}
$\#$ & Al1 & Al2 & Al3 & Al4 & N1 & N2 & N3 & N4 \\
\hline $\mathbf{( 1 4 )}$ & $1.00(-0.02)$ & & & & $-0.68(1.03)$ & $-0.55(0.00)$ & $0.54(0.00)$ & $-0.31(0.01)$ \\
\hline $\mathbf{( 1 5 )}$ & $0.21(1.71)$ & & & & $-0.17(0.51)$ & $0.07(0.15)$ & $0.07(0.15)$ & $-0.17(0.51)$ \\
\hline $\mathbf{( 1 6 )}$ & $0.30(1.89)$ & & & $-0.07(1.02)$ & $-0.03(0.79)$ & $0.02(0.13)$ & $-0.21(1.19)$ \\
\hline $\mathbf{( 1 7 )}$ & $1.13(0.18)$ & $0.06(0.19)$ & & $-0.78(-0.96)$ & $-0.85(0.73)$ & $0.45(0.87)$ & \\
\hline $\mathbf{( 1 8 )}$ & $0.44(0.53)$ & $0.16(1.09)$ & & $-0.48(0.63)$ & $0.20(0.20)$ & $-0.33(0.54)$ & \\
\hline $\mathbf{( 1 9 )}$ & $1.31(-0.11)$ & $1.31(-0.11)$ & & & $-0.61(1.54)$ & $-1.40(0.16)$ & $-0.61(1.54)$ & \\
\hline $\mathbf{( 2 0}$ & $0.04(1.81)$ & $0.21(1.11)$ & & & $-0.41(0.03)$ & $0.64(0.00)$ & $-0.47(0.06)$ & \\
\hline $\mathbf{( 2 1})$ & $0.62(0.81)$ & $0.33(0.76)$ & & & $-0.34(0.61)$ & $-0.34(0.62)$ & $-0.26(2.21)$ & \\
\hline $\mathbf{( 2 2})$ & $0.44(0.94)$ & $0.44(0.94)$ & & & $0.25(0.22)$ & $-0.55(1.30)$ & $-0.57(1.56)$ & \\
\hline $\mathbf{( 2 3 )}$ & $0.05(0.10)$ & $0.05(0.10)$ & & & $0.10(0.20)$ & $-0.30(0.36$ & $0.10(0.22)$ & \\
\hline $\mathbf{( 2 4 )}$ & $0.32(0.35)$ & $0.93(-0.06)$ & $0.29(0.34)$ & & $-0.79(0.17)$ & $-0.75(0.17)$ & & \\
\hline $\mathbf{( 2 5 )}$ & $-0.10(1.92)$ & $0.34(0.97)$ & $1.30(-0.05)$ & & $-1.06(0.26)$ & $-0.48(1.91)$ & & \\
\hline $\mathbf{( 2 6 )}$ & $0.32(0.28)$ & $0.56(0.47)$ & $0.01(1.19)$ & & $-0.44(0.58)$ & $-0.44(0.58)$ & & \\
\hline $\mathbf{( 2 7})$ & $0.27(0.45)$ & $0.004(-0.01)$ & $0.28(0.45)$ & & $-0.28(0.06)$ & $-0.27(0.06)$ & & \\
\hline $\mathbf{( 2 8 )}$ & $-0.01(0.00)$ & $-0.01(0.00)$ & $0.72(-0.03)$ & & $0.07(0.00)$ & $-0.78(1.01)$ & & \\
\hline $\mathbf{( 2 9})$ & $0.11(0.43)$ & $0.08(0.06)$ & $-0.10(0.08)$ & & $0.14(-0.01)$ & $-0.23(0.43)$ & & \\
\hline $\mathbf{( 3 0 )}$ & $0.08(1.51)$ & $0.19(1.49)$ & $0.20(1.35)$ & $-0.10(1.71)$ & $-0.38(0.92)$ & & & \\
\hline $\mathbf{( 3 1 )}$ & $-0.20(0.65)$ & $-0.20(0.65)$ & $-0.31(0.07)$ & $-0.21(1.44)$ & $0.93(0.17)$ & & & \\
\hline $\mathbf{( 3 2 )}$ & $-0.18(0.24)$ & $-0.20(0.25)$ & $-0.18(0.25)$ & $-0.18(0.25)$ & $0.74(-0.01)$ & & & \\
\hline
\end{tabular}




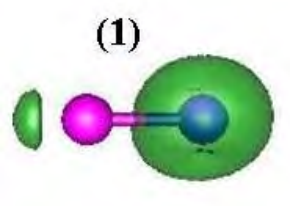

(2)

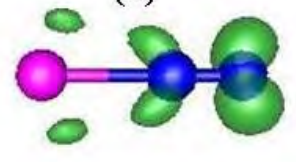

(3)

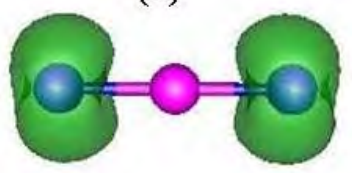

(4)

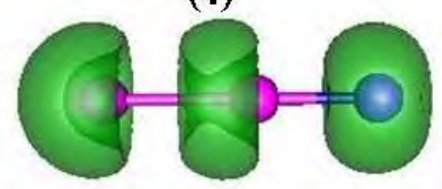

(5)

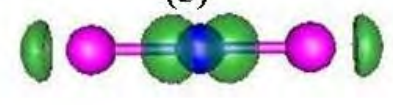

Figura 3.7. Densidades de espín del dímero y los trímeros. Las superficies se graficaron con un valor de isosuperficie de 0.015 u.a.

(6)

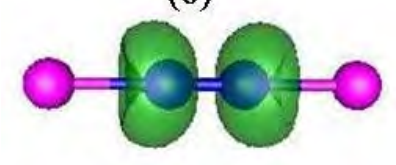

(12)

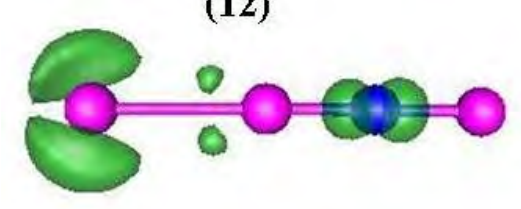

(7)

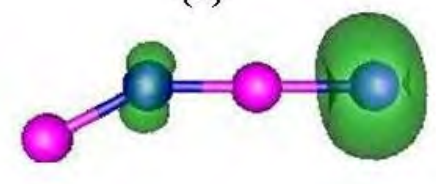

Figura 3.8. Densidades de espín de los tetrámeros de capa abierta. Las superficies se graficaron con un valor de isosuperficie de 0.015 u.a. 
(14)

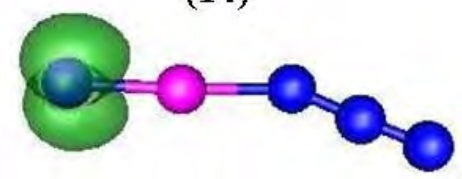

(17)

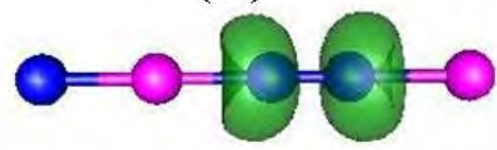

(19)

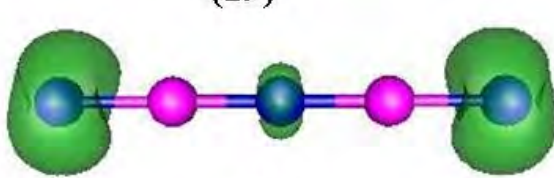

(20)

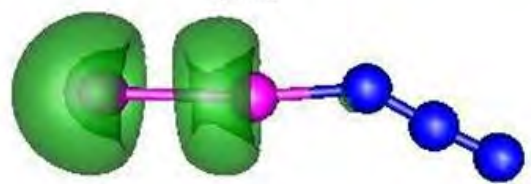

(15)

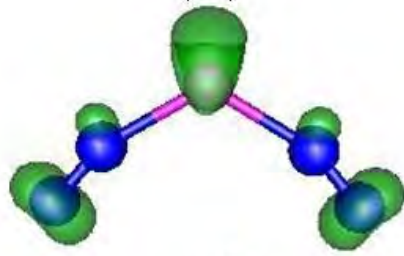

(18)

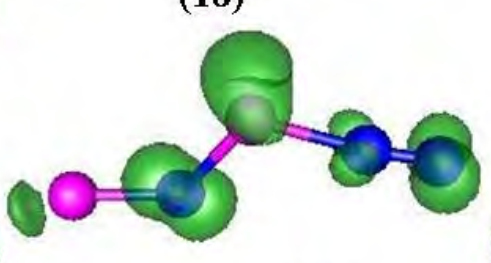

(22)

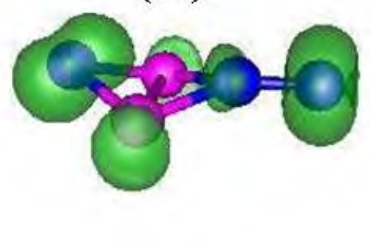

(16)

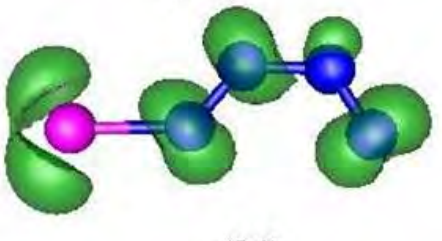

(21)

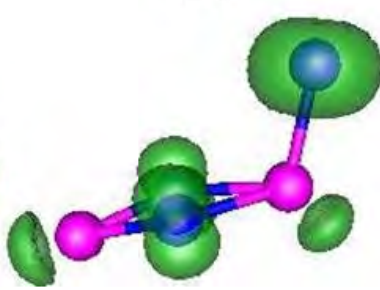

Figura 3.9. Densidades de espín de pentámeros (14) a (22). Las superficies se graficaron con un valor de isosuperficie de 0.015 u.a.

54 
(23)

(24)
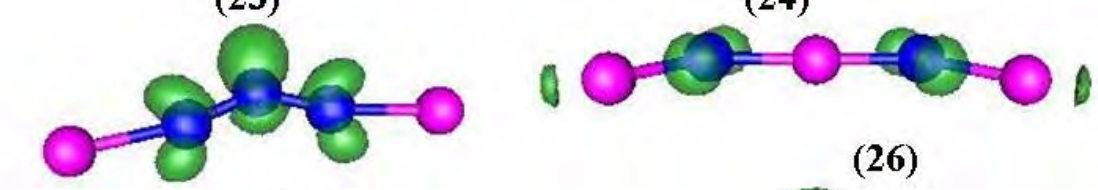

(26)

(25)

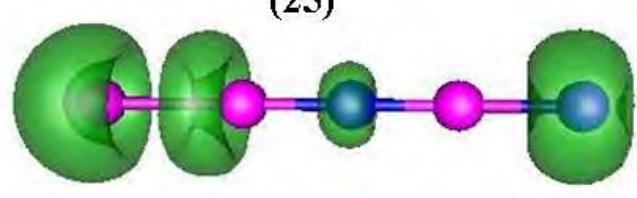

(27)

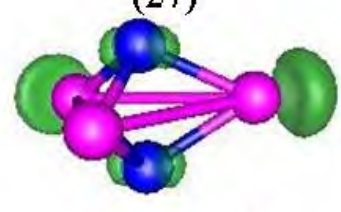

(30)

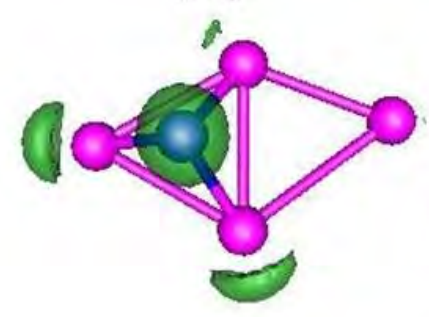

(28)

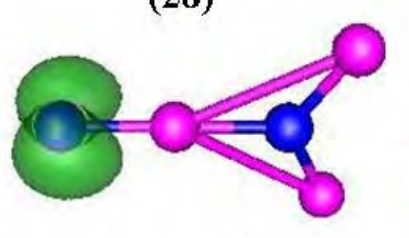

(31)

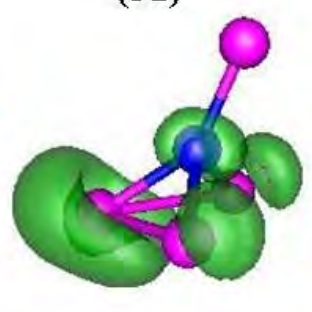

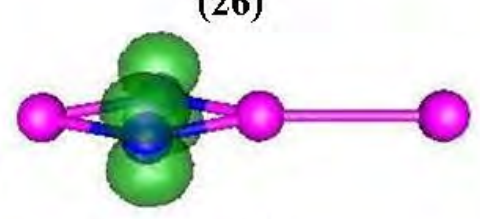

(29)

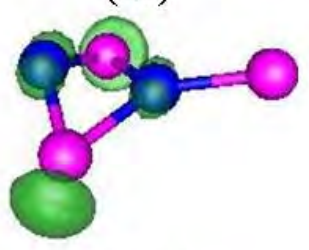

(32)

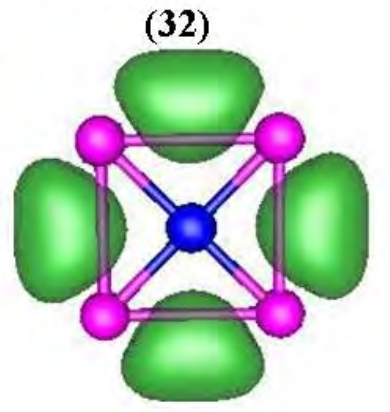

Figura 3.10. Densidades de espín de pentámeros (23) a (32). Las superficies se graficaron con un valor de isosuperficie de 0.015 u.a.

\subsection{Potenciales electrostáticos moleculares}

En las Figuras 3.11 a 3.14 se muestran los gráficos de potenciales electrostáticos moleculares (MEP) calculados para todos los sistemas. El color azul indica una zona susceptible de ataque electrofílico, mientras que el color rojo muestra una zona probable de ataque nucleofílico, los colores verdes y amarillos son zonas intermedias de valores negativos y positivos muy bajos, respectivamente.

Asimismo, en la Figura 3.11 se muestran los gráficos de MEP correspondientes al dímero y los trímeros junto con los valores de cargas ESP obtenidas para cada átomo, con el objeto de mostrar la buena concordancia entre ellos, Tablas 3.7 a 3.9. Por ello se evidencia que las cargas ESP son, por sí solas, un buen indicativo de la naturaleza de la zona susceptible de ataque, ya sea nucleofílico o electrofílico. 
(1)

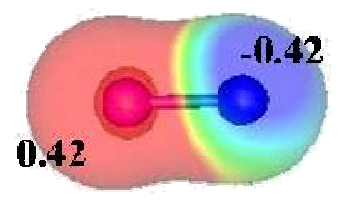

(5)

(2)

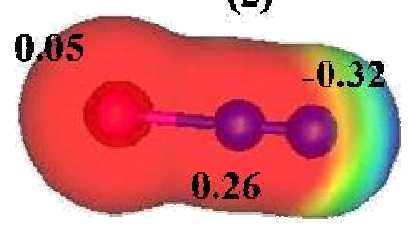

(4)

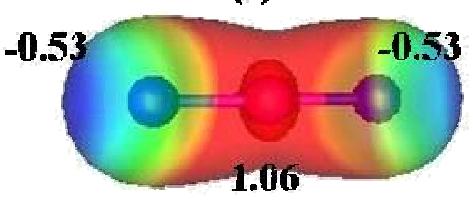

(1)
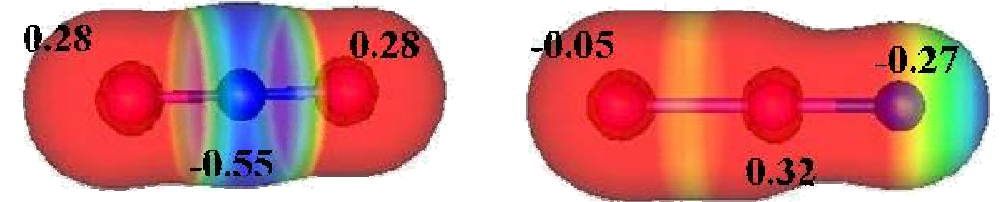

Figura 3.11. Superficies de potenciales electrostáticos moleculares del dímero y los trímeros. También se muestran las cargas ESP calculadas. Las superficies se graficaron con un valor de isosuperficie de la densidad electrónica de 0.01 u.a.

En el dímero la zona de ataque electrofílico se localiza en el átomo de nitrógeno. A medida que el sistema crece la tendencia se mantiene, en general en los trímeros la zona de MEP negativo está en los alrededores de átomos de nitrógeno y la zona de MEP positiva en los átomos de aluminio, con excepción del átomo N1 en (2). Comparando con lo mostrado en la Figura 3.7 y la Tabla 3.7, se ve que las zonas de MEP negativo son zonas donde se encuentran electrones desapareados.

(6)

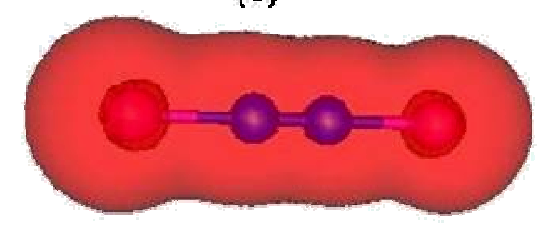

(9)

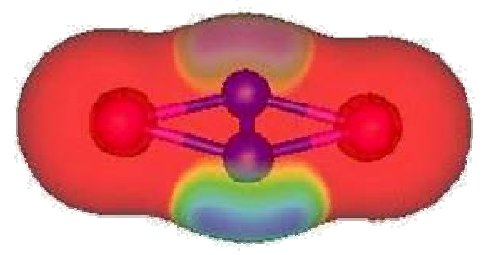

(12)

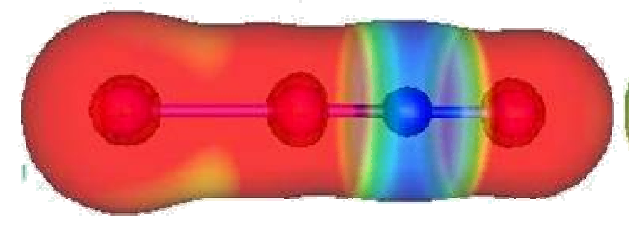

(7)

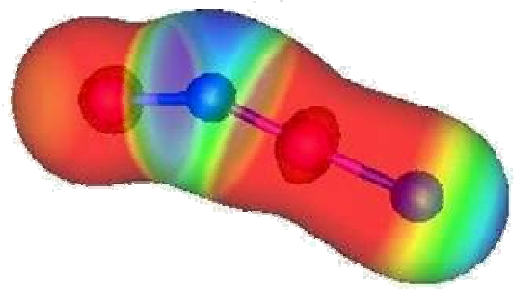

(10)

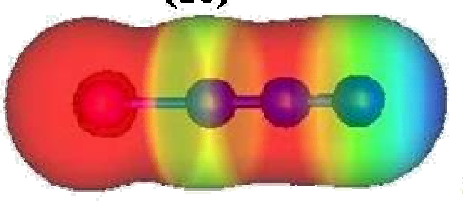

(13)
(8)

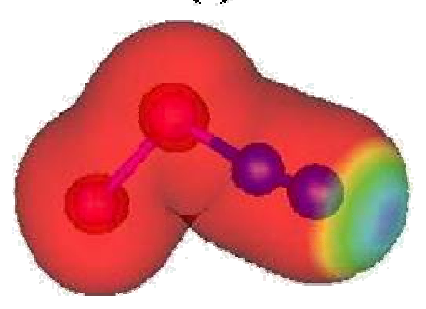

(11)
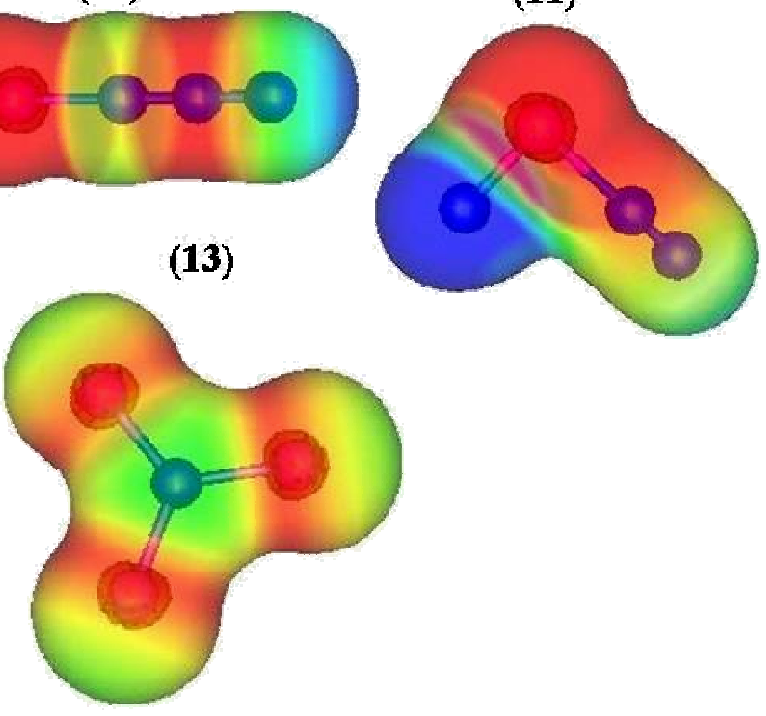

Figura 3.12. Superficies de potenciales electrostáticos moleculares para los tetrámeros. Las superficies se graficaron con un valor de isosuperficie de la densidad electrónica de 0.01 u.a. 
En los tetrámeros la tendencia se mantiene, los átomos de nitrógeno de los extremos localizan las zonas susceptibles de ataque electrofílico, con excepción del confórmero (6), cuya superficie es enteramente susceptible de ataque nucleofílico, a pesar de que posee cargas ESP negativas muy bajas en los nitrógenos (Tabla 3.8); y el confórmero (13), que distribuye las zonas de MEP negativo en los átomos de aluminio que rodean al átomo de nitrógeno, de MEP positivo. A diferencia de lo encontrado para los trímeros, en las zonas de MEP negativo de los tetrámeros no siempre se observan electrones desapareados.

\section{(14)}

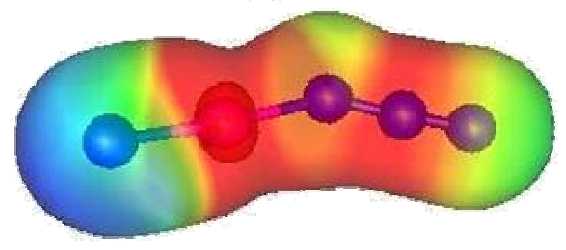

(16)

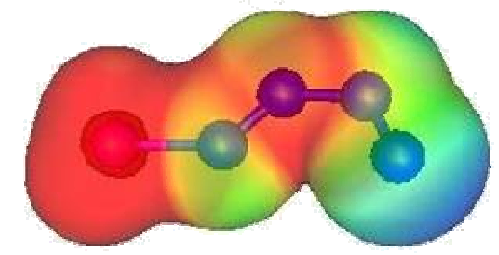

(18)

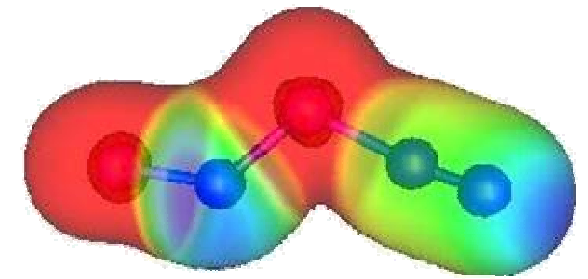

(20)

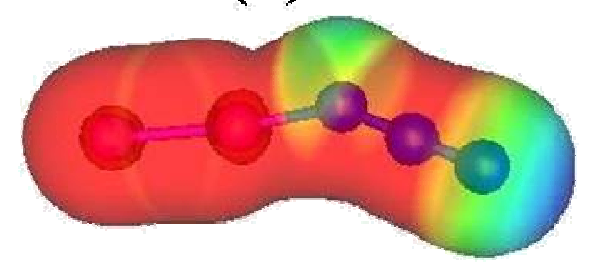

(22)

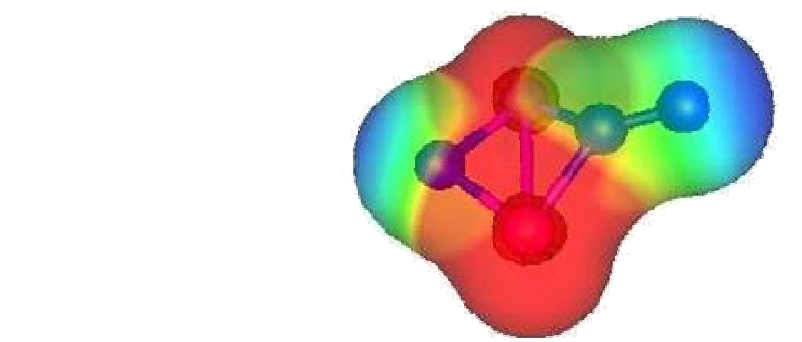

Figura 3.13. Superficies de potenciales electrostáticos moleculares para los pentámeros (14) a (22). Las superficies se graficaron con un valor de isosuperficie de la densidad electrónica de

0.01 u.a.

(15)

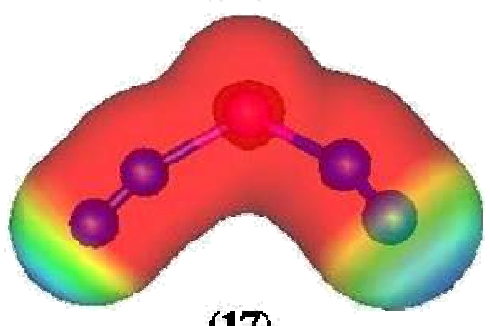

(17)

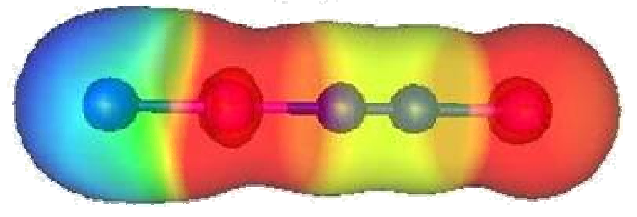

(19)

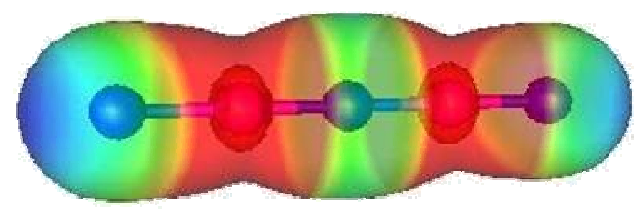

(21)

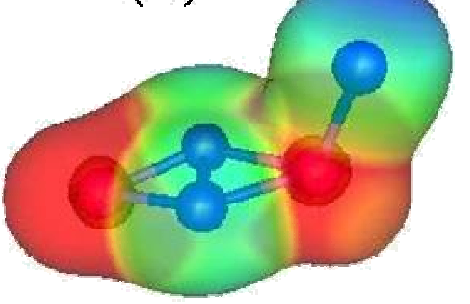

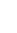

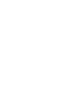


(23)

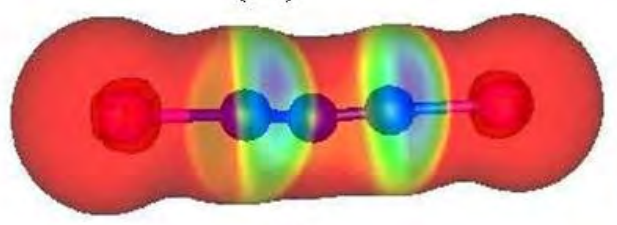

(25)

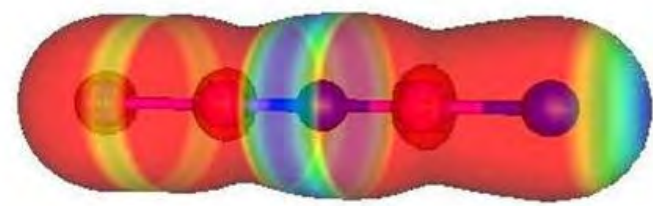

(27)

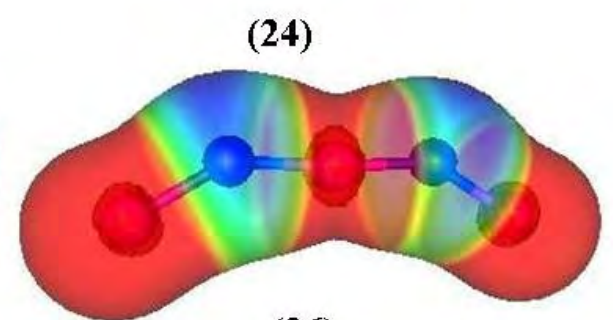

(26)

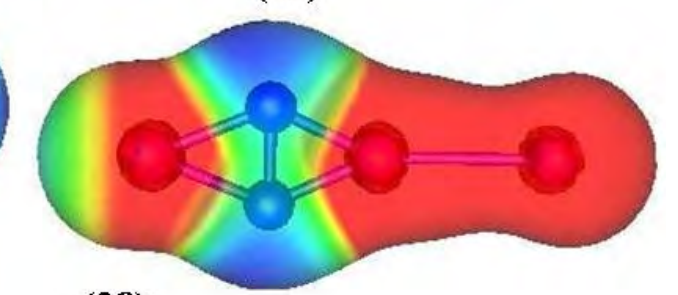

(28)

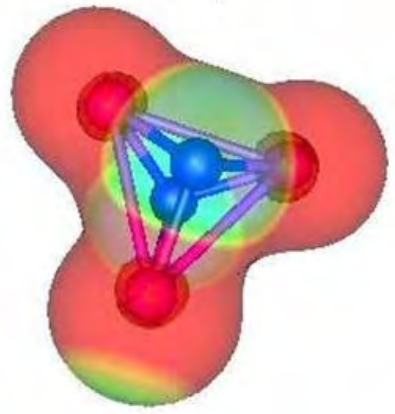

(29)
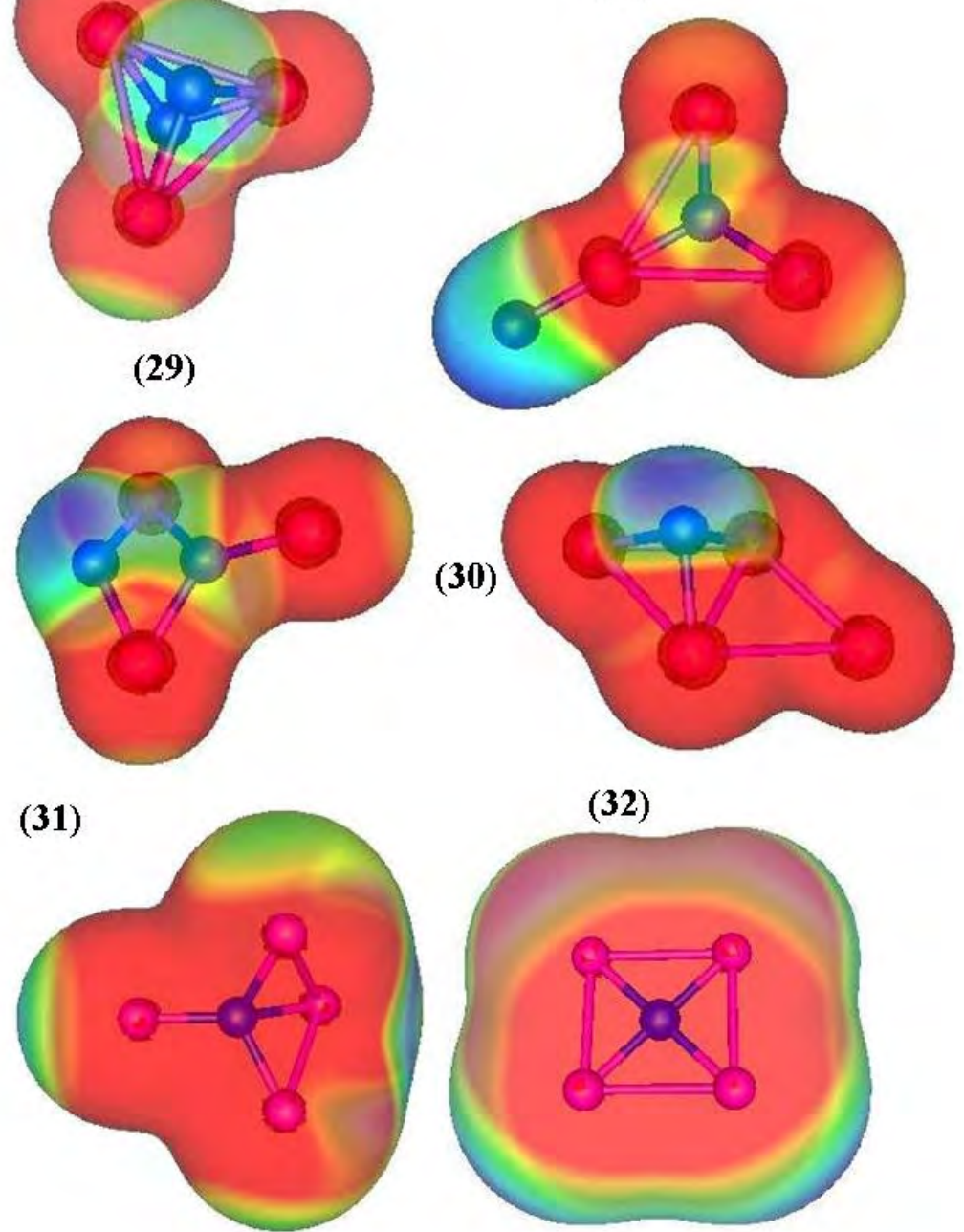

(32)

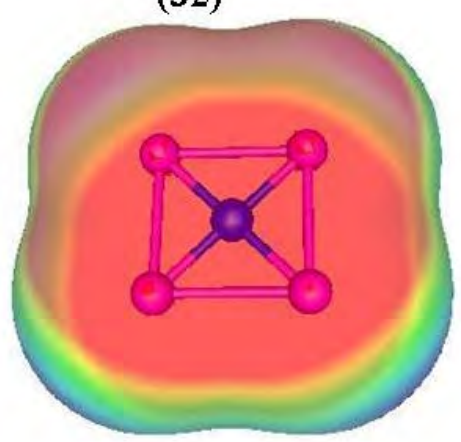

Figura 3.14. Superficies de potenciales electrostáticos moleculares para los pentámeros (23) a (32). Las superficies se graficaron con un valor de isosuperficie de la densidad electrónica de 0.01 u.a., con excepción de (31) y (32), en los que se usó un valor de 0.002 u.a. y 0.005 u.a., respectivamente. 
Una total concordancia con los resultados anteriores se encuentra en los pentámeros, que ubican las zonas susceptibles de ataque electrofílico en los alrededores de átomos de nitrógeno. En particular, aquellos agregados con varios átomos de nitrógeno unidos entre sí, (14), (15), (16) y (18), concentran la zona de MEP negativo en los átomos de nitrógeno de los extremos. Los agregados (31) y (32) son la excepción, ubicándose la zona susceptible de ataque electrofílico en la zona de los átomos de aluminio, que rodean al único átomo de nitrógeno, de MEP positivo. Estos dos agregados también poseen electrones desapareados en las zonas de MEP negativo. Correlacionando los datos de la Tabla 3.9 con los de las Figuras $3.9,3.10,3.13$ y 3.14 , se observa en el resto de los pentámeros que no siempre se ubican electrones desapareados en zonas de MEP negativo, en acuerdo con lo encontrado para los trímeros (Figuras 3.7 y 3.11), y a diferencia de lo obtenido para los tetrámeros (Figuras 3.8 y $3.12)$.

\subsection{Patrones de crecimiento}

En las Figuras 3.15 a 3.17 se muestran los patrones de crecimiento obtenidos. Estos patrones se construyen a partir del dímero (1) agregando átomos de $\mathrm{N}$ y de $\mathrm{Al}$, siguiendo el camino de aumento de la energía de atomización. En algunos casos los agregados crecen sólo hasta cuatro átomos. Se propone el patrón de crecimiento más probable para agregados binarios constituidos por $\mathrm{Al}$ y N.

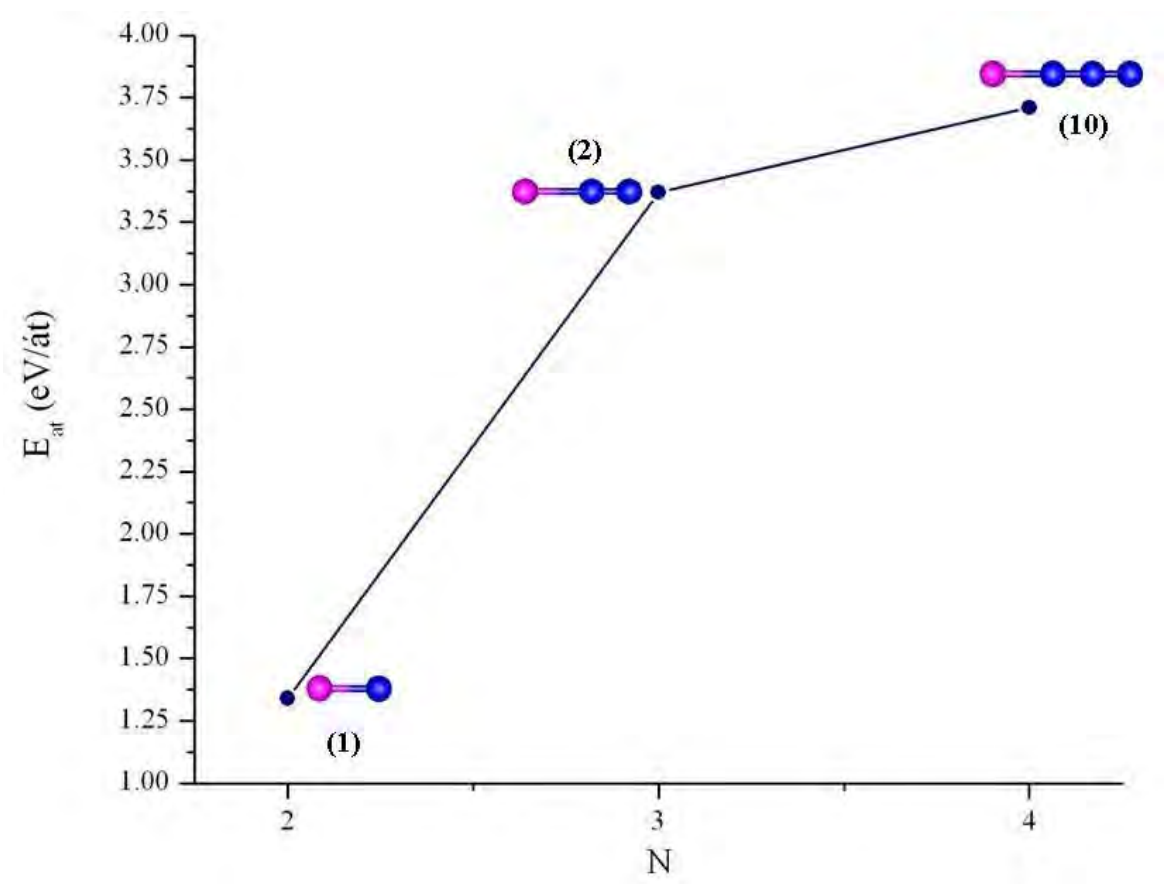

Figura 3.15. Patrón de crecimiento del trímero (2).

Se observa en la Figura 3.15 que el patrón de crecimiento obtenido a partir del trímero (2) termina en el tetrámero, dado que no se encontró un pentámero cuya energía de atomización sea mayor a la de (10).

En las Figuras 3.16.a, 3.16.b y 3.16.c se ven tres posibilidades de patrones de crecimiento a partir del confórmero (3), obteniéndose tres tetrámeros distintos. 


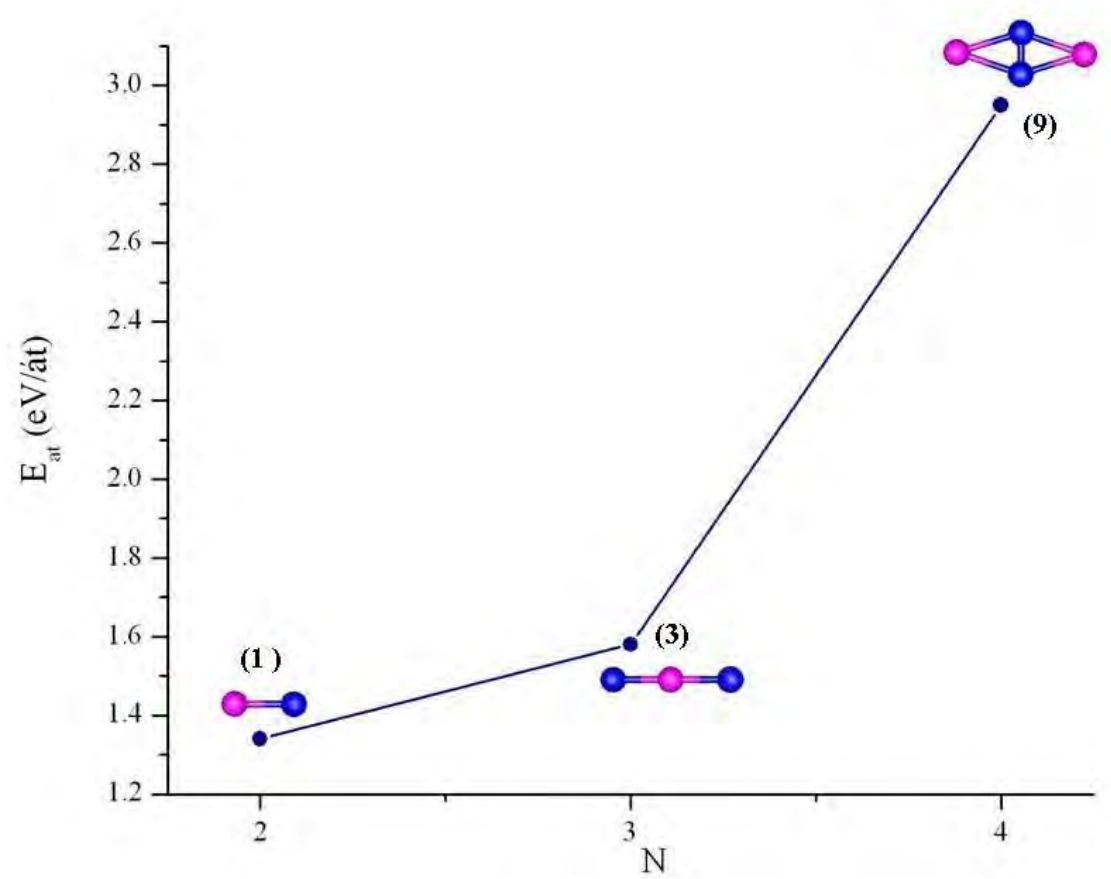

Figura 3.16.a Patrón de crecimiento del trímero (3).

En la Figura 3.16.a se muestra el patrón de crecimiento del tetrámero romboidal (9), donde se evidencia un reacomodamiento muy grande de átomos con respecto al trímero (3) (para mayores detalles de las estructuras, véanse Figuras 3.1 y 3.2) y, observando las cargas ESP del confórmero (9) informadas en la Tabla 3.8, se observa que las mismas resultaron muy cercanas a cero. También se ve que en este crecimiento la cantidad de enlaces del tetrámero es mayor que en los crecimientos mostrados en las Figuras 3.16.b y 3.16.c.

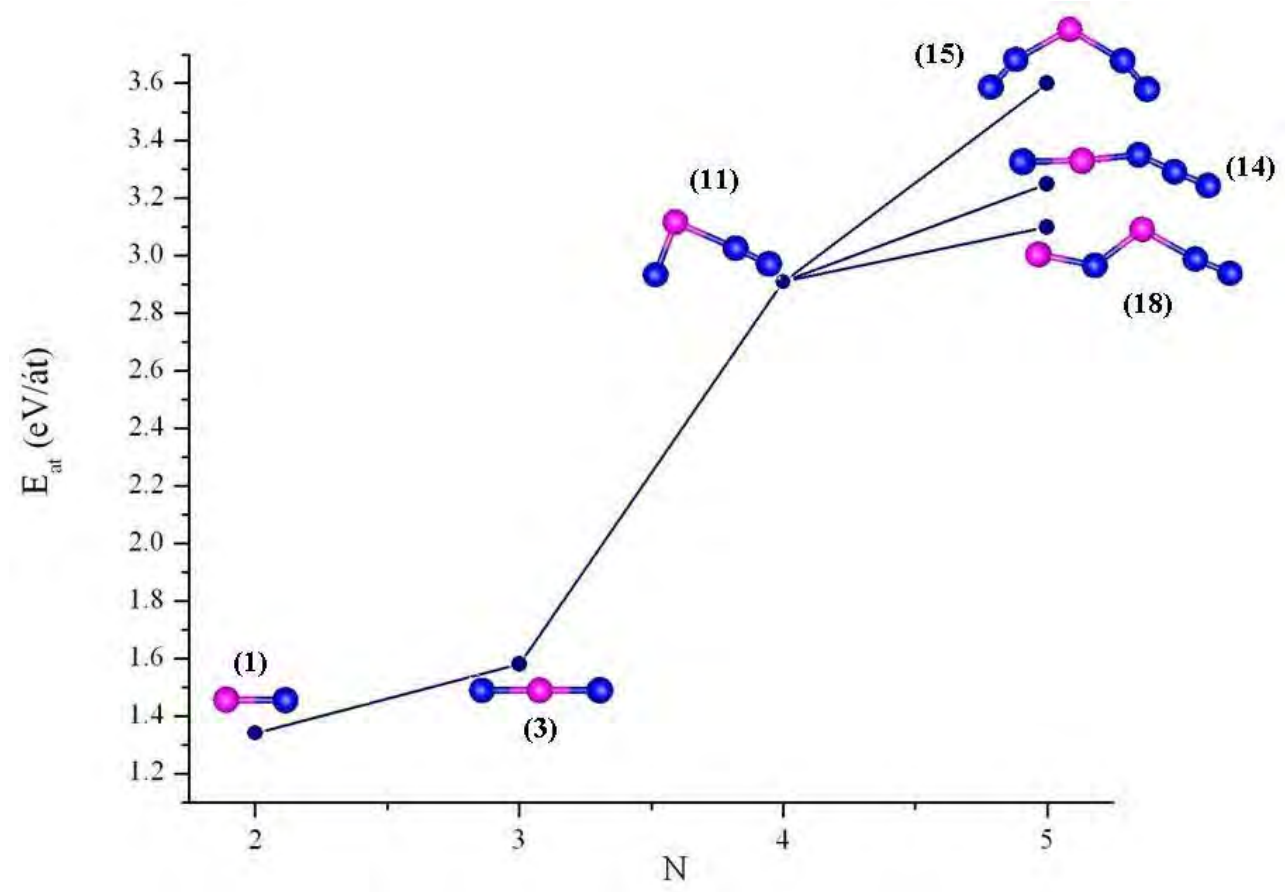

Figura 3.16.b Patrón de crecimiento del trímero (3). 
Con respecto al patrón de crecimiento mostrado en la Figura 3.16.b, se puede observar que se encontraron tres posibilidades de crecimiento de los confórmeros (1)-(3)-(11), los pentámeros (15), (14) y (18), en orden decreciente de energía de atomización. A pesar de que (15) posee la mayor $\mathrm{E}_{\mathrm{a}}$, $\mathrm{y}$ podría considerarse la mejor posibilidad de crecimiento encontrada para los agregados (1)-(3)-(11), este agregado posee cargas ESP muy bajas, Tabla 3.9, lo que justifica y su menor reactividad su gran estabilidad con respecto a los otros agregados. Debido a esto, se propone a (14) como un mejor candidato para continuar el crecimiento.

El patrón de crecimiento del agregado (3), mostrado en la Figura 3.16.b, permite concluir que cuando un nuevo átomo de nitrógeno se une a un átomo de nitrógeno ya presente en el tetrámero, lo hará con el que exhiba la mayor carga ESP negativa. Así, un nuevo átomo de nitrógeno se unirá al átomo N1 en el agregado (11), en lugar de hacerlo al átomo N3, dado que posee la mayor carga ESP, hecho que conduce a una mayor energía de atomización para el pentámero resultante.

Por otro lado, se observa que cuando un átomo de aluminio se une al átomo $\mathrm{N} 1$ en el agregado (11), el agregado resultante muestra una energía de atomización considerablemente menor que los agregados (14) y (15). Este hecho puede asociarse a la menor energía del enlace Al-N comparada con el enlace N-N.

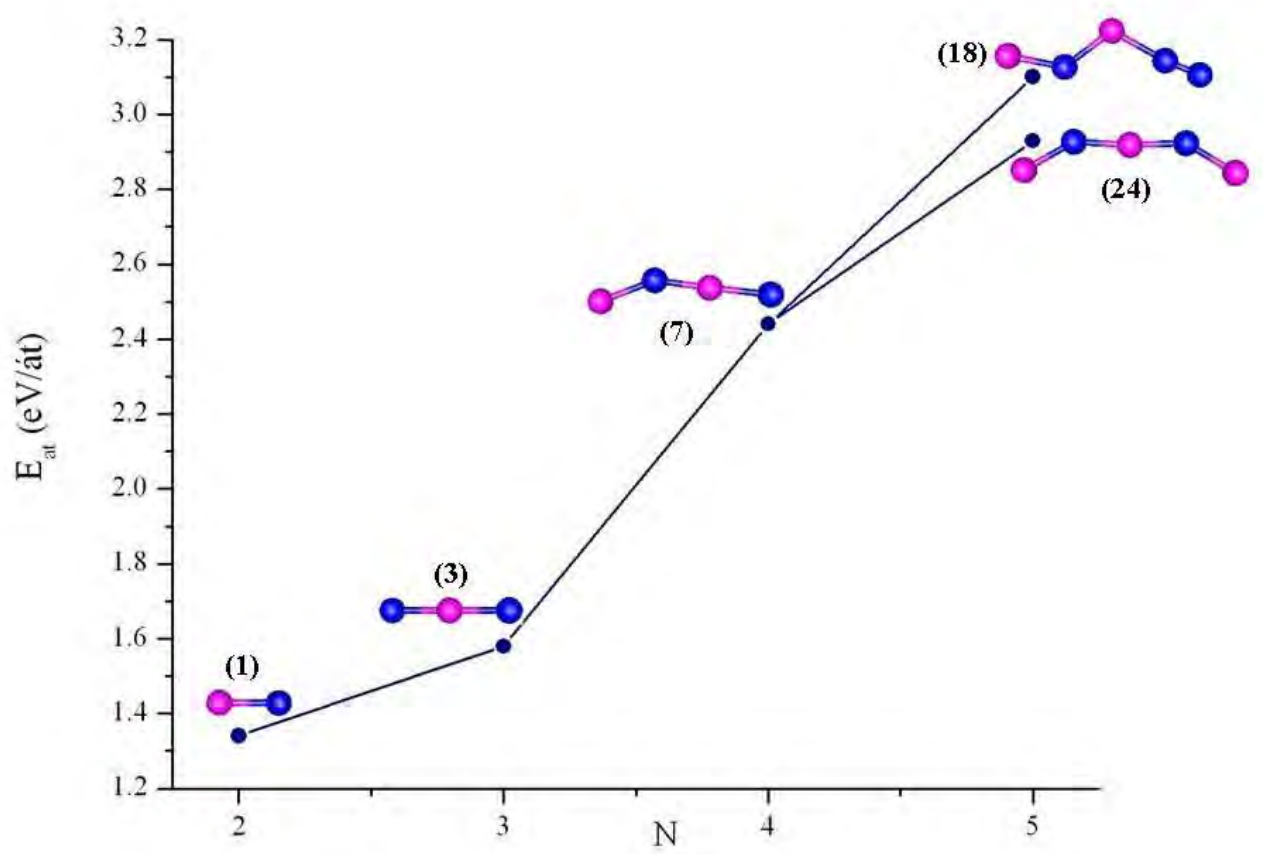

Figura 3.16.c Patrón de crecimiento del trímero (3).

En el patrón de crecimiento mostrado en la Figura 3.16.c se observa que el patrón de crecimiento más probable para los agregados (1)-(3)-(7) es el que finaliza en el confórmero (18), mostrando una preferencia del sistema a unir átomos de $\mathrm{N}$ en vez de átomos de $\mathrm{Al}$ y a unirlos en la zona del confórmero donde haya carga ESP negativa (Tabla 3.7). Analizando los resultados del confórmero (18) en la Tabla 3.7 y en las Figuras 3.9 y 3.13, se observa que el átomo de nitrógeno atacante prefiere zonas de MEP negativo (que se corresponden con las zonas de cargas ESP negativas), donde haya una mayor diferencia en la densidad de espín, es decir, una mayor cantidad de electrones desapareados.

Asimismo, ambos agregados pueden continuar su crecimiento, posiblemente por N1 en (18) o por los átomos de nitrógeno simétricos de (24). 


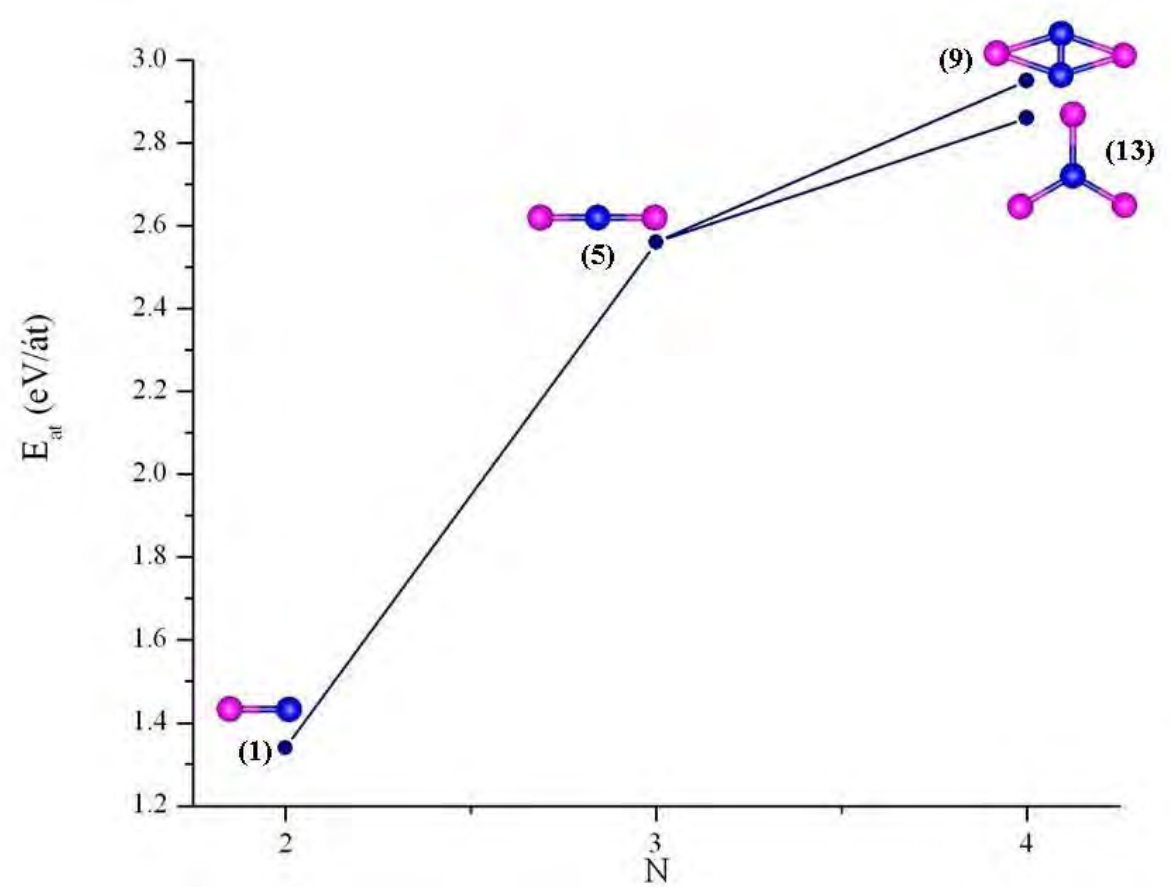

Figura 3.17. Patrón de crecimiento del trímero (5).

Con respecto al patrón de crecimiento mostrado en la Figura 3.17, se evidencia que el trímero (5) crece hasta el tetrámero (9), donde la cantidad de uniones es mayor con respecto a (13) y donde las zonas susceptibles de ataque electrofílico están sobre átomos de nitrógeno (Tabla 3.7 y Figura 3.12).

Finalmente, los patrones de crecimiento más probables propuestos para cada trímero, son:

1) $\rightarrow(2) \rightarrow(10)$

(Figura 3.15)

$(1) \rightarrow(3) \rightarrow(11) \rightarrow(14)$

(Figura 3.16.b)

(1) $\rightarrow$ (5) $\rightarrow$ (9)

(Figura 3.17)

Por lo expresado anteriormente, podemos concluir que el patrón de crecimiento más probable es el que se muestra en la Figura 3.16.b, dado que es el que sigue el mayor aumento en la energía de atomización. Entonces, podemos decir que los clusters de $\mathrm{Al} / \mathrm{N}$ continuarán creciendo a partir del pentámero (14). También, todos los patrones de crecimiento tienden a pentámeros planos no lineales, con excepción del crecimiento del trímero (2) (Figura 3.15) y el trímero (5) (Figura 3.16), que sólo crecen hasta el tetrámero.

De las Figuras 3.11 a 3.14 y teniendo en cuenta los caminos de crecimiento, podemos ver que tanto el aluminio como el nitrógeno que se unen al agregado prefieren una zona de MEP negativo, comportándose como electrófilos. Observando las Figuras 3.7 a 3.10, donde se muestran las densidades de espín, así como también las Tablas 3.7 a 3.9, podemos establecer que el electrófilo atacante no siempre se une a una zona donde haya electrones desapareados, sino que también ataca zonas donde se encuentran pares libres. Asimismo, se favorece la formación de enlaces N-N sobre Al-N y Al-Al; y sólo cuando no es posible formar enlaces $\mathrm{N}$ $\mathrm{N}$ se forman uniones Al-N para continuar el crecimiento.

\subsection{Conclusiones}

Se han obtenido las geometrías, energías de atomización, frecuencias de vibración armónicas, potenciales electrostáticos moleculares, cargas ESP, densidades de espín y patrones de 
crecimiento de clusters de $\mathrm{Al}$ y N. En los trímeros y tetrámeros las energías de atomización encontradas son mayores que las reportadas en literatura, lo que indica que la metodología seguida para lograr estructuras estables es exitosa.

El dímero AlN presenta un estado electrónico triplete con una $\mathrm{E}_{\mathrm{at}}$ de $1.36 \mathrm{eV} /$ át. El trímero más estable es AlNN (2) con un electrón desapareado y geometría lineal. El tetrámero de mayor $\mathrm{E}_{\text {at }}$ es AlNNN (10) de capa cerrada y geometría lineal. En los pentámeros el agregado más estable es NNAINN (15), caracterizado por un estado electrónico cuatriplete y una $\mathrm{E}_{\mathrm{at}} \mathrm{de}$ $3.60 \mathrm{eV} / a ́ t$, de geometría plana no lineal, agregado extremadamente estable, lo que se evidencia por sus cargas ESP bajas, además de su energía de atomización alta. En todos los casos el agregado más estable es aquel que posee $N-1$ átomos de nitrógeno en su estructura, siendo $N$ el número total de átomos.

Analizando los modos vibracionales de todos los agregados, encontramos que los movimientos de wagging, scissoring y twisting se encuentran entre $36-76 \mathrm{~cm}^{-1}$; los

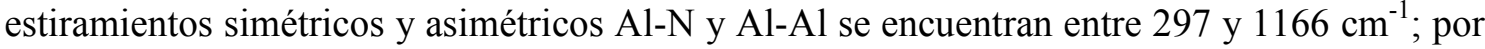
último, los estiramientos simétricos y asimétricos N-N se encuentran a frecuencias altas, de $1169 \mathrm{~cm}^{-1}$ a $2309 \mathrm{~cm}^{-1}$.

Se encontró que la mayoría de los agregados constituidos por aluminio y nitrógeno crece hacia un pentámero plano no lineal. Los clusters que crecen a partir de los trímeros AINN (2) y AlNAl (5), sólo crecen hasta el tetrámero. Por otro lado, el confórmero AlAlN (4) no presenta patrón de crecimiento. El patrón de crecimiento más probable es el que corresponde a los confórmeros AlN (1) $\rightarrow$ NAlN (3) $\rightarrow$ NAINN (11) $\rightarrow$ NAlNNN (14), donde el átomo que ataca prefiere una zona de MEP negativo y de mayor carga ESP, favoreciendo la formación de uniones N-N. Además, tanto el aluminio como el nitrógeno se comportan como electrófilos que no atacan siempre en zonas donde se encuentran electrones desapareados. Podemos concluir, entonces, que las cargas ESP, los MEP y las densidades de espín son herramientas útiles para encontrar y entender el crecimiento de este tipo de agregados. 


\section{Estudio de la estructura, modos vibracionales y patrones de crecimiento de clusters de boro y nitrógeno}

Clusters constituidos por boro y nitrógeno han sido estudiados desde hace algunos años, tanto de manera teórica como experimental ${ }^{76}$. Los agregados binarios de $\mathrm{B} / \mathrm{N}$, han atraído mucha atención como precursores en el crecimiento de la película delgada de $\beta$-BN usando deposición por vapor o técnicas utilizando plasma. Hay un marcado interés en sintetizar este tipo, esfalerita (o fase cristalina $\beta$ ), que es isoestructural e isoelectrónico con el diamante cúbico y posee propiedades fisicoquímicas excepcionales. También se realizan estudios en agregados de $\mathrm{B} / \mathrm{N}$ con el objeto de sintetizar y caracterizar análogos químicos de fulerenos en los cuales los átomos de carbono se sustituyen por boro y nitrógeno.

Con respecto a los trabajos teóricos encontrados en literatura, la mayoría se centra en el conocimiento de la estructura de los agregados binarios, neutros e iónicos, y propiedades vibracionales de los mismos. Martin y colaboradores ${ }^{77}$ han estudiado en diversos trabajos agregados de $\mathrm{B}_{2} \mathrm{~N}, \mathrm{BN}_{2}, \mathrm{BN}$ y $\mathrm{B}_{\mathrm{n}} \mathrm{N}_{\mathrm{n}}(\mathrm{n}=3-10)$, lineales y cíclicos, usando Hartree-Fock con la base 6-31G* y CCSD con el conjunto de bases cc-pVTZ, enfocándose en sus estructuras y modos vibracionales. $\mathrm{Xu}$ y coautores ${ }^{78}$ estudiaron diversas conformaciones de clusters neutros de capa cerrada $\mathrm{B}_{2} \mathrm{~N}_{2}$ de estado electrónico singlete, con el objeto de conocer su superficie de energía potencial, usando MP2 y bases $6-311 \mathrm{G}(\mathrm{d})$. Millev y colaboradores ${ }^{79}$ estudiaron clusters de $\mathrm{B}_{\mathrm{x}} \mathrm{N}_{\mathrm{x}}(\mathrm{x}=1-4,12,15,30)$, con el objeto de conocer sus estructuras como posibles análogos de fulerenos, mediante HF y el funcional BVWN de la teoría del funcional, con bases $6-31 \mathrm{G}(\mathrm{d})$. Giuffreda y colaboradores ${ }^{76}$ estudiaron las propiedades de clusters binarios iónicos usando el funcional híbrido de intercambio y correlación B3LYP y el conjunto de funciones base cc-pVDZ. Burril y Grein ${ }^{71}$ estudiaron las propiedades estructurales de clusters del tipo $\mathrm{X}_{2} \mathrm{Y}_{2}(\mathrm{X}=\mathrm{B}, \mathrm{Al}, \mathrm{Ga} ; \mathrm{Y}=\mathrm{N}, \mathrm{P}, \mathrm{As})$ usando DFT, el funcional B3LYP y el conjunto de funciones base $6-311+\mathrm{G}(3 \mathrm{df})$.

En el presente capítulo informamos los resultados obtenidos para agregados binarios de $\mathrm{B}_{n} \mathrm{~N}_{m}$, con $n=1-4, m=1-4$ y $n+m \leq 5$. Se reportan propiedades estructurales, energías de atomización y frecuencias vibracionales para los agregados más estables.

El estudio de los patrones de crecimiento se realizó conformando los agregados más allá del dímero por la adición de átomos de $\mathrm{B}$ o $\mathrm{N}$. El análisis de los patrones de crecimiento se llevó a cabo por medio de potenciales electrostáticos moleculares (MEP), densidades de espín y cargas derivadas de potenciales electrostáticos (cargas ESP).

\subsection{Estructuras, energías y modos vibracionales}

\section{Dímero $B N$}

Se encontró que el estado fundamental del dímero BN posee estado electrónico triplete (Tabla 4.1) con una energía de atomización de $2.41 \mathrm{eV} /$ át y una distancia de enlace B-N de $1.32 \AA$. Las geometrías de equilibrio se muestran en la Figura 4.1.

\section{Trímeros}

Se calcularon cuatro trímeros de B/N. Se encontró que el estado fundamental de los trímeros presenta estado electrónico doblete, en todos los casos, y sus geometrías son lineales. Se listan las $E_{a t}$, multiplicidades de espín $\left(\mathrm{M}_{\mathrm{S}}\right)$ y frecuencias vibracionales $\left(\omega_{\mathrm{e}}\right)$ en las Tablas 4.1 y 4.2 , respectivamente. Se observa que (4) es el más estable de la serie, constituido por dos átomos de boro y uno de nitrógeno (Figura 4.1). La energía de atomización aumenta en el siguiente 
orden: (5) < (3) < (2) < (4) (Las energías de (3) y (5) expresadas con tres decimales son, respectivamente: 3.084 y 3.077 eV/át).

Tabla 4.1. Multiplicidades de espín $\left(\mathrm{M}_{\mathrm{S}}\right)$, energías de atomización $\left(\mathrm{E}_{\mathrm{at}}\right)$ en eV/át, del dímero y trímeros.

\begin{tabular}{c|c|c} 
Agregado & $\mathbf{M}_{\mathbf{S}}$ & $\mathbf{E}_{\mathbf{a t}}$ \\
\hline $\mathbf{( 1 )}$ & 3 & 2.41 \\
\hline & $3^{77}$ & $2.28^{77}$ \\
\hline & $3^{80}$ & \\
\hline $\mathbf{( 2 )}$ & 2 & 3.45 \\
\hline & $2^{77}$ & $2.95^{77}$ \\
\hline $\mathbf{( 3 )}$ & 2 & 3.08 \\
\hline $\mathbf{( 4 )}$ & 2 & 3.86 \\
\hline & $2^{77}$ & $3.47^{77}$ \\
\hline $\mathbf{( 5 )}$ & 2 & 3.08
\end{tabular}

Se puede observar a partir de la Tabla 4.1 que, al comparar los resultados encontrados siguiendo patrones de crecimiento, con los de Martin y coautores ${ }^{77}$, se obtuvieron energías de atomización mayores en este trabajo. Estos autores usaron el método MP4/6-31G* para el cálculo de las energías, y las geometrías fueron optimizadas a nivel HF/6-31G*. Por otro lado, los resultados están en acuerdo con lo reportado por Bredohl y coautores ${ }^{80}$.

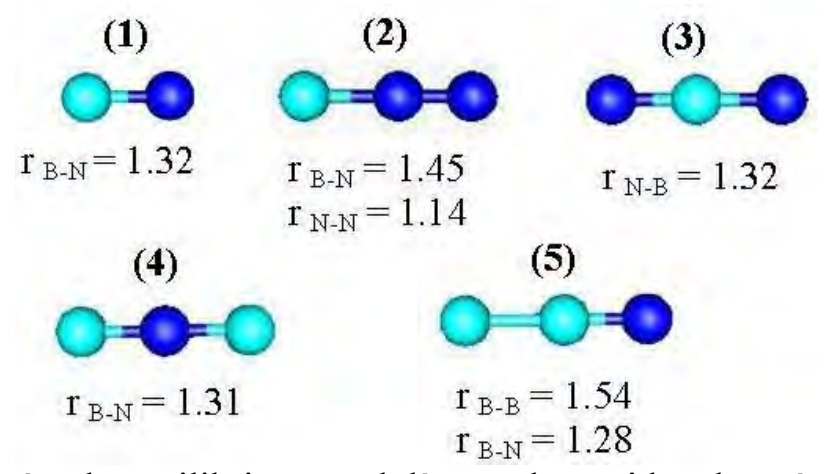

Figura 4.1. Geometrías de equilibrio para el dímero de partida y los trímeros. Las distancias (r) están expresadas en $\AA$, los ángulos planos son $180^{\circ}$, para todos los casos.

Con respecto a los parámetros geométricos de los trímeros, se puede observar que la distancia B-N está entre 1.28 y $1.32 \AA$ para la mayoría de los casos, con excepción del agregado (2), en el que esta distancia es mayor, por encontrarse adyacente a una unión N-N de carácter triple. La distancia B-N encontrada por los autores de la referencia 77 es de similar magnitud a la informada en la Figura 4.1. No obstante, la distancia N-N informada por los mismos autores es mucho mayor (1.27 $\AA$ ), lo que sugiere un enlace doble N-N. Por otro lado, Mileev y colaboradores $^{79}$, utilizando BVWN/6-31G(d), encontraron una distancia B-N para el dímero de $1.24 \AA$, considerablemente menor a lo encontrado en este trabajo. Bredohl y coautores ${ }^{80}$ citan una distancia experimental de $1.281 \AA$ A para el dímero BN. 
Tabla 4.2. Frecuencias vibracionales $\left(\omega_{\mathrm{e}}\right)$ del dímero y los trímeros en $\mathrm{cm}^{-1}$. El * significa que el modo es doblemente degenerado.

\begin{tabular}{c|c} 
Agregado & $\boldsymbol{\omega}_{\mathbf{e}}$ \\
\hline $\mathbf{( 1 )}$ & 1564 \\
\hline & $1515^{80}$ \\
\hline $\mathbf{( 2 )}$ & $90,215,838,1957$ \\
\hline & $1221,1290,1760^{77}$ \\
\hline $\mathbf{( 3 )}$ & $148,306,1050,1799$ \\
\hline $\mathbf{4})$ & $122^{*}, 1196,1329$ \\
\hline & $82,1245,2271^{77}$ \\
\hline $\mathbf{( 5 )}$ & $166^{*}, 9131791$
\end{tabular}

Respecto a los modos vibracionales del dímero y los trímeros formados por B/N (Tabla 4.2), en frecuencias bajas encontramos movimientos de tipo wagging, en frecuencias intermedias estiramientos simétricos B-N y los estiramientos asimétricos B-B y B-N se encuentran a más de $1300 \mathrm{~cm}^{-1}$. Por otro lado, los estiramientos simétricos y asimétricos N-N se encuentran a más de $1900 \mathrm{~cm}^{-1}$. Se ve una diferencia importante entre los modos vibracionales informados en la referencia 77 y los encontrados en este trabajo y un muy buen acuerdo con la frecuencia obtenida para el dímero de manera experimental y citada por Bredohl y colaboradores ${ }^{80}$.

\section{Tetrámeros}

Se obtuvieron 12 tetrámeros estables, 8 de los cuales eran inicialmente lineales, 2 romboidales y 2 cuadrados. En la Figura 4.2 se muestran las geometrías de equilibrio obtenidas. En la Tabla 4.3 se listan las $\mathrm{E}_{\mathrm{at}}$ correspondientes y multiplicidades de espín. Los modos vibracionales se listan en la Tabla 4.4. Para completar el estudio, se calcularon los agregados BNBN rombo y cuadrado ((7) y (8), respectivamente) y BBNN rombo y cuadrado ((16) y (17), respectivamente). 
(6)

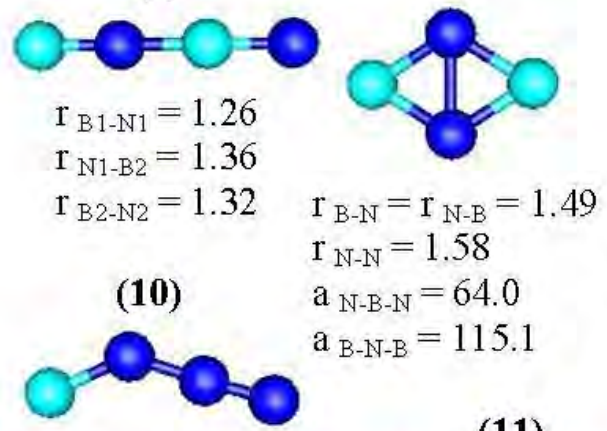

$\mathrm{r}_{\mathrm{B} 1-\mathrm{N} 1}=1.39$

$\mathrm{r}_{\mathrm{N} 1-\mathrm{N} 2}=1.22$

$\mathrm{r}_{\mathrm{N} 2-\mathrm{N} 3}=1.12$

$\mathrm{a}_{\mathrm{B} 1-\mathrm{N} 1-\mathrm{N} 2}=137.6$

(14)

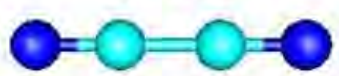

$\mathrm{r}_{\mathrm{B}-\mathrm{N}}=1.32$

$r_{B-B}=1.55$
(11)

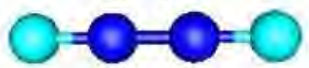

$$
\mathrm{r}_{\mathrm{B}-\mathrm{N}}=1.24
$$$$
\mathrm{r}_{\mathrm{N}-\mathrm{N}}=1.27
$$

(15)

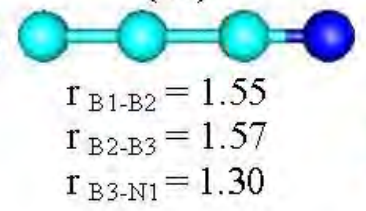

(8)

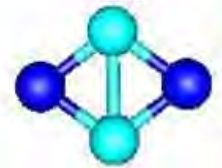

$r_{B-N}=r_{N-B}=1.41$

$\mathrm{r}_{\mathrm{B}-\mathrm{B}}=1.68$

$\mathrm{a}_{\mathrm{N}-\mathrm{B}-\mathrm{N}}=106.8$

$\mathrm{a}_{\mathrm{B}-\mathrm{N}-\mathrm{B}}=73.2$
(9)

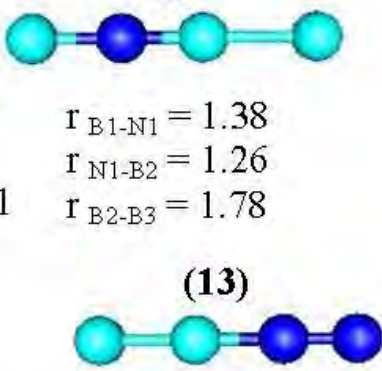

(12)

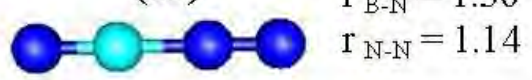

$$
\begin{aligned}
& r_{\text {B-B }}=1.49 \\
& r_{\text {B-N }}=1.36 \\
& r_{\text {N-N }}=1.14
\end{aligned}
$$

$$
\begin{aligned}
& \mathrm{r}_{\mathrm{N} 1-\mathrm{B} 1}=1.26 \\
& \mathrm{r}_{\mathrm{B} 1-\mathrm{N} 2}=1.40 \\
& \mathrm{r}_{\mathrm{N} 2-\mathrm{N} 3}=1.11
\end{aligned}
$$

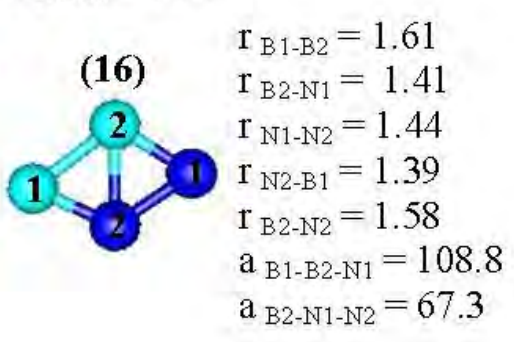

(17)

$$
\begin{aligned}
& r_{\mathrm{B} 1-\mathrm{B} 2}=1.57 \\
& \mathrm{r}_{\mathrm{B} 2-\mathrm{N} 1}=1.34 \\
& \mathrm{r}_{\mathrm{N} 1-\mathrm{N} 2}=1.75 \\
& 2 \mathrm{r}_{\mathrm{N} 2-\mathrm{B} 1}=1.33 \\
& \mathrm{r}_{\mathrm{B} 1-\mathrm{N} 1}=1.64 \\
& \mathrm{a}_{\mathrm{B} 1-\mathrm{B} 2 \mathrm{~N} 1}=67.9 \\
& \mathrm{a}_{\mathrm{B} 2-\mathrm{N} 1-\mathrm{N} 2}=101.6 \\
& \mathrm{~d}_{\mathrm{B} 1-\mathrm{N} 2-\mathrm{N} 1-\mathrm{B} 2}=32.4
\end{aligned}
$$

Figura 4.2. Geometrías de equilibrio de los tetrámeros. Las distancias (r) están expresadas en $\AA ̊$, los ángulos diedros que no se especifican son cero y los planos (a) $180^{\circ}$.

De la Figura 4.2, se desprende que los agregados que eran inicialmente lineales permanecieron así, con excepción de (10), que se deformó a no lineal, con un ángulo de $137.6^{\circ}$ en su estructura. Los agregados que inicialmente eran cuadrados se deformaron a estructuras menos simétricas (agregados (8) y (17)). Con respecto a las distancias de enlace, la unión B-B se encuentra entre 1.49 y $1.78 \AA$, la distancia B-N entre 1.24 y 1.49 , y la unión N$\mathrm{N}$ entre 1.11 y 1.75 , encontrándose uniones dobles y triples en los agregados lineales y en (10), y uniones simples en los agregados cíclicos, (16) y (17). Asimismo, Mileev y coautores ${ }^{79}$ encontraron una distancia B-N para el tetrámero lineal alternado de $1.38 \AA$ (6) y de 1.39 Å para el isómero cíclico alternado (7) y (8). 
Tabla 4.3. Multiplicidades de espín $\left(\mathrm{M}_{\mathrm{S}}\right)$ y energías de atomización $\left(\mathrm{E}_{\mathrm{at}}\right)$ en eV/át, de los tetrámeros.

\begin{tabular}{c|c|c} 
Agregado & $\mathbf{M}_{\mathbf{S}}$ & $\mathbf{E}_{\mathbf{a t}}$ \\
\hline $\mathbf{( 6 )}$ & 3 & 4.09 \\
\hline & $3^{80}$ & \\
\hline $\mathbf{( 7 )}$ & 3 & 3.34 \\
\hline $\mathbf{( 8 )}$ & 3 & 3.78 \\
\hline $\mathbf{( 9 )}$ & 1 & 3.69 \\
\hline $\mathbf{( 1 0 )}$ & 1 & 3.87 \\
\hline $\mathbf{( 1 1 )}$ & 3 & 3.57 \\
\hline & $1^{78}$ & \\
\hline $\mathbf{( 1 2 )}$ & 1 & 4.22 \\
\hline $\mathbf{( 1 3 )}$ & 3 & 3.60 \\
\hline $\mathbf{( 1 4 )}$ & 3 & 3.70 \\
\hline $\mathbf{( 1 5 )}$ & 3 & 3.26 \\
\hline $\mathbf{( 1 6 )}$ & 3 & 3.52 \\
\hline $\mathbf{( 1 7 )}$ & 1 & 3.32
\end{tabular}

El agregado más estable, de estado electrónico singlete y con tres átomos de nitrógeno y uno de boro en su estructura, es el agregado (12). El aumento en la estabilidad de los agregados, indicado por el aumento de la $E_{a t}$, se presenta de la siguiente manera: $(\mathbf{1 5})<$ (17) $<$ (7) $<$ (16) $<$ (11) $<$ (13) $<$ (9) $<$ (14) $<$ (8) $<$ (10) $<$ (6) $<$ (12). También se observa una preferencia de los sistemas a tener dos electrones desapareados.

Cabe destacar que Burril y Grein ${ }^{71}$ obtienen el tetrámero $\mathrm{B} / \mathrm{N}$ alternado de estado electrónico triplete (el agregado (6) en este trabajo) como más estable. Por otro lado, Xu y colaboradores $^{78}$ reportan como más estable el tetrámero lineal similar a (11) de la Figura 4.2, de estado electrónico singlete, con una distancia N-B de $1.26 \AA$ y una distancia B-B de 1.76 $\AA$. 
Tabla 4.4. Frecuencias vibracionales $\left(\omega_{\mathrm{e}}\right)$ de los tetrámeros en $\mathrm{cm}^{-1} . *$ significa que el modo es doblemente degenerado.

\begin{tabular}{c|c} 
Agregado & $\boldsymbol{\omega}_{\mathbf{e}}$ \\
\hline $\mathbf{( 6 )}$ & $174,178,433,468,850,1730,2023$ \\
\hline $\mathbf{( 7 )}$ & $113,360,540,681,799,1132$ \\
\hline $\mathbf{( 8 )}$ & $719,750,787,924,1281,1317$ \\
\hline $\mathbf{( 9 )}$ & $81^{*}, 193^{*}, 581,1134,1979$ \\
\hline $\mathbf{( 1 0 )}$ & $172,573,580,977,1535,2330$ \\
\hline $\mathbf{( 1 )}$ & $217^{*}, 380^{*}, 994,1923,2135$ \\
\hline $\mathbf{( 1 2 )}$ & $181^{*}, 522^{*}, 788,1961,2274$ \\
\hline $\mathbf{( 1 3 )}$ & $152,181,484,505,795,1552,2038$ \\
\hline $\mathbf{( 1 4 )}$ & $157^{*}, 389^{*}, 675,1420,1827$ \\
\hline $\mathbf{( 1 5 )}$ & $143,163,357,416,642,1250,1801$ \\
\hline $\mathbf{( 1 6 )}$ & $392,559,655,897,1251,1308$ \\
\hline $\mathbf{( 1 7 )}$ & $207,316,441,981,1281,1518$ \\
\hline
\end{tabular}

En el caso de los modos vibracionales de los tetrámeros (Tabla 4.4), en frecuencias bajas se presentan movimientos de tipo twisting, wagging y scissoring; en frecuencias intermedias estiramientos B-N y B-B asimétricos y simétricos y en frecuencias mayores a $2000 \mathrm{~cm}^{-1} \mathrm{se}$ encuentran estiramientos N-N simétricos y asimétricos.

\section{Pentámeros}

Se obtuvieron 28 pentámeros de los cuales 10 son lineales y 18 no lineales. Como punto de partida para formar los pentámeros se tomaron los tetrámeros antes descriptos, tanto lineales como cíclicos, haciéndolos crecer con átomos de boro y nitrógeno en todos los sitios posibles. En algunos casos no se obtuvieron estructuras estables. En las Figuras 4.3 y 4.4, y Tablas 4.5 y 4.6 se muestran los resultados obtenidos. 
(18)

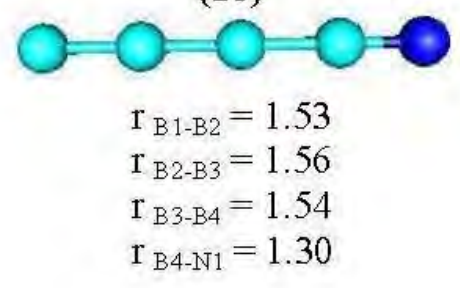

(19)

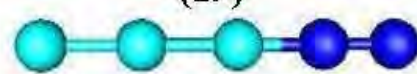

$\mathrm{r}_{\mathrm{B} 1-\mathrm{B} 2}=1.53$

$\mathrm{r}_{\mathrm{B} 2-\mathrm{B} 3}=1.50$

$\mathrm{r}_{\mathrm{B} 3-\mathrm{N} 1}=1.35$

$\mathrm{r}_{\mathrm{N} 1-\mathrm{N} 2}=1.14$

(22)

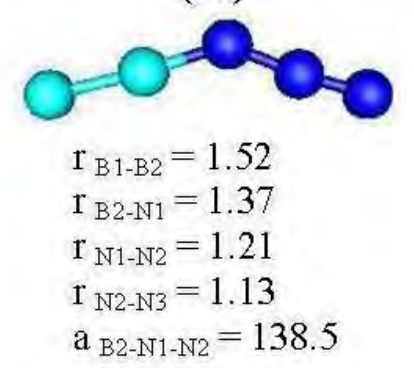

(24)

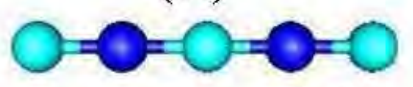

$\mathrm{r}_{\mathrm{B} 1-\mathrm{N} 1}=\mathrm{r}_{\mathrm{N} 2-\mathrm{B} 3}=1.30$

$\mathrm{r}_{\mathrm{N} 1-\mathrm{B} 2}=\mathrm{r}_{\mathrm{B} 2-\mathrm{N} 2}=1.31$

(27)

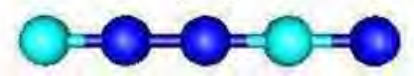

$\mathrm{r}_{\mathrm{B} 1-\mathrm{N} 1}=1.26$

$\mathrm{r}_{\mathrm{N} 1-\mathrm{N} 2}=1.24$

$\mathrm{r}_{\mathrm{N} 2-\mathrm{B} 2}=1.29$

$\mathrm{r}_{\mathrm{B} 2-\mathrm{N} 3}=1.35$

(30)

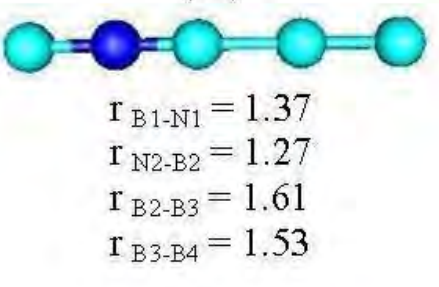

(25)

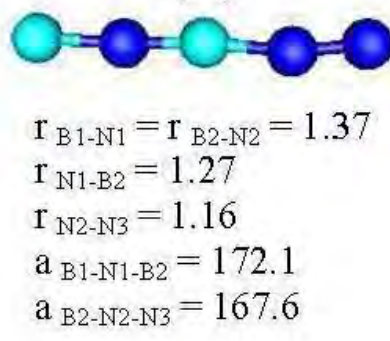

(28)

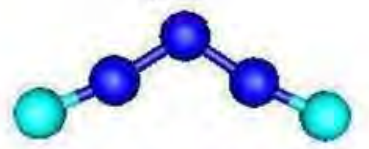

$\mathrm{r}_{\mathrm{B}-\mathrm{N}}=1.25$

$\mathrm{r}_{\mathrm{N}-\mathrm{N}}=1.30$

$\mathrm{a}_{\mathrm{N}-\mathrm{N}-\mathrm{N}}=113.9$

(20)

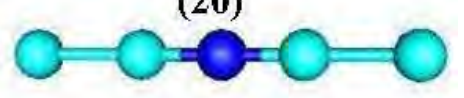

$\mathrm{r}_{\mathrm{B}-\mathrm{B}}=1.73$
$\mathrm{r}_{\mathrm{B}-\mathrm{N}}=1.30$

(23)

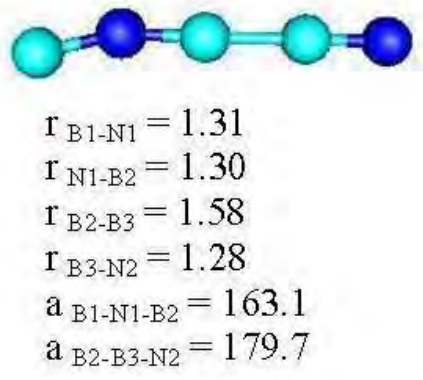

(26)

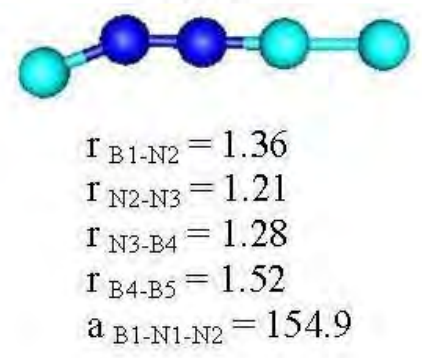

(29)

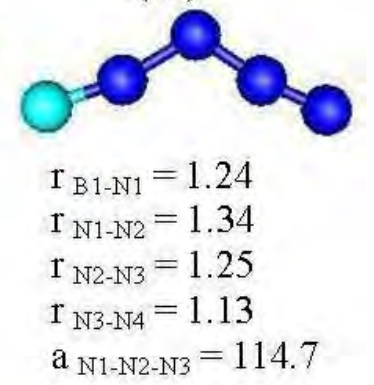

(31)

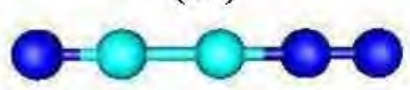

$$
\begin{aligned}
& \mathrm{r}_{\mathrm{N} 1-\mathrm{B} 1}=1.28 \\
& \mathrm{r}_{\mathrm{B} 1-\mathrm{B} 2}=1.52 \\
& \mathrm{r}_{\mathrm{B} 2-\mathrm{N} 2}=1.36 \\
& \mathrm{r}_{\mathrm{N} 2-\mathrm{N} 3}=1.14
\end{aligned}
$$$$
\mathrm{r}_{\mathrm{N}-\mathrm{B}}=1.30
$$$$
r_{B-B}=1.57
$$

(32)

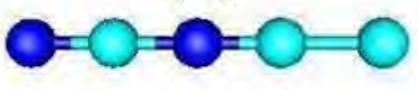

$$
\begin{aligned}
& \mathrm{r}_{\mathrm{N1}-\mathrm{B} 1}=1.33 \\
& \mathrm{r}_{\mathrm{B} 1-\mathrm{N} 2}=1.32 \\
& \mathrm{r}_{\mathrm{N2}-\mathrm{B} 2}=1.29 \\
& \mathrm{r}_{\mathrm{B} 2-\mathrm{B} 3}=1.55
\end{aligned}
$$

(33)

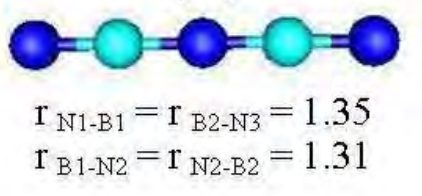

Figura 4.3. Geometrías de equilibrio de los pentámeros (18) a (33). Las distancias (r) están expresadas en $\AA$, los ángulos planos (a) y los diedros (d), en ${ }^{\circ}$. Los ángulos planos que no se especifican valen $180^{\circ}$. 
(34) (35)

(35) (36)
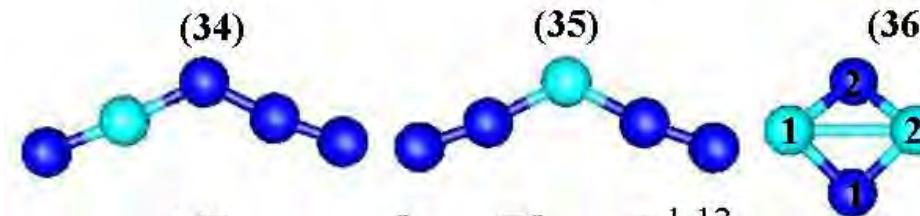

(3)

$\mathrm{r}_{\mathrm{B} 1-\mathrm{N} 1}=\mathrm{r}_{\mathrm{B} 1-\mathrm{N} 2}=1.43$

$\mathrm{r}_{\mathrm{N} 1-\mathrm{B} 2}=\mathrm{r}_{\mathrm{B} 2-\mathrm{N} 2}=1.45$

$\mathrm{r}_{\mathrm{N} 1-\mathrm{B} 1}=1.31$

$\mathrm{r}_{\mathrm{N} 1-\mathrm{N} 2}=\mathrm{r}_{\mathrm{N} 3-\mathrm{N} 4}=1.13$

$r_{B 2-B 3}=1.96$

$r_{B 2-B 3}=1.75$

$\mathrm{a}_{\mathrm{B} 1-\mathrm{N} 1-\mathrm{B} 2}=85.5$

$\mathrm{a}_{\mathrm{N} 1-\mathrm{B} 2 \mathrm{-N} 2}=78.1$

$\mathrm{r}_{\mathrm{B} 1-\mathrm{N} 2}=1.38$

$\mathrm{r}_{\mathrm{N} 2-\mathrm{B} 1}=\mathrm{r}_{\mathrm{B} 1-\mathrm{NT} 3}=1.38$

$\mathrm{a}_{\mathrm{N} 2-\mathrm{B} 2-\mathrm{B} 3}=140.5$

$\mathrm{r}_{\mathrm{N} 2-\mathrm{N} 3}=1.23$

$\mathrm{a}_{\mathrm{N} 2-\mathrm{B} 1-\mathrm{N} 3}=123.9$

$\mathrm{r}_{\mathrm{N} 3-\mathrm{N} 4}=1.12$

$\mathrm{a}_{\mathrm{B} 1-\mathrm{N} 2-\mathrm{N} 3}=127.1$

(38)

$r_{B 1-B 2}=1.60$

$\mathrm{d}_{\mathrm{B} 1-\mathrm{N} 2-\mathrm{B} 2 \mathrm{-N} 1}=40.5$
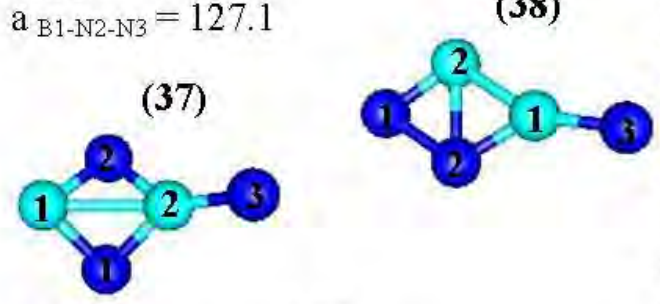

$\mathrm{r}_{\mathrm{B} 2 \mathrm{-N} 1}=1.38$

(39)

$\mathrm{r}_{\mathrm{N} 1-\mathrm{N} 2}=1.48$

$r_{\mathrm{N} 2-\mathrm{B} 1}=1,42$

$r_{B 1-\mathrm{N} 3}=1.44$

$\mathrm{r}_{\mathrm{B} 2-\mathrm{N} 1}=1.61$

$\mathrm{a}_{\mathrm{B} 1-\mathrm{B} 2-\mathrm{N} 1}=110.8$

$\mathrm{a}_{\mathrm{B} 2-\mathrm{B} 1 \mathrm{-N} 2}=64.3$

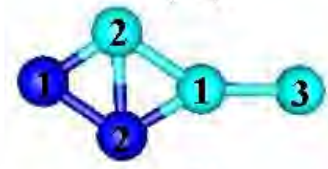

$\mathrm{r}_{\mathrm{B}-\mathrm{B}}=1.59$

$\mathrm{a}_{\mathrm{NN} 1-\mathrm{B} 1-\mathrm{N} 3}=140.8$

$\mathrm{r}_{\mathrm{N} 1-\mathrm{N} 2}=1.48$

$\mathrm{r}_{\mathrm{B} 1-\mathrm{N} 1}=\mathrm{r}_{\mathrm{B} 1-\mathrm{N} 2}=1.43$

$\mathrm{r}_{\mathrm{N} 1-\mathrm{B} 2}=\mathrm{r}_{\mathrm{B} 2-\mathrm{N} 2}=\mathrm{r}_{\mathrm{B} 2-\mathrm{N} 3}=1.45$

$\mathrm{r}_{\mathrm{B} 1-\mathrm{B} 2}=1.96$

(40)

$\mathrm{a}_{\mathrm{B} 1-\mathrm{N} 1-\mathrm{B} 2}=85.6$

$\mathrm{a}_{\mathrm{N} 1-\mathrm{B} 2-\mathrm{N} 2}=78.3$

$a_{\mathrm{N} 2-\mathrm{B} 2 \mathrm{~N} 3}=140.7$

$\mathrm{d}_{\mathrm{B} 1-\mathrm{N} 2-\mathrm{B} 2 \mathrm{~N} 1 \mathrm{~N}}=40.4$

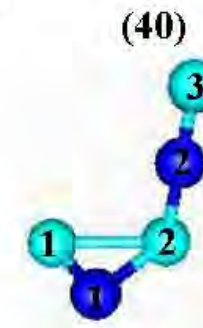

(41)

$\mathrm{r}_{\mathrm{B} 1-\mathrm{B} 2}=1.85$

$r_{B 2-\mathrm{N} 1}=1.54$

$\mathrm{r}_{\mathrm{B} 1-\mathrm{N} 2}=1.44$

$\mathrm{r}_{\mathrm{B} 2-\mathrm{N} 1}=1.37$

$\mathrm{a}_{\mathrm{B} 1-\mathrm{B} 2-\mathrm{N} 2}=112.3$

$\mathrm{a}_{\mathrm{B} 2-\mathrm{B} 1-\mathrm{N} 2}=64.1$

$a_{\mathrm{N} 2-\mathrm{B} 1-\mathrm{B} 3}=148.8$

$r_{\mathrm{B} 2-\mathrm{N} 2}=1.40$

$\mathrm{r}_{\mathrm{N} 1 \mathrm{-B} 1}=1.41$

$\mathrm{r}_{\mathrm{N} 2-\mathrm{B} 3}=1.27$

$\mathrm{a}_{\mathrm{N} 1-\mathrm{B} 2-\mathrm{N} 2}=109.1$

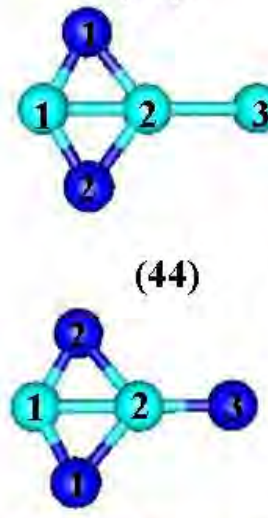

$\mathrm{r}_{\mathrm{B} 1-\mathrm{N} 1}=\mathrm{r}_{\mathrm{B} 1-\mathrm{N} 2}=1.38$

$\mathrm{d}_{\mathrm{N} 1-\mathrm{B} 2-\mathrm{N} 2-\mathrm{B} 3}=25.5$

(42)

$r_{B 1-B 2}=1.61$

$\mathrm{r}_{\mathrm{B} 2-\mathrm{B} 3}=1.78$

$\mathrm{a}_{\mathrm{B} 1-\mathrm{N} 1-\mathrm{B} 2}=67.5$

$\mathrm{a}_{\mathrm{N} 1-\mathrm{B} 2-\mathrm{N} 2}=104.7$

$\mathrm{a}_{\mathrm{N} 2-\mathrm{B} 2-\mathrm{B} 3}=127.3$

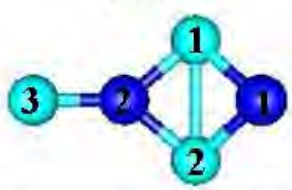

(45)

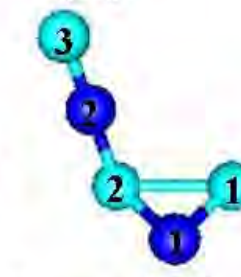

$\mathrm{r}_{\mathrm{B} 1-\mathrm{N} 2}=\mathrm{r}_{\mathrm{B} 2-\mathrm{N} 3}=1.45$

$\mathrm{r}_{\mathrm{B} 3-\mathrm{N} 4}=\mathrm{r}_{\mathrm{B} 4-\mathrm{N} 1}=1.47$

$\mathrm{r}_{\mathrm{N} 4-\mathrm{B} 5}=1.41$

$\mathrm{r}_{\mathrm{B} 1-\mathrm{B} 2}=1.89$

$\mathrm{r}_{\mathrm{B} 1-\mathrm{N} 1}=\mathrm{r}_{\mathrm{B} 1-\mathrm{N} 2}=1.36$

$\mathrm{r}_{\mathrm{N} 1-\mathrm{B} 2}=\mathrm{r}_{\mathrm{B} 2-\mathrm{N} 2}=1.54$

$\mathrm{r}_{\mathrm{B} 2-\mathrm{N} 3}=1.45$

$\mathrm{a}_{\mathrm{B} 1-\mathrm{N} 1-\mathrm{B} 2}=67.0$

$\mathrm{a}_{\mathrm{N} 1-\mathrm{B} 2-\mathrm{N} 3}=128.8$

$\mathrm{r}_{\mathrm{B} 1-\mathrm{B} 2}=1.85$

$\mathrm{a}_{\mathrm{B} 1-\mathrm{N} 2-\mathrm{B} 3}=81.04$

$a_{\text {N2-B3-N4 }}=94.36$

$\mathrm{a}_{\mathrm{B} 3-\mathrm{N} 4-\mathrm{B} 5}=139.83$

$\mathrm{a}_{\mathrm{N} 1-\mathrm{B} 2-\mathrm{N} 2}=102.6$

$\mathrm{r}_{\mathrm{B} 2 \mathrm{~N} 1}=1.54$

$\mathrm{d}_{\mathrm{B} 1-\mathrm{N} 4-\mathrm{B} 3-\mathrm{N} 2}=24.03$

(43)

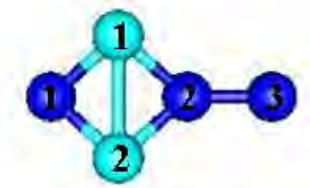

$\mathrm{r}_{\mathrm{B}-\mathrm{N}}=1.47$

$\mathrm{r}_{\mathrm{N}-\mathrm{N}}=1.32$

$\mathrm{a}_{\mathrm{B}-\mathrm{N}-\mathrm{B}} \approx 80.00$

$a_{N-B-N}=93.60$

$a_{B-N-N}=135.30$

$\mathrm{d}_{\mathrm{B}-\mathrm{N}-\mathrm{B}-\mathrm{N}}=26.80$

$\mathrm{r}_{\mathrm{B} 2-\mathrm{N} 2}=1.40$

$\mathrm{r}_{\mathrm{N} 1-\mathrm{B} 1}=1.41$

$\mathrm{r}_{\mathrm{N} 2-\mathrm{B} 3}=1.27$

$\mathrm{a}_{\mathrm{N} 1-\mathrm{B} 2-\mathrm{N} 2}=109.1$

$\mathrm{d}_{\mathrm{N} 1-\mathrm{B} 2-\mathrm{N} 2-\mathrm{B} 3}=25.5$

Figura 4.4. Geometrías de equilibrio de los pentámeros (33) a (45). Las distancias (r) están expresadas en $\AA$, los ángulos planos (a) y los diedros (d), en ${ }^{\circ}$. Los ángulos planos y diedros que no se especifican valen $180^{\circ}$ y $0.0^{\circ}$, respectivamente. 
En las Figuras 4.3 y 4.4 vemos que la distancia de enlace N-N de los pentámeros está entre 1.12 y $1.48 \AA$, la unión B-N entre 1.24 y $1.54 \AA$, la distancia B-B entre 1.50 y $1.85 \AA$, siendo los agregados cíclicos los que poseen las mayores distancias de enlace, como se encontró en los tetrámeros. En aquellos agregados con mayoría de átomos de $\mathrm{N}$, como (29), (34) y (35) se observa que la distancia N-N adyacente a un átomo de B se extiende a unión simple o doble.

Tabla 4.5. Multiplicidades de espín $\left(\mathrm{M}_{\mathrm{S}}\right)$ y energías de atomización $\left(\mathrm{E}_{\mathrm{at}}\right)$, en eV/át, de los pentámeros.

\begin{tabular}{|c|c|c|}
\hline Agregado & $\mathbf{M}_{\mathbf{S}}$ & $\mathbf{E}_{\text {at }}$ \\
\hline (18) & 4 & 3.52 \\
\hline (19) & 2 & 3.81 \\
\hline$(20)$ & 2 & 3.51 \\
\hline$(21)$ & 4 & 3.69 \\
\hline$(22)$ & 4 & 3.89 \\
\hline (23) & 2 & 4.29 \\
\hline (24) & 2 & 4.65 \\
\hline (25) & 2 & 4.35 \\
\hline (26) & 2 & 3.56 \\
\hline$(27)$ & 4 & 3.85 \\
\hline$(28)$ & 4 & 3.50 \\
\hline (29) & 2 & 3.67 \\
\hline (30) & 2 & 3.86 \\
\hline (31) & 2 & 4.10 \\
\hline (32) & 2 & 3.98 \\
\hline (33) & 4 & 4.20 \\
\hline (34) & 2 & 4.08 \\
\hline (35) & 2 & 4.28 \\
\hline (36) & 2 & 3.56 \\
\hline (37) & 4 & 3.74 \\
\hline (38) & 4 & 3.43 \\
\hline (39) & 4 & 3.60 \\
\hline$(40)$ & 6 & 3.28 \\
\hline (41) & 2 & 3.83 \\
\hline (42) & 6 & 3.34 \\
\hline (43) & 6 & 3.33 \\
\hline (44) & 4 & 3.98 \\
\hline (45) & 6 & 3.28 \\
\hline
\end{tabular}

Observamos que el agregado más estable es el lineal (24) de estado electrónico doblete, con una $\mathrm{E}_{\mathrm{at}}$ de $4.65 \mathrm{eV} / \mathrm{a}$ t, continuando con la tendencia de que los agregados lineales son más estables que los cíclicos y no lineales. Es importante mencionar que el agregado (38), se puede obtener uniendo un átomo de nitrógeno a un átomo de boro de (16) o de (17). Sólo se informa un agregado, dado que por ambos caminos se obtiene lo mismo. Otro caso interesante 
es el de los agregados (40) y (45), los menos estables de la serie, obtenidos a partir de (7) y (8), respectivamente, que resultaron ser imágenes especulares entre sí.

Tabla 4.6. Frecuencias vibracionales de los pentámeros en $\mathrm{cm}^{-1}$. $*$ significa que el modo es doblemente degenerado.

\begin{tabular}{|c|c|}
\hline Agregado & $\omega_{\mathrm{e}}$ \\
\hline (18) & $91^{*}, 221^{*}, 443^{*}, 527,1043,1419,1803$ \\
\hline (19) & $106^{*}, 282^{*}, 478^{*}, 590,1208,1550,1905$ \\
\hline (20) & $26,53,202,266,491,516,703,1418,1689$ \\
\hline (21) & $107,335,368,530,575,750,1325,1664,2306$ \\
\hline$(22)$ & $28,159,161,429 *, 565,1156,1401,1950$ \\
\hline (23) & $108,164,215,461,471,548,1341,1385,1884$ \\
\hline (24) & $81^{*}, 170^{*}, 488^{*}, 748,1214,1648,1852$ \\
\hline (25) & $39,85,108,258,301,687,1279,1888,2067$ \\
\hline (26) & $126,170,248,464,470,727,1040,1600,1918$ \\
\hline$(27)$ & $131^{*}, 345^{*}, 373^{*}, 739,1331,1729,1946$ \\
\hline (28) & $144,271,322,336,641,964,1107,1830,1928$ \\
\hline (29) & $150,274,340,508,695,929,1238,2008,2212$ \\
\hline (30) & $94,99,224,234,430,495,511,1014,1757,1850$ \\
\hline (31) & $97,111,270,320,389,522,585,1362,1870,1989$ \\
\hline (32) & $119^{*}, 333^{*}, 480^{*}, 675,1118,1664,1777$ \\
\hline (33) & $94,117,378,379,431,477,680,1123,1502,1672$ \\
\hline (34) & $137,362,445,544,609,822,1362,1845,2309$ \\
\hline (35) & $145,402,437,488,495,776,1320,1963,2105$ \\
\hline (36) & $189,199,516,569,625,713,1001,1271,1359$ \\
\hline (37) & $269,326,570,589,693,826,1008,1286,1411$ \\
\hline (38) & $205,278,363,556,605,778,952,1325,1371$ \\
\hline (39) & $185,189,363,548,609,776,914,1344,1392$ \\
\hline (40) & $142,195,353,463,495,845,1045,1169,1751$ \\
\hline (41) & $162,199,400,523,680,785,1083,1286,1326$ \\
\hline$(42)$ & $189,300,543,665,811,925,1096,1136,1393$ \\
\hline (43) & $270,359,515,632,805,902,1071,1110,1280$ \\
\hline (44) & $281,303,466,586,656,797,1164,1322,1390$ \\
\hline (45) & $143,196,354,462,494,844,1046,1166,1751$ \\
\hline
\end{tabular}

Los pentámeros $\mathrm{B} / \mathrm{N}$ presentan modos vibracionales de tipo wagging, scissoring, twisting $\mathrm{y}$ rocking a frecuencias bajas, estiramientos asimétricos y simétricos B-B y B-N en frecuencias intermedias, y en frecuencias mayores a $1900 \mathrm{~cm}^{-1}$ se ven estiramientos simétricos y asimétricos N-N. Por otro lado, se observa en la Tabla 4.6 que los agregados (40) y (45) poseen modos vibracionales muy similares, como es de esperarse, dado que son imágenes especulares uno de otro. 
Análisis de cargas derivadas del potencial electrostático y de la densidad de espín

Los análisis de cargas derivadas de potenciales electrostáticos (cargas ESP) y de la densidad de espín, se realizaron al mismo nivel de teoría antes descripto. En las Tablas 4.7 a 4.9 y las Figuras 4.5 a 4.8 se muestran los resultados obtenidos.

Tabla 4.7. Análisis de cargas ESP (en unidades de |e|) y densidades de espín, entre paréntesis y en u.a., del dímero y los trímeros. Los átomos se numeran de izquierda a derecha.

\begin{tabular}{c|c|c|c|c} 
Agregado & B1 & B2 & N1 & N2 \\
\hline $\mathbf{( 1 )}$ & $0.33(1.12)$ & & $-0.33(0.89)$ & \\
\hline $\mathbf{( 2 )}$ & $-0.47(0.32)$ & & $0.80(0.14)$ & $-0.33(0.57)$ \\
\hline $\mathbf{( 3 )}$ & $0.63(-0.19)$ & & $-0.31(0.60)$ & $-0.31(0.60)$ \\
\hline $\mathbf{( 4 )}$ & $-0.13(0.49)$ & $-0.13(0.49)$ & $0.27(-0.01)$ & \\
\hline $\mathbf{( 5 )}$ & $0.24(1.26)$ & $0.17(0.43)$ & $-0.41(-0.67)$ &
\end{tabular}


Tabla 4.8. Análisis de cargas ESP (en unidades de |e|) y densidades de espín, entre paréntesis y en u.a., de los tetrámeros. Los átomos se numeran de izquierda a derecha, o como se muestra en la Figura 2.

\begin{tabular}{c|c|c|c|c|c|c} 
Agregado & $\mathrm{B} 1$ & $\mathrm{~B} 2$ & $\mathrm{~B} 3$ & $\mathrm{~N} 1$ & $\mathrm{~N} 2$ & $\mathrm{~N} 3$ \\
\hline $\mathbf{( 6 )}$ & $0.38(1.12)$ & $0.69(0.13)$ & & $-0.51(-0.04)$ & $-0.56(0.78)$ & \\
\hline $\mathbf{( 7 )}$ & $-0.19(0.44)$ & $-0.19(0.44)$ & & $0.19(0.55)$ & $0.19(0.55)$ & \\
\hline $\mathbf{( 8 )}$ & $0.61(0.46)$ & $0.61(0.46)$ & & $-0.61(0.55)$ & $-0.61(0.55)$ & \\
\hline $\mathbf{( 9 )}$ & -0.38 & 0.66 & -0.24 & -0.04 & & \\
\hline $\mathbf{( 1 0 )}$ & -0.28 & & & -0.02 & 0.58 & -0.28 \\
\hline $\mathbf{( 1 1 )}$ & $0.15(1.08)$ & $0.15(1.08)$ & & $-0.15(-0.08)$ & $-0.15(-0.08)$ & \\
\hline $\mathbf{( 1 2 )}$ & 0.15 & & & -0.47 & 0.44 & -0.11 \\
\hline $\mathbf{( 1 3 )}$ & $0.08(0.42)$ & $-0.33(0.57)$ & & $0.52(-0.08)$ & $-0.27(0.09)$ & \\
\hline $\mathbf{( 1 4 )}$ & $0.31(-0.16)$ & $0.31(-0.16)$ & & $-0.31(1.15)$ & $-0.31(1.15)$ & \\
\hline $\mathbf{( 1 5 )}$ & $0.18(1.44)$ & $0.06(0.31)$ & $0.18(-0.13)$ & $-0.41(0.38)$ & & \\
\hline $\mathbf{( 1 6 )}$ & $0.01(1.04)$ & $0.17(0.07)$ & & $-0.20(0.75)$ & $0.02(0.15)$ & \\
\hline $\mathbf{( 1 7 )}$ & 0.40 & 0.07 & & -0.10 & -0.36 &
\end{tabular}


Tabla 4.9. Análisis de cargas ESP (en unidades de $|e|$ ) y densidades de espín, entre paréntesis y en u.a., de los pentámeros. Los átomos se

\begin{tabular}{|c|c|c|c|c|c|c|c|c|}
\hline Agregado & B1 & B2 & B3 & B4 & N1 & N2 & $\mathrm{N} 3$ & $\mathrm{~N} 4$ \\
\hline (18) & $0.14(1.54)$ & $-0.03(1.03)$ & $0.06(-0.22)$ & $0.27(-0.21)$ & $-0.43(0.87)$ & & & \\
\hline (19) & $0.14(1.23)$ & $-0.16(0.35)$ & $-0.28(-0.28)$ & & $0.57(-0.01)$ & $-0.27(-0.30)$ & & \\
\hline (20) & $-0.57(0.17)$ & $1.55(0.38)$ & $1.56(0.38)$ & $-0.58(0.17)$ & $-1.96(-0.08)$ & & & \\
\hline (21) & $-0.10(1.96)$ & $0.12(0.85)$ & & & $-0.31(0.01)$ & $0.61(0.01)$ & $-0.32(0.17)$ & \\
\hline (22) & $-0.56(0.03)$ & $-0.21(0.02)$ & $0.19(1.19)$ & $0.03(1.68)$ & & $0.56(0.10)$ & & \\
\hline (23) & $0.25(0.91)$ & $0.20(0.01)$ & $0.16(0.04)$ & & $-0.14(-0.03)$ & $-0.47(0.06)$ & & \\
\hline (24) & $-0.18(0.53)$ & $-0.16(-0.01)$ & $-0.18(0.53)$ & & $0.26(-0.01)$ & $0.26(-0.01)$ & & \\
\hline (25) & $-0.52(0.03)$ & $-0.13(0.05)$ & & & $0.45(0.15)$ & $0.40(0.13)$ & $-0.20(0.63)$ & \\
\hline (26) & $-0.38(-0.25)$ & $-0.01(0.35)$ & $0.14(1.41)$ & & $0.37(-0.26)$ & $-0.12(-0.27)$ & & \\
\hline (27) & $0.18(1.39)$ & $0.56(-0.27)$ & & & $-0.02(-0.06)$ & $-0.28(0.56)$ & $-0.45(1.38)$ & \\
\hline (28) & $0.15(1.32)$ & $0.15(1.32)$ & & & $-0.08(-0.09)$ & $-0.15(0.55)$ & $-0.08(-0.09)$ & \\
\hline (29) & $0.17(1.12)$ & & & & $-0.19(-0.11)$ & $-0.26(0.01)$ & $0.55(0.00)$ & $-0.27(-0.02)$ \\
\hline (30) & $0.37(-0.01)$ & $0.06(-0.40)$ & $0.37(-0.01)$ & & $-0.40(0.71)$ & $-0.40(0.71)$ & & \\
\hline (31) & $0.22(-0.14)$ & $-0.04(0.24$ & & & $-0.44(0.33)$ & $0.45(0.04)$ & $-0.18(0.51)$ & \\
\hline (32) & $0.69(0.18)$ & $0.26(0.60)$ & $0.08(1.66)$ & & $-0.51(-1.17)$ & $-0.52(-0.28)$ & & \\
\hline (33) & $0.91(-0.06)$ & $0.91(-0.06)$ & & & $-0.46(1.34)$ & $-0.90(0.41)$ & $-0.46(1.34)$ & \\
\hline (34) & $0.58(0.07)$ & & & & $-0.52(0.81)$ & $-0.37(0.05)$ & $0.53(-0.02)$ & $-0.22(0.08)$ \\
\hline (35) & $-0.49(0.46)$ & & & & $-0.27(0.34)$ & $0.51(-0.07)$ & $0.51(-0.07)$ & $-0.27(0.34)$ \\
\hline (36) & $0.23(0.92)$ & $0.88(0.04)$ & $-0.29(0.02)$ & & $-0.41(0.01)$ & $-0.41(0.01)$ & & \\
\hline (37) & $0.25(0.95)$ & $0.82(0.05)$ & & & $-0.36(0.10)$ & $-0.36(0.10)$ & $-0.34(1.81)$ & \\
\hline (38) & $0.55(0.06)$ & $0.06(0.13)$ & & & $-0.15(0.70)$ & $-0.17(0.37)$ & $-0.29(1.75)$ & \\
\hline (39) & $0.09(0.42)$ & $0.14(-0.10)$ & $0.08(1.57)$ & & $-0.22(0.64)$ & $-0.10(0.45)$ & & \\
\hline (40) & $0.21(1.39)$ & $-0.08(1.16)$ & $0.21(1.41)$ & & $-0.17(0.08)$ & $-0.16(0.97)$ & & \\
\hline (41) & $0.58(-0.12)$ & $1.26(-0.09)$ & $-0.36(0.01)$ & & $-0,74(0.60)$ & $-0.74(0.60)$ & & \\
\hline (42) & $0.02(0.99)$ & $0.02(0.99)$ & $-0.13(1.98)$ & & $-0.16(0.80)$ & $0.26(0.23)$ & & \\
\hline (43) & $0.14(1.06)$ & $0.14(1.06)$ & & & $-0.26(0.97)$ & $0.27(0.16)$ & $-0.29(1.78)$ & \\
\hline (44) & $0.73(-0.07)$ & $-0.94(-0.07)$ & & & $-0.66(0.68)$ & $-0.66(0.68)$ & $-0.35(0.02)$ & \\
\hline (45) & $-0.07(1.16)$ & $0.20(1.38)$ & $0.21(1.41)$ & & $-0.16(0.97)$ & $-0.19(0.08)$ & & \\
\hline
\end{tabular}


(1)

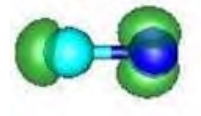

(4)
(2)

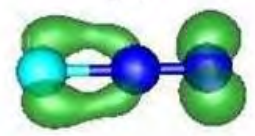

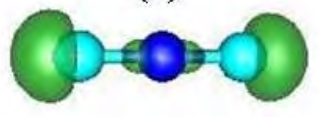

(3)

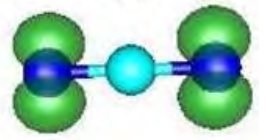

(5)

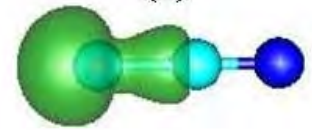

Figura 4.5. Densidades de espín del dímero y los trímeros. Las superficies se graficaron con un valor de isosuperficie de 0.015 u.a.

(6)

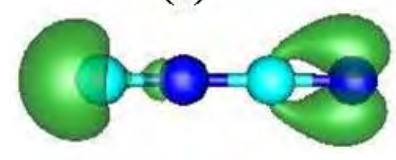

(13)

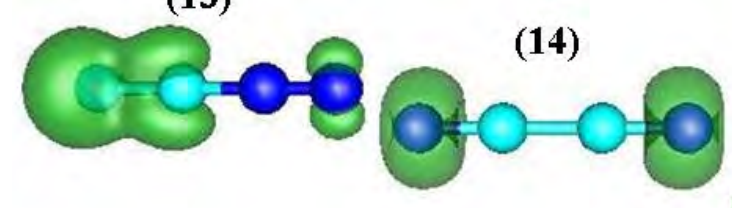

(8)

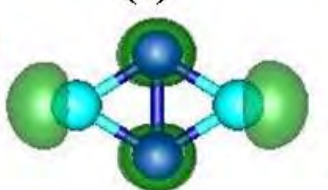

(14)

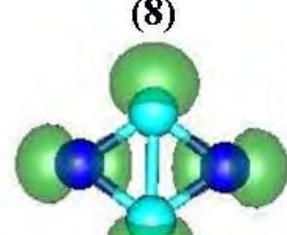

(

(15)
(11)

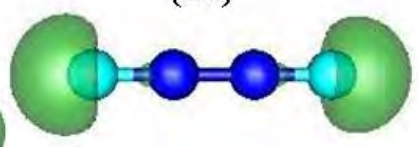

(16)

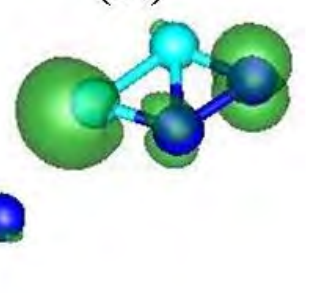

Figura 4.6. Densidades de espín de los tetrámeros de capa abierta. Las superficies se graficaron con un valor de isosuperficie de 0.015 u.a. 
(18)

(19)

(20)

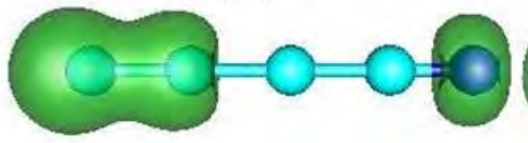

(21)

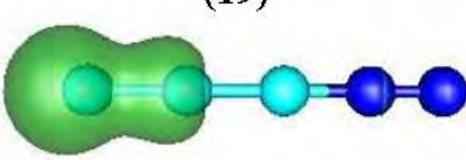

(22)

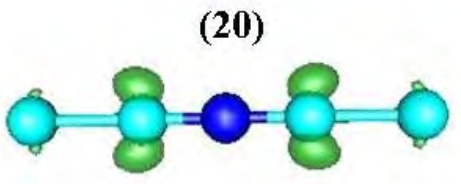

(23)

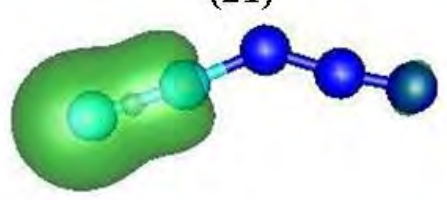

(24)

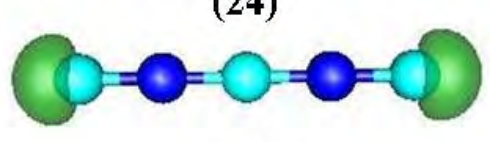

(27)
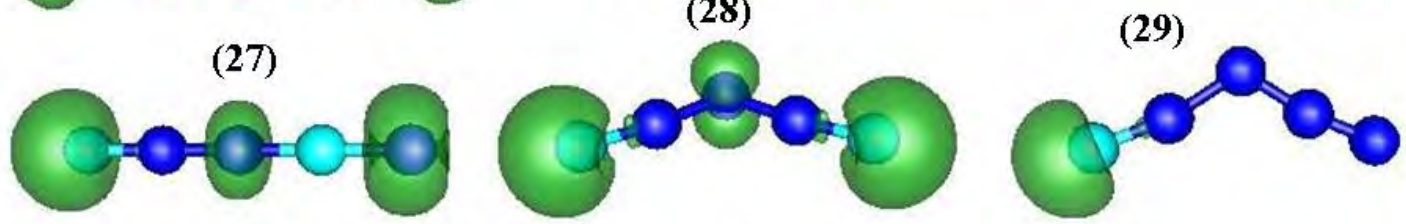

(30)
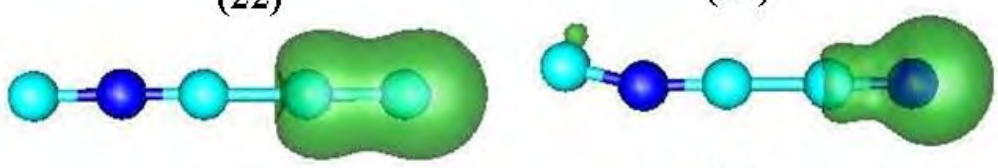

(25)

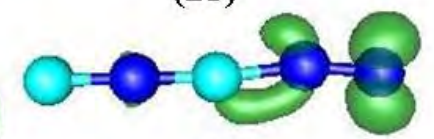

(28)

(26)

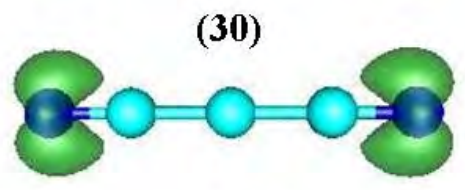

(31)

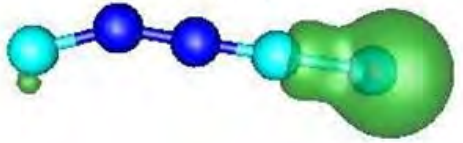

(29)

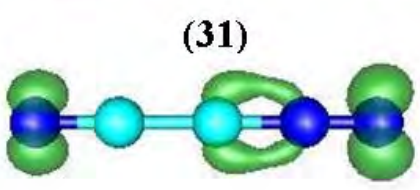

(32)

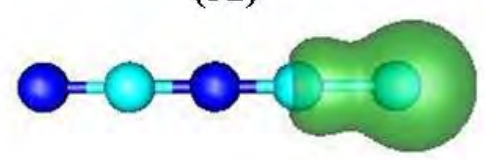

(33)

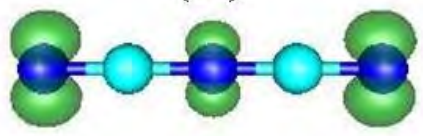

Figura 4.7. Densidades de espín de pentámeros (18) a (33). Las superficies se graficaron con un valor de isosuperficie de 0.015 u.a. 
(34)

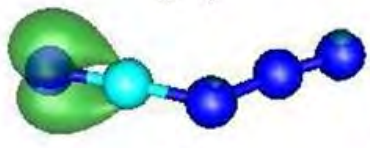

(38)

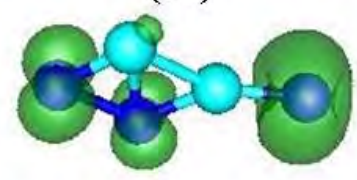

(42)

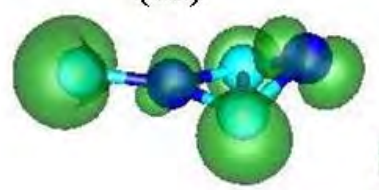

(35)

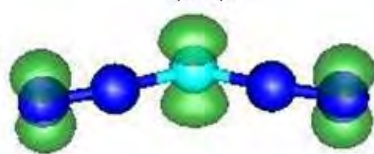

(39)

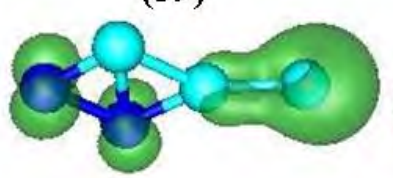

(43)

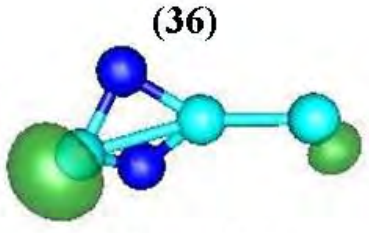

(40)

(45)

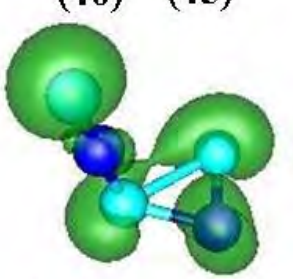

(44)
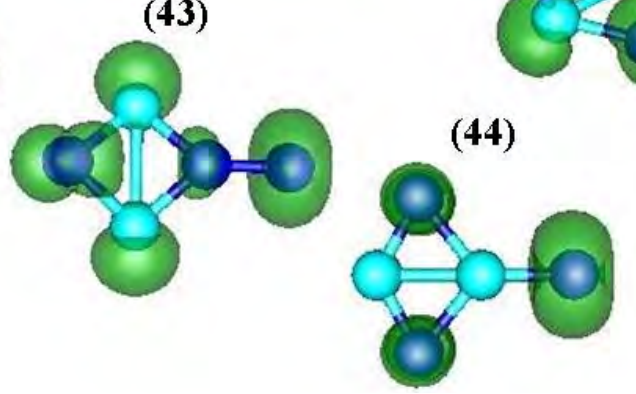

(37)

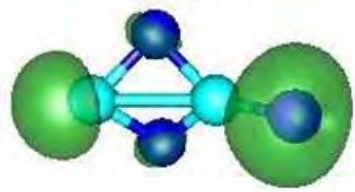

(41)

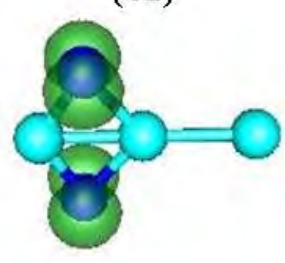

(34) a (45). Las superficies se graficaron con

Figura 4.8. Densidades de espín de pentámeros (34) a (45). Las supe
un valor de isosuperficie de 0.015 u.a.

\subsection{Potenciales electrostáticos moleculares}

En las Figuras 4.9 a 4.13 se muestran las representaciones gráficas de los potenciales electrostáticos moleculares calculados para todos los sistemas. El color azul indica una zona susceptible de ataque electrofílico y el color rojo, una zona probable de ataque nucleofílico; mientras que los colores amarillos y verdes indican zonas intermedias de valores muy bajos.

(1)

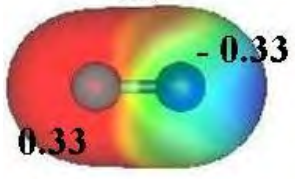

(2)

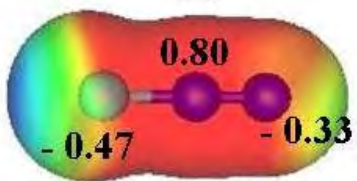

(3)

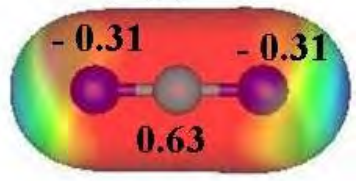

(4)

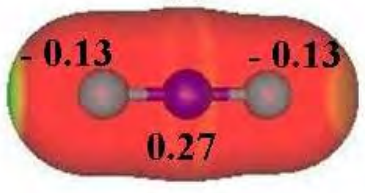

(5)

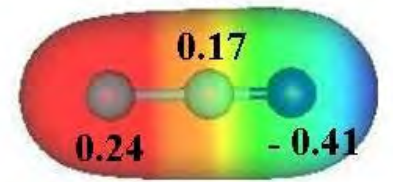

Figura 4.9. Superficies de potenciales electrostáticos moleculares del dímero y los trímeros. También se muestran las cargas ESP calculadas. Las superficies se graficaron con un valor de isosuperficie de la densidad electrónica de 0.01 u.a.

En el dímero la zona susceptible de ataque electrofílico se encuentra alrededor del átomo de nitrógeno. A medida que el sistema crece y se agregan átomos, el comportamiento es diferente. En el trímero (4), el de mayor $E_{a t}$ de la serie, la zona susceptible de ataque electrofílico se encuentra sobre los átomos de boro, de manera similar al cluster (2), que le 
sigue en orden decreciente de $\mathrm{E}_{\mathrm{at}}$. En el agregado (5), la zona susceptible de ataque electrofílico se concentra en el átomo de nitrógeno, al igual que en el agregado (3). Por lo anteriormente expresado, se evidencia que, en el caso de los trímeros, es importante para el crecimiento que la zona susceptible de ataque electrofílico se sitúe en átomos de boro.

Con respecto a los resultados obtenidos para la densidad de espín de trímeros y tetrámeros (Tabla 4.7 y Figura 4.5), se observa que se encuentran electrones desapareados en las zonas de MEP negativo, susceptibles a ataque electrofílico, pero esto no es importante para el crecimiento de los clusters, dado que no se evidencia una tendencia paralela al aumento de la $\mathrm{E}_{\mathrm{at}}$.

(6)

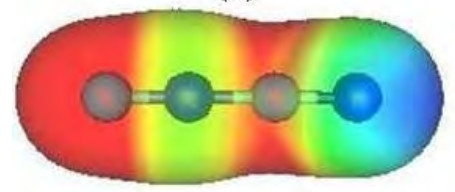

(9)

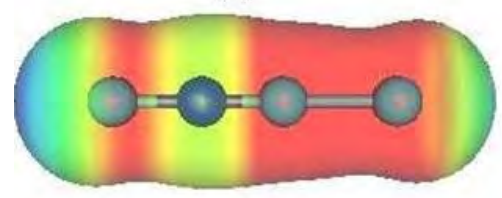

(12)

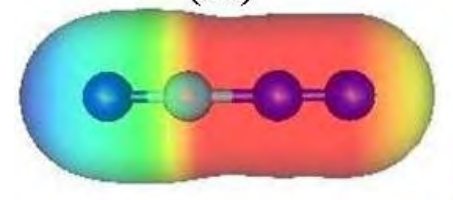

(15)

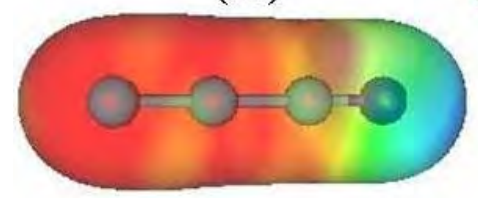

(7)

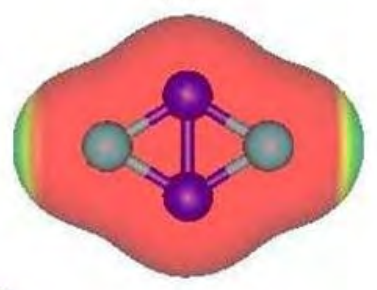

(10)
(8)

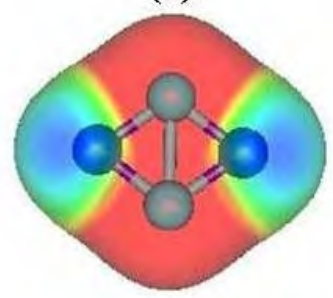

(11)

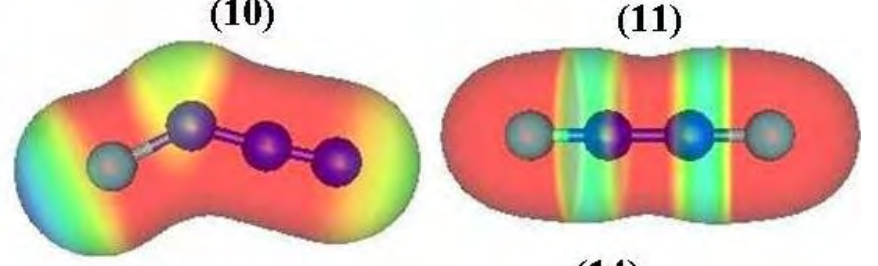

(14)
(13)

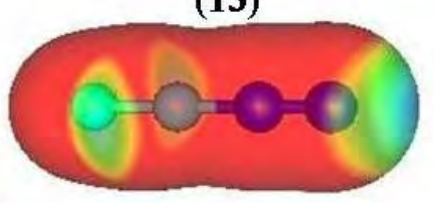

(16)

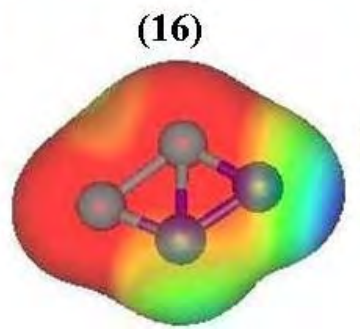

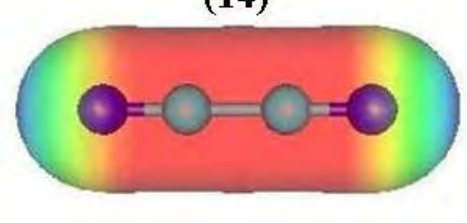

(17)

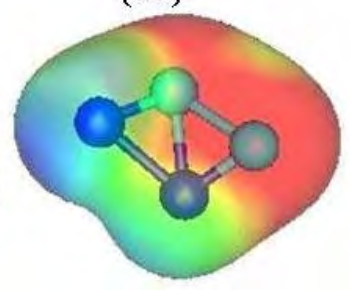

Figura 4.10. Superficies de potenciales electrostáticos moleculares para los tetrámeros. Las superficies se graficaron con un valor de isosuperficie de la densidad electrónica de 0.01 u.a.

Con respecto a los tetrámeros, vemos en la Figura 4.10 que las zonas susceptibles de ataque electrofílico se encuentran sobre los átomos de nitrógeno, con excepción de los agregados (7), (9) y (10). El agregado (9) concentra el potencial electrostático negativo alrededor de átomos de boro. El caso (7), el agregado BNBN rombo, se puede comparar con (8), que también resultó ser un rombo de diferentes dimensiones aunque mucho más estable, por una diferencia de $0.44 \mathrm{eV} / a ́ t$. Comparando estos dos casos y los dos clusters más estables (12) y (6) con (10), se ve que en el caso de los tetrámeros es importante para la estabilidad, y para el aumento de la $\mathrm{E}_{\mathrm{at}}$, que la zona de MEP negativo se encuentre sobre átomos de nitrógeno, condicionando el crecimiento. 
(18)

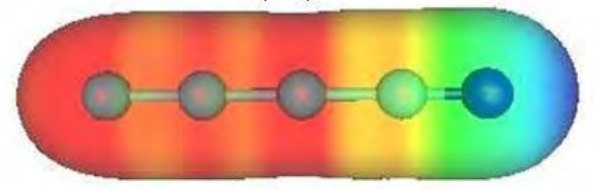

(20)

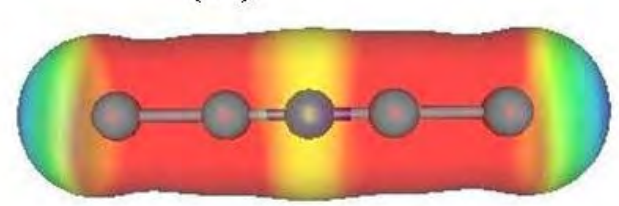

(22)

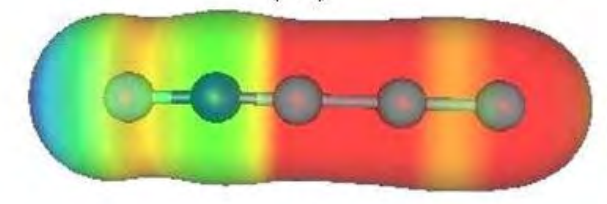

(24)
(19)

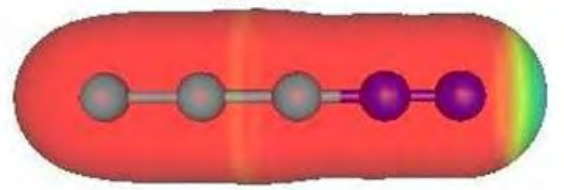

(21)

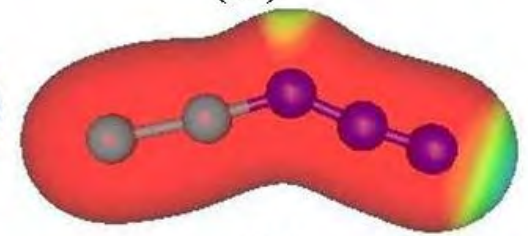

(23)

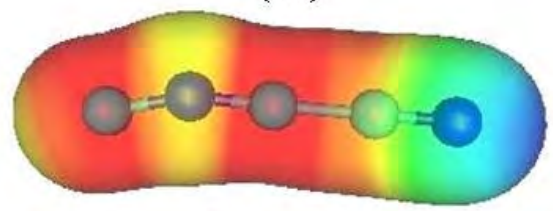

(25)

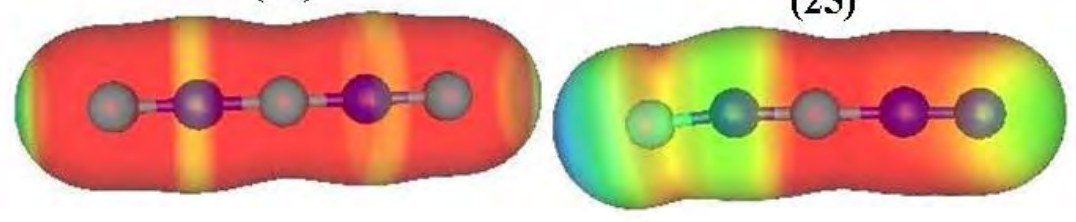

Figura 4.11. Superficies de potenciales electrostáticos moleculares para los pentámeros (18) a (25). Las superficies se graficaron con un valor de isosuperficie de la densidad electrónica de 0.01 u.a. 
(26)

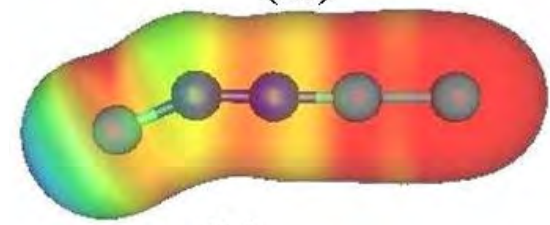

(28)

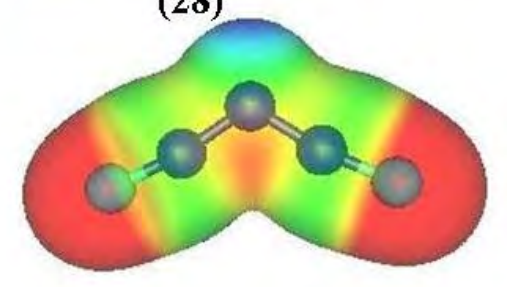

(30)

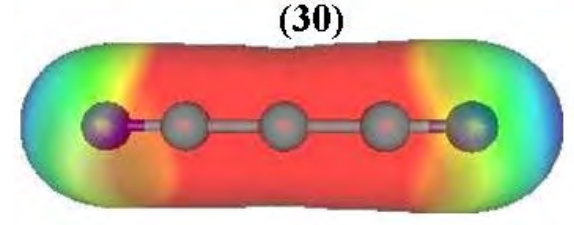

(32)

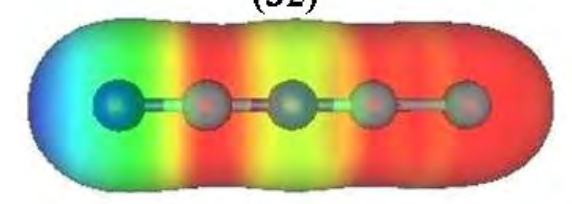

(27)
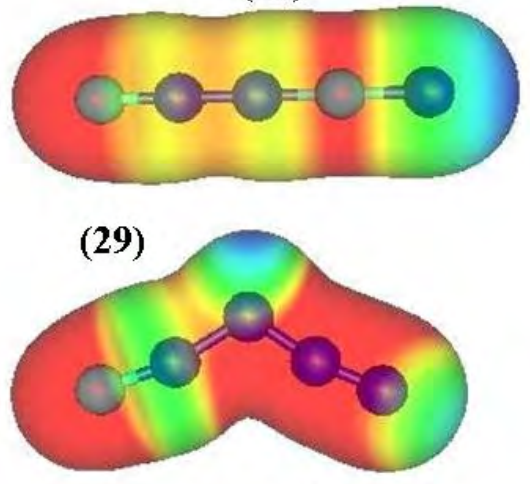

(31)

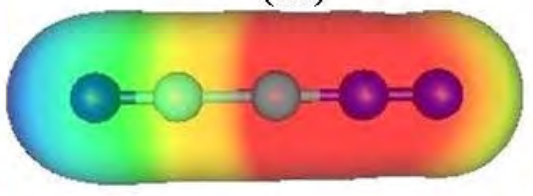

(33)

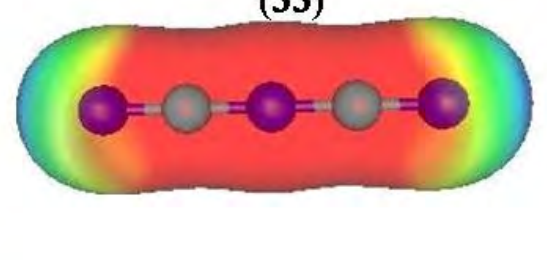

Figura 4.12. Superficies de potenciales electrostáticos moleculares para los pentámeros (25) a (33). Las superficies se graficaron con un valor de isosuperficie de la densidad electrónica de 0.01 u.a.

De las Figuras 4.11 y 4.12 se desprende que los agregados de cinco átomos que poseen átomos de nitrógeno en sus extremos o ángulos pronunciados (agregados (28) y (29)), sitúan las zonas susceptibles de ataque electrofílico en ellos. Asimismo, se observa que los agregados que poseen mayoría de átomos de boro, como (20) y (22), poseen zonas susceptibles de ataque electrofílico en átomos de boro terminales. 


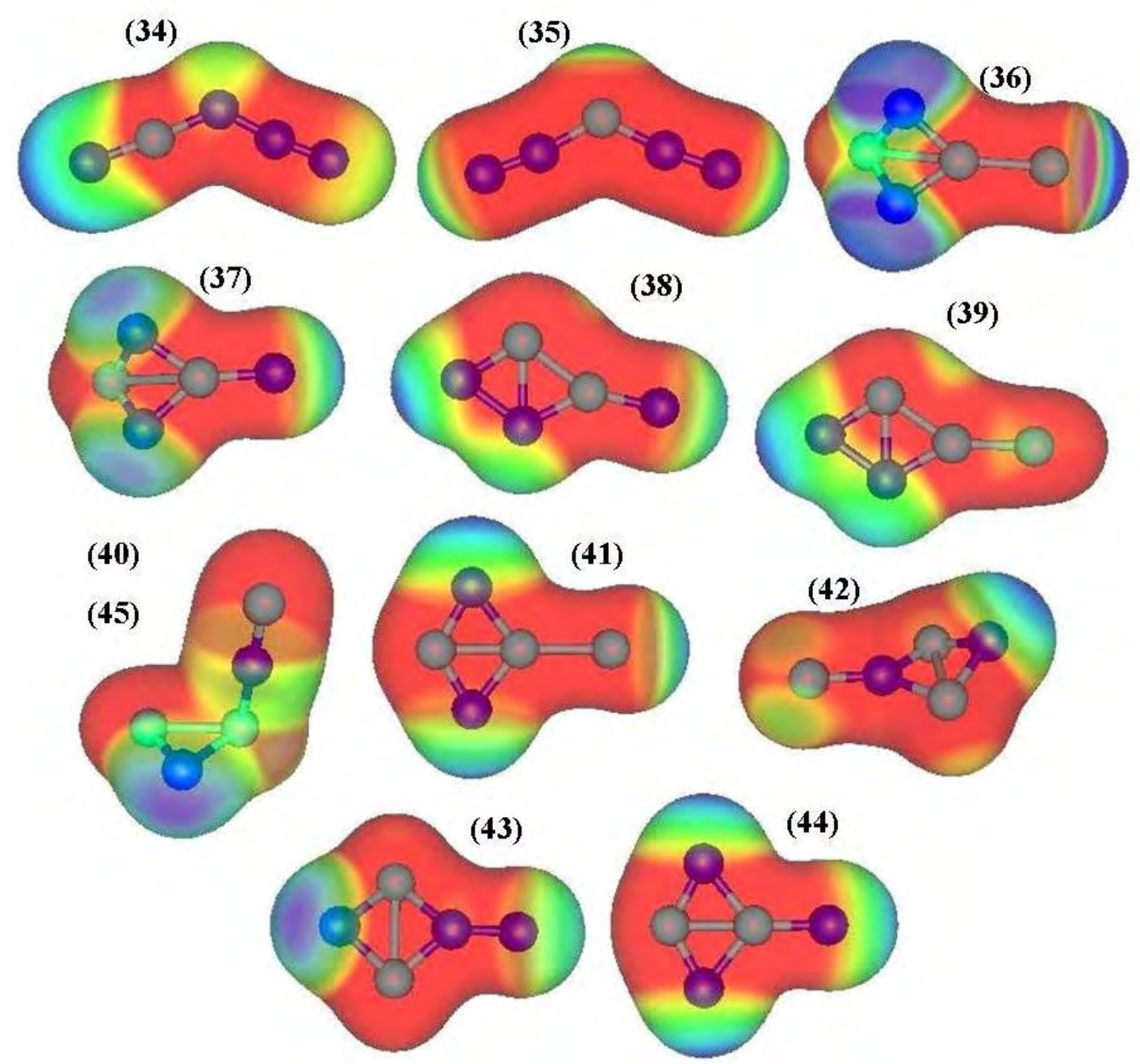

Figura 4.13. Superficies de potenciales electrostáticos moleculares para los pentámeros (34) a (45). Las superficies se graficaron con un valor de isosuperficie de la densidad electrónica de 0.01 u.a.

Los pentámeros de la Figura 4.13, siguen la tendencia mostrada en la figura anterior, átomos de nitrógeno en extremos o ángulos pronunciados concentran las zonas de ataque electrofílico, aún si hay un átomo de boro en otro extremo, con algunas excepciones. Un caso interesante es el del agregado (41), que distribuye las zonas de ataque electrofílico entre los dos nitrógenos del rombo y el boro del extremo.

Aún así, el comportamiento con respecto al aumento de la energía de atomización, y por lo tanto, del crecimiento, cambia en el caso de los agregados de cinco átomos con respecto a los agregados de cuatro átomos. Se observa en la Figura 4.11 que los agregados (24) y (25), los más estables de la serie (Tabla 4.5), concentran las zonas de MEP negativo en los átomos de boro en sus extremos, de manera similar a los agregados de tres átomos (Tabla 4.7 y Figura 4.9).

En el caso de los pentámeros, las zonas susceptibles de ataque electrofílico no siempre presentan diferencias en la densidad de espín (Tabla 4.9 y Figuras 4.7 y 4.8), que muestra la evidencia de electrones desapareados. Es decir, las zonas de ataque electrofílico pueden estar conformadas por pares libres, en algunos casos. 


\subsection{Patrones de crecimiento}

A continuación se muestran los patrones de crecimiento obtenidos. Estos patrones se construyen a partir del dímero (1) agregando átomos de $\mathrm{N}$ y de $\mathrm{B}$, siguiendo el camino de aumento de la energía de atomización, como se expresó con anterioridad. Se plantea el patrón de crecimiento más probable para agregados binarios constituidos por $\mathrm{B}$ y $\mathrm{N}$.

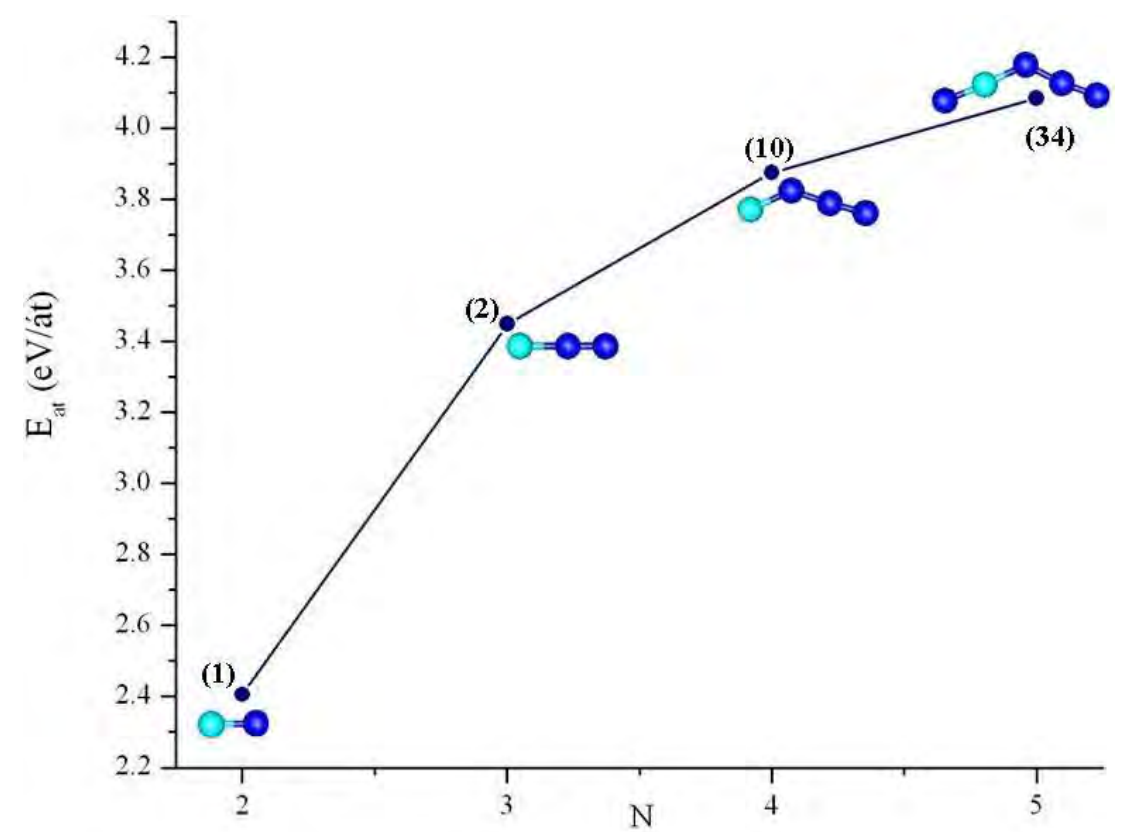

Figura 4.14.a Patrón de crecimiento del trímero (2) y el tetrámero (10).

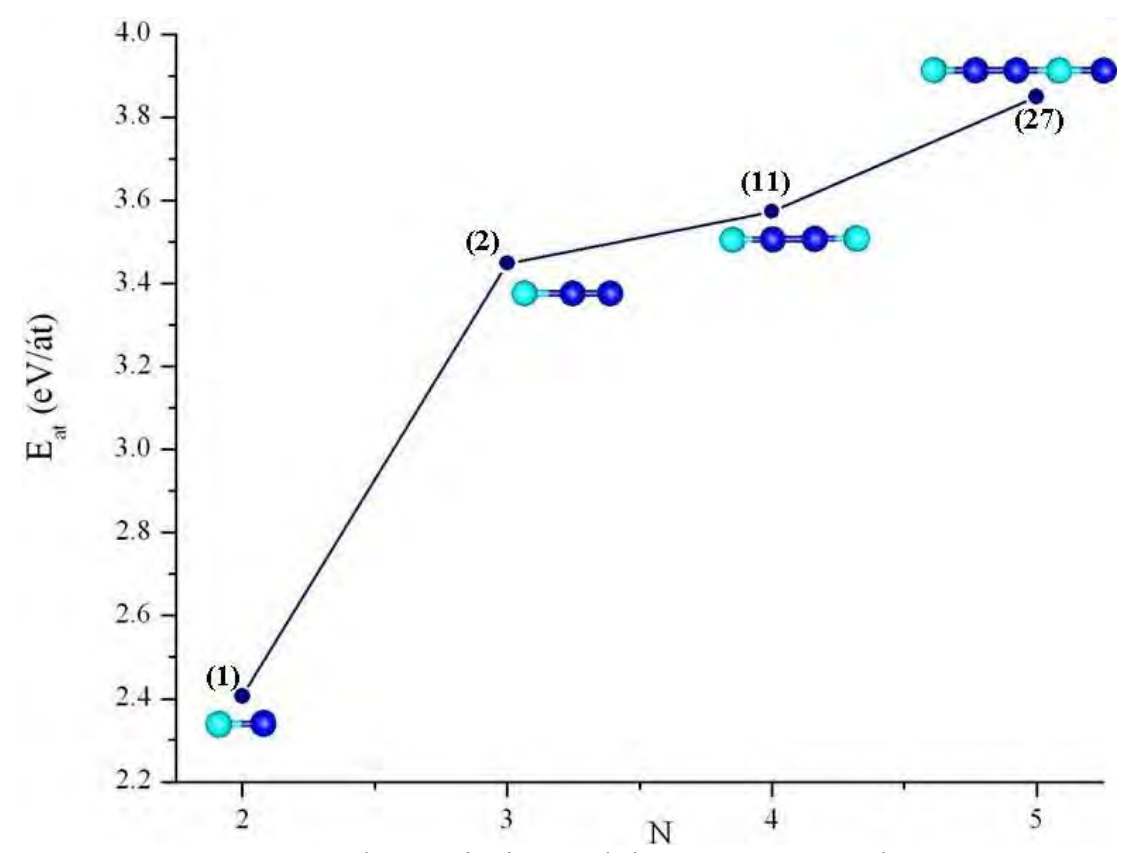

Figura 4.14.b Patrón de crecimiento del trímero (2) y el tetrámero (11).

En las Figuras 4.14.a y 4.14.b se muestran los patrones de crecimiento del trímero (2) con los tetrámeros (10) y (11), respectivamente. Considerando el crecimiento desde el dímero (2), se 
observa que es más favorable para el sistema unir un átomo de $\mathrm{N}$ al nitrógeno terminal con carga ESP negativa de menor valor que la carga de B1 (Tabla 4.7), para formar (10), que unir un átomo de B para formar (11).

Con respecto a la Figura 4.14.a, se ve que para formar el pentámero el camino de aumento de la $E_{a t}$ se ve favorecido por enlazar un $\mathrm{N}$ a B1, que posee menor carga ESP que N3 en (10). Es importante aclarar que en la Tabla 4.8 los valores se expresan con dos decimales. Los valores de cargas ESP de B1 y N3 en (10) son $-0.279|\mathrm{e}|$ y -0.282 |e|, respectivamente.

Observando la Figura 4.14.b junto con la Tabla 4.8, se evidencia que el pentámero se forma por la unión de un átomo de nitrógeno en una zona susceptible de ataque nucleofílico, pero con electrones desapareados (Figura 4.6).

Otros crecimientos encontrados para el trímero (2) se muestran en conjunto con otros patrones en las Figuras 4.17.a, 4.17.c y 4.17.d.

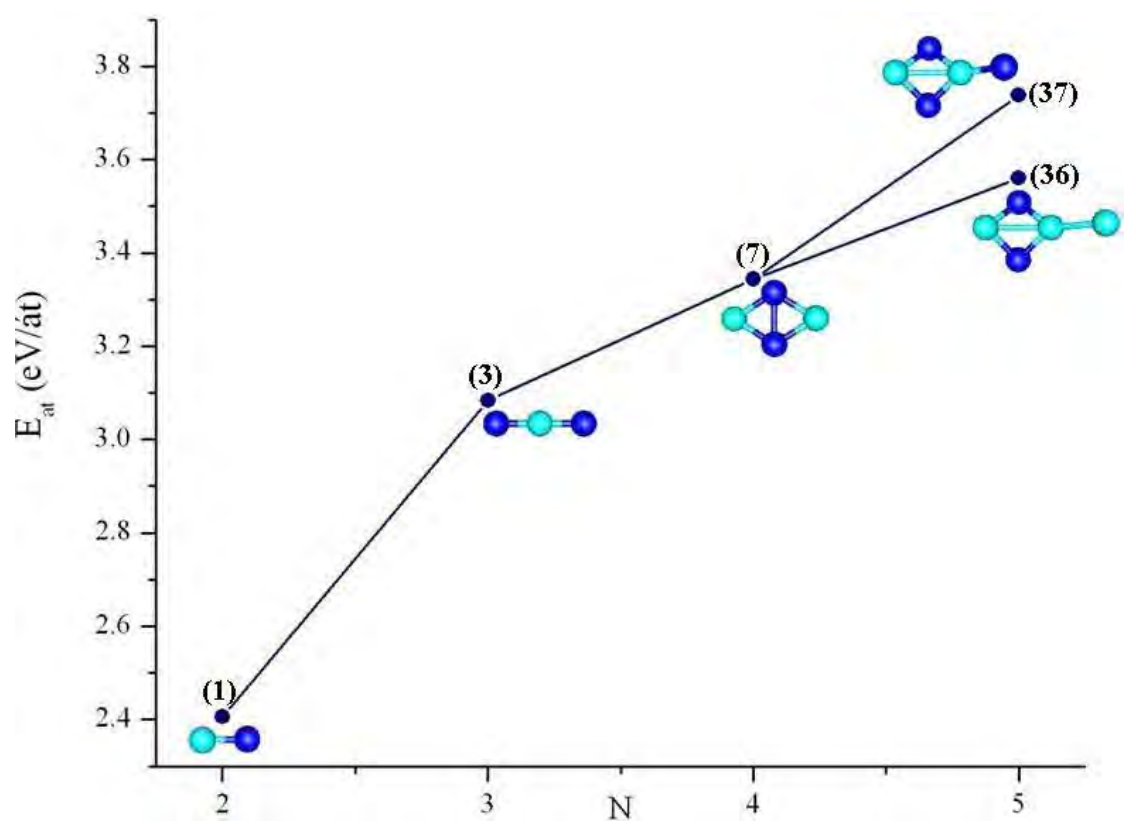

Figura 4.15.a Patrón de crecimiento del trímero (3) y el tetrámero (7). 


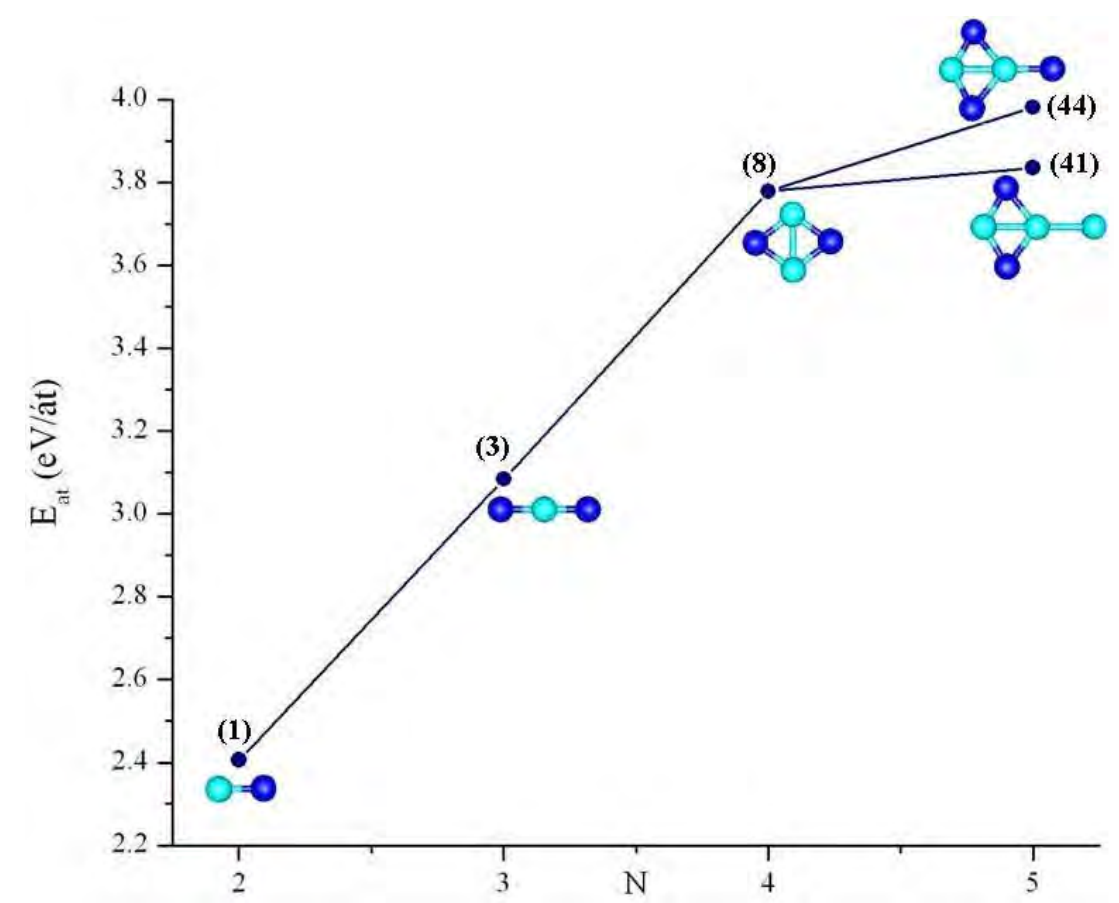

Figura 4.15.b Patrón de crecimiento del trímero (3) y el tetrámero (8).

En las Figuras 4.15.a y 4.15.b se muestran los patrones de crecimiento obtenidos para el trímero (3). El tetrámero (7) posee zonas de ataque electrofílico, cargas ESP negativas, sobre los átomos de boro (Tabla 4.8 y Figura 4.10), encontrándose electrones desapareados en sus cuatro átomos (Figura 4.6). Al continuar el crecimiento y formar el pentámero, más favorecido es (37) sobre (36), que se forma uniendo un átomo de $\mathrm{N}$ a un átomo de $\mathrm{B}$ del tetrámero.

El tetrámero (8), por otro lado, que presenta el patrón de crecimiento mostrado en la Figura 4.14.b, posee cargas ESP negativas en átomos de nitrógeno. Aún así, el pentámero más favorecido para su crecimiento es el que se forma uniendo un átomo de $\mathrm{N}$ al átomo de boro del tetrámero, (44).

Otros patrones de crecimiento para este trímero en conjunto con otros trímeros se muestran en las Figuras 4.17.a y 4.17.b. 


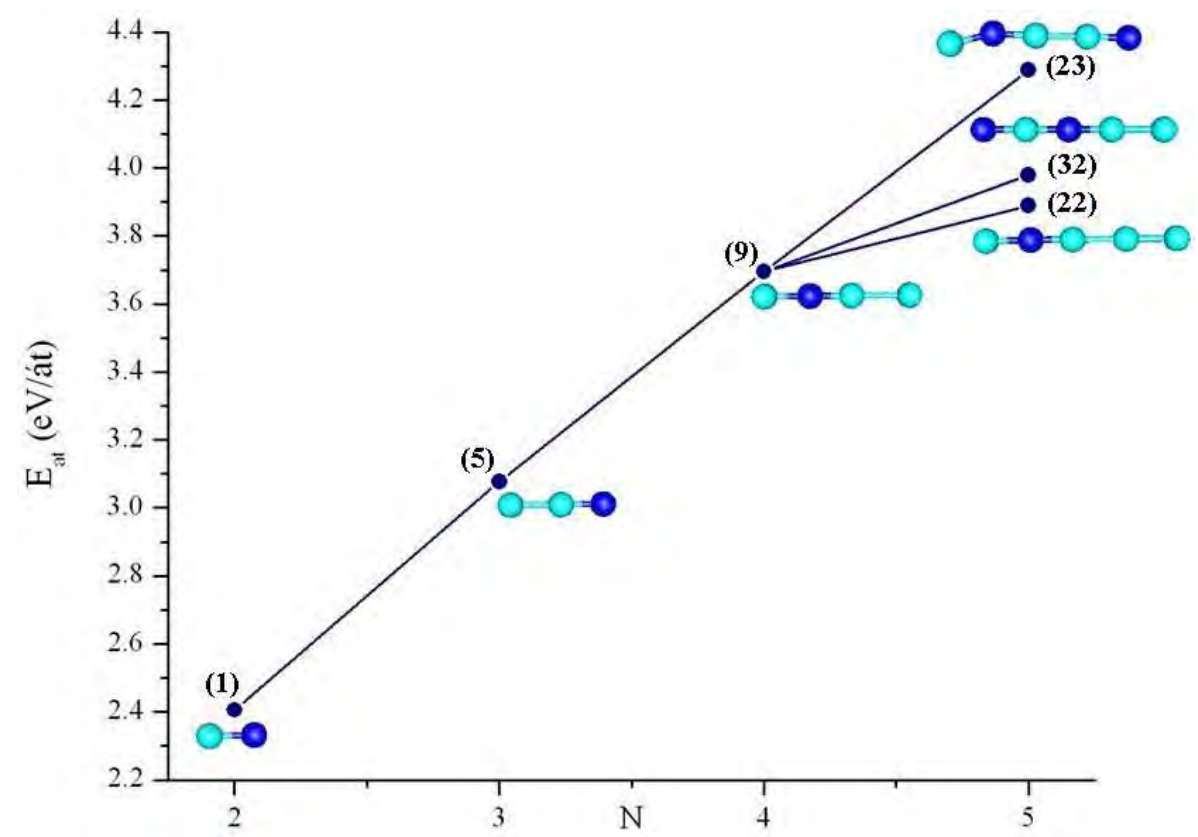

Figura 4.16.a Patrón de crecimiento del trímero (5) y el tetrámero (9).

En la Figura 4.16.a se encuentra el patrón de crecimiento del trímero (5) y el tetrámero (9). Se observa, teniendo en cuenta la Tabla 4.7 y las Figuras 4.5 y 4.9, que para formar el tetrámero (9), un átomo de B ataca una zona susceptible de ataque nucleofilico que no posee electrones desapareados en el trímero (5). Por otro lado, la formación del pentámero a partir de este tetrámero se ve favorecida si un átomo de nitrógeno ataca una zona susceptible de ataque electrofílico como es el B3. Asimismo, el pentámero (32) se forma por un nitrógeno que ataca una zona susceptible de ataque electrofílico en el átomo B1, con carga ESP más negativa que $\mathrm{B} 3$, pero su $\mathrm{E}_{\mathrm{at}}$ resulta menor. El pentámero formado mediante el ataque de un átomo de boro al B3, (22), resulta de menor $\mathrm{E}_{\mathrm{at}}$. 


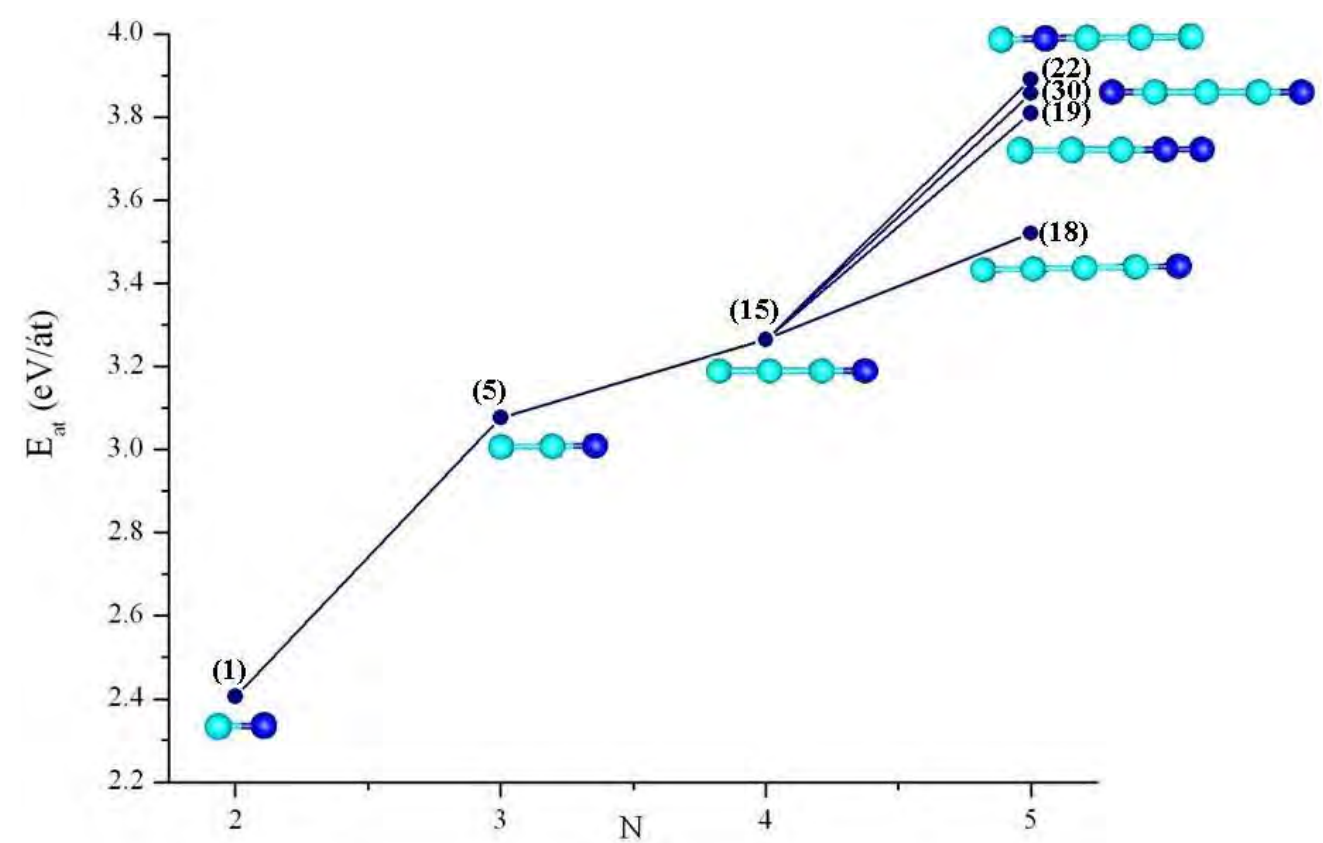

Figura 4.16.b Patrón de crecimiento del trímero (5) y el tetrámero (15).

Con respecto al patrón de crecimiento de (5) y (15), se ve que (15) se forma por el ataque de un átomo de B a una zona susceptible de ataque nucleofílico de (5), B1, con electrones desapareados (Tabla 4.7, Figura 4.5 y 4.9). Para la formación del pentámero se ve más favorecida la unión de un átomo de $\mathrm{B}$ en el N1 del tetrámero, de carga ESP negativa, que la de un átomo de $\mathrm{N}$ a la zona de carga ESP positiva, como en (30) y más aún que si un átomo de nitrógeno ataca en la zona de carga ESP negativa y se une a N1, como en (19). El pentámero menos probable de obtener por este crecimiento es (18), donde un átomo de $\mathrm{B}$ ataca la zona susceptible de ataque nucleofílico en B1 de (15).

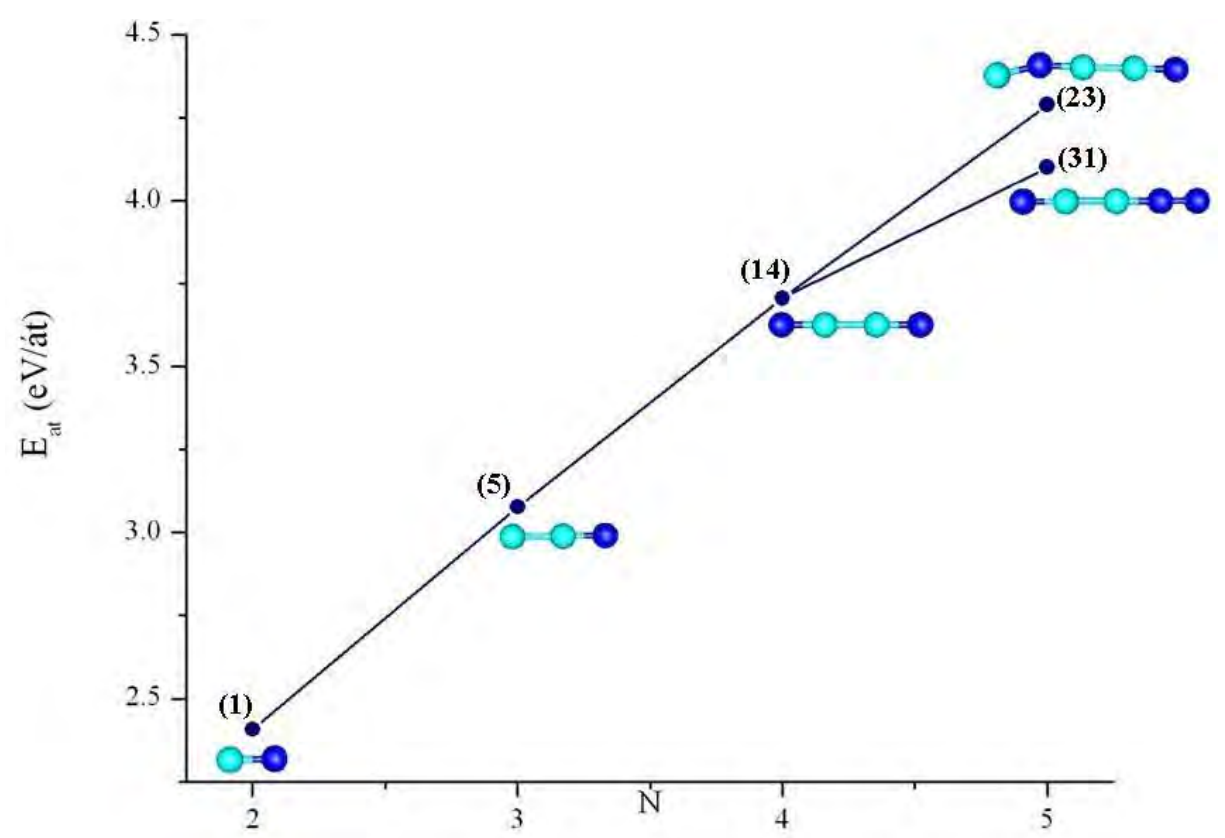

Figura 4.16.c Patrón de crecimiento del trímero (5) y el tetrámero (14). 
Con respecto al patrón de crecimiento de (5) y (14), el tetrámero se forma mediante un átomo de $\mathrm{N}$ que ataca una zona susceptible de ataque nucleofílico, B1 (Tabla 4.7). Luego, el pentámero (23), de mayor $\mathrm{E}_{\mathrm{at}}$ que (31), se forma uniendo un átomo de boro al $\mathrm{N}$ de carga ESP negativa y con electrones desapareados (Tabla 4.8, Figuras 4.6 y 4.10).

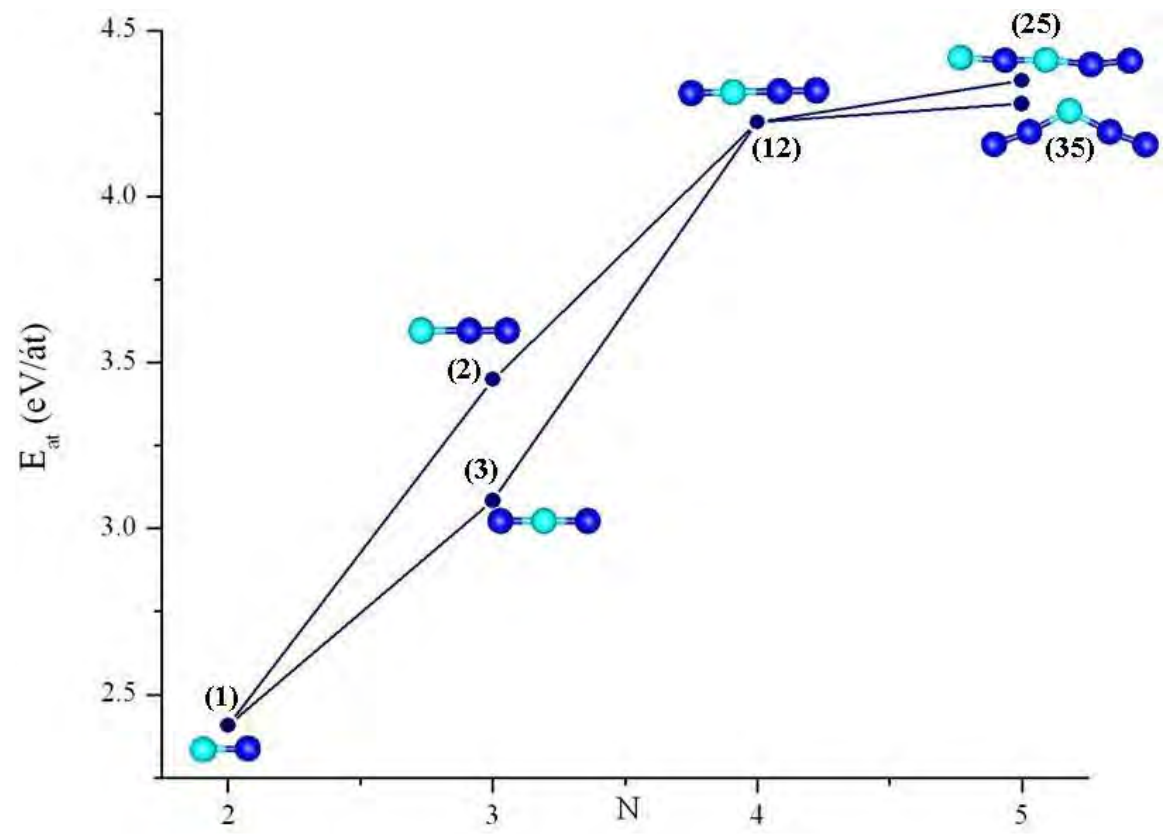

Figura 4.17.a Patrón de crecimiento del trímero (2) con trímero (3) y el tetrámero (12).

En la Figura 4.17.a se muestra el patrón de crecimiento de los trímeros (2) y (3) con el tetrámero (12). Cualquier camino es válido para obtener (12), aunque el camino a partir de (2) se ve más favorecido. Para formar (12) a partir de (3), un átomo de nitrógeno ataca en el nitrógeno de (3), de carga ESP negativa (Tabla 4.7) y con electrones desapareados (Figura 4.5). Por otro lado, para formar (12) a partir de (2), un átomo de nitrógeno deberá atacar en la zona de carga ESP más negativa, B1, que también es más negativa que la carga ESP del nitrógeno en (3). Al formar el pentámero a partir de (12), es más favorable unir un átomo de boro a N1, con una carga ESP de -0.47 |e| (Tabla 4. 8), que unir un átomo de nitrógeno en el mismo sitio. 


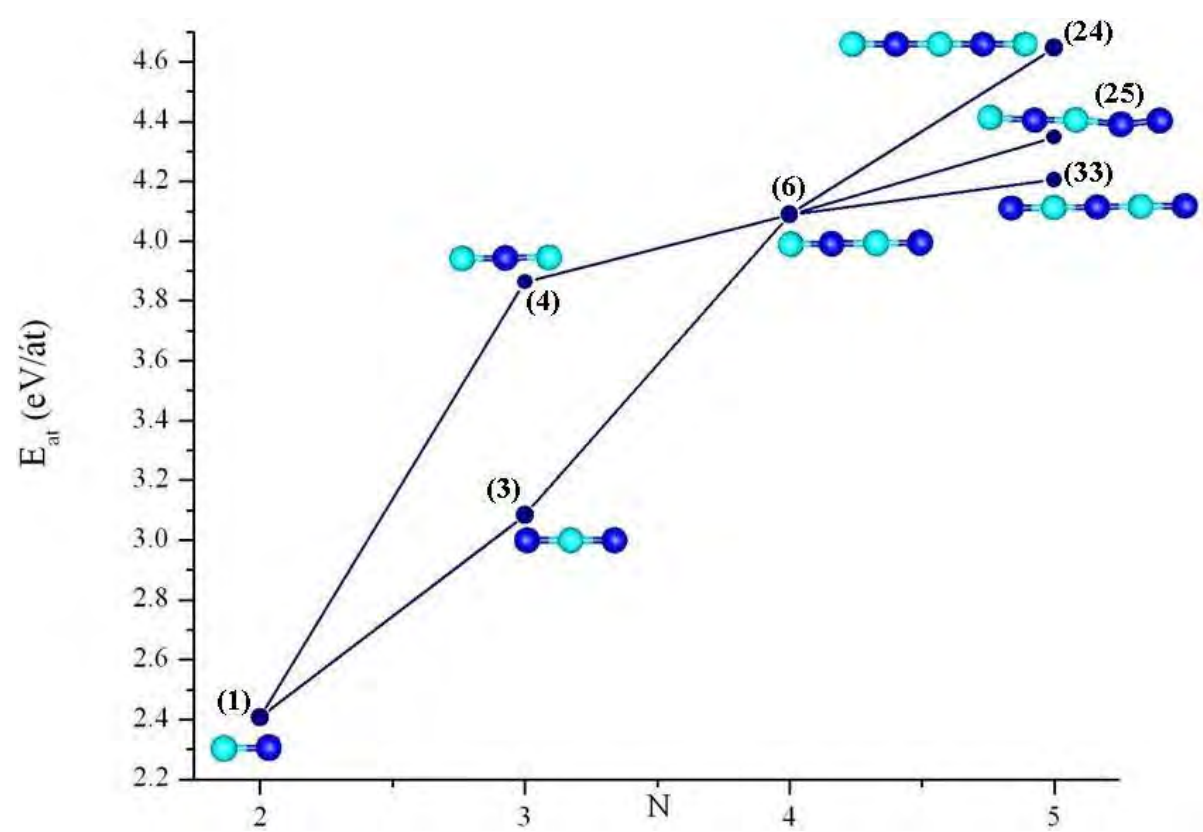

Figura 4.17.b Patrón de crecimiento del trímero (3) con trímero (4) y el tetrámero (6).

Por otro lado, en el patrón mostrado en la Figura 4.16.b, se ve que el crecimiento del tetrámero (6) se ve más favorecido si se forma a partir de (4) que de (3). Para formarse a partir de cualquiera de los dos trímeros un átomo de boro tiene que atacar en una zona susceptible de ataque electrofílico (Tabla 4.7), pero se favorece la que menor carga ESP posee, los átomos de boro de (4). Para formar el pentámero a partir de (6), se ve favorecido el ataque de un átomo de boro a un átomo de nitrógeno susceptible de ataque electrofílico (Tabla 4.8) y se forma (24), siendo menos probable la obtención de (25), donde un átomo de nitrógeno se une a la zona de ataque electrofílico o la obtención de (33), donde el átomo de nitrógeno se une a B1, una zona susceptible de ataque nucleofílico.

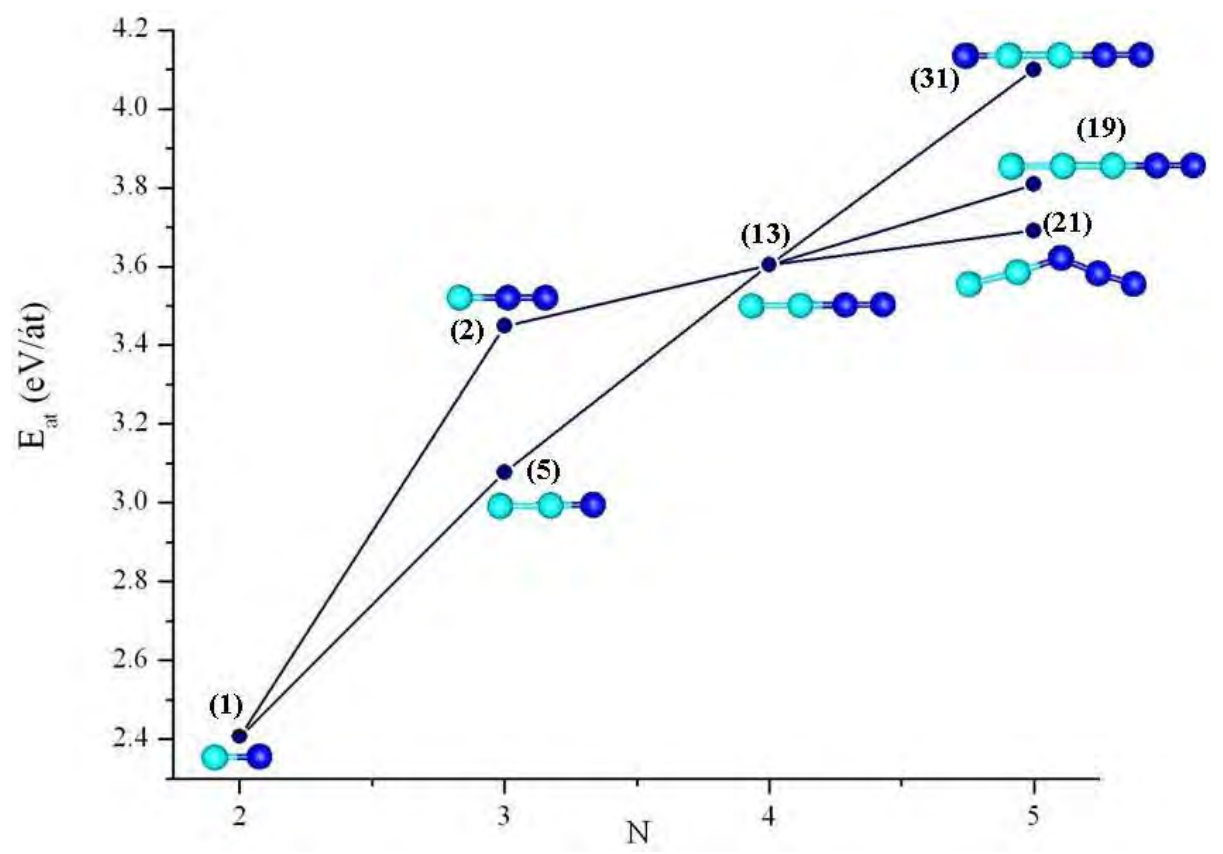

Figura 4.17.c Patrón de crecimiento del trímero (2), con trímero (5) y el tetrámero (13). 
La obtención del tetrámero (13), Figura 4.16.c, se ve favorecida si es a partir del trímero (2), para ello un átomo de boro tiene que unirse a B1 de (2), que posee una carga ESP más negativa que N2 (Tabla 4.7). Si se formara a partir de (5), debería unirse un átomo de nitrógeno a la zona susceptible de ataque electrófilico, N1, aunque esto es menos probable. A partir de este tetrámero se obtienen tres pentámeros, siendo más probable el crecimiento de (31). Para formar (31) un átomo de $\mathrm{N}$ ataca una zona susceptible de ataque nucleofílico de carga ESP positiva muy pequeña, 0.08 |e $\mid$ (Tabla 4.8), ataque más probable que el de un átomo de $\mathrm{B}$ en el mismo B1 de (13) para formar (19), o de un átomo de nitrógeno en N2 de (13) para formar (21).

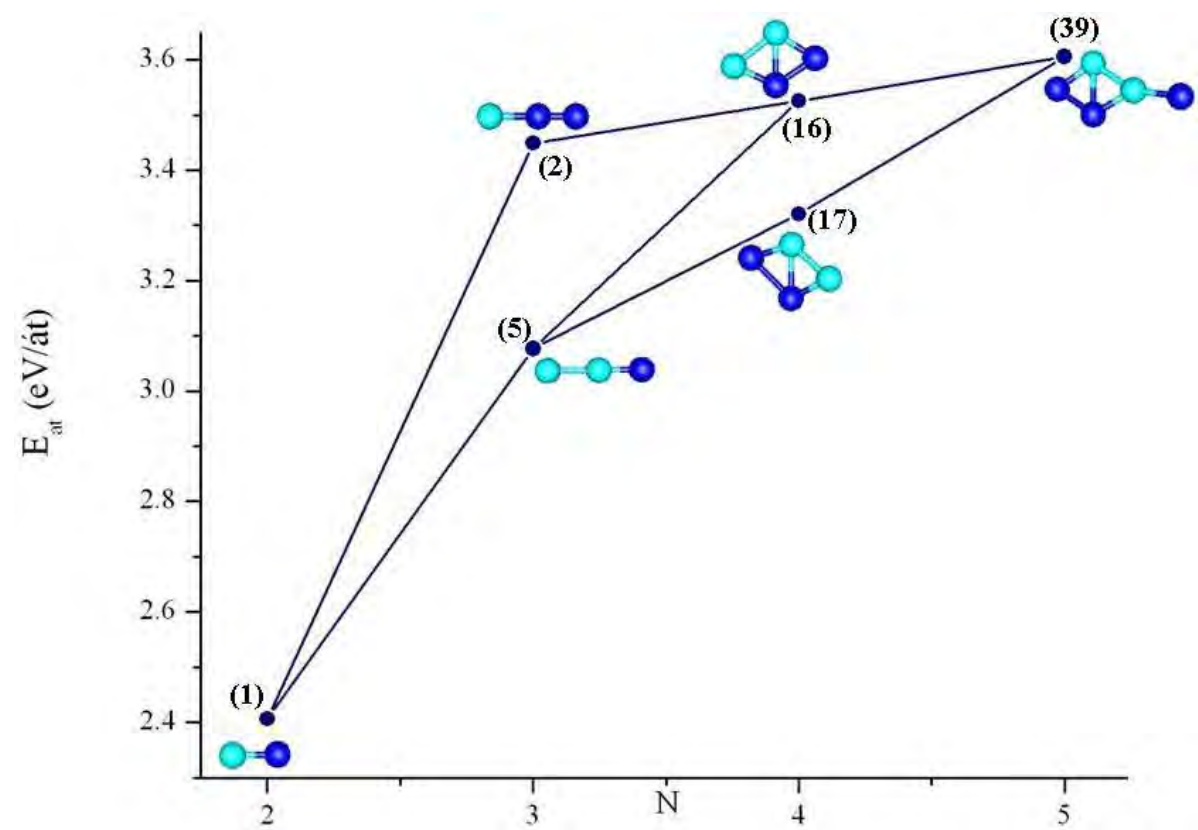

Figura 4.17.d Patrón de crecimiento de los trímeros (2) y (5); y tetrámeros (16) y (17).

El patrón de crecimiento del pentámero (39) se muestra en la Figura 4.17.d. Este pentámero puede ser obtenido por varios caminos, como se observa en el patrón. Si se obtuviera a partir de (17), un átomo de $\mathrm{N}$ debería unirse a $\mathrm{B} 2$ del tetrámero, que presenta carga ESP positiva de 0.07 ee (Tabla 4.8). Por otro lado, si este pentámero creciera de (16), el N3 debería atacar una zona de ESP positiva aún más pequeña, $0.01|\mathrm{e}|$ en B1.

La obtención del tetrámero (16) también posee dos posibilidades. Si crece por (5), un átomo de nitrógeno debe unirse a N1, de carga ESP negativa y a B1, de ESP positiva. Si creciera por el camino de (2) sería más favorable ya que un átomo de boro atacaría donde ambos átomos de los extremos de (2), B1 y N2, poseen carga ESP negativa, siguiendo con la tendencia mostrada anteriormente que los agregados donde átomos de boro se unen a zonas susceptibles de ataque electrofílico presentan mayor $\mathrm{E}_{\mathrm{at}}$.

Finalmente, los crecimientos más probables propuestos para cada trímero son:
$(1) \rightarrow(2) \rightarrow(12) \rightarrow(25)$
(Figura 4.17.a)
$(1) \rightarrow(3) \rightarrow(12) \rightarrow(25)$
(Figura 4.17.a)
$(1) \rightarrow(4) \rightarrow(6) \rightarrow(24)$
(Figura 4.17.b)
(1) $\rightarrow$ (5) $\rightarrow$ (14) $\rightarrow$ (23)
(Figura 4.16.c)

Donde el crecimiento más probable tanto para (2) como para (3) llega al mismo pentámero, por lo que llegarán al mismo hexámero.

Asimismo, el crecimiento de (4) es el más probable para todos los agregados, obteniéndose un pentámero alternado $\mathrm{B} / \mathrm{N}$ con átomos de boro en sus extremos, de geometría lineal y estado 
electrónico doblete, el confórmero (24), que también presenta zonas susceptibles de ataque electrofílico en los átomos de boro (Tabla 4.9) y susceptibles de ataque nucleofílico sobre los átomos de nitrógeno, mostrando electrones desapareados en los átomos de boro terminales (Figura 4.7).

\subsection{Conclusiones}

Se han encontrado las geometrías, energías de atomización, frecuencias de vibración armónicas, potenciales electrostáticos moleculares, cargas ESP, densidades de espín y patrones de crecimiento de clusters de $\mathrm{B}$ y N. En los trímeros y tetrámeros las energías de atomización encontradas son mayores que las reportadas en literatura, como se muestra en las Tablas 4.1 y 4.3, lo que indica que la metodología seguida para lograr estructuras estables es exitosa.

El dímero BN presenta estado electrónico triplete con una energía de atomización de 2.41 eV/át. El trímero más estable encontrado es el agregado BNB (4), de estado electrónico doblete, estructura lineal y una $\mathrm{E}_{\mathrm{at}}$ de $3.86 \mathrm{eV} /$ át. Asimismo, todos los agregados de tres átomos poseen estado electrónico doblete. El tetrámero NBNN (12) es el más estable, de estructura lineal, con estado electrónico singlete y una $\mathrm{E}_{\mathrm{at}}$ de $4.22 \mathrm{eV} / a ́$. El pentámero más estable es BNBNB (24), de estado electrónico doblete, con tres átomos de boro y dos de nitrógeno alternados en su estructura lineal.

En los agregados binarios $\mathrm{B} / \mathrm{N}$, encontramos que los movimientos de wagging, scissoring, rocking y twisting se encuentran entre $26-787 \mathrm{~cm}^{-1}$; los estiramientos simétricos y asimétricos B-N y B-B se encuentran entre 516 y $2023 \mathrm{~cm}^{-1}$; por último, los estiramientos simétricos y asimétricos N-N se encuentran a frecuencias altas, de $1905 \mathrm{~cm}^{-1}$ a $2330 \mathrm{~cm}^{-1}$. Se observan movimientos acoplados, especialmente en agregados que contienen ciclos en su estructura.

Los potenciales electrostáticos moleculares permiten explicar el crecimiento de los clusters. El crecimiento más probable es BN (1) $\rightarrow$ BNB (4) $\rightarrow$ BNBN (6) $\rightarrow$ BNBNB (24). El sistema $\mathrm{BN}$ tiende a crecer hacia agregados lineales, donde el átomo que se une actúa como electrófilo sobre un átomo de $\mathrm{B}$ o de $\mathrm{N}$. La densidad de espín no parece ser un condicionamiento del crecimiento, ya que los clusters no siguen una tendencia de en qué zona concentran electrones desapareados. 


\section{Estudio de la estructura, modos vibracionales y patrones de crecimiento de clusters de aluminio y fósforo}

Los agregados formados por aluminio y fósforo han sido estudiados últimamente por varios grupos, tanto de manera teórica como experimental ${ }^{81}$, con el objeto de conocer sus propiedades estructurales, vibracionales, electrónicas y ópticas. Estos estudios están motivados por el interés de conocer y entender el mecanismo de crecimiento de las películas delgadas de Al/P con el objeto de encontrar nanoestructuras semiconductoras novedosas que sean útiles tecnológicamente y explorar estructuras que sean isoelectrónicas con agregados de silicio, material ampliamente utilizado en dispositivos electrónicos. Asimismo, los trabajos experimentales se han centrado en encontrar una manera de sintetizar y conseguir estructuras estables de Al/P para su posterior estudio espectroscópico ${ }^{82}$.

Por otro lado, los trabajos teóricos encontrados en la literatura hasta el momento, se interesan en conocer las propiedades de agregados pequeños, proponiendo algunas estructuras. AlLaham y colaboradores ${ }^{83}$ determinaron las estructuras de más baja energía para agregados de tipo (AlP $)_{n}(n=1-4)$, usando Hartree-Fock y teoría de perturbaciones de segundo orden (MP2) con la base 6-31G*. Tomasulo y Ramakrishna ${ }^{82}$ exploraron agregados $(\mathrm{AlP})_{n}(n=1-$ 6), usando DFT/LDA y Car-Parinello con ondas planas y obtuvieron estructuras significativamente diferentes a clusters de $\mathrm{Si}_{2 n}$ isoelectrónicos. Feng y Balasubramanian ${ }^{84}$ realizaron cálculos químico-cuánticos a niveles CASSCF y MRSDCI con psuedopotenciales que retienen los electrones $3 \mathrm{~s}^{2} 3 \mathrm{p}^{1}$ y $3 \mathrm{~s}^{2} 3 \mathrm{p}^{3}$ del $\mathrm{Al}$ y $\mathrm{P}$, respectivamente; en clusters neutros y cargados de $\mathrm{Al}_{n} \mathrm{P}_{m}$ con cinco o menos átomos. Archibong y coautores ${ }^{85}$ calcularon estructuras electrónicas y energías para $\mathrm{AlP}_{2}, \mathrm{Al}_{2} \mathrm{P}_{2}, \mathrm{Al}_{3} \mathrm{P}, \mathrm{Al}_{3} \mathrm{P}_{3}$ y sus contrapartes iónicas usando $\mathrm{B} 3 \mathrm{LYP} / 6-311+\mathrm{G}(2 \mathrm{df})$ y CCSD $(\mathrm{T})^{7}$. Costales y colaboradores ${ }^{86}$ investigaron las estructuras y modos vibracionales de (AlP $)_{n}(n=1-3)$ utilizando el funcional de intercambio y correlación BPW91 y un conjunto de bases numéricas doble- $\zeta$, mientras que Qu y Bian ${ }^{87}$ realizaron cálculos similares hasta un tamaño de $n=4$, utilizando B3LYP y bases $6-31 \mathrm{G}(\mathrm{d})$ y 6 $311++\mathrm{G}(2 \mathrm{~d}, 2 \mathrm{p})$. Guo y colaboradores ${ }^{88}$ estudiaron las estructuras, estados electrónicos y estabilidades de agregados neutros y cargados de $\mathrm{Al}_{n} \mathrm{P}_{m}$ con $(m+n=3-6)$ a nivel B3LYP/6$311+\mathrm{G}(\mathrm{d})$ y con $(m+n=2-5)$ a nivel B3LYP/6-311+G(2df). Burril y Grein ${ }^{75}$ estudiaron las propiedades estructurales de clusters del tipo $\mathrm{X}_{2} \mathrm{Y}_{2}(\mathrm{X}=\mathrm{B}, \mathrm{Al}, \mathrm{Ga} ; \mathrm{Y}=\mathrm{N}, \mathrm{P}, \mathrm{As})$ usando DFT, el funcional B3LYP y el conjunto de funciones base 6-311+G(3df). En los trabajos de Karamanis ${ }^{89}$ estudiaron agregados de $(\mathrm{AlP})_{n}(n=2-9)$, con énfasis en sus momentos dipolares y hiperpolarizabilidades. Zhao y coautores ${ }^{81}$, consideraron clusters $\mathrm{Al}_{n} \mathrm{P}_{m}$ hasta un total de 18 átomos $(n+m=18)$ usando el funcional de intercambio y correlación BLYP y conjuntos de funciones base numéricas doble- $\zeta$ con funciones de polarización tipo $\mathrm{d}$.

En el presente capítulo informamos los resultados obtenidos para agregados binarios de $\mathrm{Al}_{n} \mathrm{P}_{m}$, con $n=1-4, m=1-4$ y $n+m \leq 5$. Se reportan propiedades estructurales, energías de atomización y frecuencias vibracionales para los agregados más estables.

El estudio de los patrones de crecimiento se realizó conformando los agregados más allá del dímero por la adición de átomos de $\mathrm{Al}$ o $\mathrm{P}$. El análisis de los patrones de crecimiento se llevó a cabo por medio de potenciales electrostáticos moleculares (MEP), densidades de espín y cargas derivadas de potenciales electrostáticos.

\subsection{Estructuras, energías y modos vibracionales}

Dímero AlP 
El estado fundamental del dímero AlP presenta estado electrónico triplete y una energía de atomización de $1.13 \mathrm{eV} /$ át (Tabla 5.1). La distancia de enlace de equilibrio Al-P es de $2.23 \AA$. La estructura de equilibrio se detalla en la Figura 5.1. Tomasuolo y Ramakrishna ${ }^{82}$ encontraron que el dímero posee una distancia de enlace de $2.05 \AA$. La distancia de enlace AlP experimental es de $2.40 \AA^{81}$.

\section{Trímeros}

Se obtuvieron cuatro agregados para $\mathrm{Al} / \mathrm{P}$, presentando estado electrónico doblete los agregados (3) y (4) y estado electrónico cuatriplete (2) y (5) (Tabla 5.1). El trímero de mayor $\mathrm{E}_{\mathrm{at}}$ es (3), de geometría no lineal y con dos átomos de fósforo y uno de aluminio es su estructura (Figura 5.1). El aumento de la energía de atomización se presenta de la siguiente manera: (2) $<$ (5) $<$ (4) $<$ (3). Los modos vibracionales del dímero y los trímeros se detallan en la Tabla 5.2.

Tabla 5.1. Multiplicidades de espín $\left(\mathrm{M}_{\mathrm{S}}\right)$, energías de atomización $\left(\mathrm{E}_{\mathrm{at}}\right)$ en eV/át, del dímero $\mathrm{y}$ trímeros. a se refiere a datos experimentales.

\begin{tabular}{c|c|c} 
Agregado & $\mathbf{M}_{\mathbf{s}}$ & $\mathbf{E}_{\mathbf{a t}}$ \\
\hline $\mathbf{( 1 )}$ & 3 & 1.13 \\
\hline & $3^{83,88}$ & \\
\hline & & $2.20^{81 \mathrm{a}}$ \\
\hline & & $2.11^{81}$ \\
\hline $\mathbf{( 2 )}$ & 4 & 1.02 \\
\hline $\mathbf{( 3 )}$ & 2 & 1.94 \\
\hline $\mathbf{( 4 )}$ & 2 & 1.78 \\
\hline & $2^{88}$ & \\
\hline $\mathbf{( 5 )}$ & 4 & 1.50 \\
\hline & $2^{85,88}$ &
\end{tabular}

Guo y coautores ${ }^{88}$ reportan que los agregados (4) y (5) presentan estado electrónico doblete, estando en desacuerdo con lo encontrado en este trabajo en el caso del agregado (5).

(1)

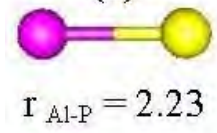

(4)

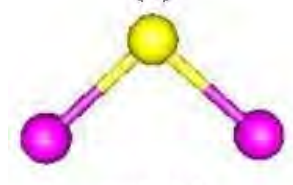

$\mathrm{r}_{\mathrm{A} 1 \mathrm{P}}=2.24$

$\mathrm{a}_{\text {Al-P-A. }}=95.7$
(2)

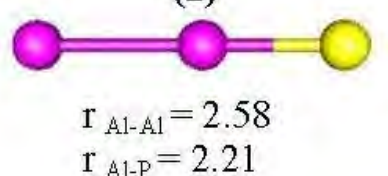

(5)

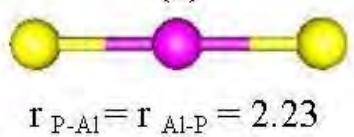

$\mathrm{r}_{\mathrm{P}-\mathrm{A} 1}=\mathrm{r}_{\mathrm{Al}-\mathrm{P}}=2.23$

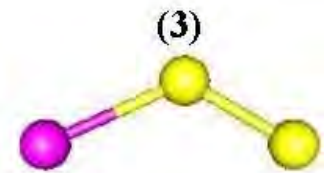

$\mathrm{r}_{\text {AI-P }}=2.39$

$\mathrm{r}_{\mathrm{P}-\mathrm{P}}=1.97$

$\mathrm{a}_{\text {A1-P-P }}=123.8$

Figura 5.1. Geometrías de equilibrio para el dímero de partida y los trímeros. Las distancias (r) están expresadas en $\AA$ y los ángulos (a) en ${ }^{\circ}$. Los ángulos diedros son $0.0^{\circ}$ y los ángulos planos que no se especifican 180.0, para todos los casos.

Por la Figura 5.1 se evidencia que, para los trímeros Al/P, la distancia $\mathrm{Al}-\mathrm{Al}$ es de $2.58 \AA$, la distancia P-P de $1.97 \AA$ y la distancia Al-P está entre 2.21 y $2.39 \AA$ A. Asimismo, se observa que la distancia del dímero es igual a la distancia Al-P del trímero simétrico (5). 
Zhao y coautores ${ }^{81}$ informan una distancia de enlace de $2.22 \AA$ para el dímero y citan en su trabajo, una distancia experimental de $2.40 \AA$. Por otro lado, Tomasuolo y Ramakrishna ${ }^{82}$ encontraron que el dímero posee una distancia de enlace de $2.05 \AA$. Al-Laham y colaboradores $^{83}$ reportan una distancia de enlace Al-P del dímero de $2.43 \AA$ A. Archibong y coautores $^{85}$ reportaron distancias de enlace Al-P y P-P de $2.61 \AA$ y $1.97 \AA$, respectivamente, con un ángulo P-P-Al de $44.4^{\circ}$, para (3), al igual que Guo y colaboradores ${ }^{88}$, quienes también reportan una distancia de enlace de 2.14 Å para el dímero; para (4) informan distancia Al-P de $2.24 \AA$ y un ángulo Al-P-Al de $91.2^{\circ}$. Se observa, por la Figura 5.1, que la mayor distancia Al-P de los agregados de hasta tres átomos es la que se presenta en (3), adyacente a una unión P-P.

Tabla 5.2. Modos normales de vibración $\left(\omega_{\mathrm{e}}\right)$ en $\mathrm{cm}^{-1}$, del dímero y los trímeros. a se refiere a datos experimentales.

\begin{tabular}{c|c} 
Agregado & $\boldsymbol{\omega}_{\mathbf{e}}$ \\
\hline $\mathbf{( 1 )}$ & 455 \\
\hline & $379^{81 \mathrm{a}}$ \\
\hline & $372^{81}$ \\
\hline & $381^{83}$ \\
\hline & $457^{88}$ \\
\hline $\mathbf{( 2 )}$ & $56,78,230,520$ \\
\hline $\mathbf{( 3 )}$ & $73,330,657$ \\
\hline $\mathbf{( 4 )}$ & $70,228,431$ \\
\hline & $68,241,435^{88}$ \\
\hline $\mathbf{( 5 )}$ & $98,108,337,496$ \\
\hline & $157,286,693^{85}$ \\
\hline & $155,285,692^{88}$
\end{tabular}

El estiramiento Al-P del dímero se encuentra a $455 \mathrm{~cm}^{-1}$. Respecto a los modos vibracionales de los trímeros, en frecuencias bajas presentan movimientos de tipo wagging y scissoring; en frecuencias intermedias estiramientos simétricos y asimétricos Al-P y Al-Al y a frecuencias mayores a $657 \mathrm{~cm}^{-1}$ estiramientos P-P. Los modos vibracionales encontrados para los agregados (1) y (4) están en buen acuerdo con cálculos realizados por Guo y colaboradores ${ }^{88}$. Por otro lado, el modo vibracional encontrado de manera experimental y reportado en la referencia 81 , difiere considerablemente a lo hallado en este trabajo.

\section{Tetrámeros}

Se calcularon los clusters binarios de cuatro átomos partiendo de los trímeros obtenidos. Asimismo, se consideró un agregado romboidal alternado, el agregado (13), con el objetivo de comparar con datos obtenidos por otros autores. Se obtuvieron ocho agregados estables, siete planos y uno no plano, (13), de los cuales el que presenta mayor $E_{a t}$ es (8), de $2.47 \mathrm{eV} /$ át, que posee estado electrónico singlete y geometría cíclica (Tabla 5.3). Los parámetros geométricos se muestran en la Figura 5.2 y los modos vibracionales en la Tabla 5.4. 
Tabla 5.3. Multiplicidades de espín $\left(\mathrm{M}_{\mathrm{S}}\right)$ y energías de atomización $\left(\mathrm{E}_{\mathrm{at}}\right)$ en eV/át, de los tetrámeros.

\begin{tabular}{c|c|c} 
Agregado & $\mathbf{M}_{\mathbf{s}}$ & $\mathbf{E}_{\mathbf{a t}}$ \\
\hline $\mathbf{( 6 )}$ & 3 & 2.09 \\
\hline $\mathbf{( 7 )}$ & 3 & 1.52 \\
\hline $\mathbf{( 8 )}$ & 1 & 2.47 \\
\hline & $1^{88}$ & \\
\hline $\mathbf{( 9 )}$ & 3 & 2.15 \\
\hline $\mathbf{( 1 0 )}$ & 3 & 1.90 \\
\hline & $3^{87}$ & $2.71^{87}$ \\
\hline $\mathbf{( 1 1 )}$ & 1 & 1.80 \\
\hline $\mathbf{( 1 2 )}$ & 1 & 2.04 \\
\hline & $1^{85,88}$ & \\
\hline $\mathbf{( 1 3 )}$ & 3 & 2.14 \\
\hline & $1^{71,81,83,85,86,88}$ & $2.39^{81}$ \\
\hline & $1^{87}$ & $3.09^{87}$ \\
\hline
\end{tabular}

Analizando la Tabla 5.2, se observa que el aumento en la energía de atomización es: $(7)<$ (11) $<$ (10) $<$ (12) $<$ (6) $<$ (13) $<$ (9) $<$ (8). De un total de ocho tetrámeros, cinco poseen dos electrones desapareados. Otros autores encontraron al agregado (13) como más estable, lo reportan como totalmente simétrico, plano y de estado electrónico singlete ${ }^{71,83,86,87,88}$. Cabe destacar que los estudios correspondientes a las referencias $71,83,86$ y 88 fueron realizados sobre el agregado de capa cerrada, únicamente. También es importante mencionar que Tomasulo y Ramakrishna ${ }^{82}$ estudiaron el agregado romboidal (13) de capa cerrada, pero no informan datos que se puedan comparar con los obtenidos en este trabajo. Asimismo, es relevante resaltar que en el trabajo de Zhao y colaboradores ${ }^{81}$ no está claro en qué formato se encuentran las energías informadas, habiendo discrepancias en las unidades reportadas en las tablas del mismo. 
(6)
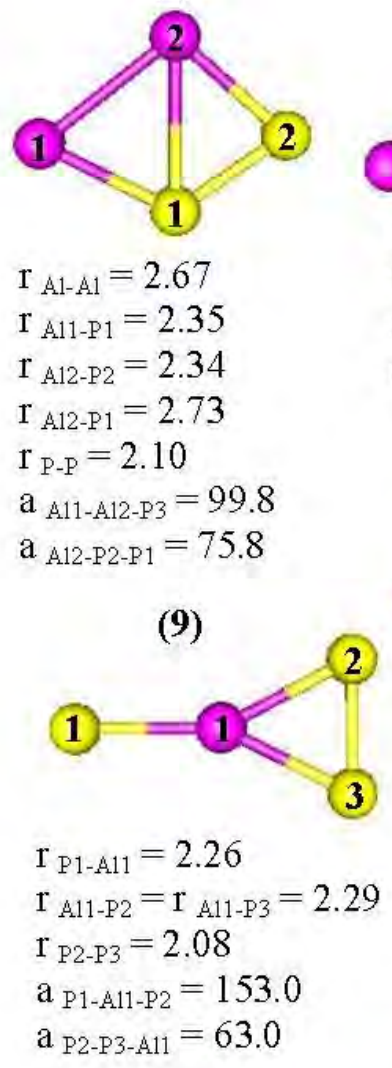

(12)

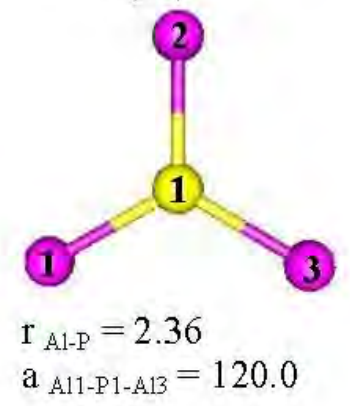

(7)

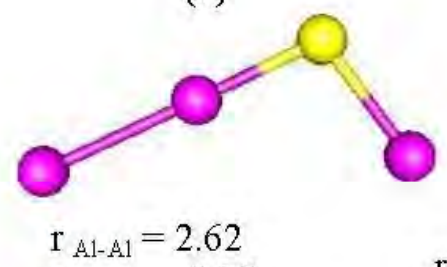

$\mathrm{r}_{\mathrm{A} 12-\mathrm{P} 1}=2.18$

$\mathrm{r}_{\mathrm{P} 1-\mathrm{A} 13}=2.28$

$\mathrm{a}_{\mathrm{A} 12-\mathrm{P} 1-\mathrm{A} 13}=99.9$

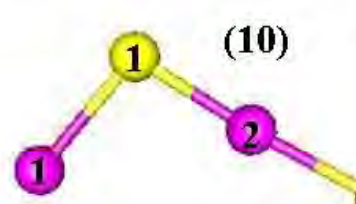

$r_{\text {A11-P1 }}=2.33$

$\mathrm{r}_{\mathrm{P} 1-\mathrm{A} 12}=2.16$

$r_{\mathrm{A} 12-\mathrm{P} 2}=2.26$

$\mathrm{a}_{\mathrm{A} 11-\mathrm{P} 1-\mathrm{P} 2}=97.9$

(13)

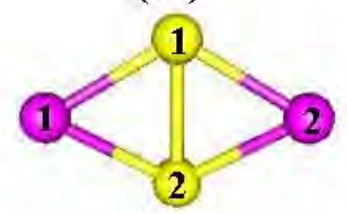

$\mathrm{r}_{\mathrm{A} 11-\mathrm{P} 1}=\mathrm{r}_{\mathrm{A} 11-\mathrm{P} 3}=2.67$

$\mathrm{r}_{\mathrm{P}-\mathrm{P}}=2.10$

$\mathrm{r}_{\mathrm{A} 11-\mathrm{P} 2}=2.46$

$\mathrm{a}_{\mathrm{P} 1-\mathrm{A} 11-\mathrm{P} 3}=104.14$

$\mathrm{a}_{\mathrm{A} 11-\mathrm{P} 3-\mathrm{P} 2}=67.9$

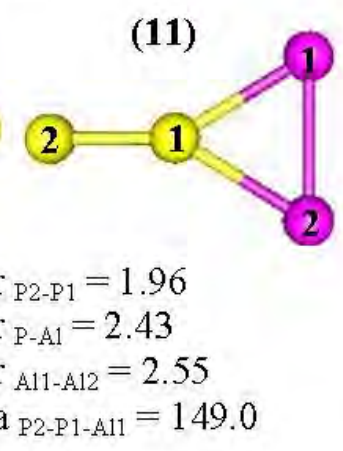

\section{2}

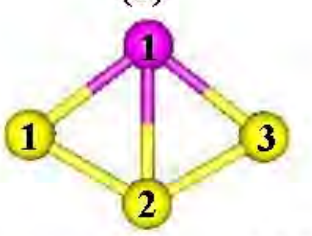

$\mathrm{r}_{\mathrm{P}-\mathrm{A} .1}=2.37$

$\mathrm{r}_{\mathrm{P} 1-\mathrm{P} 2}=2.34$

$\mathrm{a}_{\mathrm{A} 11-\mathrm{P} 1-\mathrm{A} 12}=118.0$

$\mathrm{a}_{\mathrm{P} 1-\mathrm{A} 12-\mathrm{P} 2}=59.2$

$\mathrm{d}_{\mathrm{P}-\mathrm{A} 11-\mathrm{P} 1-\mathrm{A} 12}=19.2$ 
(12) y reportan una distancia de enlace Al-P de $2.35 \AA$, en muy buen acuerdo con lo encontrado en este trabajo. Asimismo, reportan al rombo plano de estado electrónico singlete como más estable, con uniones Al-P de $2.53 \AA$ y P-P de $2.08 \AA$ con un ángulo P-Al-P de $48.2^{\circ}$. Costales y coautores ${ }^{87}$ reportan, para el agregado (13) una estructura plana, de capa cerrada y con distancias de enlace Al-P y P-P de 2.53 y 2.08 , respectivamente. Guo y colaboradores $^{88}$ encontraron para una estructura similar a (8) una unión Al1-P1 de $2.29 \AA$, una distancia de enlace Al1-P2 de $2.45 \AA$ y una distancia de enlace P2-P1 de $2.09 \AA$, coincidiendo sólo en esta última con este trabajo. Asimismo, estudiaron un agregado similar a (12), con una distancia de enlace Al-P de $2.35 \AA$ y un ángulo Al-P-Al de $119.9^{\circ}$ y un agregado similar a (13), el cual reportan como plano y con estado electrónico singlete, con una distancia P-P de $2.07 \AA$ y un enlace Al-P de $2.53 \AA$.

Tabla 5.4. Frecuencias vibracionales $\left(\omega_{\mathrm{e}}\right)$ de los tetrámeros, en $\mathrm{cm}^{-1}$. * indica un modo doblemente degenerado.

\begin{tabular}{c|c} 
Agregado & $\boldsymbol{\omega}_{\mathbf{e}}$ \\
\hline $\mathbf{( 6 )}$ & $111,202,225,336,371,519$ \\
\hline $\mathbf{( 7 )}$ & $42,81,97,179,320,504$ \\
\hline $\mathbf{( 8 )}$ & $195,287,353,365,501,568$ \\
\hline & $196,292,359,367,504,571^{134}$ \\
\hline $\mathbf{( 9 )}$ & $80,114,295,368,457,619$ \\
\hline $\mathbf{( 1 0 )}$ & $59,107,133,327,366,561$ \\
\hline & $57,104,118,348,420,583^{133}$ \\
\hline $\mathbf{( 1 1 )}$ & $82,104,222,257,315,658$ \\
\hline $\mathbf{( 1 2 )}$ & $20,69,70,297,415^{*}$ \\
\hline & $20,70,297,415^{85}$ \\
\hline & $20,70,297,415^{88}$ \\
\hline $\mathbf{( 1 3 )}$ & $56,236,310,312,328,437$ \\
\hline & $96,149,239,280,353,644^{83}$ \\
\hline & $88,159,349,274,276,588^{85}$ \\
\hline & $92,164,277,289,341,572^{86}$ \\
\hline & $94,159,272,276,342,580^{87}$ \\
\hline & $87,159,275,277,341,598^{88}$
\end{tabular}

En los tetrámeros se presentan movimientos de tipo wagging y scissoring en frecuencias bajas; en frecuencias intermedias se presentan estiramientos simétricos y asimétricos $\mathrm{Al}-\mathrm{Al}$ y Al-P y a más de $568 \mathrm{~cm}^{-1}$, estiramientos P-P. Los resultados de modos vibracionales encontrados para (8), (10) y (12) se encuentran en buen acuerdo con los informados en las referencias. Sin embargo, los resultados de los modos vibracionales reportados en las referencias para el agregado (13) no coinciden con los encontrados en este trabajo, debido a que los otros trabajos se centran en estudiar el rombo alternado de estado electrónico singlete, el cual posee parámetros geométricos muy distintos a (13), Figura 5.3.

Geometría de rombo alternado singlete y análisis de átomos en moléculas

Con el objetivo de conocer las características de los enlaces en el agregado (13) y el mismo agregado, de estado electrónico singlete, se realizó un análisis de átomos en moléculas (AIM). Para ello, se optimizó previamente la estructura del rombo singlete, (13s), cuyas características estructurales se muestran en la Figura 5.3. 


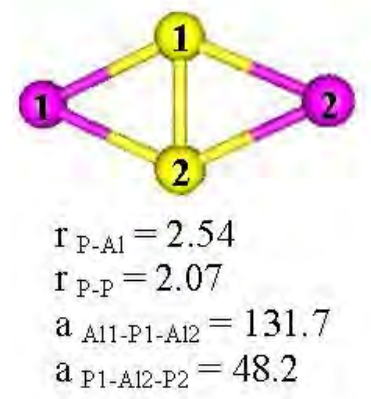

Figura 5.3. Estructura optimizada del rombo alternado, agregado (13s), estado electrónico singlete.

Se observa en la figura que la geometría encontrada para el rombo alternado estado electrónico singlete coincide ampliamente con la geometría reportada por otros autores. Asimismo, la energía de atomización encontrada para este agregado (2.29 eV/át), supera el valor reportado por Zhao y colaboradores ${ }^{81}$, aunque es menor que lo reportado por $\mathrm{Qu} \mathrm{y}$ $\operatorname{Bian}^{87}$.

Tabla 5.5. Propiedades de los puntos críticos encontrados para los distintos enlaces del agregado (13) en estados electrónicos singlete (13s) y triplete (13), la densidad electrónica $\rho(r)$, el laplaciano de la densidad $\nabla^{2} \rho(r)$ y la elipticidad $\varepsilon$, todos en u.a.

\begin{tabular}{c|c|c|c} 
Punto Crítico & Parámetro & $\mathbf{( 1 3 s )}$ & $\mathbf{( 1 3 )}$ \\
\hline P1-Al1 & $\rho(r)$ & 0.04 & 0.05 \\
& $\nabla^{2} \rho(r)$ & 0.05 & 0.08 \\
& $\varepsilon$ & 1.34 & 0.06 \\
\hline P1-A12 & $\rho(r)$ & 0.04 & 0.05 \\
& $\nabla^{2} \rho(r)$ & 0.05 & 0.08 \\
& $\varepsilon$ & 1.34 & 0.06 \\
\hline P2-A11 & $\rho(r)$ & 0.04 & 0.05 \\
& $\nabla^{2} \rho(r)$ & 0.05 & 0.08 \\
& $\varepsilon$ & 1.34 & 0.06 \\
\hline P2-A12 & $\rho(r)$ & 0.04 & 0.05 \\
& $\nabla^{2} \rho(r)$ & 0.05 & 0.08 \\
& $\varepsilon$ & 1.34 & 0.06
\end{tabular}

Se puede apreciar por la Tabla 5.5 que para ambos agregados se encontraron los mismos puntos críticos del enlace y que éstos son totalmente simétricos (Tabla 5.4, Figuras 5.2 y 5.3). Sin embargo, el valor de elipticidad encontrado para el estado electrónico singlete (13s) es mayor a uno, lo cual demuestra que los enlaces de (13s) son inestables y la estructura tenderá a relajarse a otra estructura más estable y posiblemente de mayor multiplicidad, como ser el estado electrónico triplete, (13).

\section{Pentámeros}

A partir de los tetrámeros antes descriptos se generaron los pentámeros, de los cuales 22 resultaron estables. En la Tabla 5.6 se listan las multiplicidades de espín y energías de atomización correspondientes. Las geometrías de equilibrio encontradas se presentan en las Figuras 5.4 a 5.6. 
Tabla 5.6. Multiplicidades de espín $\left(\mathrm{M}_{\mathrm{S}}\right)$ y energías de atomización $\left(\mathrm{E}_{\mathrm{at}}\right)$ en eV/át, de los pentámeros.

\begin{tabular}{c|c|c} 
Agregado & $\mathbf{M}_{\mathbf{s}}$ & $\mathbf{E}_{\mathbf{a t}}$ \\
\hline $\mathbf{( 1 4 )}$ & 6 & 1.64 \\
\hline $\mathbf{( 1 5 )}$ & 2 & 2.17 \\
\hline $\mathbf{( 1 6 )}$ & 6 & 1.77 \\
\hline $\mathbf{( 1 7 )}$ & 2 & 2.46 \\
\hline & $2^{88}$ & \\
\hline $\mathbf{( 1 8 )}$ & 4 & 2.26 \\
\hline $\mathbf{( 1 9 )}$ & 4 & 2.16 \\
\hline $\mathbf{( 2 0 )}$ & 4 & 2.20 \\
\hline $\mathbf{( 2 1 )}$ & 4 & 2.29 \\
\hline $\mathbf{( 2 2 )}$ & 2 & 2.31 \\
\hline $\mathbf{( 2 3 )}$ & 2 & 2.47 \\
\hline $\mathbf{( 2 4 )}$ & 2 & 2.19 \\
\hline $\mathbf{( 2 5 )}$ & 2 & 2.36 \\
\hline $\mathbf{( 2 6 )}$ & 4 & 1.77 \\
\hline $\mathbf{( 2 7 )}$ & 2 & 2.21 \\
\hline $\mathbf{( 2 8 )}$ & 4 & 1.74 \\
\hline & $2^{88}$ & \\
\hline $\mathbf{( 2 9 )}$ & 2 & 2.30 \\
\hline $\mathbf{( 3 0 )}$ & 6 & 1.65 \\
\hline $\mathbf{( 3 1 )}$ & 2 & 2.47 \\
\hline $\mathbf{( 3 2 )}$ & 4 & 1.94 \\
\hline $\mathbf{( 3 3 )}$ & 2 & 2.20 \\
\hline $\mathbf{( 3 4 )}$ & 2 & 2.13 \\
\hline $\mathbf{( 3 5 )}$ & 4 & 2.14
\end{tabular}

Se observa en la Tabla 5.6, que el pentámero más estable es (23), agregado no plano, con una $\mathrm{E}_{\text {at }}$ de $2.473 \mathrm{eV} / a$ á (expresado con tres decimales) y estado electrónico doblete con 4 átomos de fósforo y uno de aluminio (Figura 5.5), muy cercano a (31), con $2.471 \mathrm{eV} /$ át, con dos átomos de aluminio y tres de fósforo (Figura 5.6). Asimismo, se ve que aquellos agregados con 5 electrones desapareados ((14), (16) y (30)), son los menos estables de la serie. La mayoría de los agregados de cinco átomos resultaron no planos. Se observa similitud entre los agregados (26) y (28), por un lado, y entre los agregados (25) y (29), por otro. La energía de atomización aumenta de la siguiente manera: (14) $<$ (30) $<$ (28) $<$ (26) $<$ (16) $<$ (32) $<$ (34) $<$ (35) $<$ (19) $<$ (15) $<$ (24) $<$ (33) $<$ (20) $<$ (27) $<$ (18) $<$ (21) $<$ (29) $<$ (22) $<$ (25) $<$ (17) $<$ (31) $<$ (23), siendo las energías de (16) y (26) 1.775 y 1.770 , las de (32) y (19), 2.197 y 2.205 , las de (23) y (31), 2.473 y 2.471 , respectivamente. 

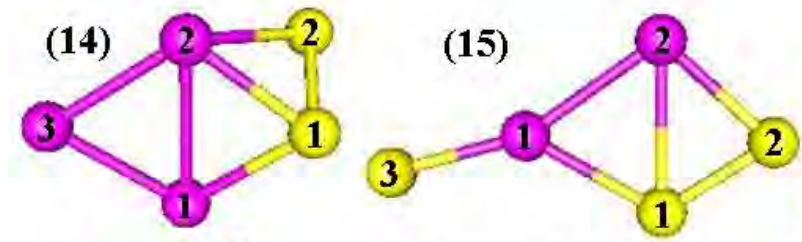

$$
\begin{aligned}
& \mathrm{r}_{\mathrm{A} 13-\mathrm{A} 12}=2.60 \\
& \mathrm{r}_{\mathrm{A} 13-\mathrm{A} 11}=2.54 \\
& \mathrm{r}_{\mathrm{A} 12-\mathrm{P} 1}=2.39 \\
& \mathrm{r}_{\mathrm{A12}-\mathrm{P} 2}=2.67 \\
& \mathrm{r}_{\mathrm{A} 12-\mathrm{A} 11}=2.87 \\
& \mathrm{r}_{\mathrm{A} 11-\mathrm{P} 1}=2.47 \\
& \mathrm{r}_{\mathrm{P} 2 \mathrm{P} 1}=2.14 \\
& \mathrm{a}_{\mathrm{A} 13-\mathrm{A} 12-\mathrm{P} 1}=109.6 \\
& \mathrm{a}_{\mathrm{A} 12-\mathrm{P} 1-\mathrm{P} 2}=71.9 \\
& \mathrm{~d}_{\mathrm{A} 11-\mathrm{P} 1-\mathrm{P} 2-\mathrm{A} 12}=68.8
\end{aligned}
$$

$$
\begin{aligned}
& \mathrm{r}_{\mathrm{P} 3-\mathrm{A} 11}=2.20 \\
& \mathrm{r}_{\mathrm{A} 11-\mathrm{A} 12}=2.57 \\
& \mathrm{r}_{\mathrm{A} 12-\mathrm{P} 2}=2.35 \\
& \mathrm{r}_{\mathrm{P} 1 \mathrm{-P} 2}=2.04 \\
& \mathrm{r}_{\mathrm{A} 12-\mathrm{P} 1}=2.70 \\
& \mathrm{r}_{\mathrm{A} 11-\mathrm{P} 1}=2.44 \\
& \mathrm{a}_{\mathrm{P} 3-\mathrm{A} 11-\mathrm{A} 12}=159.9 \\
& \mathrm{a}_{\mathrm{A} 12-\mathrm{P} 2-\mathrm{P} 1}=75.4
\end{aligned}
$$

(16)
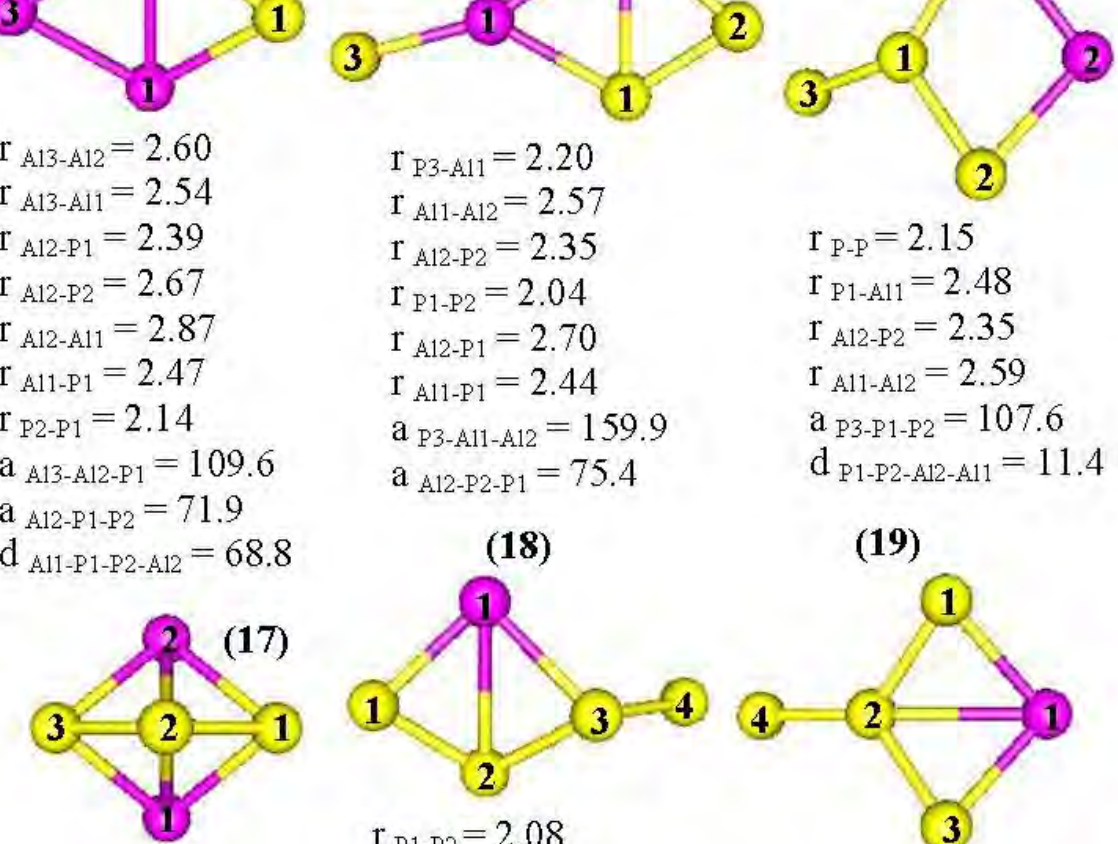

$$
\begin{aligned}
& \mathrm{r}_{\mathrm{P}-\mathrm{P}}=2.18 \\
& \mathrm{r}_{\mathrm{P}-\mathrm{A} 1}=2.48 \\
& \mathrm{a}_{\mathrm{P} 3-\mathrm{P} 2-\mathrm{P} 1}=102.7 \\
& \mathrm{a}_{\text {A11-P3-A12 }}=73.9 \\
& \mathrm{~d}_{\text {A12-P1-P2-P3 }}=39.4
\end{aligned}
$$

$$
\begin{aligned}
& r_{P 1-P 2}=2.08 \\
& r_{P 2-P 3}=2.22 \\
& r_{P 3-P 4}=2.16 \\
& r_{\text {A11-P1 }}=2.34 \\
& r_{\text {A11-P2 }}=.74 \\
& r_{\text {A11-P3 }}=2.41 \\
& a_{\text {P1-A11-P3 }}=92.9 \\
& d_{\text {P1-A11-P3-P4 }}=121.9
\end{aligned}
$$

$$
\begin{aligned}
& \mathrm{r}_{\mathrm{P}-\mathrm{P}}=2.15 \\
& \mathrm{r}_{\mathrm{P} 1-\mathrm{A} 11}=2.48 \\
& \mathrm{r}_{\mathrm{A} 12-\mathrm{P} 2}=2.35 \\
& \mathrm{r}_{\mathrm{A} 11-\mathrm{A} 12}=2.59 \\
& \mathrm{a}_{\mathrm{P} 3-\mathrm{P} 1-\mathrm{P} 2}=107.6 \\
& \mathrm{~d}_{\mathrm{P} 1-\mathrm{P} 2-\mathrm{A} 12-\mathrm{A} 11}=11.4
\end{aligned}
$$

(19)

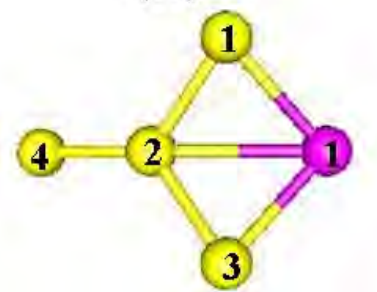

$$
\begin{aligned}
& r_{\mathrm{P} 1-\mathrm{P} 2}=\mathrm{r}_{\mathrm{P} 2-\mathrm{P} 3}=2.14 \\
& \mathrm{r}_{\mathrm{P}-\mathrm{P} 4}=2.04 \\
& \mathrm{r}_{\mathrm{A} 11-\mathrm{P} 1}=\mathrm{r}_{\mathrm{A11-P3}}=2.37 \\
& \mathrm{r}_{\mathrm{A} 11-\mathrm{P} 2}=2.71 \\
& \mathrm{a}_{\mathrm{P} 1-\mathrm{A} 11-\mathrm{P} 3}=92.1 \\
& \mathrm{a}_{\mathrm{P} 4-\mathrm{P} 2-\mathrm{P} 3}=127.1 \\
& \mathrm{~d}_{\text {A11-P1-P-P3 }}=29.7
\end{aligned}
$$

Figura 5.4. Geometrías de equilibrio de los pentámeros (14) a (19). Las distancias (r) están expresadas en Å y los ángulos planos (a) y diedros (d), en ${ }^{\circ}$. 


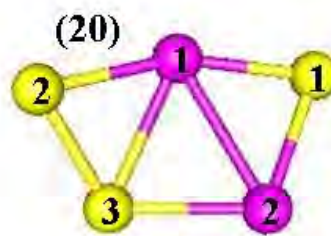

$\mathrm{r}_{\mathrm{P}-\mathrm{P}}=2.15$

$\mathrm{r}_{\mathrm{P} 2 \mathrm{~A}-\mathrm{A} 11}=2.25$

$\mathrm{r}_{\mathrm{A} 11-\mathrm{P} 1}=2.22$

$\mathrm{r}_{\mathrm{P} 1-\mathrm{A} 12}=2.32$

$\mathrm{r}_{\mathrm{A} 11-\mathrm{P} 3}=2.57$

$\mathrm{r}_{\mathrm{A} 12-\mathrm{P} 3}=2.45$

$\mathrm{r}_{\mathrm{A} 12-\mathrm{A} 11}=2.75$

$\mathrm{a}_{\mathrm{P} 2-\mathrm{A} 11-\mathrm{P} 1}=158.0$

$\mathrm{d}_{\mathrm{P} 2-\mathrm{A} 11-\mathrm{P} 1-\mathrm{A} 12}=11.9$

(23)

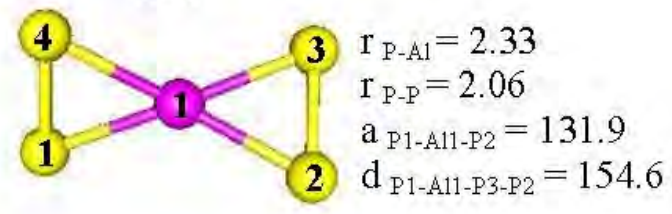

(21)

$\mathrm{r}_{\mathrm{P}-\mathrm{P}}=2.14$

$\mathrm{r}_{\mathrm{A} 11-\mathrm{P} 1}=2.33$

$\mathrm{r}_{\mathrm{A} 11-\mathrm{P} 3}=2.71$

$\mathrm{a}_{\mathrm{P2}-\mathrm{A} \text { A11-P4 }}=91.5$

$\mathrm{a}_{\mathrm{P} 2-\mathrm{A} 11-\mathrm{P} 1}=132.5$

$\mathrm{d}_{\mathrm{P} 1-\mathrm{Al1}-\mathrm{P} 4-\mathrm{P} 3}=130.0$

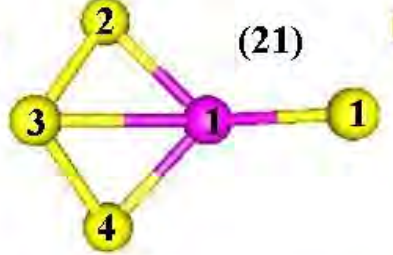

$\mathrm{r}_{\mathrm{P} 2-\mathrm{A} 11}=\mathrm{r}_{\text {P4-A11 }}=2.32$
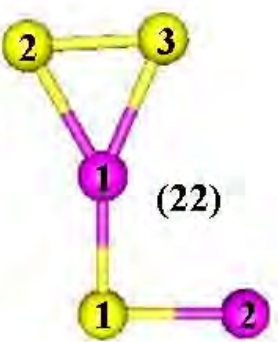

$\mathrm{r}_{\mathrm{P} 3-\mathrm{A} 11}=\mathrm{r}_{\mathrm{A} 11-\mathrm{P} 2}=\mathrm{r}_{\mathrm{P} 1-\mathrm{A} 12}=2.30$

$\mathrm{r}_{\mathrm{A} 11-\mathrm{P} 1}=2.21$

$\mathrm{r}_{\mathrm{P}-\mathrm{P}}=2.07$

$\mathrm{a}_{\mathrm{A} 11-\mathrm{P} 2-\mathrm{P} 3}=63.7$

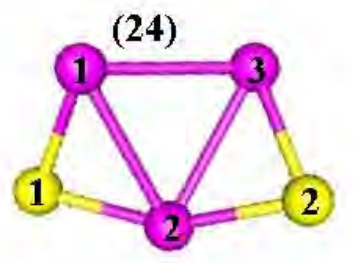

$$
\begin{aligned}
& \mathrm{r}_{\mathrm{P} 1-\mathrm{A} 11}=\mathrm{r}_{\mathrm{P} 2-\mathrm{A} 13}=2.28 \\
& \mathrm{r}_{\mathrm{P} 1-\mathrm{A} 12}=\mathrm{r}_{\mathrm{P} 2-\mathrm{A} 12}=2.21 \\
& \mathrm{r}_{\mathrm{A} 11-\mathrm{A} 13}=2.78 \\
& \mathrm{r}_{\mathrm{A} 11-\mathrm{A} 12}=\mathrm{r}_{\mathrm{A} 12-\mathrm{A} 13}=2.84 \\
& \mathrm{a}_{\mathrm{P} 1-\mathrm{A} 12-\mathrm{P} 2}=154.7 \\
& \mathrm{~d}_{\mathrm{A} 12-\mathrm{P} 2-\mathrm{A} 13-\mathrm{P} 1}=30.0
\end{aligned}
$$

Figura 5.5. Geometrías de equilibrio de los pentámeros (20) a (24). Las distancias (r) están expresadas en $\AA$ y los ángulos planos (a) y diedros (d), en ${ }^{\circ}$. 
(25)

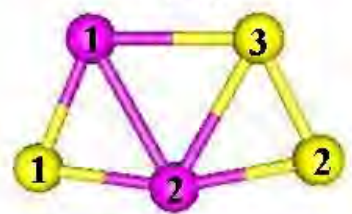

$\mathrm{r}_{\mathrm{P}-\mathrm{P}}=2.06$
$\mathrm{r}_{\mathrm{P} 1 \mathrm{~A} 11}=2.26$
$\mathrm{r}_{\mathrm{P} 1-\mathrm{A} 12}=2.22$
$\mathrm{r}_{\mathrm{A} 11-\mathrm{A} 12}=\mathrm{r}_{\mathrm{A} 12-\mathrm{P} 3}=2.67$
$\mathrm{r}_{\mathrm{A} 11-\mathrm{P} 3}=2.63$
$\mathrm{r}_{\mathrm{A} 12-\mathrm{P} 2}=2.30$
$\mathrm{a}_{\mathrm{P} 1-\mathrm{A} 12-\mathrm{P} 2}=161.4$

(29)

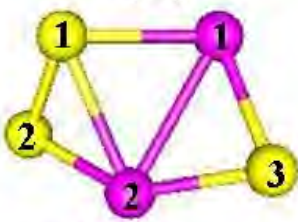

$\mathrm{r}_{\mathrm{P}-\mathrm{P}}=2.11$

$\mathrm{r}_{\mathrm{P} 2-\mathrm{A} 12}=2.27$

$\mathrm{r}_{\mathrm{A} 12-\mathrm{P} 3}=2.22$

$r_{P 3-A 11}=2.35$

$\mathrm{r}_{\mathrm{Al1-P1}}=2.45$

$\mathrm{r}_{\mathrm{A} 12 \mathrm{P} 1}=2.63$

$\mathrm{r}_{\mathrm{A} 11-\mathrm{A} 2}=2.82$

$\mathrm{a}_{\mathrm{P} 2-\mathrm{A} 12-\mathrm{P} 3}=141.6$

$\mathrm{d}_{\mathrm{P} 2-\mathrm{A} 1 \mathrm{-P} \text {-A.11 }}=14.6$

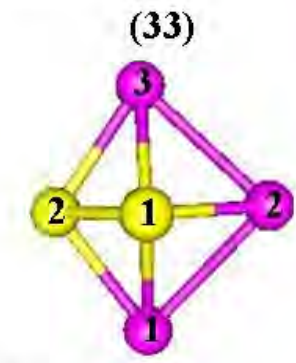

$\mathrm{r}_{\mathrm{P}-\mathrm{P}}=2.16$

$\mathrm{r}_{\mathrm{A} 12-\mathrm{P} 1}=2.41$

$\mathrm{r}_{\mathrm{A} 13-\mathrm{P} 2}=\mathrm{r}_{\mathrm{A} 11-\mathrm{P} 2}=2.42$

$\mathrm{r}_{\mathrm{A} 13-\mathrm{P} 1}=\mathrm{r}_{\mathrm{A} 11-\mathrm{P} 1}=2.68$

$r_{\text {Al-A1 }}=2.81$

$a_{\text {A13-P2-A.11 }}=103.3$

$\mathrm{d}_{\mathrm{A} 12-\mathrm{P} 1-\mathrm{P} 2-\mathrm{A} 11}=58.8$
(26)

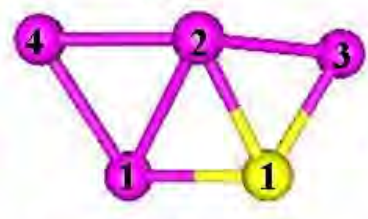

$\mathrm{r}_{\mathrm{P} 1-\mathrm{A} 11}=2.32$

$r_{\mathrm{P} 1-\mathrm{A} 13}=2.37$

$r_{P 1-A 12}=2.43$

$\mathrm{r}_{\mathrm{A} 11-\mathrm{A} .14}=2.63$

$\mathrm{r}_{\mathrm{A} 11-\mathrm{A} 12}=2.83$

$\mathrm{r}_{\mathrm{A} 12-\mathrm{A} 14}=2.55$

$\mathrm{r}_{\mathrm{A} 12-\mathrm{A} 13}=2.70$

$\mathrm{a}_{\mathrm{A} 14-\mathrm{A} 11-\mathrm{P} 1}=110.8$

$\mathrm{d}_{\mathrm{P} 1-\mathrm{A} 11-\mathrm{A} 14-\mathrm{A} 12}=58.3$

\section{(30)}

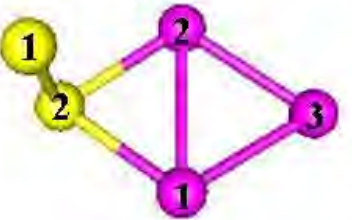

$\mathrm{r}_{\mathrm{P}-\mathrm{P}}=2.16$

$\mathrm{r}_{\mathrm{A} 11-\mathrm{A}_{113}}=\mathrm{r}_{\mathrm{A} 12-\mathrm{A} 13}=2.56$

$\mathrm{r}_{\mathrm{Al}-\mathrm{P}}=2.39$

$\mathrm{r}_{\mathrm{A} 11-\mathrm{A} 12}=2.81$

$\mathrm{a}_{\mathrm{A} 12-\mathrm{P} 2-\mathrm{P} 1}=96.0$

$\mathrm{d}_{\mathrm{P} 1-\mathrm{P} 2-\mathrm{A} 12-\mathrm{A} 13}=76.7$

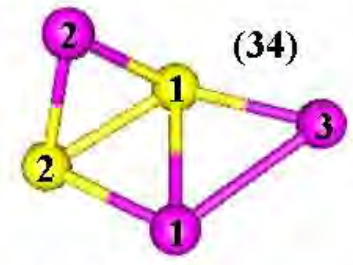

$\mathrm{r}_{\mathrm{P}-\mathrm{P}}=2.45$

$\mathrm{r}_{\mathrm{A} 12-\mathrm{P} 1}=2.34$

$\mathrm{r}_{\mathrm{A} 12-\mathrm{P} 2}=2.31$

$\mathrm{r}_{\mathrm{A} 13-\mathrm{P} 1}=2.42$

$\mathrm{r}_{\mathrm{A} 11-\mathrm{P} 2}=2.35$

$\mathrm{r}_{\mathrm{A} 13-\mathrm{A} 11}=2.85$

$r_{A 11-P 1}=2.45$

$\mathrm{a}_{\mathrm{A} 13-\mathrm{P} 1-\mathrm{A} 12}=124.3$

$\mathrm{d}_{\mathrm{A} 11-\mathrm{P}-\mathrm{A} 12-\mathrm{P} 1}=55.6$
(27)

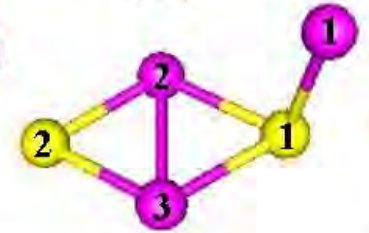

$\mathrm{r}_{\mathrm{P} 1-\mathrm{A} 11}=2.40$

$\mathrm{r}_{\mathrm{P} 1-\mathrm{A} 12}=\mathrm{r}_{\mathrm{A} 13-\mathrm{P} 1}=2.36$

$\mathrm{r}_{\mathrm{A} 12-\mathrm{P} 2}=\mathrm{r}_{\mathrm{A} 13-\mathrm{P} 2}=2.26$

$\mathrm{r}_{\mathrm{A} 12-\mathrm{A} 13}=2.56$

$\mathrm{a}_{\mathrm{P} 2-\mathrm{A} 12-\mathrm{P} 1}=112.4$

$\mathrm{d}_{\text {Al1-P1-A13-P2 }}=98.7$

(31)

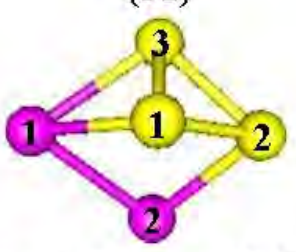

$\mathrm{r}_{\mathrm{P} 2-\mathrm{P} 3}=\mathrm{r}_{\mathrm{P} 1-\mathrm{P} 2}=2.20$

$\mathrm{r}_{\mathrm{P} 1-\mathrm{P} 3}=2.29$

$\mathrm{r}_{\mathrm{A} 1-\mathrm{A} 1}=2.54$

$\mathrm{r}_{\mathrm{A} 11-\mathrm{P} 3}=2.48$

$r_{\mathrm{Al2}-\mathrm{P} 2}=2.36$

$\mathrm{a}_{\mathrm{A} 12-\mathrm{P} 2-\mathrm{P} 3}=78.3$

$\mathrm{d}_{\mathrm{A} 12-\mathrm{P} 2-\mathrm{P} 3-\mathrm{P} 1}=64.4$

(35)
(28)

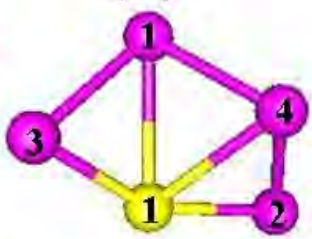

$\mathrm{r}_{\mathrm{A} 11-\mathrm{A} 13}=\mathrm{r}_{\mathrm{A} 112-\mathrm{A} 14}=2.1$

$r_{\mathrm{P} 1-\mathrm{A} 13}=r_{\mathrm{P} 1-\mathrm{A} 12}=2.34$

$r_{\text {A11-A.14 }}=2.55$

$\mathrm{r}_{\mathrm{P} 1-\mathrm{A} 13}=2.34$

$r_{\text {P1-A.11 }}=2.67$

$\mathrm{a}_{\mathrm{A} 13-\mathrm{P} 1-\mathrm{A} 12}=153.3$

$\mathrm{d}_{\mathrm{A} 14-\mathrm{A} 12-\mathrm{P} 1-\mathrm{A} 13}=26.6$

(32)

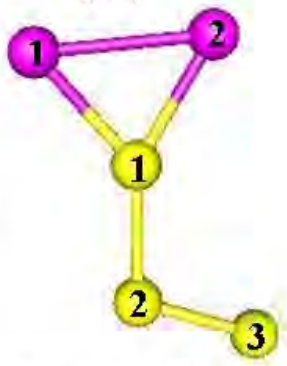

$\mathrm{r}_{\mathrm{P} 1-\mathrm{P} 2}=2.21$

$\mathrm{r}_{\mathrm{P} 2-\mathrm{P} 3}=2.00$

$\mathrm{r}_{\mathrm{Al}-\mathrm{P}}=2.33$

$\mathrm{r}_{\mathrm{A} 1-\mathrm{A} 1 \mathrm{1}}=2.74$

$\mathrm{a}_{\mathrm{A} 12-\mathrm{P} 1-\mathrm{P} 2}=148.6$

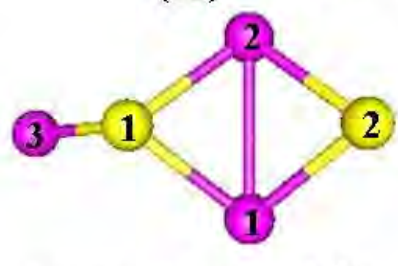

$\mathrm{r}_{\text {A12-P1 }}=\mathrm{r}_{\text {A11-P2 }}=2.36$

$\mathrm{r}_{\mathrm{A} 11-\mathrm{P} 1}=\mathrm{r}_{\mathrm{A} 12-\mathrm{P} 1}=2.37$

$\mathrm{r}_{\mathrm{P} 1-\mathrm{A} 13}=2.35$

$\mathrm{r}_{\mathrm{A} 1-\mathrm{Al}}=2.83$

$a_{A 12-\mathrm{P} 1-\mathrm{A} 13}=116.3$

$\mathrm{d}_{\mathrm{P} 2 \text {-A.11-P1-A13 }}=139.3$

Figura 5.6. Geometrías de equilibrio de los pentámeros (25) a (35). Las distancias (r) están expresadas en $\AA$ y los ángulos planos (a) y diedros (d), en ${ }^{\circ}$. 
En las Figuras 5.4 a 5.6 se puede ver que las distancias interatómicas Al-Al de los pentámeros se encuentran entre los valores 2.54 y $2.63 \AA$; las uniones Al-P entre 2.20 y $2.48 \AA$ y las uniones P-P entre 2.03 y $2.22 \AA$. Para un agregado de estructura similar a (17), Guo y colaboradores $^{88}$ informan distancias de enlace Al-P de $2.44 \AA$ y P-P de $2.3 \AA \AA$. Los mismos autores describen un agregado con geometría similar a (28), aunque de estado electrónico doblete (Tabla 5.6), con distancias de enlace A11-A14 de $2.63 \AA$, Al4-A12 de $2.82 \AA$, Al4-P1 de $2.46 \AA$ y Al2-P1 de $2.41 \AA ̊$.

Tabla 5.7. Modos normales de vibración $\left(\omega_{\mathrm{e}}\right)$ de los pentámeros, en $\mathrm{cm}^{-1}$. * indica un modo doblemente degenerado.

\begin{tabular}{c|c} 
Agregado & $\boldsymbol{\omega}_{\mathbf{e}}$ \\
\hline $\mathbf{( 1 4 )}$ & $56,95,103,196,228,282,330,384,481$ \\
\hline $\mathbf{( 1 5 )}$ & $57,74,150,204,234,288,268,494,585$ \\
\hline $\mathbf{( 1 6 )}$ & $46,107,130,191,241,303,319,423,455$ \\
\hline $\mathbf{( 1 7 )}$ & $140,177,220,261,283,327,349,464,470$ \\
\hline & $177,304,317,343,386,492^{88}$ \\
\hline $\mathbf{( 1 8 )}$ & $52,97,179,219,316,353,408,445,539$ \\
\hline $\mathbf{( 1 9 )}$ & $74,125,172,236,341,348,406,450,602$ \\
\hline $\mathbf{( 2 0 )}$ & $83,117,169,231,289,315,388,409,558$ \\
\hline $\mathbf{( 2 1 )}$ & $84,85,174,215,323,343,458,473,521$ \\
\hline $\mathbf{( 2 2 )}$ & $45^{*}, 115,130,300,349,378,485,628$ \\
\hline $\mathbf{( 2 3 )}$ & $74,75,114,255,257,354,372,600,636$ \\
\hline $\mathbf{( 2 4 )}$ & $68,92,160,193,263,358,384,439,584$ \\
\hline $\mathbf{( 2 5 )}$ & $46,89,127,227,283,323,453,556,596$ \\
\hline $\mathbf{( 2 6 )}$ & $45,99,141,199,219,272,317,357,411$ \\
\hline $\mathbf{( 2 7})$ & $44,47,156,212,311,368,370,423,506$ \\
\hline $\mathbf{( 2 8 )}$ & $40,76,84,207,238,242,304,318,401$ \\
\hline & $31,70,107,132,182,271,284,354,397^{88}$ \\
\hline $\mathbf{( 2 9 )}$ & $73,111,166,222,262,319,363,461,557$ \\
\hline $\mathbf{( 3 0 )}$ & $59,61,129,211,243,309,335,406,445$ \\
\hline $\mathbf{( 3 1 )}$ & $97,215,247,278,336,361,379,411,519$ \\
\hline $\mathbf{( 3 2 )}$ & $30,45,46,139,201,292,387,415,607$ \\
\hline $\mathbf{( 3 3 )}$ & $64,129,138,156,203,278,325,348,494$ \\
\hline $\mathbf{( 3 4 )}$ & $43,111,138,221,245,306,350,358,441$ \\
\hline $\mathbf{( 3 5 )}$ & $29,50,85,223,309,346,356,376,417$
\end{tabular}

En los pentámeros A1/P se presentan movimientos de tipo twisting, wagging y scissoring en frecuencias bajas; en frecuencias intermedias se presentan estiramientos simétricos y asimétricos Al-Al y Al-P y a más de $406 \mathrm{~cm}^{-1}$, estiramientos P-P, muchas veces acoplados con estiramientos Al-P. Similares resultados se reportan en la referencia 88 para los agregados (17) y (28).

Análisis de cargas derivadas del potencial electrostático y de la densidad de espín

Los parámetros presentados en esta sección se calcularon con el mismo nivel de teoría utilizado para encontrar las geometrías de equilibrio y demás parámetros anteriormente mostrados. En las Tablas 5.8 a 5.10 se muestran las cargas ESP junto con la densidad de espín. En las Figuras 5.7 a 5.10 se muestran las representaciones gráficas de la densidad de espín. 
Tabla 5.8. Análisis de cargas ESP (en unidades de $|e|$ ) y densidades de espín entre paréntesis, en u.a., del dímero y los trímeros. Los átomos se numeran de izquierda a derecha.

\begin{tabular}{c|c|c|c|c}
$\#$ & Al1 & Al2 & P1 & P2 \\
\hline $\mathbf{( 1 )}$ & $0.34(0.53)$ & & $-0.34(1.47)$ & \\
\hline $\mathbf{( 2 )}$ & $0.10(1.19)$ & $0.32(0.49)$ & $-0.42(1.32)$ & \\
\hline $\mathbf{( 3 )}$ & $0.28(0.12)$ & & $-0.15(0.19)$ & $-0.13(0.72)$ \\
\hline $\mathbf{( 4 )}$ & $0.26(0.29)$ & $0.26(0.29)$ & $-0.51(0.44)$ & \\
\hline $\mathbf{( 5 )}$ & $0.51(-0.03)$ & & $-0.25(1.51)$ & $-0.25(1.51)$
\end{tabular}

Tabla 5.9. Análisis de cargas ESP (en unidades de |e|) y densidades de espín entre paréntesis, en u.a., de los tetrámeros. Los átomos se numeran de izquierda a derecha, o como se muestra

\begin{tabular}{c|c|c|c|c|c|c}
$\#$ & Al1 & Al2 & Al3 & P1 & P2 & P3 \\
\hline $\mathbf{( 6 )}$ & $0.18(0.70)$ & $0.21(0.00)$ & & $-0.20(0.39)$ & $-0.19(0.90)$ & \\
\hline $\mathbf{( 7 )}$ & $0.02(0.94)$ & $0.37(0.53)$ & $0.22(0.22)$ & $-0.60(0.32)$ & & \\
\hline $\mathbf{( 8 )}$ & 0.49 & & & -0.31 & 0.12 & -0.31 \\
\hline $\mathbf{( 9 )}$ & $0.50(0.02)$ & & & $-0.24(1.70)$ & $-0.13(0.16)$ & $-0.13(0.16)$ \\
\hline $\mathbf{( 1 0 )}$ & $0.21(0.12)$ & $0.58(-0.11)$ & & $-0.50(0.35)$ & $-0.29(1.64)$ & \\
\hline $\mathbf{( 1 1 )}$ & 0.17 & 0.17 & & -0.20 & -0.14 & \\
\hline $\mathbf{( 1 2 )}$ & 0.03 & 0.03 & 0.01 & -0.08 & & \\
\hline $\mathbf{( 1 3 )}$ & $0.32(0.38)$ & $0.33(0.38)$ & & $-0.33(0.62)$ & $-0.33(0.62)$ &
\end{tabular}


Tabla 5.10. Análisis de cargas ESP (en unidades de $|e|$ ) y densidades de espín, entre paréntesis y en u.a., de los pentámeros. Los átomos se numeran de izquierda a derecha o como muestran las Figuras 5.4 a 5.6.

\begin{tabular}{|c|c|c|c|c|c|c|c|c|}
\hline$\#$ & Al1 & $\mathrm{A} 12$ & Al3 & Al4 & $\mathrm{P} 1$ & P2 & $\mathrm{P} 3$ & $\mathrm{P} 4$ \\
\hline (14) & $0.19(0.87)$ & $0.24(0.81)$ & $-0.04(1.28)$ & & $-0.21(0.55)$ & $-0.18(1.48)$ & & \\
\hline (15) & $0.27(-0.14)$ & $0.27(0.03)$ & & & $-0.03(0.01)$ & $-0.16(-0.19)$ & $-0.35(1.26)$ & \\
\hline (16) & $0.09(1.05)$ & $0.18(0.74)$ & & & $-0.02(0.15)$ & $-0.15(1.18)$ & $-0.10(1.87)$ & \\
\hline (17) & $0.33(0.41)$ & $0.33(0.41)$ & & & $-0.36(0.11)$ & $0.07(-0.07)$ & $-0.37(0.11)$ & \\
\hline (18) & $0.35(0.54)$ & & & & $-0.23(0.39)$ & $0.05(0.21)$ & $-0.08(0.12)$ & $-0.09(1.75)$ \\
\hline (19) & $0.34(0.60)$ & & & & $-0.20(0.70)$ & $0.14(-0.10)$ & $-0.20(0.70)$ & $-0.08(1.08)$ \\
\hline (20) & $0.56(0.11)$ & $0.37(0.80)$ & & & $-0.48(0.47)$ & $-0.19(0.74)$ & $-0.25(0.88)$ & \\
\hline (21) & $0.55(0.03)$ & & & & $-0.18(1.86)$ & $-0.20(0.65)$ & $0.03(-0.15)$ & $-0.20(0.65)$ \\
\hline (22) & $0.48(-0.02)$ & $0.29(0.28)$ & & & $-0.48(0.44)$ & $-0.10(0.17)$ & $-0.20(0.14)$ & \\
\hline (23) & $0.43(0.02)$ & & & & $-0.11(0.25)$ & $-0.11(0.24)$ & $-0.10(0.25)$ & $-0.11(0.25)$ \\
\hline (24) & $0.22(0.36)$ & $0.58(0.03)$ & $0.20(0.36)$ & & $-0.50(0.12)$ & $-0.50(0.12)$ & & \\
\hline (25) & $0.35(0.73)$ & $0.52(0.11)$ & & & $-0.54(0.12)$ & $-0.12(0.05)$ & $-0.20(0.01)$ & \\
\hline (26) & $0.23(0.55)$ & $0.15(0.71)$ & $0.13(0.33)$ & $-0.05(1.04)$ & $-0.45(0.36)$ & & & \\
\hline (27) & $0.19(0.02)$ & $0.49(0.52)$ & $0.49(0.52)$ & & $-0.51(0.01)$ & $-0.66(-0.04)$ & & \\
\hline (28) & $0.03(0.70)$ & $0.15(0.66)$ & $0.14(0.65)$ & $0.04(0.70)$ & $-0.36(0.30)$ & & & \\
\hline (29) & $0.33(-0.07)$ & $0.52(0.02)$ & & & $-0.18(0.39)$ & $-0.22(0.17)$ & $-0.45(0.49)$ & \\
\hline (30) & $0.24(0.84)$ & $0.24(0.84)$ & $-0.04(1.23)$ & & $-0.17(1.75)$ & $-0.27(0.34)$ & & \\
\hline (31) & $0.17(0.60)$ & $0.16(0.02)$ & & & $-0.15(0.04)$ & $-0.03(0.29)$ & $-0.15(0.04)$ & \\
\hline (32) & $0.13(0.79)$ & $0.16(0.79)$ & & & $-0.14(0.55)$ & $-0.06(0.08)$ & $-0.08(0.89)$ & \\
\hline (33) & $0.11(0.08)$ & $0.14(0.13)$ & $0.12(0.08)$ & & $-0.16(0.39)$ & $-0.21(0.33)$ & & \\
\hline (34) & $0.25(0.16)$ & $0.31(0.56)$ & $0.05(-0.01)$ & & $-0.24(0.07)$ & $-0.37(0.22)$ & & \\
\hline (35) & $0.31(0.73)$ & $0.31(0.73)$ & $0.17(0.13)$ & & $-0.37(0.35)$ & $-0.42(1.08)$ & & \\
\hline
\end{tabular}




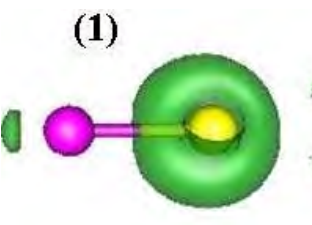

(4)

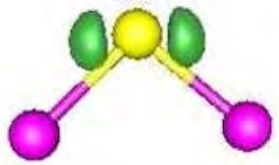

(2)

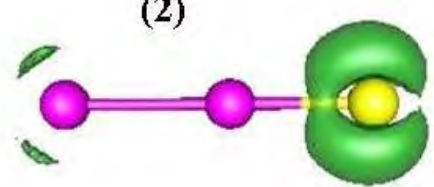

(3)

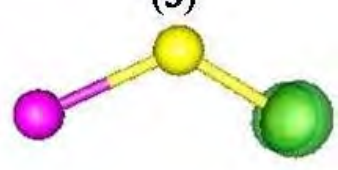

(5)

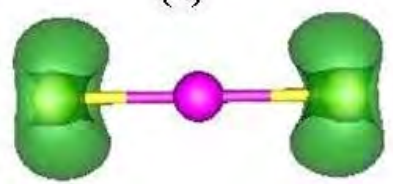

Figura 5.7. Densidades de espín del dímero y los trímeros. Las superficies se graficaron con un valor de isosuperficie de 0.015 u.a.

(6)
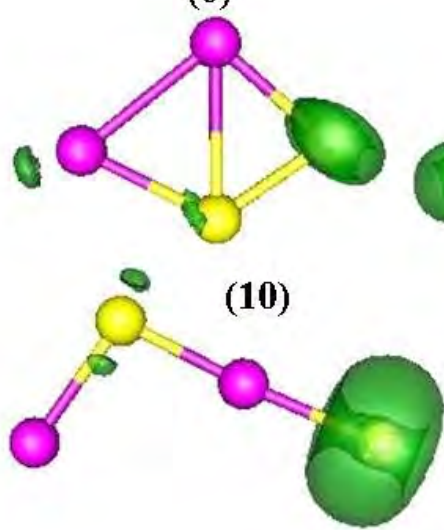

(7)
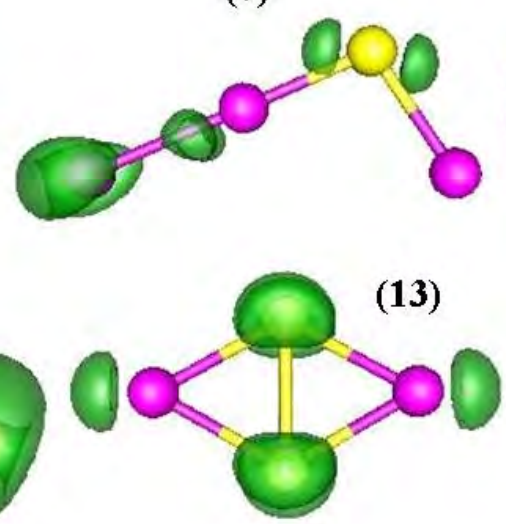

Figura 5.8. Densidades de espín de los tetrámeros de capa abierta. Las superficies se graficaron con un valor de isosuperficie de 0.015 u.a., con excepción del agregado (13), que se graficó con un valor de isosuperficie de 0.01 u.a. 


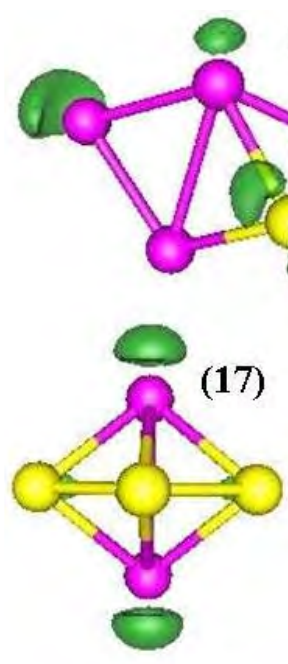

(20)
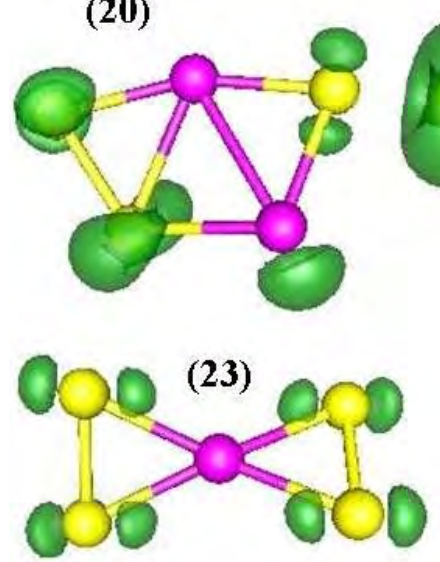

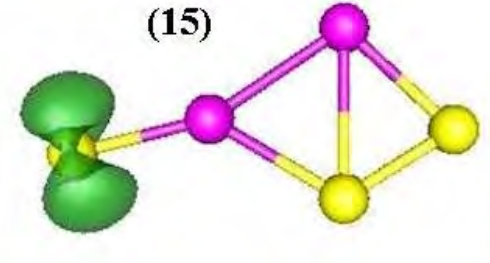

(18)

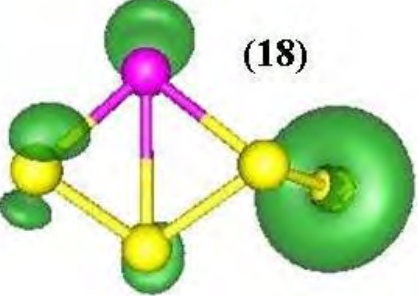

(21)

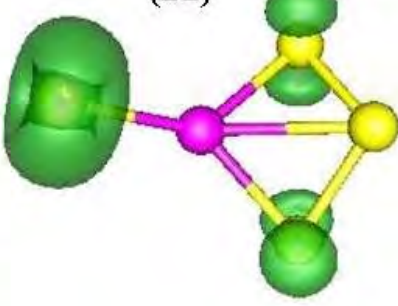

(24)

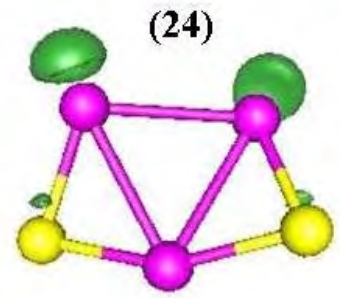

(19)
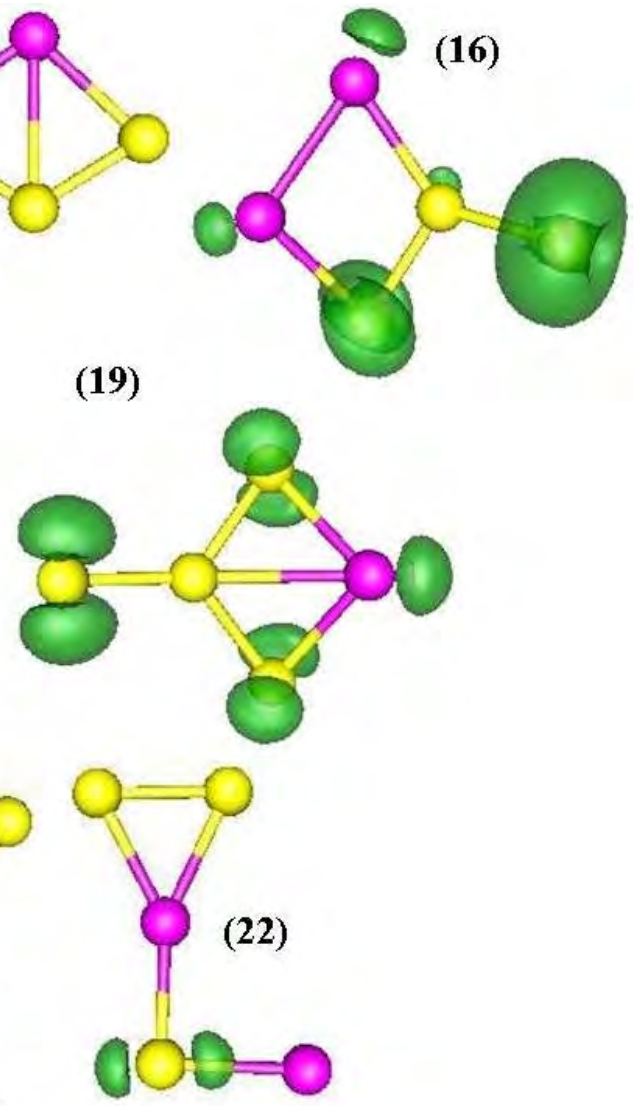

Figura 5.9. Densidades de espín de pentámeros (14) a (24). Las superficies se graficaron con un valor de isosuperficie de 0.015 u.a. 

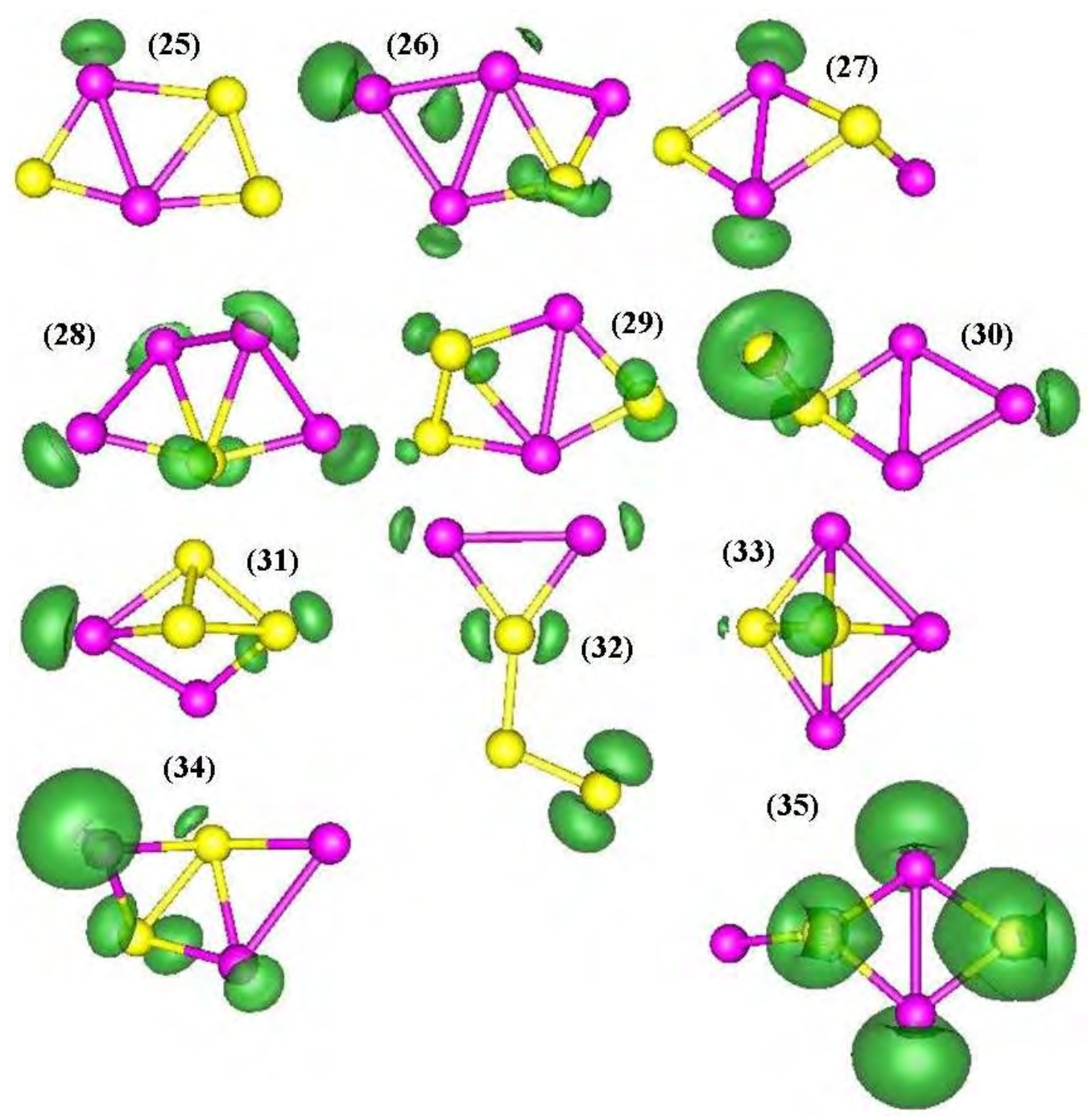

Figura 5.10. Densidades de espín de pentámeros (25) a (35). Las superficies se graficaron con un valor de isosuperficie de 0.015 u.a., con excepción de los agregados (32) y (33), que se graficaron con un valor de isosuperficie de 0.05 u.a.

\subsection{Potenciales electrostáticos moleculares}

En las Figuras 5.11 a 5.12 se muestran los gráficos de potenciales electrostáticos moleculares (MEP) calculados para todos los sistemas. El color azul muestra una zona susceptible de ataque electrofílico, mientras que el color rojo representa una zona probable de ataque nucleofílico, los colores verdes y amarillos indican zonas intermedias de valores negativos y positivos muy pequeños, respectivamente.

Además, en la Figura 5.11 se muestran también los valores de las cargas ESP calculadas para cada átomo correspondientes al dímero y los trímeros de $\mathrm{Al} / \mathrm{P}$, con la finalidad de mostrar la buena correspondencia existente entre ellos, véase Tablas 5.8 a 5.10 para los valores de las cargas ESP. Esto demuestra que las cargas ESP, por sí solas, indican correctamente las zonas de ataque nucleofílico y electrofílico. 
(2)

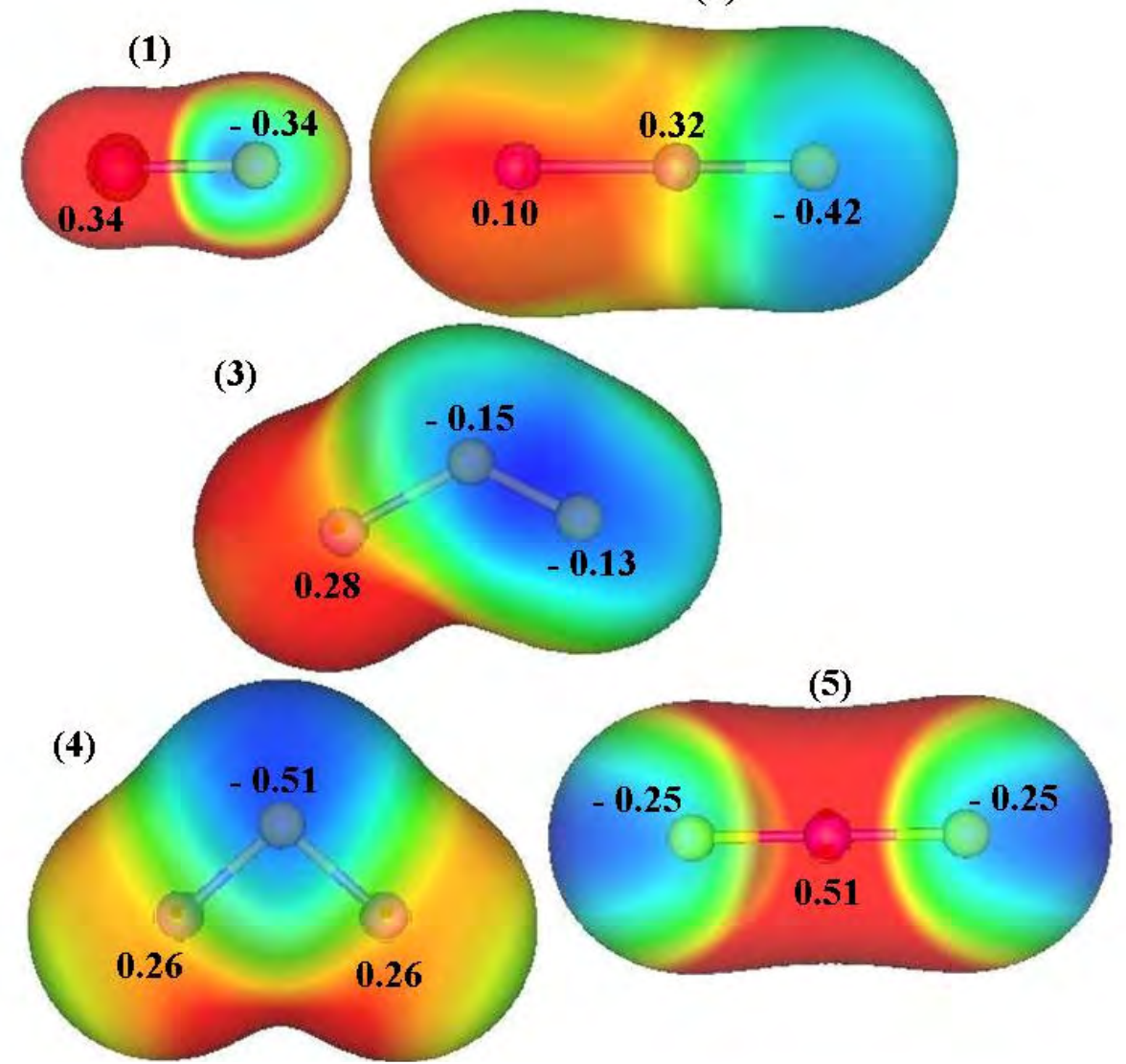

Figura 5.11. Superficies de potenciales electrostáticos moleculares del dímero y los trímeros. También se muestran las cargas ESP calculadas. Las superficies se graficaron con un valor de isosuperficie de la densidad electrónica de 0.001 u.a.

En la Figura 5.11, se observa que tanto en el dímero como en los trímeros las cargas ESP son negativas, correspondientes a zonas susceptibles de ataque electrofílico (MEP negativo), se sitúan sobre átomos de fósforo (Tabla 5.8) y corresponden a zonas donde se encuentran electrones desapareados, con excepción del P1 en el agregado (3) (Tabla 5.8, Figura 5.7). Además, las zonas susceptibles de ataque nucleofílico se concentran en los átomos de aluminio. 


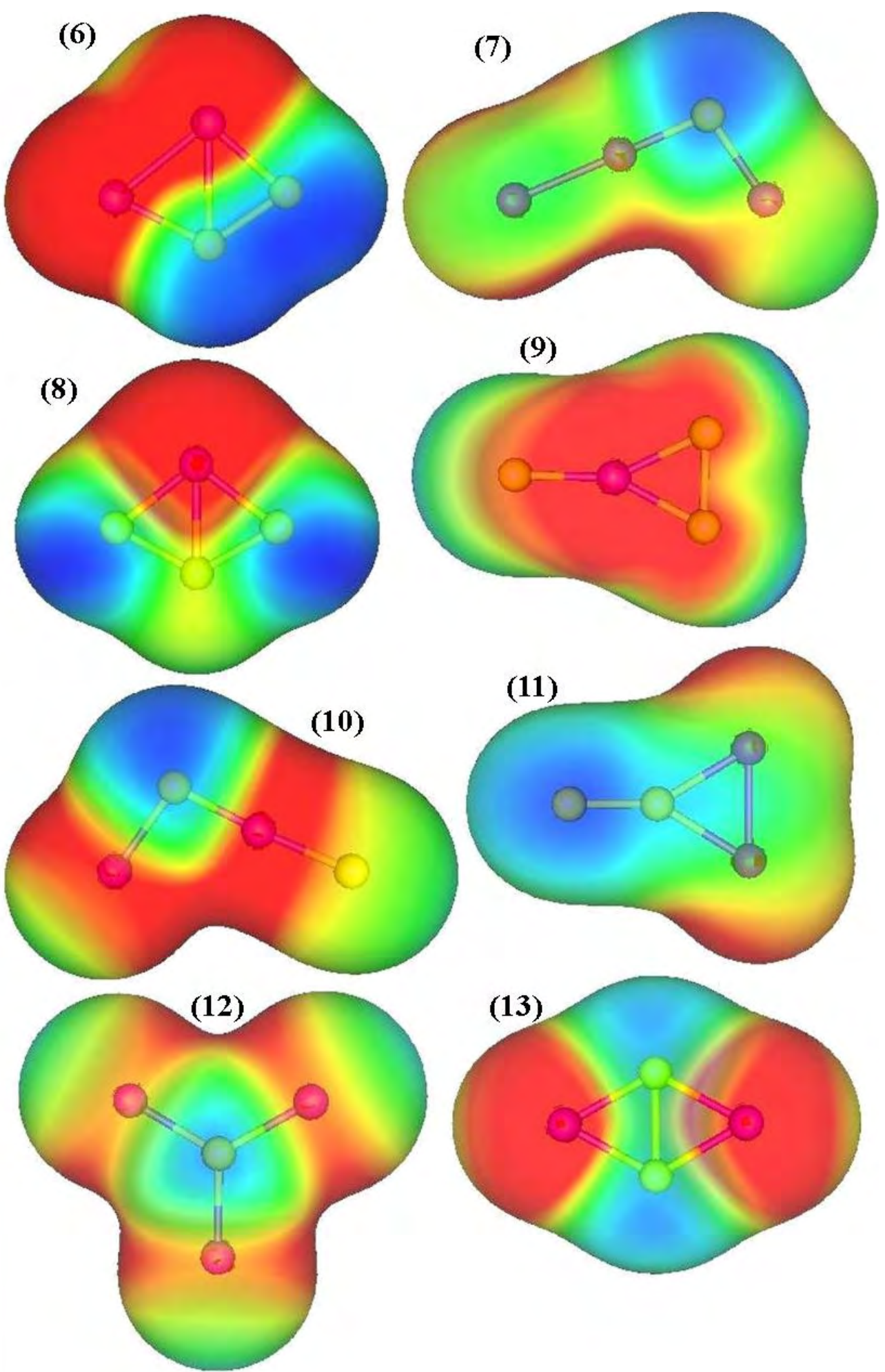

Figura 5.12. Superficies de potenciales electrostáticos moleculares para los tetrámeros. Las superficies se graficaron con un valor de isosuperficie de la densidad electrónica de $0.001 \mathrm{u}$.a.

En los tetrámeros continúa la tendencia de localizar las zonas susceptibles de ataque electrofílico en átomos de fósforo y zonas susceptibles de ataque nucleofílico en átomos de aluminio, con excepción del P2 en (8) (Figura 5.2, Tabla 5.9). Obsérvese que la 
representación gráfica de MEP del agregado (12) muestra valores muy pequeños, que se reflejan también en la Tabla 5.9. Con respecto a la localización de electrones desapareados, no se observa una tendencia de poseer densidades de espín altas en átomos con cargas ESP negativas (Figura 5.8, Tabla 5.9).

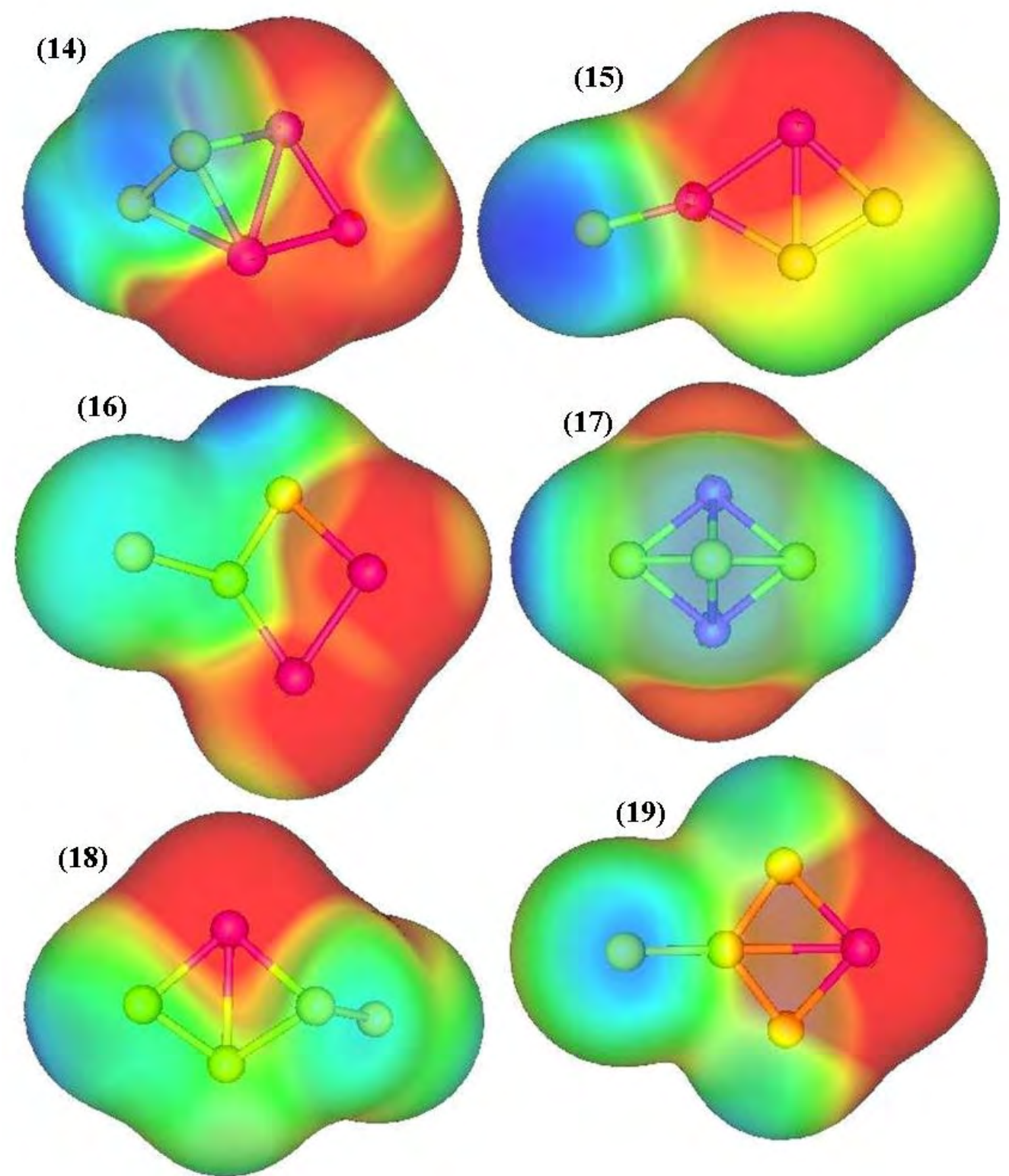

Figura 5.13. Superficies de potenciales electrostáticos moleculares para los pentámeros (14) a (19). Las superficies se graficaron con un valor de isosuperficie de la densidad electrónica de 0.001 u.a. 

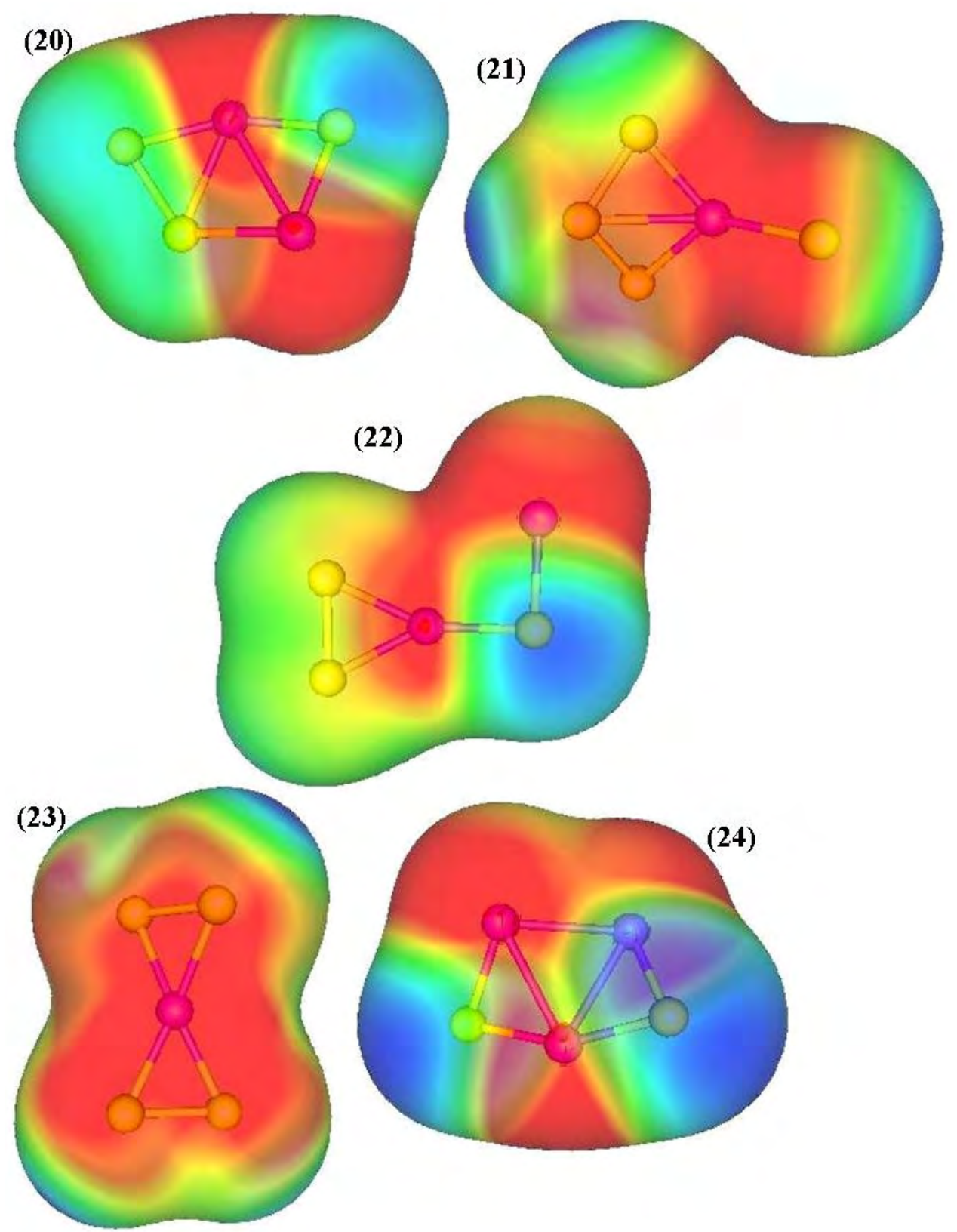

Figura 5.14. Superficies de potenciales electrostáticos moleculares para los pentámeros (20) a (24). Las superficies se graficaron con un valor de isosuperficie de la densidad electrónica de 0.001 u.a.

Al observar la Tabla 5.10 y las Figuras 5.13 y 5.14, se evidencia que los pentámeros (14) a (24) también sitúan las zonas de ataque electrofílico en átomos de fósforo, con excepción del átomo Al3 en (14) (Figura 5.4), que muestra una carga ESP negativa muy pequeña y los átomos $\mathrm{P} 2$ en (17), (18) y (19), que muestran cargas ESP negativas muy pequeñas. Por otro lado, en los pentámeros (14) a (24) las zonas de MEP negativo no siempre son zonas donde se presentan electrones desapareados (Figura 5.9). 

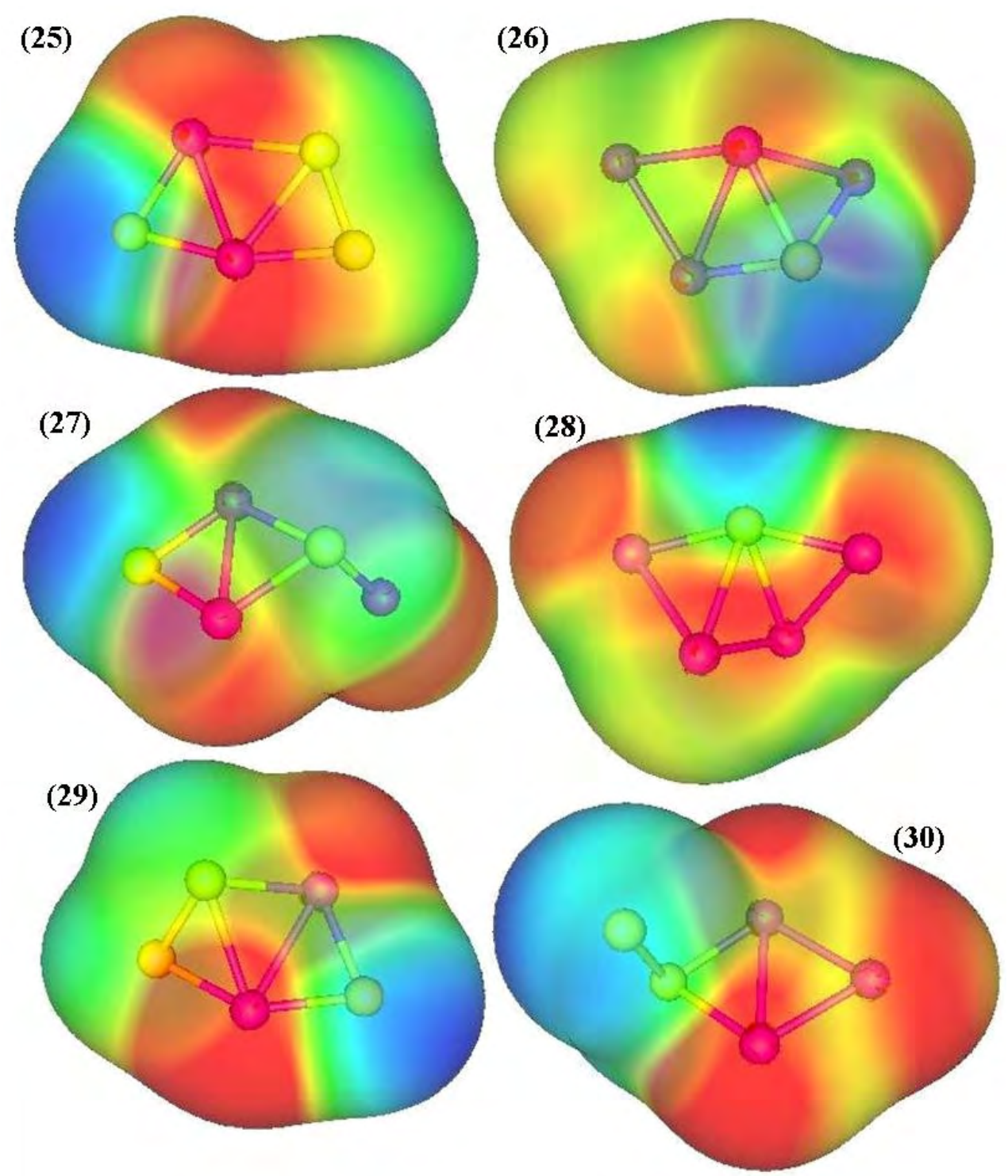

Figura 5.15. Superficies de potenciales electrostáticos moleculares para los pentámeros (25) a (30). Las superficies se graficaron con un valor de isosuperficie de la densidad electrónica de 0.001 u.a. 


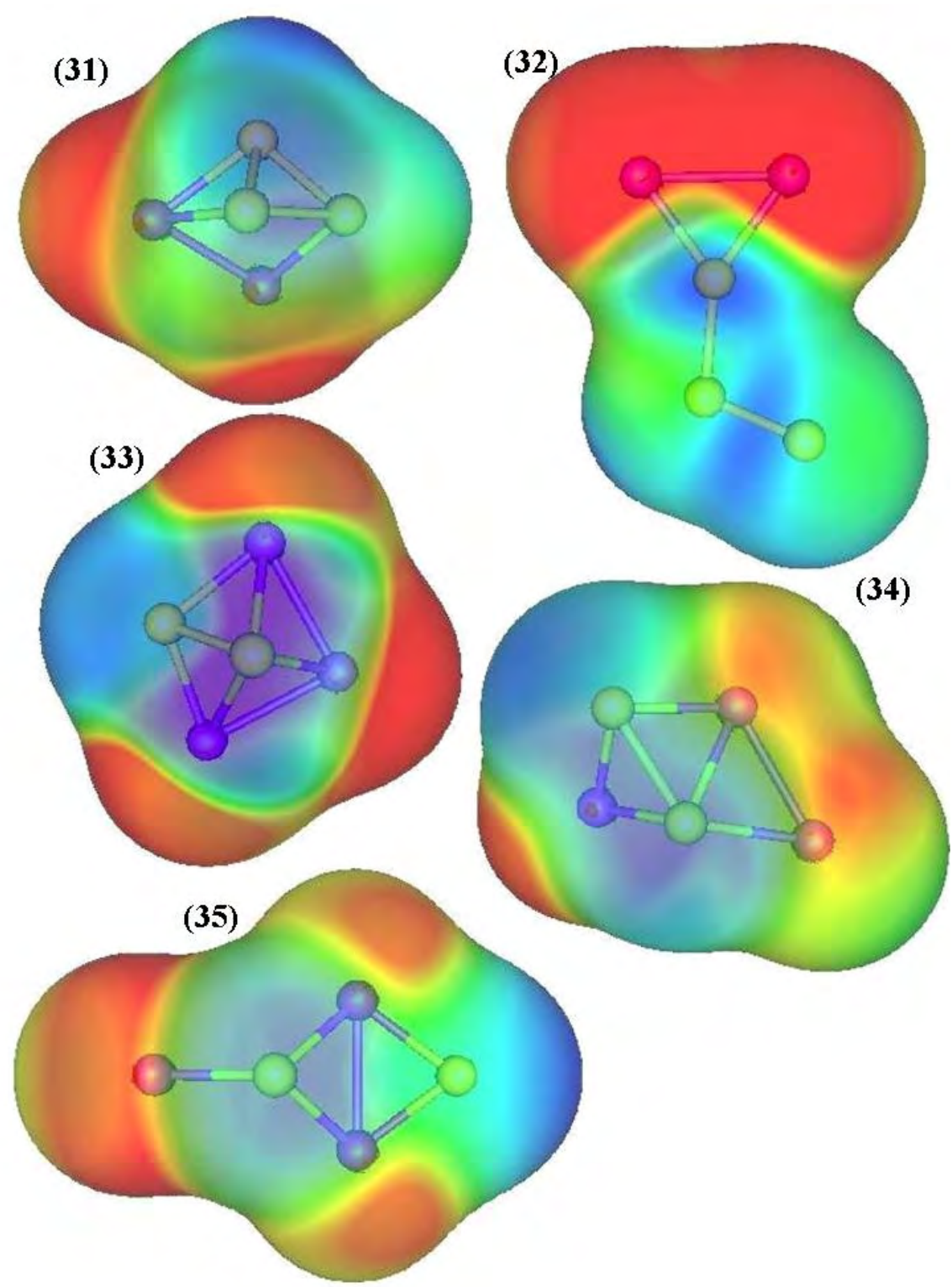

Figura 5.16. Superficies de potenciales electrostáticos moleculares para los pentámeros (31) a (35). Las superficies se graficaron con un valor de isosuperficie de la densidad electrónica de 0.001 u.a.

Con respecto a los pentámeros (25) a (35), se observa que se cumple la tendencia de ubicar las zonas susceptibles de ataque electrofílico sobre átomos de fósforo y las zonas susceptibles de ataque nucleofílico sobre átomos de aluminio (Tabla 5.10, Figuras 5.15 y 5.16). Constituyen la excepción el Al4 de (26) (Figura 5.6) y Al3 de (21). Asimismo, los pentámeros (25) a (33) tampoco sitúan en todos los casos los electrones desapareados sobre átomos de fósforo (Tabla 5.10, Figura 5.10).

\subsection{Patrones de crecimiento}

En las Figuras 5.17 a 5.19 se muestran los patrones de crecimiento obtenidos. Estos patrones se obtienen a partir del dímero (1) agregando átomos de $\mathrm{P}$ y de $\mathrm{Al}$ en todos los sitios posibles 
y siguiendo el camino de aumento de la energía de atomización. No se obtuvieron tetrámeros estables construidos a partir del trímero (2), por lo que se considera que este agregado no presenta patrón de crecimiento. Se plantea el patrón de crecimiento más probable para agregados binarios de $\mathrm{Al} / \mathrm{P}$.

En las Figuras 5.17.a, 5.17.b y 5.19.a se exponen los patrones de crecimiento que involucran al trímero (3).

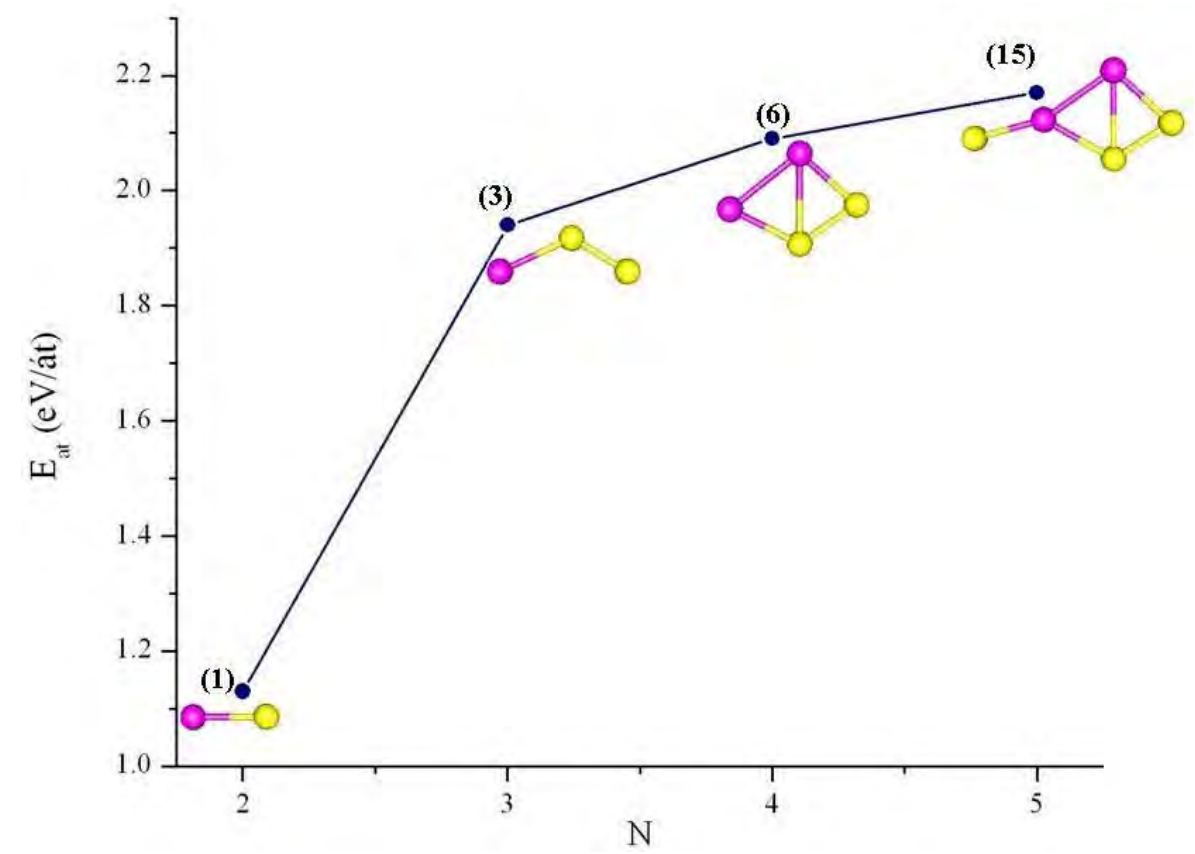

Figura 5.17.a Patrón de crecimiento del trímero (3) y el tetrámero (6).

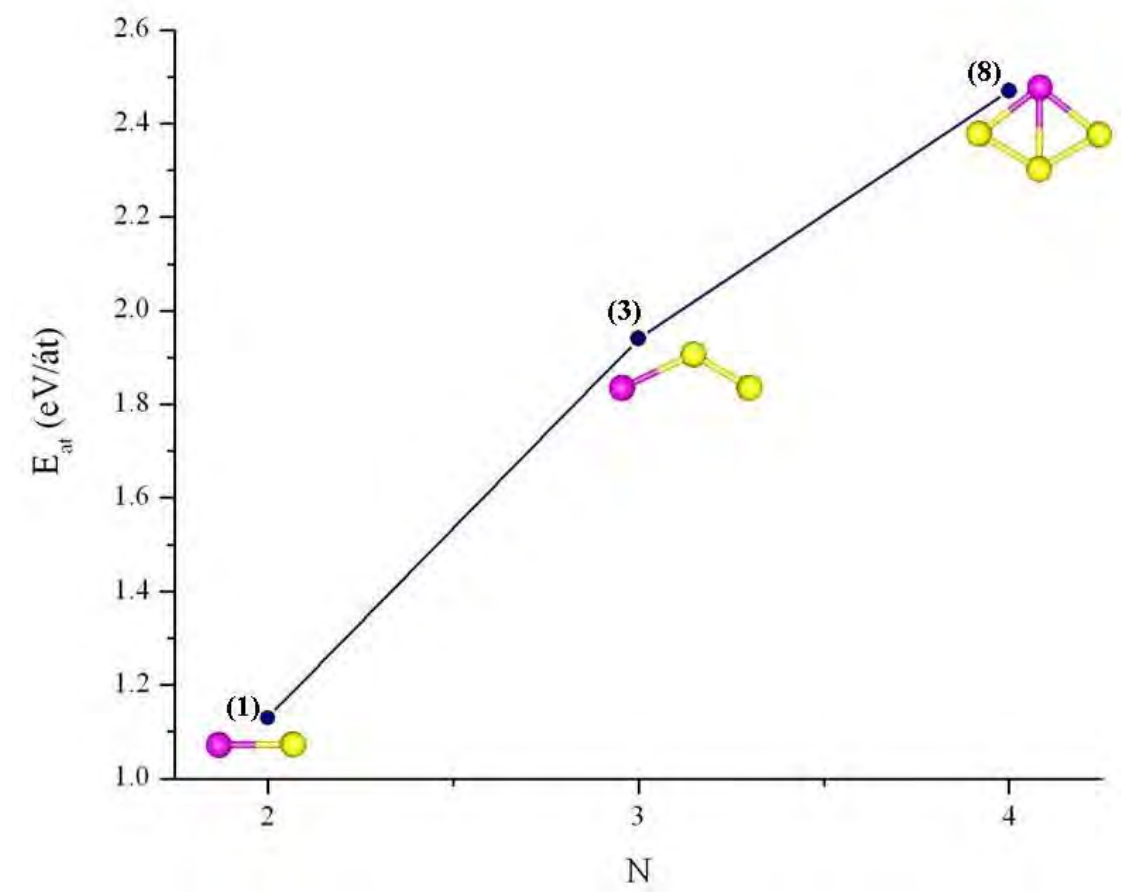

Figura 5.17.b Patrón de crecimiento del trímero (3) y el tetrámero (8). 
En los patrones de crecimiento del trímero (3) (Figuras 5.17.a y 5.17.b) se observa que para la obtención de (3), un átomo de fósforo ataca una zona susceptible de ataque electrofílico en el dímero Al-P, el átomo P1 (Tabla 5.8, Figuras 5.1 y 5.11). También se observa que, a pesar de que sea más favorable la obtención de (8) sobre (6), el primero no resulta luego en un pentámero de mayor energía de atomización. Para obtener (6) un átomo de Al se une a Al1 y P2 de (3), atacando a la vez tanto una zona susceptible de ataque nucleofílico, como una zona de ataque electrofílico en el trímero, respectivamente (Tabla 5.8 y Figura 5.11). Para la obtención de (15) un átomo de $\mathrm{P}$ ataca una zona susceptible de ataque nucleofílico (Tabla 5.9 y Figura 5.12) y electrones desapareados en (6), el átomo All (Figura 5.2 y 5.8).

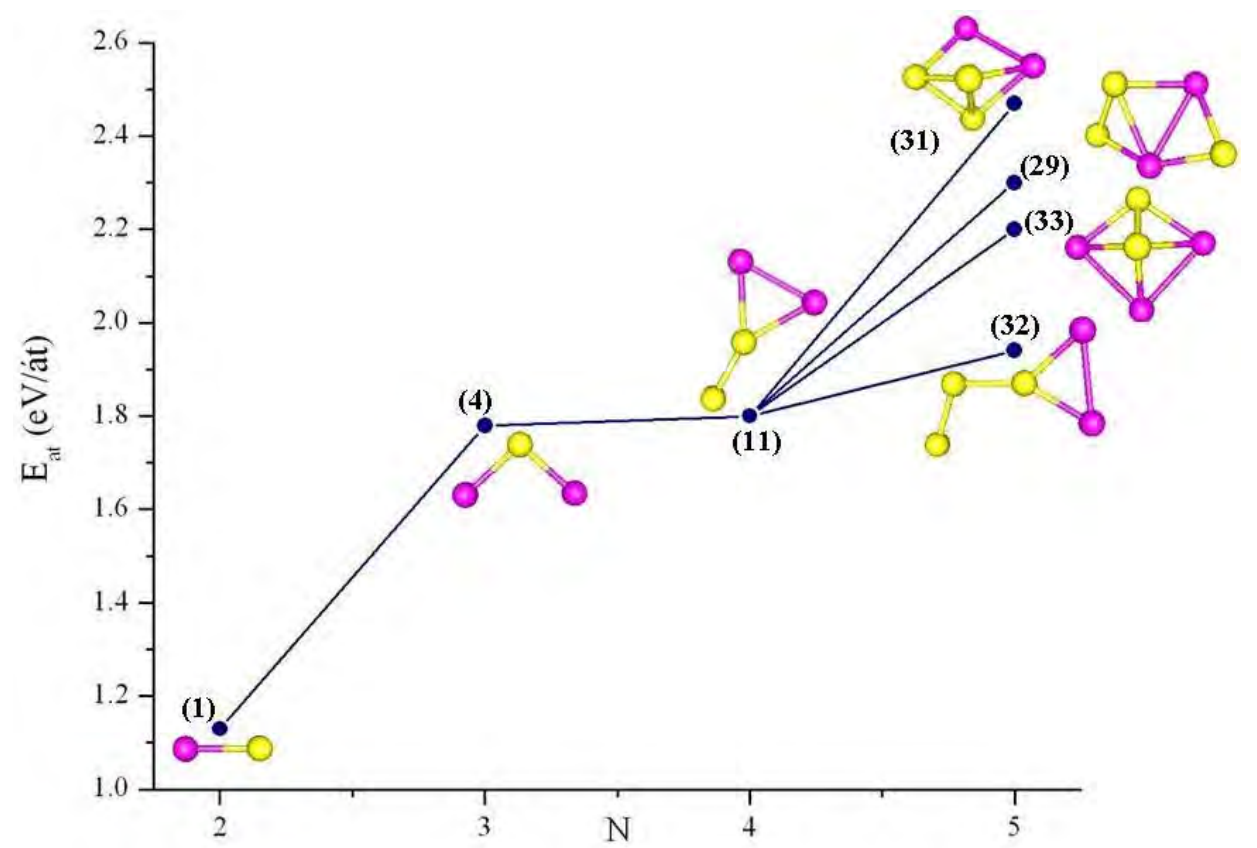

Figura 5.18.a Patrón de crecimiento del trímero (4) y el tetrámero (11).

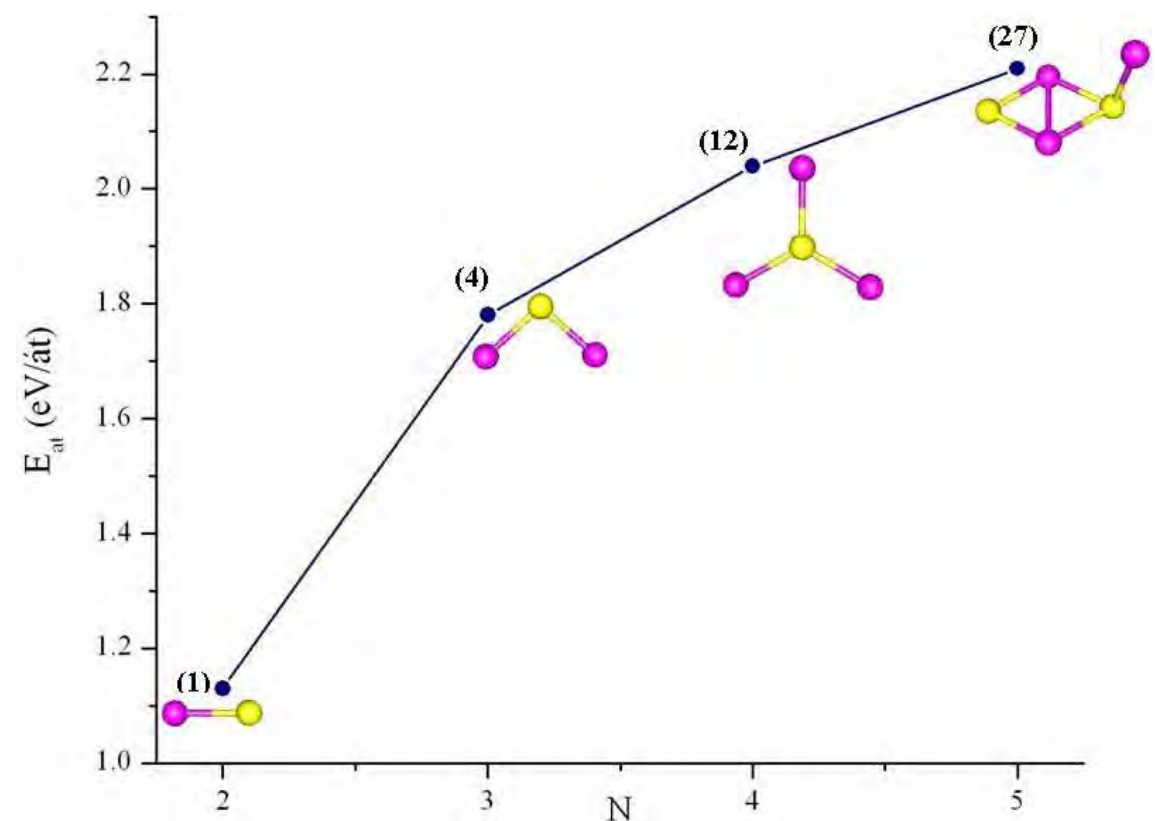

Figura 5.18.b Patrón de crecimiento del trímero (4) y el tetrámero (12). 
En las Figuras 5.18.a y 5.18.b se muestran los patrones de crecimiento obtenidos para el trímero (4). Para la obtención de este trímero, un átomo de $\mathrm{Al}$ ataca en una zona susceptible de ataque electrofílico y electrones desapareados del dímero (1) (Tabla 5.8, Figuras 5.7 y 5.11) y se une a P1. Siguiendo el camino de crecimiento de la Figura 5.18.a, para la obtención del tetrámero (11) un átomo de fósforo deberá atacar la zona probable de ataque electrofílico en P1 de (4), a diferencia de lo mostrado en la Figura 5.18.b, donde un átomo de aluminio ataca esta zona, resultando en un tetrámero de mayor energía de atomización, el agregado (12). Con respecto a la obtención de los pentámeros en el primer crecimiento propuesto de (4), se observa que se obtiene un pentámero con mayor energía de atomización si un átomo de fósforo se une al átomo de $\mathrm{P}$ del tetrámero (11) que posee carga ESP más negativa, esto es P1 (Tabla 5.8 y Figuras 5.2 y 5.12). Por otro lado, la obtención del agregado (29) se produce por la unión de un átomo de fósforo a una zona susceptible de ataque nucleofílico; la formación de (33) se realiza cuando un átomo de aluminio ataca una zona susceptible de ataque electrofílico; y para obtener (32), un átomo de fósforo ataca electrofílicamente en P2 de (11). Otro patrón de crecimiento del trímero (4) se muestra en la Figura 5.19.b.

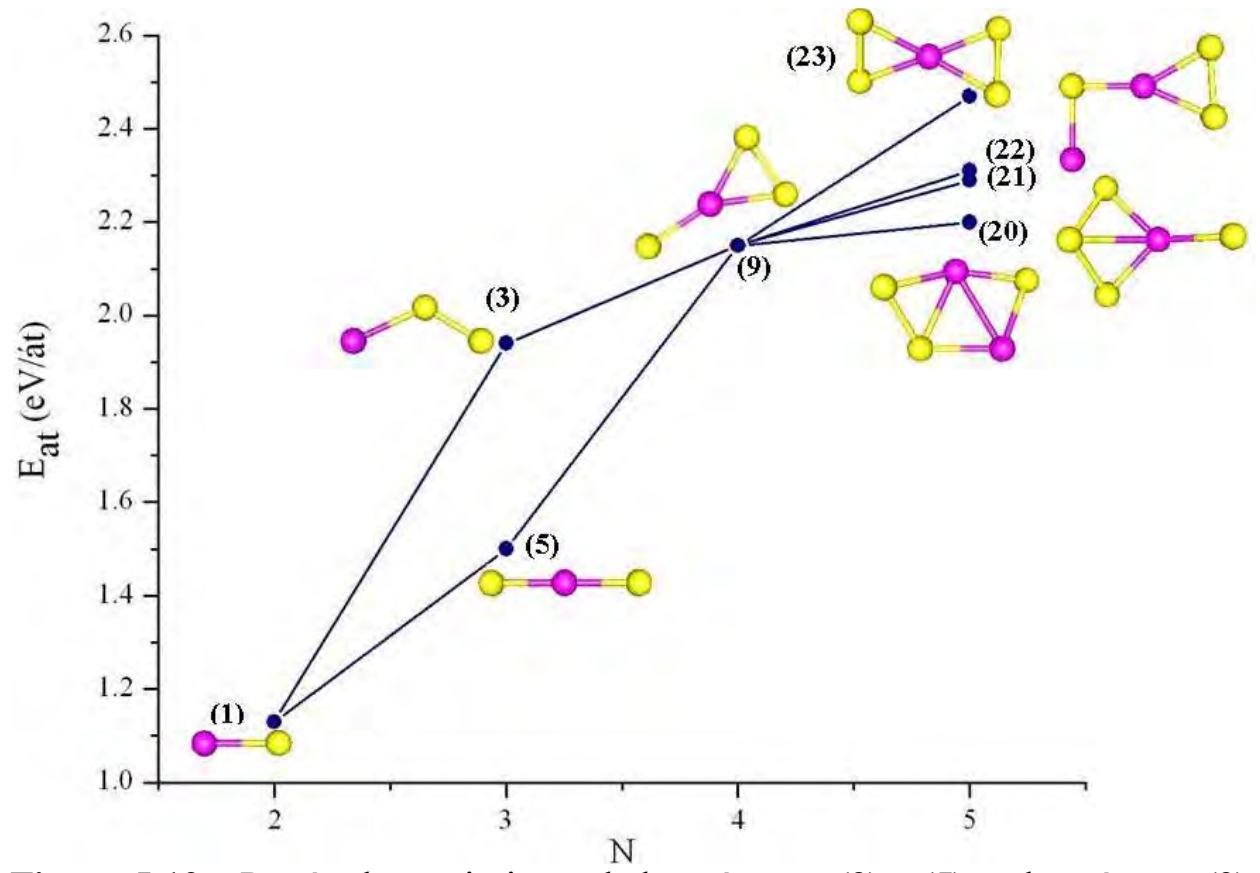

Figura 5.19.a Patrón de crecimiento de los trímeros (3) y (5) y el tetrámero (9).

Se observa en la Figura 5.19.a que el tetrámero (9) se puede obtener tanto a partir del trímero (5) como a partir del agregado (3), siendo éste último camino más favorable, debido a que posee mayor $E_{\text {at }}$. Para la obtención de (9) por la vía menos favorable, un átomo de fósforo ataca una zona probable de ataque electrofílico, P2 de (5), y luego se reacomoda la estructura uniéndose con Al1. Por otro lado, para la obtención del tetrámero por la vía más favorable, un átomo de fósforo ataca también una zona susceptible de ataque nucleofílico, Al1 en (3), aunque de menor carga ESP (Figura 5.1, Tabla 5.8) y mayor densidad de espín. Con respecto a la obtención de los pentámeros, resulta más favorable unir un átomo de fósforo al átomo de fósforo de carga ESP más negativa en (9) con electrones desapareados (átomo P1, Tabla 5.9 y Figuras 5.2 y 5.8) que las otras posibilidades: un átomo de aluminio atacando a la zona más probable de ataque electrofílico (obtención de agregado (22)), un átomo de $\mathrm{P}$ en una zona de ataque electrofílico de menor carga ESP (obtención de agregado (21)), o un átomo de aluminio en una zona de ataque nucleofílico (obtención de agregado (20)). Asimismo, el 
número de uniones es mayor en el pentámero obtenido por el patrón de crecimiento más favorable.

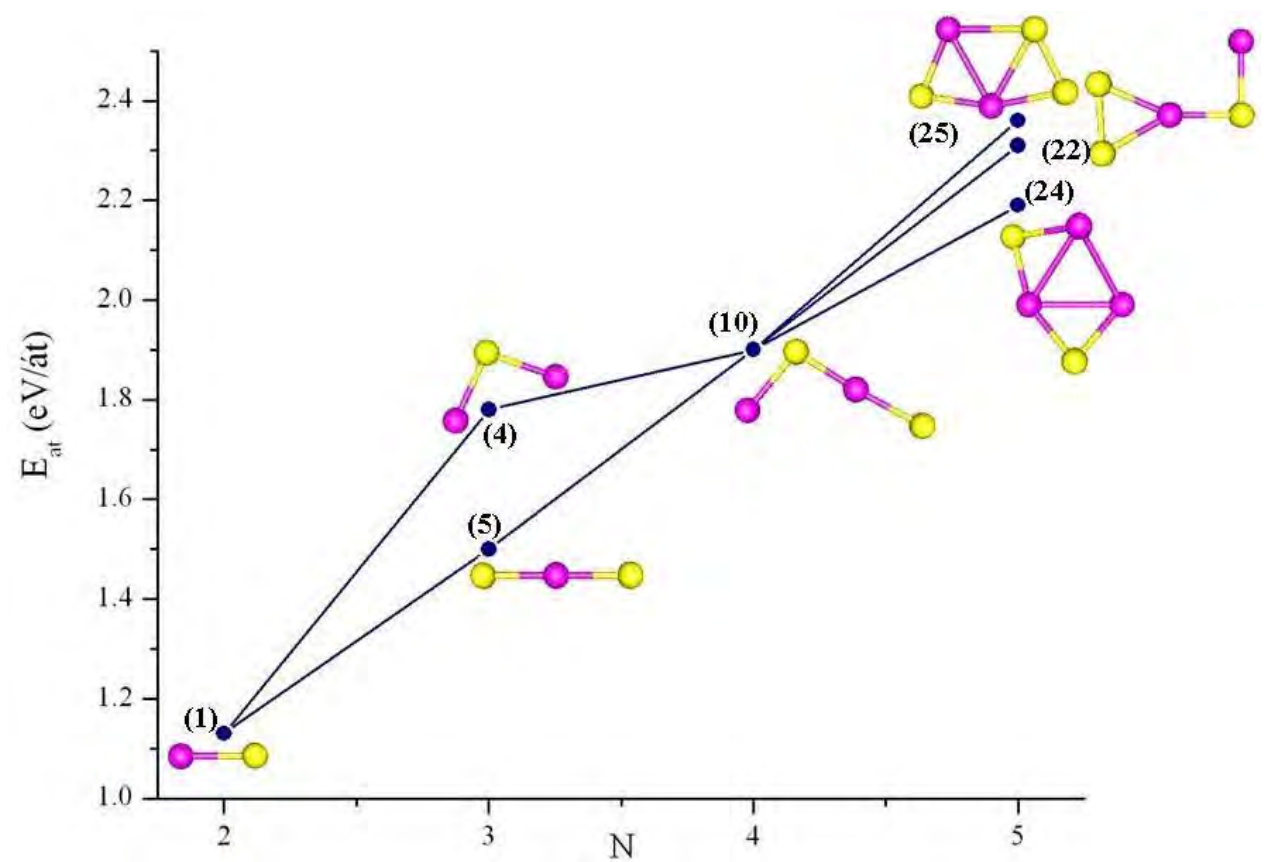

Figura 5.19.b Patrón de crecimiento de los trímeros (4) y (5) y tetrámero (10).

En la Figura 5.19.b se observa que la obtención de (10) a partir de (4) es más favorable, debido a que este último presenta mayor $\mathrm{E}_{\text {at }}$ que (5) (Tabla 5.1), uniéndose un átomo de $\mathrm{P}$ a una zona susceptible de ataque nucleofílico y electrones desapareados (Tabla 5.8). Con respecto a la obtención de los pentámeros de este patrón de crecimiento, se observa que resulta más favorable el ataque de un átomo de fósforo a una zona susceptible de ataque electrofílico para obtener (25), que el ataque de un átomo de fósforo uniéndose a Al2 de (10) para formar (22) (ataque nucleofílico), o la unión de Al en P2 de (10) para formar (24) (ataque electrofilico). En este caso también se observa una tendencia del sistema a favorecer patrones de crecimientos donde el pentámero obtenido tenga mayor cantidad de uniones. 


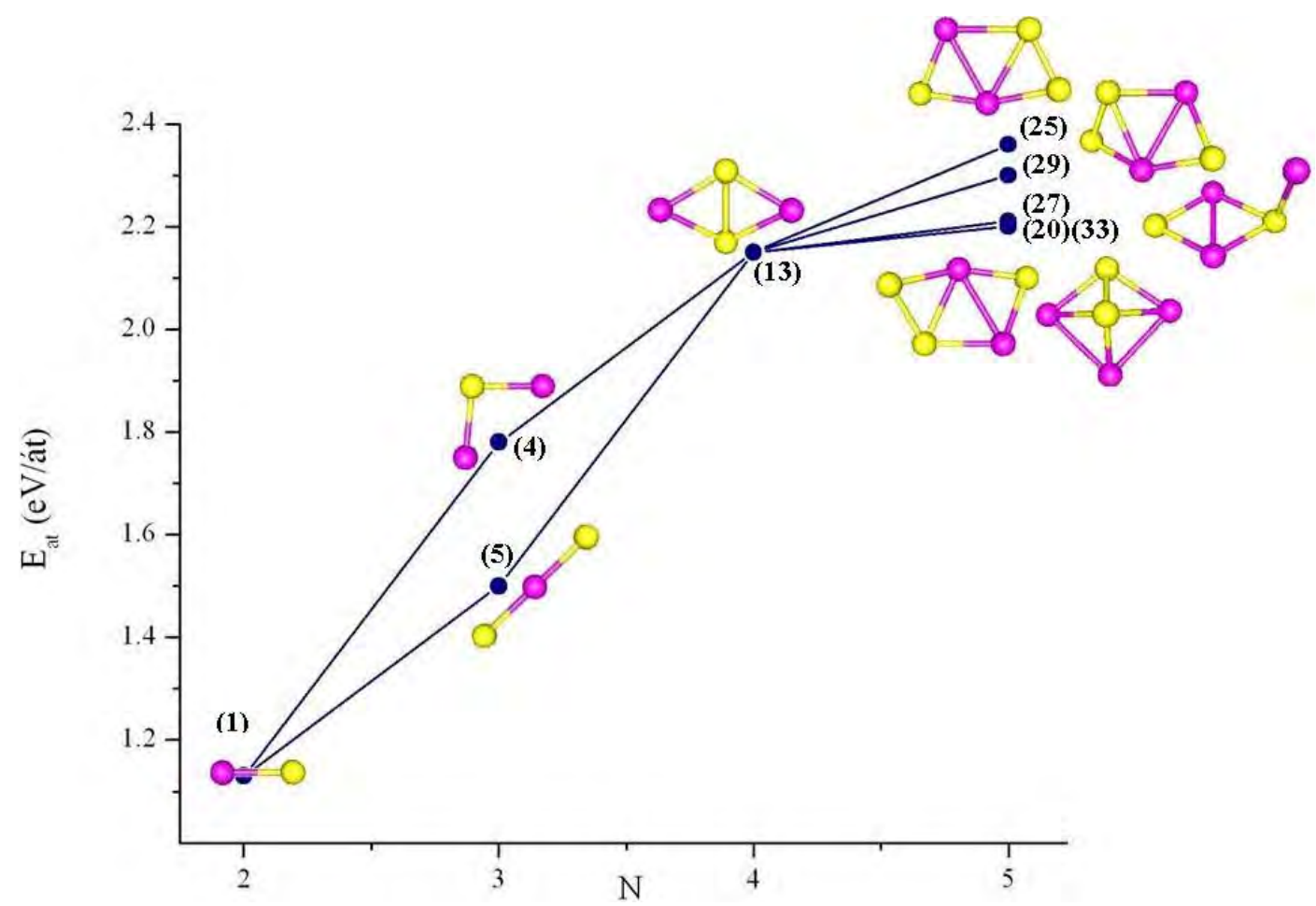

Figura 5.19.c Patrón de crecimiento de los trímeros (4) y (5) y tetrámero (13).

En la Figura 5.19.c se muestra el patrón de crecimiento del tetrámero romboidal alternado (13). En este patrón de crecimiento no se observa una tendencia del sistema a favorecer el crecimiento de pentámeros con mayor cantidad de uniones (Figuras 5.5 y 5.6). En este caso, resulta más favorable la unión de un átomo de fósforo a una zona susceptible de ataque nucleofílico en (4) (Tabla 5.8, Figura 5.11), para obtener (13). Para la obtención de los pentámeros, se evidencia una tendencia a favorecer la unión de átomos de fósforo al agregado (13) para obtener (25) y (29), más que la unión de átomos de aluminio para la obtención de (27). Asimismo, este patrón de crecimiento finaliza en el pentámero (25), que posee zonas susceptibles de ataque electrofílico sobre átomos de fósforos y una densidad de espín alta sobre un átomo de aluminio (Tabla 5.10).

Finalmente, los crecimientos más probables propuestos para cada trímero son:
(1) $\rightarrow$ (3) $\rightarrow$ (9) $\rightarrow$ (23)
$(1) \rightarrow(4) \rightarrow(12) \rightarrow(27)$
(Figura 5.19.a)
(Figura 5.17.b)
(1) $\rightarrow$ (5) $\rightarrow$ (9) $\rightarrow$ (23)
(Figura 5.19.a)

Donde los trímeros (3) y (5) llegan al mismo pentámero, por lo que llegarán al mismo hexámero, siendo éste crecimiento el más probable para todos los agregados. En este patrón de crecimiento se obtiene un pentámero no plano, con un electrón desapareado, que se ubica en los átomos de fósforo, al igual que las zonas de ataque electrofílico.

\subsection{Conclusiones}

Se han obtenido las geometrías, energías de atomización, frecuencias de vibración armónicas, potenciales electrostáticos moleculares, cargas ESP, densidades de espín y patrones de crecimiento de clusters de Al y $\mathrm{P}$. En los trímeros y tetrámeros las energías de atomización encontradas son mayores que las reportadas en literatura, lo que indica que la metodología seguida para lograr estructuras estables es exitosa. 
El dímero AlP se estabiliza con un estado electrónico triplete y una energía de atomización de $1.13 \mathrm{eV} /$ át. El trímero más estable es AlPP (3), de estado electrónico doblete y estructura lineal, con una $\mathrm{E}_{\mathrm{at}}$ de $1.94 \mathrm{eV} /$ át. Con respecto a los agregados de cuatro átomos, el más estable es AlPPP (8), de capa cerrada, una energía de atomización de $2.47 \mathrm{eV} / a ́ t$ y geometría cíclica con tres átomos de fósforo y uno de aluminio en su estructura. De los pentámeros el más estable es PPAlPP (23), con cuatro átomos de fósforo y uno de aluminio en su estructura no plana, cuya $\mathrm{E}_{\mathrm{at}}$ es de $2.47 \mathrm{eV} /$ át y de estado electrónico doblete. Por lo anteriormente expuesto, se observa que los agregados binarios Al/P se estabilizan como agregados con $N-1$ átomos de fósforo en su estructura, donde $N$ es el número total de átomos.

En los agregados constituidos por $\mathrm{Al}$ y $\mathrm{P}$, se presentan movimientos de tipo wagging y scissoring en frecuencias bajas; en frecuencias intermedias se presentan estiramientos simétricos y asimétricos Al-Al y Al-P y a más de $400 \mathrm{~cm}^{-1}$, estiramientos $\mathrm{P}-\mathrm{P}$, en algunas ocasiones acoplados con estiramientos de otro tipo.

Los potenciales electrostáticos moleculares y cargas derivadas de ellos permiten explicar los patrones de crecimiento encontrados. La mayoría de los agregados crece hacia un pentámero no plano. El trímero AlAlP (2) no presenta patrón de crecimiento. Se informaron los patrones de crecimiento más probables de cada trímero, donde el crecimiento AlP (1) $\rightarrow$ AlPP (3) $\rightarrow$ PAlPP (9) $\rightarrow$ PPAlPP (23) es el más probable, donde el átomo atacante es siempre un átomo de fósforo y se une a una zona susceptible de ataque electrofílico. Asimismo, tanto el aluminio como el fósforo actuando como atacante se comportan como electrófilos en la mayoría de los casos. 


\section{Estudio de la estructura, modos vibracionales y patrones de crecimiento de clusters de boro y fósforo}

Los compuestos conformados por boro y fósforo constituyen sistemas de interés para la química y física de estado sólido debido a su potencialidad como semiconductores refractarios. Los pocos estudios experimentales encontrados hasta el momento se centran en obtener estructuras estables mediante deposición de vapor y caracterizarlas por métodos espectroscópicos ${ }^{90}$. Los estudios teóricos tampoco son numerosos y se enfocan en conocer las geometrías, estructuras electrónicas, estabilidades y modos vibracionales de clusters binarios. $\mathrm{Qu}$ y colaboradores ${ }^{91}$ estudiaron la estructura electrónica y estabilidad de agregados de (BP) ${ }_{n}$ donde $n=2-4$, usando el funcional híbrido de intercambio y correlación B3LYP y los conjuntos de funciones base $6-31 \mathrm{G}(\mathrm{d})$ y $6-311++\mathrm{G}(2 \mathrm{~d}, 2 \mathrm{p})$. Linguerri y coautores ${ }^{90}$ estudiaron los estados electrónicos de $\mathrm{BP}, \mathrm{BP}^{+}, \mathrm{BP}^{-}, \mathrm{B}_{2} \mathrm{P}_{2},\left(\mathrm{~B}_{2} \mathrm{P}_{2}\right)^{+}$y $\left(\mathrm{B}_{2} \mathrm{P}_{2}\right)^{-}$, utilizando CCSD y el conjunto de funciones base cc-pVQZ, para la obtención de estructuras estables y cálculos de frecuencias armónicas. Shi y colaboradores ${ }^{92}$ estudiaron la estructura, superficies de energía potencial y estabilidades de agregados planos de $\mathrm{B}_{\mathrm{n}} \mathrm{P}(n=1-7)$, a nivel $\operatorname{CCSD}(\mathrm{T}) / 6$ $311+\mathrm{G}(\mathrm{d}) / / \mathrm{B} 3 \mathrm{LYP} / 6-311+\mathrm{G}(\mathrm{d})$. Burril y Grein ${ }^{71}$ estudiaron las propiedades estructurales de clusters del tipo $\mathrm{X}_{2} \mathrm{Y}_{2}(\mathrm{X}=\mathrm{B}, \mathrm{Al}, \mathrm{Ga} ; \mathrm{Y}=\mathrm{N}, \mathrm{P}, \mathrm{As})$ usando DFT, el funcional B3LYP y el conjunto de funciones base $6-311+\mathrm{G}(3 \mathrm{df})$.

En el presente capítulo informamos los resultados obtenidos para agregados binarios de $\mathrm{B}_{\mathrm{n}} \mathrm{P}_{\mathrm{m}}$, con $n=1-4, m=1-4$ y $n+m \leq 5$. Se reportan propiedades estructurales, energías de atomización y frecuencias vibracionales para los agregados más estables.

El estudio de los patrones de crecimiento se realizó conformando los agregados más allá del dímero por la adición de átomos de boro o fósforo. El análisis de los patrones de crecimiento se llevó a cabo por medio de potenciales electrostáticos moleculares (MEP), densidades de espín y cargas derivadas de potenciales electrostáticos (cargas ESP).

\subsection{Estructuras, energías y modos vibracionales}

\section{Dímero BP}

El estado fundamental del dímero BP presenta estado electrónico triplete, con una energía de atomización $\left(E_{a t}\right)$ de $1.75 \mathrm{eV} /$ át (Tabla 6.1). La distancia de enlace de equilibrio es de $1.74 \AA$ (Figura 6.1). Estos datos están en acuerdo con Linguerri y colaboradores ${ }^{90}$, quienes informan al dímero con estado electrónico triplete y una distancia interatómica de $1.75 \AA$ A.

\section{Trímeros}

Se obtuvieron cuatro trímeros para el sistema $\mathrm{B} / \mathrm{P}$. Todos ellos presentan estado electrónico doblete (Tabla 6.1), siendo (4) el más estable con una $\mathrm{E}_{\mathrm{at}}$ de $2.81 \mathrm{eV} / a ́ t$, de geometría no lineal $\mathrm{y}$ con dos átomos de boro y uno de fósforo en su estructura (Figura 6.1). El aumento en la energía de atomización (Tabla 6.1), es el siguiente: $\mathbf{( 2 )}<\mathbf{( 3 )}<\mathbf{( 5 )}<\mathbf{( 4 )}$. Los modos normales de vibración se muestran en la Tabla 6.2. 
Tabla 6.1. Multiplicidades de espín $\left(\mathrm{M}_{\mathrm{S}}\right)$, energías de atomización $\left(\mathrm{E}_{\mathrm{at}}\right)$ en eV/át, del dímero y trímeros.

\begin{tabular}{c|c|c} 
Agregado & $\mathbf{M}_{\mathbf{S}}$ & $\mathbf{E}_{\text {at }}$ \\
\hline $\mathbf{( 1 )}$ & 3 & 1.75 \\
\hline & $3^{90,92}$ & \\
\hline $\mathbf{( 2 )}$ & 2 & 2.00 \\
\hline $\mathbf{( 3 )}$ & 2 & 2.58 \\
\hline $\mathbf{( 4 )}$ & 2 & 2.81 \\
\hline & $2^{92}$ & \\
\hline $\mathbf{( 5 )}$ & 2 & 2.64
\end{tabular}

(1)

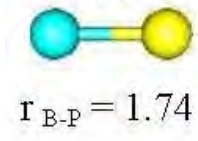

(2)

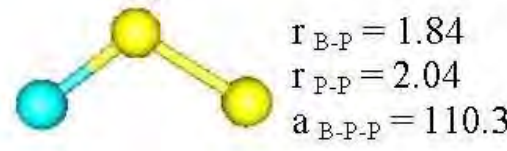

(3)

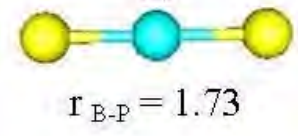

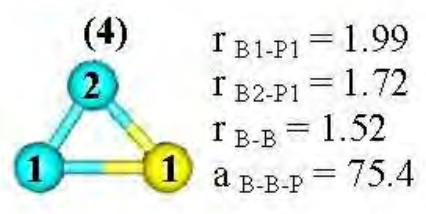

(5)

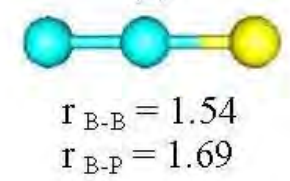

Figura 6.1. Geometrías de equilibrio para el dímero de partida y los trímeros. Las distancias (r) están expresadas en $\AA$ y los ángulos (a) en ${ }^{\circ}$. Los ángulos diedros son $0.0^{\circ}$ y los ángulos planos que no se especifican 180.0 , para todos los casos.

Por la Figura 6.1 se evidencia que dos trímeros presentan estructura de tipo lineal, (3) y (5), y dos no lineal, (2) y (4). Las uniones B-P se encuentran entre 1.69 y $1.99 \AA$, las B-B entre 1.52 y $1.54 \AA$ y la única unión P-P posee un valor de $2.04 \AA$.

Tabla 6.2. Modos normales de vibración $\left(\omega_{\mathrm{e}}\right)$ en $\mathrm{cm}^{-1}$, del dímero y los trímeros. ${ }^{*}$ significa que el modo es doblemente degenerado.

\begin{tabular}{c|c} 
Agregado & $\boldsymbol{\omega}_{\mathbf{e}}$ \\
\hline $\mathbf{( 1 )}$ & 956 \\
\hline & $941^{90}$ \\
\hline $\mathbf{( 2 )}$ & $130,454,672$ \\
\hline $\mathbf{( 3 )}$ & $46,189,510,1242$ \\
\hline $\mathbf{( 4 )}$ & $398,979,1176$ \\
\hline $\mathbf{( 5 )}$ & $128^{*}, 681,1399$
\end{tabular}

En la Tabla 6.2 se muestran los modos vibracionales del dímero y los trímeros. Se observa que el estiramiento B-P se encuentra a $956 \mathrm{~cm}^{-1}$, en acuerdo con lo reportado en otros trabajos. Por otro lado, en los trímeros, a frecuencias bajas se encuentran movimientos de tipo wagging $\mathrm{y}$ scissoring $\mathrm{y}$ a frecuencias intermedias $\mathrm{y}$ altas, estiramientos simétricos $\mathrm{y}$ asimétricos.

\section{Tetrámeros}

Se calcularon los agregados binarios de cuatro átomos, partiendo de los trímeros antes descriptos, obteniéndose 11 clusters. El más estable de los tetrámeros es el agregado (11), de 
estado electrónico triplete y $\mathrm{E}_{\text {at }}$ de $3.24 \mathrm{eV} /$ át (Tabla 6.3). Este agregado posee tres átomos de boro y uno de fósforo en su estructura, la cual es plana y cíclica (Figura 6.2).

Tabla 6.3. Multiplicidades de espín $\left(\mathrm{M}_{\mathrm{S}}\right)$ y energías de atomización $\left(\mathrm{E}_{\mathrm{at}}\right)$ en eV/át, de los tetrámeros.

\begin{tabular}{c|c|c} 
Agregado & $\mathbf{M}_{\mathbf{S}}$ & $\mathbf{E}_{\mathbf{a t}}$ \\
\hline $\mathbf{( 6 )}$ & 3 & 2.87 \\
\hline & $3^{71,90,91}$ & \\
\hline $\mathbf{( 7 )}$ & 1 & 3.05 \\
\hline $\mathbf{( 8 )}$ & 3 & 3.03 \\
\hline $\mathbf{( 9 )}$ & 1 & 2.69 \\
\hline $\mathbf{( 1 0 )}$ & 1 & 2.34 \\
\hline $\mathbf{( 1 1 )}$ & 3 & 3.24 \\
\hline & $1^{92}$ & \\
\hline $\mathbf{( 1 2 )}$ & 3 & 3.11 \\
\hline $\mathbf{( 1 3 )}$ & 3 & 2.89 \\
\hline $\mathbf{( 1 4 )}$ & 3 & 2.62 \\
\hline $\mathbf{( 1 5 )}$ & 3 & 2.48 \\
\hline $\mathbf{( 1 6 )}$ & 3 & 2.90
\end{tabular}

Se observa en la Tabla 6.3 que la energía de atomización aumenta en el siguiente orden: (10) $<\mathbf{( 1 5 )}<\mathbf{( 1 4 )}<\mathbf{( 9 )}<\mathbf{( 6 )}<\mathbf{( 1 3 )}<\mathbf{( 1 6 )}<\mathbf{( 8 )}<\mathbf{( 7 )}<\mathbf{( 1 2 )}<\mathbf{( 1 1 )}$. Se observa también una preferencia a estabilizarse con dos electrones desapareados. Asimismo, de los agregados de cuatro átomos sólo tres son lineales, (9), (12) y (13); y dos no planos, (6) y 14) (Figura 6.2). 
(6)

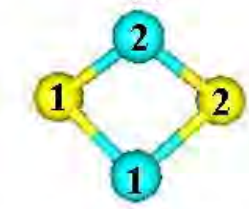

$\mathrm{r}_{\mathrm{B}-\mathrm{P}}=1.86$

$\mathrm{a}_{\mathrm{B} 1-\mathrm{P} 1-\mathrm{B} 2}=70.0$

$\mathrm{a}_{\mathrm{P} 1-\mathrm{B} 2 \mathrm{P} 2}=87.8$

$\mathrm{d}_{\mathrm{B} 1-\mathrm{P} 1-\mathrm{B} 2-\mathrm{P} 2}=47.6$

(10)
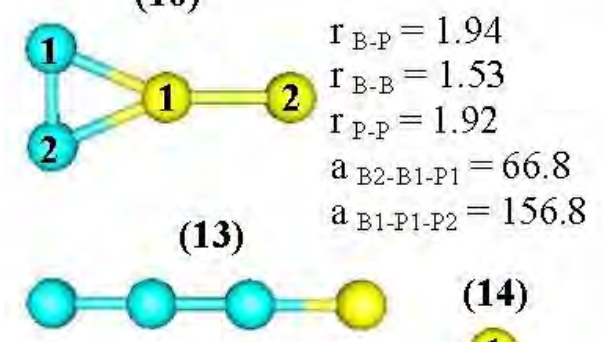

(14)

$\mathrm{r}_{\mathrm{B} 1-\mathrm{B} 2}=1.56$

$\mathrm{r}_{\mathrm{B} 2-\mathrm{B} 3}=1.53$

$\mathrm{r}_{\mathrm{P}-\mathrm{B}}=1.71$
(7)

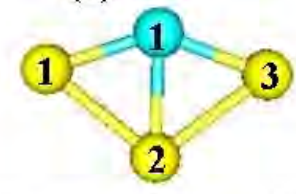

$\mathrm{r}_{\mathrm{B} 1-\mathrm{P} 1}=\mathrm{r}_{\mathrm{B1}-\mathrm{P} 3}=1.81$

$\mathrm{r}_{\mathrm{P}-\mathrm{P}}=2.17$

$\mathrm{r}_{\mathrm{B} 1-\mathrm{P} 2}=1.94$

$\mathrm{a}_{\mathrm{P} 2 \mathrm{-P} 1-\mathrm{B} 1}=57.5$

$a_{P 1-B 1-P 3}=140.9$
(8)

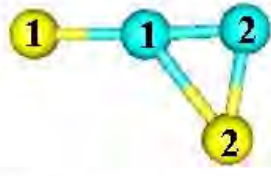

(9)

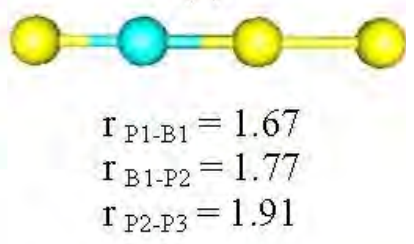

(12)

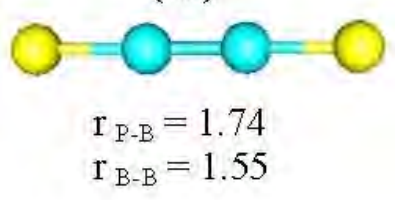

(11)

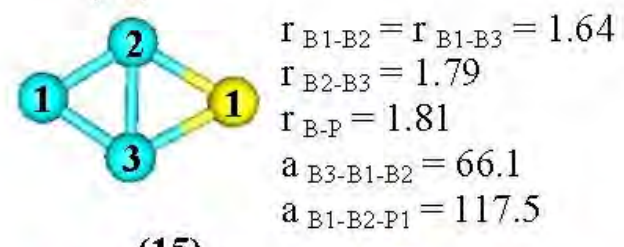

1 (15)
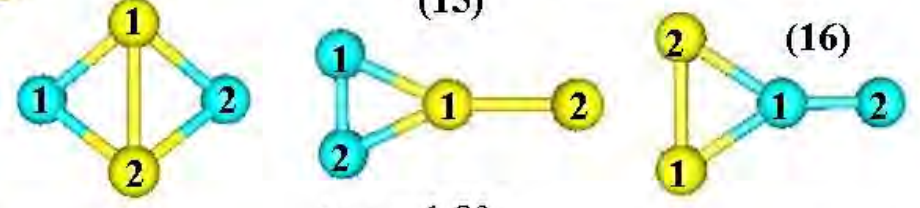

$\mathrm{r}_{\text {B-P }}=1.92$
$\mathrm{r}_{\text {P-P }}=2.28$
$\mathrm{a}_{\text {B1-P1-B2 }}=98.3$
$\mathrm{a}_{\text {P1-B2-P2 }}=72.6$
$\mathrm{~d}_{\text {B1-P1-B2-P2 }}=31.7$

$\mathrm{r}_{\mathrm{B}-\mathrm{P}}=1.89$

$\mathrm{r}_{\mathrm{B}-\mathrm{B}}=1.57$

$\mathrm{r}_{\mathrm{P}-\mathrm{P}}=1.99$

$\mathrm{r}_{\mathrm{B}-\mathrm{P}}=1.88$

$\mathrm{r}_{\mathrm{B}-\mathrm{B}}=1.55$

$\mathrm{a}_{\mathrm{B} 2-\mathrm{B} 1-\mathrm{P} 1}=65.3$

$\mathrm{a}_{\mathrm{B} 1-\mathrm{P} 1-\mathrm{P} 2}=155.4$

$\mathrm{r}_{\mathrm{P}-\mathrm{P}}=2.05$

$\mathrm{a}_{\mathrm{P} 1-\mathrm{P} 2-\mathrm{B} 1}=56.9$

$a_{P 2-B 1-B 2}=146.9$

Figura 6.2. Geometrías de equilibrio de los tetrámeros. Las distancias (r) están expresadas en $\AA$, y los ángulos planos (a) y diedros (d), en ${ }^{\circ}$.

A partir de la Figura 6.2 se observa que las distancias interatómicas B-B toman valores entre 1.53 y $1.64 \AA$; las distancias B-P entre 1.67 y $1.94 \AA$ y las distancias P-P entre 1.92 y $2.07 \AA$, siendo mayores las distancias en los agregados no lineales. Lo reportado para el tetrámero romboidal (6) se encuentra en concordancia con los parámetros geométricos reportados por Burril y Grein ${ }^{101}$, Linguerri y coautores ${ }^{90}$, y Qu y colaboradores ${ }^{91}$, quienes reportan una distancia interatómica B-P de $1.84 \AA$ A . Estos autores reportan al rombo alternado como agregado más estable. Asimismo, para $\mathrm{Qu}$ y colaboradores ${ }^{91}$ el isómero de (11) más estable es el de estado electrónico singlete, a nivel B3LYP/6-311+G(d). 
Tabla 6.4. Frecuencias vibracionales $\left(\omega_{\mathrm{e}}\right)$ de los tetrámeros en $\mathrm{cm}^{-1} . *$ significa que el modo es doblemente degenerado.

\begin{tabular}{c|c} 
Agregado & $\boldsymbol{\omega}_{\mathbf{e}}$ \\
\hline $\mathbf{( 6 )}$ & $288,398,474,739,795,801$ \\
\hline & $258,402,442,455,526,709^{91}$ \\
\hline $\mathbf{( 7 )}$ & $211,302,386,493,724,938$ \\
\hline $\mathbf{( 8 )}$ & $122,273,345,590,993,1153$ \\
\hline $\mathbf{( 9 )}$ & $74^{*}, 204^{*}, 396,811,1308$ \\
\hline $\mathbf{( 1 0 )}$ & $147,170,250,504,784,1123$ \\
\hline $\mathbf{( 1 )}$ & $197,500,601,857,865,1109$ \\
\hline $\mathbf{( 1 2 )}$ & $95^{*}, 335^{*}, 404,878,1465$ \\
\hline $\mathbf{( 1 3 )}$ & $83,114,301,364,537,1064,1543$ \\
\hline $\mathbf{( 1 4 )}$ & $286,409,444,462,529,712$ \\
\hline $\mathbf{( 1 5 )}$ & $122,134,369,503,666,1054$ \\
\hline $\mathbf{( 1 6 )}$ & $121,299,510,554,653,1229$
\end{tabular}

En la Tabla 6.4 se listan los modos vibracionales de los tetrámeros. Del análisis se desprende que a frecuencias bajas los agregados de cuatro átomos presentan movimientos de tipo wagging, twisting y scissoring; a frecuencias intermedias, estiramientos B-P y P-P simétricos y asimétricos; y a frecuencias mayores a 1109 se encuentran estiramientos B-B simétricos y asimétricos. Qu y colaboradores ${ }^{91}$ informan frecuencias vibracionales para el cluster romboidal alternado (6), similares a las encontradas en este trabajo.

\section{Pentámeros}

Se obtuvieron 28 pentámeros B/P, de los cuales sólo dos son lineales. Para formar estos agregados se adicionaron átomos de boro y fósforo en todos los sitios posibles de los tetrámeros descriptos anteriormente. Se presentan los resultados obtenidos en las Figuras 6.3 a 6.6 y Tablas 6.5 y 6.6 . 
(17)

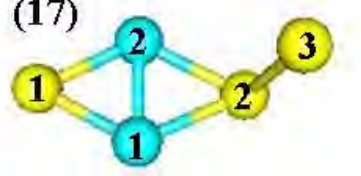

$\mathrm{r}_{\mathrm{P} 1-\mathrm{B} 2}=\mathrm{r}_{\mathrm{P} 1-\mathrm{B} 1}=1.80$

$\mathrm{r}_{\mathrm{B} 1-\mathrm{P} 2}=\mathrm{r}_{\mathrm{B} 2-\mathrm{P} 2}=1.91$

$\mathrm{r}_{\mathrm{B}-\mathrm{B}}=1.71$

$\mathrm{r}_{\mathrm{P}-\mathrm{P}}=1.19$

$\mathrm{a}_{\mathrm{B} 1-\mathrm{P} 1-\mathrm{B} 2}=56.5$

$\mathrm{a}_{\mathrm{P} 1-\mathrm{B} 2-\mathrm{P} 2}=125.1$

$\mathrm{d}_{\mathrm{P} 1-\mathrm{B} 1-\mathrm{P} 2-\mathrm{P} 3}=99.7$

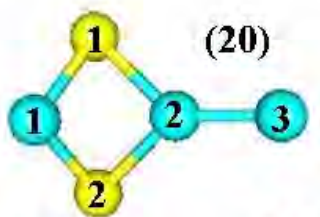

$\mathrm{r}_{\mathrm{P} 1-\mathrm{B} 1}=\mathrm{r}_{\mathrm{P} 2-\mathrm{B} 1}=1.85$

$\mathrm{r}_{\mathrm{B} 2-\mathrm{P} 1}=\mathrm{r}_{\mathrm{B} 2-\mathrm{P} 2}=1.89$

$\mathrm{r}_{\mathrm{B}-\mathrm{B}}=1.66$

$\mathrm{a}_{\mathrm{P} 1-\mathrm{B} 1-\mathrm{P} 2}=86.1$

$a_{P 1-B 2-B 3}=137.3$

$\mathrm{d}_{\mathrm{B} 1-\mathrm{P} 2-\mathrm{B} 2-\mathrm{B} 3}=143.7$

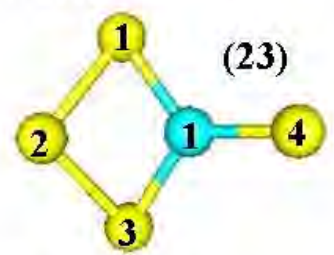

$\mathrm{r}_{\mathrm{P}-\mathrm{P}}=2.14$

$\mathrm{r}_{\mathrm{P} 1-\mathrm{B} 1}=\mathrm{r}_{\mathrm{P} 3-\mathrm{B} 1}=1.91$

$\mathrm{r}_{\mathrm{B} 1-\mathrm{P} 4}=1.90$

$\mathrm{a}_{\mathrm{P} 1-\mathrm{B} 1 \mathrm{P} 3}=106.5$

$a_{\mathrm{P} 3-\mathrm{B} 1-\mathrm{P} 4}=126.5$

$\mathrm{d}_{\text {P2-P3-B1-P4 }}=130.6$
(18)

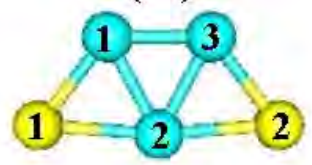

$\mathrm{r}_{\mathrm{P} 1-\mathrm{B} 2}=\mathrm{r}_{\mathrm{P} 2-\mathrm{B} 2}=1.89$

$\mathrm{r}_{\mathrm{B} 1-\mathrm{P} 1}=\mathrm{r}_{\mathrm{B} 3-\mathrm{P} 2}=1.76$

$\mathrm{r}_{\mathrm{B} 1-\mathrm{B} 3}=1.60$

$\mathrm{r}_{\mathrm{B} 1-\mathrm{B} 2}=\mathrm{r}_{\mathrm{B} 3-\mathrm{B} 2}=1.73$

$\mathrm{a}_{\mathrm{P} 1-\mathrm{B} 2-\mathrm{P} 2}=171.2$

$\mathrm{a}_{\mathrm{P} 1-\mathrm{B} 1-\mathrm{B} 3}=127.9$

(21)

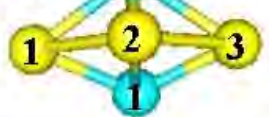

$\mathrm{r}_{\mathrm{P} 1-\mathrm{B} 2}=\mathrm{r}_{\mathrm{B} 2-\mathrm{P} 3}=1.96$

$r_{B 1-P 1}=r_{B 1-P 3}=1.80$

$\mathrm{r}_{\mathrm{B} 2-\mathrm{P} 2}=1.92$

$r_{P 2-B 1}=2.26$

$a_{P 1-B 2-P 3}=109.6$

$\mathrm{a}_{\mathrm{B} 1-\mathrm{P} 1-\mathrm{B} 2}=61.0$

$\mathrm{d}_{\mathrm{B} 1-\mathrm{P} 3-\mathrm{B} 2-\mathrm{P} 2}=79.0$

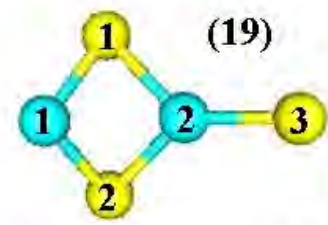

$\mathrm{r}_{\mathrm{P} 1-\mathrm{B} 2}=\mathrm{r}_{\mathrm{P} 2-\mathrm{B} 2}=1.91$

$\mathrm{r}_{\mathrm{B} 1-\mathrm{P} 1}=\mathrm{r}_{\mathrm{B} 1-\mathrm{P} 2}=\mathrm{r}_{\mathrm{B} 2-\mathrm{P} 3}=1.83$

$\mathrm{a}_{\mathrm{P} 1-\mathrm{B} 1-\mathrm{P} 2}=89.7$

$a_{P 1-B 2-P 3}=136.6$

$d_{B 1-P 2-B 2-P 3}=146.1$

(2) $1 \mathrm{r}_{\mathrm{P} 1-\mathrm{B} 1}=\mathrm{r}_{\mathrm{P} 2-\mathrm{B} 1}=1.85$

(22)

$\mathrm{r}_{\mathrm{B} 1-\mathrm{P} 3}=1.88$

$\mathrm{r}_{\mathrm{P}-\mathrm{P}}=2.01$

$\mathrm{a}_{\mathrm{P} 1-\mathrm{B} 1-\mathrm{P} 3}=145.7$

$\mathrm{a}_{\mathrm{B} 1-\mathrm{P} 3-\mathrm{P} 4}=101.2$

$\mathrm{d}_{\mathrm{P} 1-\mathrm{B} 1-\mathrm{P} 3-\mathrm{P} 4}=94.9$

(4)

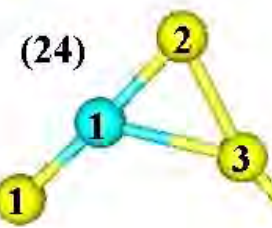

$\mathrm{r}_{\mathrm{P} 1-\mathrm{B} 1}=1.74$

$\mathrm{r}_{\mathrm{P} 2 \mathrm{~B} 1}=1.83$

$r_{\mathrm{P} 2-\mathrm{P} 3}=2.09$

$\mathrm{r}_{\mathrm{P} 3-\mathrm{P} 4}=1.92$

$\mathrm{r}_{\mathrm{B} 1-\mathrm{P} 3}=2.27$

$\mathrm{a}_{\mathrm{P} 1-\mathrm{B} 1-\mathrm{P} 2}=177.4$

$\mathrm{a}_{\mathrm{B} 1-\mathrm{P} 2 \mathrm{P} 3}=70.3$

Figura 6.3. Geometrías de equilibrio de los pentámeros (17) a (24). Las distancias (r) están expresadas en $\AA$, los ángulos planos (a) y los diedros (d), en ${ }^{\circ}$. Los ángulos planos que no se especifican valen $180^{\circ}$ y los diedros $0.0^{\circ}$. 


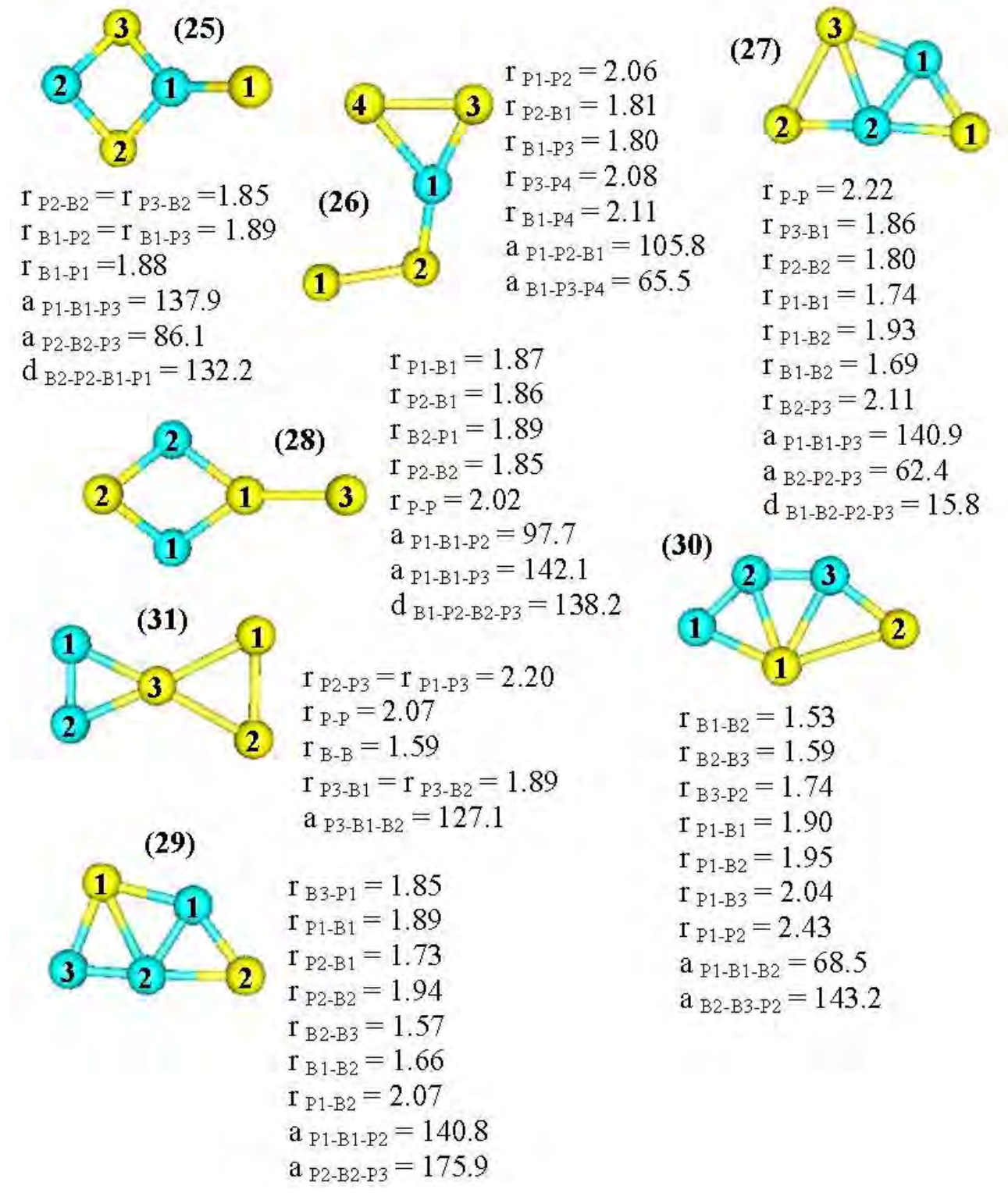

Figura 6.4. Geometrías de equilibrio de los pentámeros (25) a (31). Las distancias (r) están expresadas en $\AA$, los ángulos planos (a) y los diedros (d), en ${ }^{\circ}$. Los ángulos planos que no se especifican valen $180^{\circ}$ y los diedros $0.0^{\circ}$. 


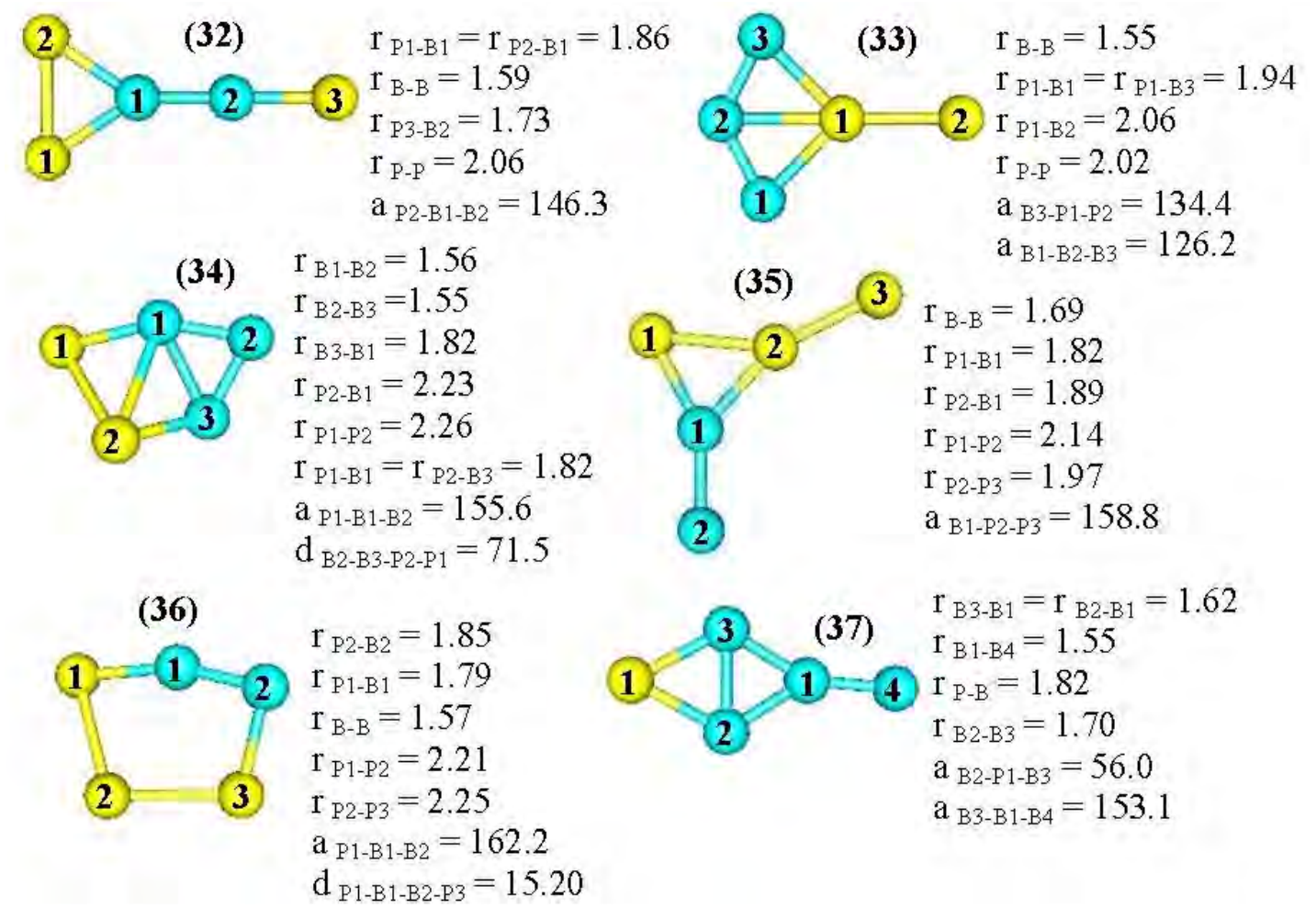

Figura 6.5. Geometrías de equilibrio de los pentámeros (32) a (37). Las distancias (r) están expresadas en $\AA$, los ángulos planos (a) y los diedros (d), en ${ }^{\circ}$. Los ángulos planos que no se especifican valen $180^{\circ}$ y los diedros $0.0^{\circ}$. 

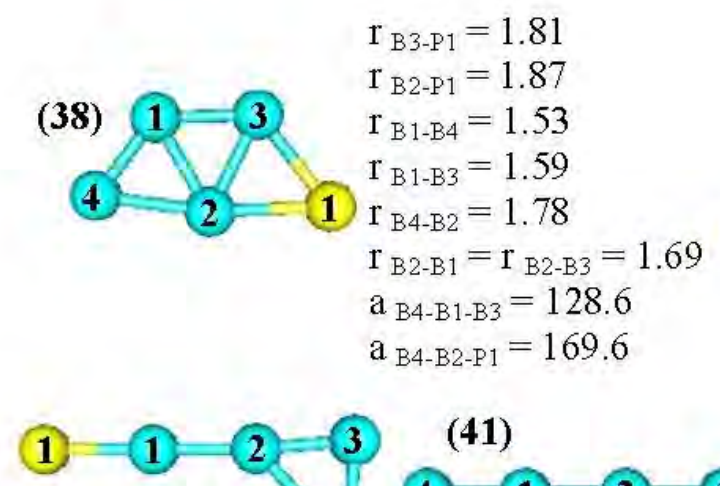

(40)

$$
\begin{aligned}
& r_{P 1-B 1}=1.73 \\
& r_{B 1-B 2}=1.60 \\
& r_{B 2-B 3}=1.52 \\
& r_{B 3-P 2}=1.70 \\
& r_{B 2-P 2}=2.11 \\
& a_{B 2-B 3-P 2}=81.7
\end{aligned}
$$

\section{$(4-1)$}

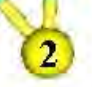
$\mathrm{r}_{\mathrm{B} 4-\mathrm{B} 1}=1.53$
$\mathrm{r}_{\mathrm{B} 1-\mathrm{B} 2}=1.55$
$\mathrm{r}_{\mathrm{B} 2-\mathrm{B} 3}=1.54$
$\mathrm{r}_{\mathrm{B} 3-\mathrm{P} 1}=1.72$

$\mathrm{r}_{\mathrm{B} 4-\mathrm{P} 1}=1.81$

$\mathrm{r}_{\mathrm{B} 2-\mathrm{P} 1}=1.88$

$\mathrm{r}_{\mathrm{B} 1-\mathrm{B} 3}=1.51$

$\mathrm{r}_{\mathrm{B} 3-\mathrm{B} 4}=1.56$

$r_{B 1-B 2}=1.54$

$r_{B 2-B 3}=1.87$

$\mathrm{r}_{\mathrm{B} 2-\mathrm{B} 4}=1.75$

$\mathrm{a}_{B 4-B 3-B 1}=113.5$

$\mathrm{a}_{\mathrm{B} 1-\mathrm{B} 2-\mathrm{P} 1}=162.3$

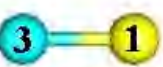

(42)

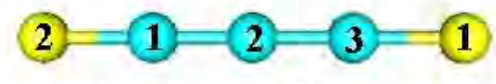

$\mathrm{r}_{\mathrm{B}-\mathrm{B}}=1.56$

$\mathrm{r}_{\mathrm{P}-\mathrm{B}}=1.71$

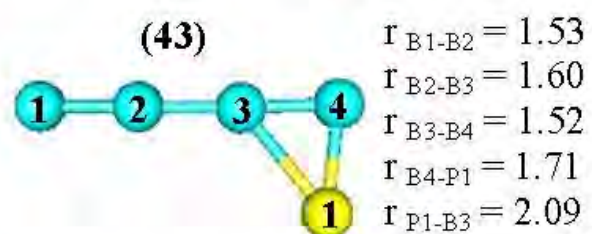

(1) $\mathrm{r}_{\mathrm{P} 1-\mathrm{B} 3}=2.09$

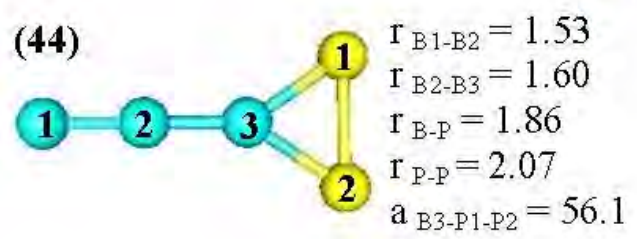

$a_{B 3-B 4-P 1}=80.5$

Figura 6.6. Geometrías de equilibrio de los pentámeros (38) a (44). Las distancias (r) están expresadas en $\AA$, los ángulos planos (a) y los diedros (d), en ${ }^{\circ}$. Los ángulos planos que no se especifican valen $180^{\circ}$ y los diedros $0.0^{\circ}$.

De las Figuras 6.3 a 6.6 se desprende que en los pentámeros las distancias de unión P-P toman valores entre 1.96 y $2.22 \AA$; las uniones P-B entre 1.76 y $1.96 \AA$; y las distancias B-B entre 1.51 y $1.82 \AA ̊$. 
Tabla 6.5. Multiplicidades de espín $\left(\mathrm{M}_{\mathrm{S}}\right)$ y energías de atomización $\left(\mathrm{E}_{\mathrm{at}}\right)$, en eV/át, de los pentámeros.

\begin{tabular}{|c|c|c|}
\hline \\
\hline Agregado & $\mathbf{M}_{\mathbf{S}}$ & $\mathbf{E}_{\mathbf{a t}}$ \\
\hline (17) & 4 & 3.00 \\
\hline (18) & 2 & 3.70 \\
\hline (19) & 2 & 3.01 \\
\hline (20) & 2 & 2.98 \\
\hline (21) & 2 & 3.26 \\
\hline$(22)$ & 2 & 2.97 \\
\hline (23) & 4 & 2.79 \\
\hline$(24)$ & 2 & 2.67 \\
\hline (25) & 4 & 2.96 \\
\hline (26) & 4 & 2.67 \\
\hline (27) & 2 & 3.35 \\
\hline (28) & 4 & 2.60 \\
\hline (29) & 2 & 3.45 \\
\hline (30) & 2 & 3.31 \\
\hline (31) & 2 & 2.55 \\
\hline (32) & 2 & 3.32 \\
\hline (33) & 4 & 2.72 \\
\hline (34) & 2 & 3.32 \\
\hline (35) & 2 & 2.62 \\
\hline (36) & 4 & 2.92 \\
\hline (37) & 4 & 3.40 \\
\hline (38) & 4 & 3.50 \\
\hline (39) & 2 & 3.73 \\
\hline & $2^{92}$ & \\
\hline (40) & 2 & 3.37 \\
\hline (41) & 4 & 3.25 \\
\hline$(42)$ & 2 & 3.35 \\
\hline (43) & 4 & 3.25 \\
\hline (44) & 4 & 3.17 \\
\hline
\end{tabular}

Se observa que el pentámero más estable, el agregado (39) (Tabla 6.5), presenta estado electrónico doblete y una energía de atomización de $3.73 \mathrm{eV} /$ át. Con cuatro átomos de boro y uno de fósforo, presenta una estructura plana (Figura 6.4) y gran cantidad de uniones interatómicas. Asimismo, la energía de atomización aumenta en el siguiente orden: (31) < (28) $<$ (35) $<$ (26) $<$ (24) $<$ (33) $<$ (23) $<$ (36) $<$ (25) $<$ (22) $<$ (20) $<$ (17) $<$ (19) $<$ (44) $<$ (41) $<$ (43) $<$ (21) $<$ (30) < (34) < (32) < (42) < (27) < (40) < (37) < (29) < (38) < (18) < (39). Es importante mencionar que las energías de atomización, expresadas con tres decimales, de los agregados (26), (24), (41), (43), (34) y (32) son, respectivamente: $2.666 \mathrm{eV} / a ́ t, 2.668 \mathrm{eV} / a ́ t$, $3.253 \mathrm{eV} / a ́ t, 3.255 \mathrm{eV} / a ́ t, 3.319 \mathrm{eV} /$ át y $3.321 \mathrm{eV} / a ́ t$.

Qu y colaboradores ${ }^{92}$ también encuentran al isómero (39) como más estable.

En la Tabla 6.6 se listan los modos normales de vibración correspondientes a los pentámeros. 
Tabla 6.6. Modos normales de vibración $\left(\omega_{\mathrm{e}}\right)$ de los pentámeros, en $\mathrm{cm}^{-1}$.

\begin{tabular}{c|c} 
Agregado & $\boldsymbol{\omega}_{\mathbf{e}}$ \\
\hline $\mathbf{( 1 7 )}$ & $81,209,310,353,419,474,747,843,980$ \\
\hline $\mathbf{( 1 8 )}$ & $273,286,331,539,560,685,836,1006,1196$ \\
\hline $\mathbf{( 1 9 )}$ & $141,174,237,336,433,472,735,804,928$ \\
\hline $\mathbf{( 2 0 )}$ & $107,132,283,328,478,518,764,788,975$ \\
\hline $\mathbf{( 2 1 )}$ & $208,302,326,429,480,602,650,755,1009$ \\
\hline $\mathbf{( 2 )}$ & $49,72,157,308,372,573,617,647,1010$ \\
\hline $\mathbf{( 2 3 )}$ & $126,134,251,331,371,389,521,646,817$ \\
\hline $\mathbf{( 2 4 )}$ & $49,110,135,232,234,395,471,787,1109$ \\
\hline $\mathbf{( 2 5 )}$ & $147,171,269,337,417,488,759,793,927$ \\
\hline $\mathbf{( 2 )}$ & $43,76,178,233,295,350,485,542,979$ \\
\hline $\mathbf{( 2 7 )}$ & $168,255,313,332,408,469,723,938,1084$ \\
\hline $\mathbf{( 2 8 )}$ & $34,102,252,312,394,527,655,762,806$ \\
\hline $\mathbf{( 2 9})$ & $187,279,329,390,459,661,861,996,1174$ \\
\hline $\mathbf{( 3 0 )}$ & $116,182,345,397,532,547,749,1110,1339$ \\
\hline $\mathbf{( 3 1 )}$ & $75,123,146,293,372,403,593,699,1022$ \\
\hline $\mathbf{( 3 2})$ & $72,120,324,328,412,584,614,914,1344$ \\
\hline $\mathbf{( 3 3 )}$ & $123,145,166,417,483,564,667,991,1256$ \\
\hline $\mathbf{( 3 4 )}$ & $223,271,336,372,500,620,779,1088,1132$ \\
\hline $\mathbf{( 3 5 )}$ & $92,120,146,215,376,456,607,687,1081$ \\
\hline $\mathbf{( 3 6 )}$ & $113,195,250,294,360,406,639,730,1189$ \\
\hline $\mathbf{( 3 7 )}$ & $38,130,404,453,530,837,861,931,1362$ \\
\hline $\mathbf{( 3 8 )}$ & $151,341,362,452,581,781,903,995,1288$ \\
\hline $\mathbf{( 3 9})$ & $274,285,366,495,606,816,1070,1160,1315$ \\
\hline $\mathbf{( 4 0 )}$ & $83,142,256,412,434,515,1044,1111,1431$ \\
\hline $\mathbf{( 4 1 )}$ & $71,200,201,414,425,431,863,1305,1570$ \\
\hline $\mathbf{( 4 2 )}$ & $63,176,184,345,404,460,762,1262,1524$ \\
\hline $\mathbf{( 4 3 )}$ & $103,162,274,420,439,624,1025,1213,1447$ \\
\hline $\mathbf{( 4 4 )}$ & $102,144,363,404,415,593,630,1031,1398$
\end{tabular}

Del análisis se desprende que a frecuencias bajas los pentámeros presentan movimientos de tipo wagging, rocking, twisting y scissoring; a frecuencias intermedias, estiramientos B-P y P$\mathrm{P}$ simétricos y asimétricos; y a frecuencias mayores a 1006 se encuentran estiramientos B-B simétricos y asimétricos.

Análisis de cargas derivadas del potencial electrostático y de la densidad de espín

Los análisis de cargas derivadas de potenciales electrostáticos (ESP) y de la densidad de espín, se realizaron con el mismo nivel de teoría utilizado para obtener las geometrías de equilibrio y los modos normales de vibración. El cálculo de la densidad de espín se realizó mediante la obtención de la configuración electrónica natural. En las Tablas 6.7 a 6.9 y Figuras 6.7 a 6.8 se muestran los resultados obtenidos. 
Tabla 6.7. Análisis de cargas ESP (en unidades de |e|) y densidades de espín, entre paréntesis y en u.a., del dímero y los trímeros. Los átomos se numeran de izquierda a derecha o como se muestra en la Figura 6.1.

\begin{tabular}{c|c|c|c|c} 
Agregado & B1 & B2 & P1 & P2 \\
\hline $\mathbf{( 1 )}$ & $0.04(1.16)$ & & $-0.04(0.84)$ & \\
\hline $\mathbf{( 2 )}$ & $-0.09(0.27)$ & & $0.06(0.01)$ & $0.03(0.73)$ \\
\hline $\mathbf{( 3 )}$ & $-0.06(-0.16)$ & & $0.03(0.59)$ & $0.03(0.59)$ \\
\hline $\mathbf{( 4 )}$ & $-0.08(0.92)$ & $0.04(-0.03)$ & $0.04(0.11)$ & \\
\hline $\mathbf{( 5 )}$ & $0.20(1.27)$ & $-0.20(0.36)$ & $-0.007(-0.66)$ &
\end{tabular}


Tabla 6.8. Análisis de cargas ESP (en unidades de |e|) y densidades de espín, entre paréntesis y en u.a., de los tetrámeros. Los átomos se numeran de izquierda a derecha, o como se muestra en la Figura 6.2.

\begin{tabular}{c|c|c|c|c|c|c} 
Agregado & $\mathrm{B} 1$ & $\mathrm{~B} 2$ & $\mathrm{~B} 3$ & $\mathrm{P} 1$ & $\mathrm{P} 2$ & $\mathrm{P} 3$ \\
\hline $\mathbf{( 6 )}$ & $-0.04(1.03)$ & $-0.04(1.03)$ & & $0.04(-0.03)$ & $0.04(-0.03)$ & \\
\hline $\mathbf{( 7 )}$ & 0.09 & & & -0.10 & 0.12 & -0.10 \\
\hline $\mathbf{( 8 )}$ & $-0.02(-0.11)$ & $-0.006(0.19)$ & & $-0.0003(1.53)$ & $0.03(0.11)$ & \\
\hline $\mathbf{( 9 )}$ & -0.08 & & & 0.07 & 0.08 & -0.06 \\
\hline $\mathbf{( 1 0 )}$ & -0.08 & -0.09 & & 0.31 & -0.14 & \\
\hline $\mathbf{( 1 1 )}$ & $-0.06(0.87)$ & $0.001(0.54)$ & $0.002(0.54)$ & $0.05(-0.01)$ & & \\
\hline $\mathbf{( 1 2 )}$ & $0.002(-0.11)$ & $0.002(-0.11)$ & & $-0.002(1.11)$ & $-0.002(1.11)$ & \\
\hline $\mathbf{( 1 3 )}$ & $-0.08(1.59)$ & $0.02(0.84)$ & $-0.07(-0.22)$ & $-0.04(-0.22)$ & & \\
\hline $\mathbf{( 1 4 )}$ & $-0.19(0.55)$ & $-0.19(0.55)$ & & $0.19(0.45)$ & $0.19(0.45)$ & \\
\hline $\mathbf{( 1 5 )}$ & $-0.008(0.84)$ & $-0.004(0.84)$ & & $0.01(-0.12)$ & $-0.0003(0.41)$ & \\
\hline $\mathbf{( 1 6 )}$ & $0.007(0.39)$ & $-0.03(1.53)$ & & $0.01(0.06)$ & $0.01(0.06)$ &
\end{tabular}


Tabla 6.9. Análisis de cargas ESP (en unidades de |e|) y densidades de espín, entre paréntesis y en u.a., de los pentámeros. Los átomos se

\begin{tabular}{|c|c|c|c|c|c|c|c|c|}
\hline$\#$ & $\mathrm{~B} 1$ & B2 & B3 & B4 & P1 & P2 & $\mathrm{P} 3$ & $\mathrm{P} 4$ \\
\hline (17) & $0.04(0.65)$ & $0.04(0.65)$ & & & $-0.06(-0.08)$ & $-0.03(0.13)$ & $0.007(1.91)$ & \\
\hline (18) & $-0.03(0.06)$ & $0.07(-0.06)$ & $-0.03(0.04)$ & & $-0.01(0.50)$ & $-0.004(0.50)$ & & \\
\hline (19) & $0.04(0.10)$ & $0.12(-0.04)$ & & & $0.01(0.05)$ & $0.01(0.05)$ & $-0.19(0.84)$ & \\
\hline (20) & $-0.09(0.80)$ & $0.49(0.15)$ & $-0.32(0.09)$ & & $-0.04(0.01)$ & $-0.04(0.01)$ & & \\
\hline (21) & $-0.03(0.21)$ & $-0.07(0.79)$ & & & $0.05(-0.05)$ & $-0.001(0.08)$ & $0.05(-0.05)$ & \\
\hline (22) & $0.23(0.06)$ & & & & $-0.02(0.00)$ & $-0.13(0.04)$ & $-0.02(0.00)$ & $-0.05(0.93)$ \\
\hline (23) & $0.19(0.06)$ & & & & $-0.05(0.69)$ & $0.05(-0.10)$ & $-0.05(0.69)$ & $-0.14(1.76)$ \\
\hline (24) & $0.04(0.00)$ & & & & $-0.10(0.83)$ & $0.08(0.22)$ & $-0.04(0.00)$ & $0.02(0.04)$ \\
\hline (25) & $0.24(0.15)$ & $-0.04(0.94)$ & & & $-0.13(1.76)$ & $-0.03(0.10)$ & $-0.03(0.10)$ & \\
\hline (26) & $0.004(1.40)$ & & & & $-0.03(1.40)$ & $-0.05(0.09)$ & $0.06(0.01)$ & $0.01(1.04)$ \\
\hline (27) & $0.03(0.10)$ & $0.08(0.13)$ & & & $-0.01(0.41)$ & $-0.03(0.37)$ & $-0.08(0.00)$ & \\
\hline (28) & $-0.09(1.13)$ & $-0.09(1.11)$ & & & $0.17(-0.09)$ & $0.02(-0.15)$ & $-0.02(0.99)$ & \\
\hline (29) & $0.11(0.06)$ & $0.04(0.02)$ & $-0.04(0.93)$ & & $-0.0001(-0.02)$ & $-0.11(0.01)$ & & \\
\hline (30) & $-0.04(0.94)$ & $-0.04(0.01)$ & $0.07(0.05)$ & & $0.10(-0.03)$ & $-0.08(0.02)$ & & \\
\hline (31) & $-0.11(0.56)$ & $-0.11(0.56)$ & & & $0.12(-0.01)$ & $0.05(-0.06)$ & $0.05(-0.06)$ & \\
\hline (32) & $0.22(-0.01)$ & $-0.16(0.16)$ & & & $0.001(0.04)$ & $-0.06(0.78)$ & $0.002(0.04)$ & \\
\hline (33) & $-0.03(1.09)$ & $0.03(-0.03)$ & $-0.03(1.09)$ & & $0.11(-0.12)$ & $-0.08(0.95)$ & & \\
\hline (34) & $0.04(-0.16)$ & $-0.14(0.54)$ & $0.08(0.05)$ & & $0.01(0.67)$ & $0.01(-0.08)$ & & \\
\hline (35) & $0.54(0.08)$ & $-0.32(0.14)$ & & & $-0.09(0.16)$ & $-0.12(-0.11)$ & $-0.01(0.72)$ & \\
\hline (36) & $0.11(0.14)$ & $-0.11(1.15)$ & & & $-0.02(0.43)$ & $-0.01(0.85)$ & $0.03(0.44)$ & \\
\hline (37) & $-0.08(0.41)$ & $-0.01(0.51)$ & $-0.01(0.50)$ & $0.11(1.35)$ & $0.002(0.19)$ & & & \\
\hline (38) & $-0.09(0.36)$ & $0.19(0.36)$ & $0.11(0.45)$ & $-0.10(1.28)$ & $-0.10(0.54)$ & & & \\
\hline (39) & $0.04(0.35)$ & $-0.02(-0.05)$ & $-0.08(0.21)$ & $0.09(-0.10)$ & $-0.03(0.62)$ & & & \\
\hline (40) & $0.18(0.22)$ & $-0.17(-0.06)$ & $0.08(0.14)$ & & $-0.11(0.69)$ & $0.01(-0.02)$ & & \\
\hline (41) & $-0.08(0.98)$ & $0.13(-0.28)$ & $-0.12(-0.18)$ & $0.10(1.58)$ & $-0.02(0.91)$ & & & \\
\hline (42) & $-0.17(0.07)$ & $0.30(-0.30)$ & $-0.17(0.06)$ & & $0.02(0.59)$ & $0.02(0.59)$ & & \\
\hline (43) & $-0.03(1.72)$ & $0.14(1.07)$ & $-0.16(-0.06)$ & $0.04(0.22)$ & $0.005(0.05)$ & & & \\
\hline (44) & $0.004(1.78)$ & $-0.17(1.04)$ & $0.22(0.01)$ & & $-0.03(0.08)$ & $-0.03(0.08)$ & & \\
\hline
\end{tabular}


(1)
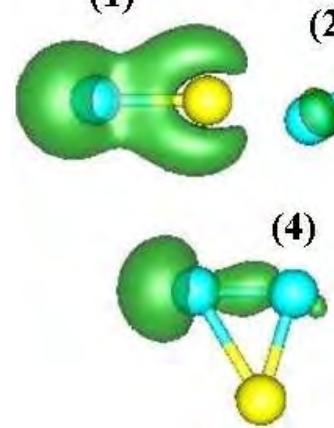

(2)

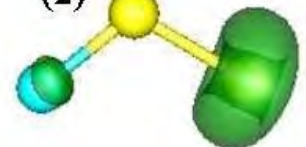

(4)

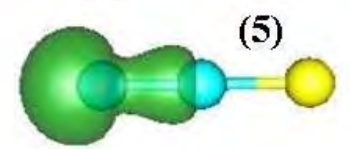

(3)

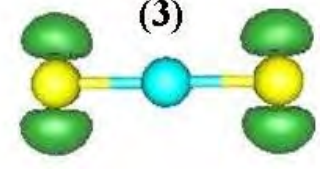

(5)

Figura 6.7. Densidades de espín del dímero y los trímeros. Las superficies se graficaron con un valor de isosuperficie de 0.015 u.a.
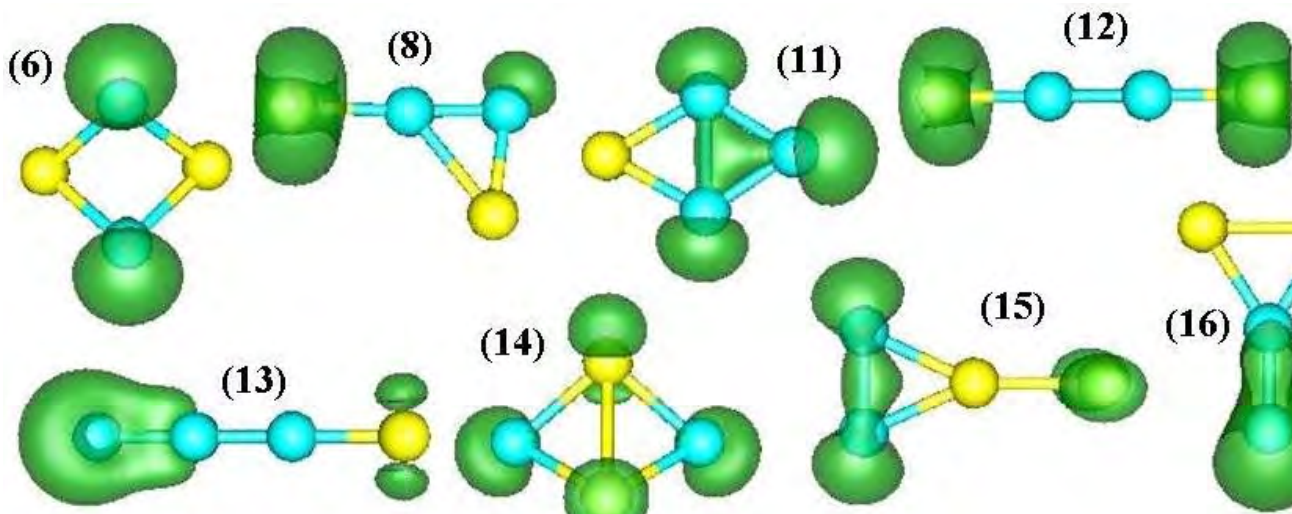

(14)
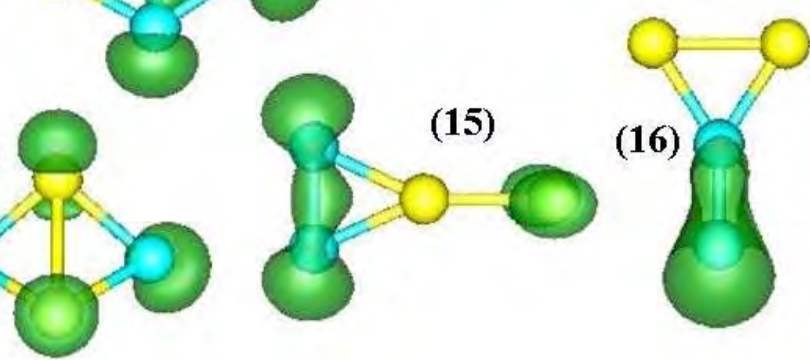

Figura 6.8. Densidades de espín de los tetrámeros de capa abierta. Las superficies se graficaron con un valor de isosuperficie de 0.015 u.a. 


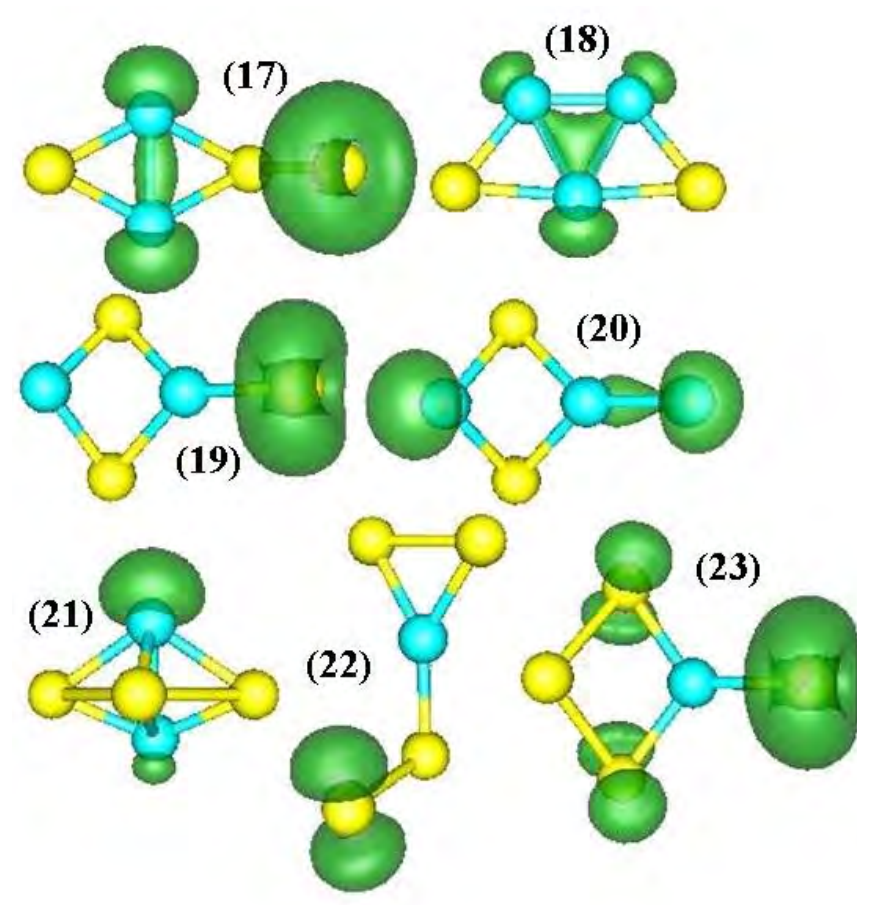

Figura 6.9. Densidades de espín de pentámeros (17) a (23). Las superficies se graficaron con un valor de isosuperficie de 0.015 u.a.

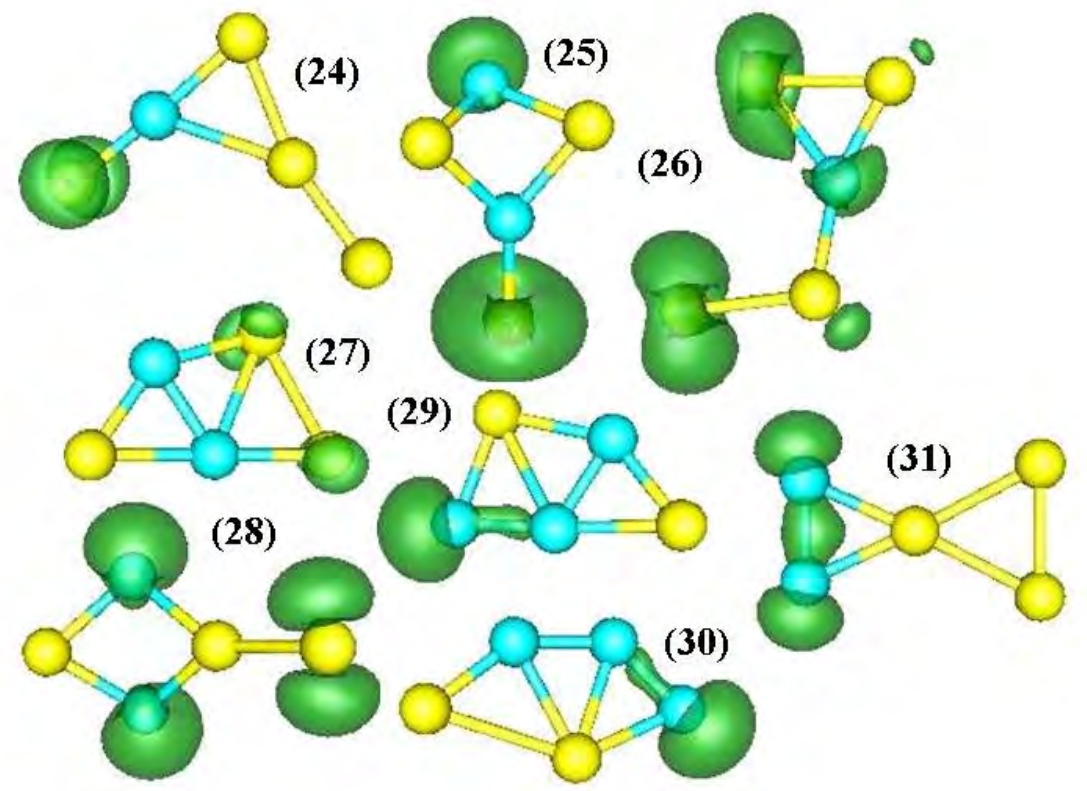

Figura 6.10. Densidades de espín de pentámeros (24) a (31). Las superficies se graficaron con un valor de isosuperficie de 0.015 u.a. 


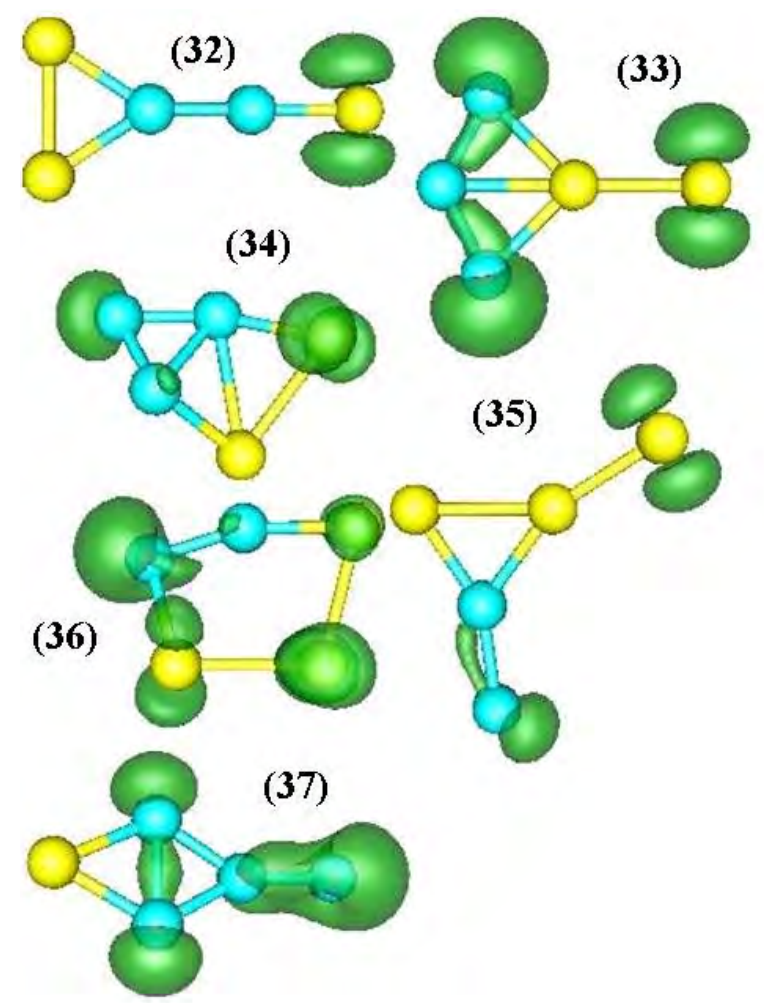

Figura 6.11. Densidades de espín de pentámeros (32) a (37). Las superficies se graficaron con un valor de isosuperficie de 0.015 u.a.

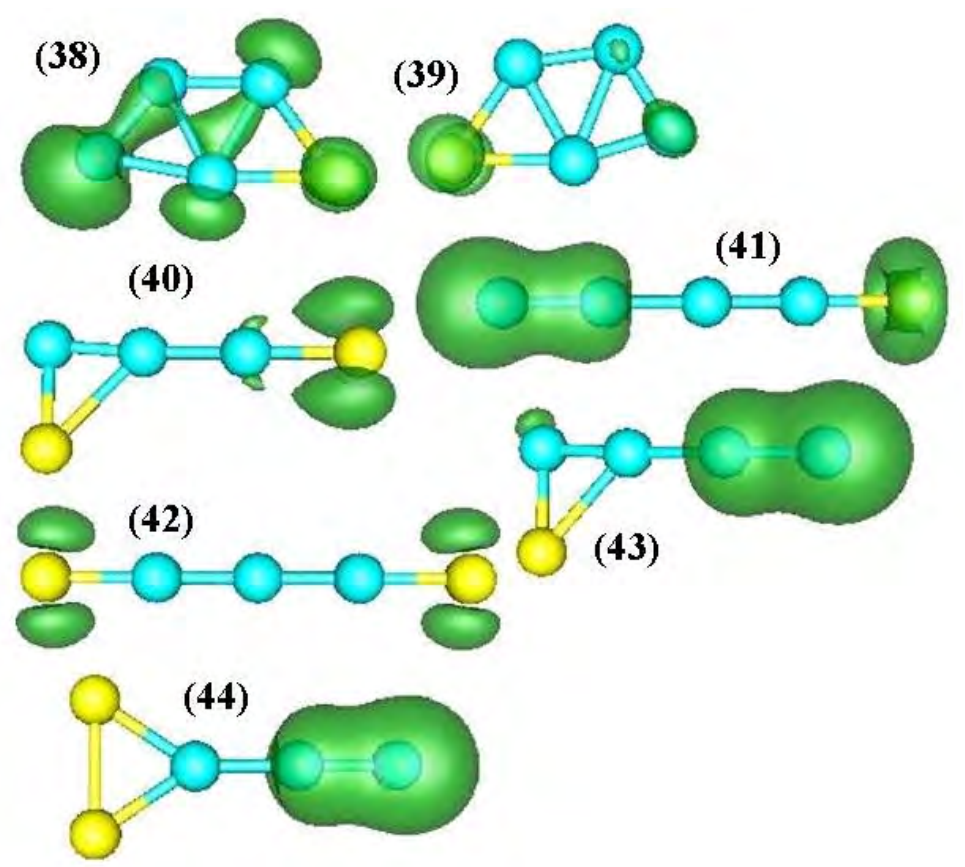

Figura 6.12. Densidades de espín de pentámeros (38) a (44). Las superficies se graficaron con un valor de isosuperficie de 0.015 u.a. 


\subsection{Potenciales electrostáticos moleculares}

En las Figuras 6.13 a 6.1 se muestran los gráficos de potenciales electrostáticos moleculares (MEP) calculados para todos los sistemas. El color azul indica una zona susceptible de ataque electrofílico, mientras que el color rojo muestra una zona susceptible de ataque nucleofílico, los colores verdes y amarillos corresponden a zonas intermedias de valores negativos y positivos muy bajos, respectivamente.

Además, en la Figura 6.13 se muestran los gráficos de MEP correspondientes al dímero y los trímeros junto con los valores de cargas ESP obtenidas para cada átomo, con el objeto de mostrar la buena concordancia entre ellos (Tablas 6.7 a 6.9). Se observa que las cargas ESP son, por sí solas, un buen indicativo de la naturaleza de la zona susceptible de ataque, ya sea nucleofílico o electrofílico.

(1)

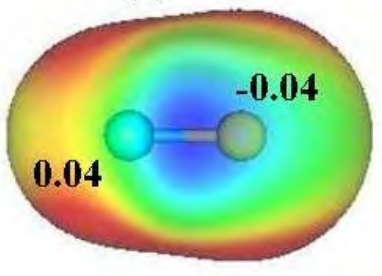

(4)

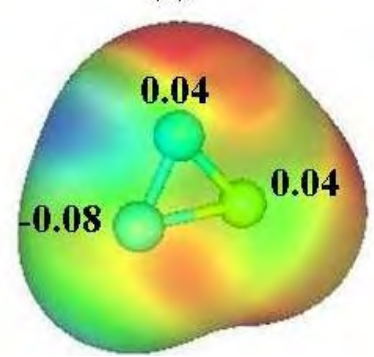

(2)

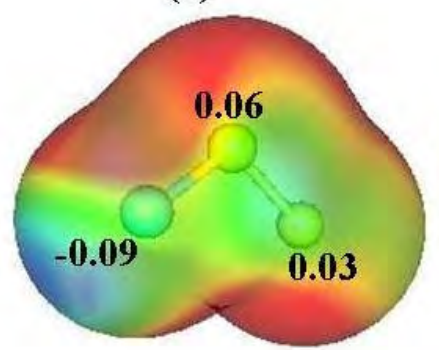

(3)

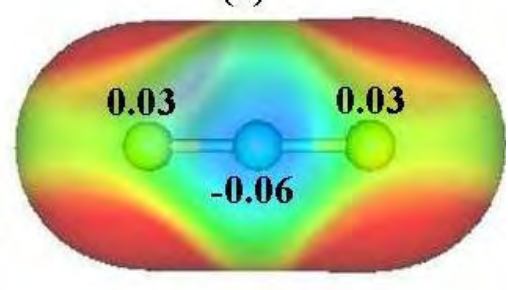

(5)

Figura 6.13. Superficies de potenciales electrostáticos moleculares del dímero y los trímeros. También se muestran las cargas ESP calculadas. Las superficies se graficaron con un valor de isosuperficie de la densidad electrónica de 0.002 u.a.

En el dímero la zona susceptible de ataque electrofílico se sitúa sobre el átomo de fósforo y la zona susceptible de ataque nucleofílico, sobre el átomo de boro. Asimismo, se observa en la Tabla 6.7 y en la Figura 6.7 que el dímero presenta una mayor densidad de espín en el átomo de boro. En los trímeros los átomos de fósforo presentan cargas ESP de carácter positivo, mostrando zonas susceptibles de ataque nucleofílico, con excepción del P1 en el trímero (5), que presenta una carga ESP negativa muy pequeña. Por otro lado, los átomos de boro de los trímeros muestran un carácter susceptible de ataque electrofílico, con excepción de B2 en (4) y B1 en (5). Con respecto a la densidad de espín en los trímeros, los mayores valores se presentan en átomos de boro con cargas ESP positivas, con excepción del trímero (4) (Tabla 6.7 , Figuras 6.7 y 6.13$)$. 

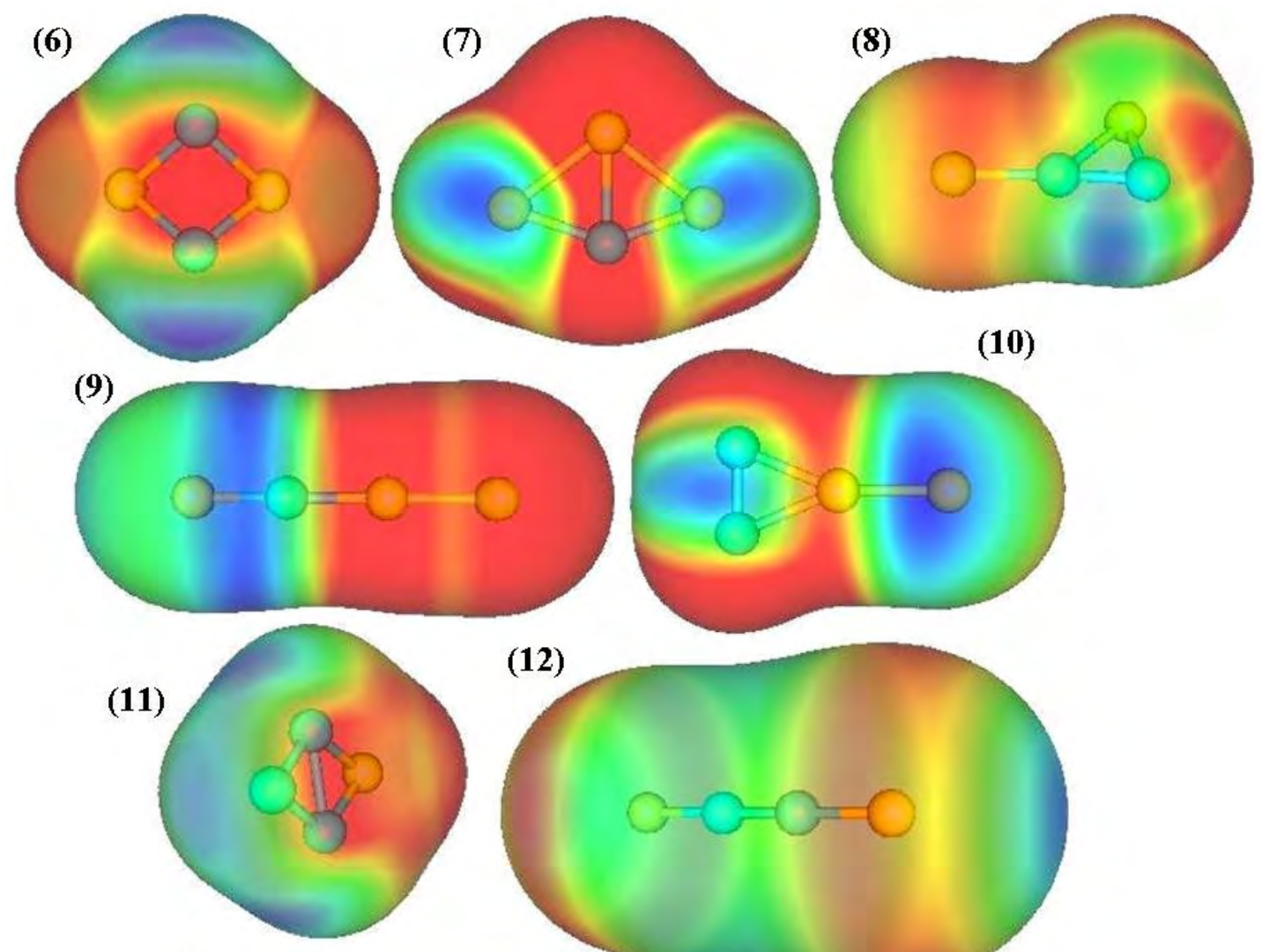

(13)

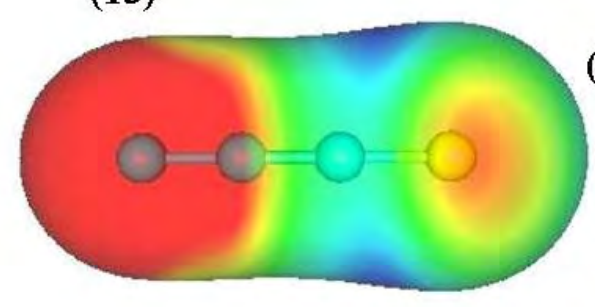

(12)

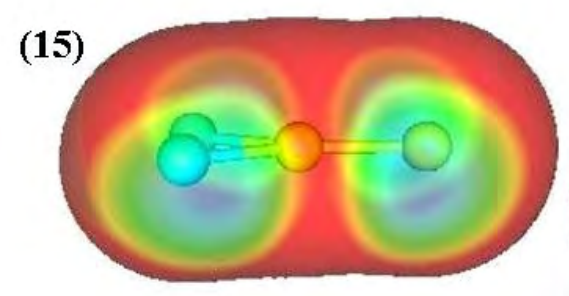

(14)

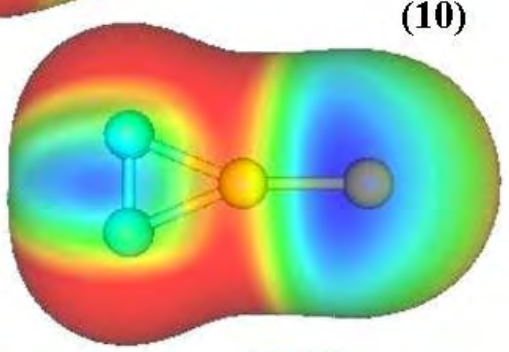

(10)
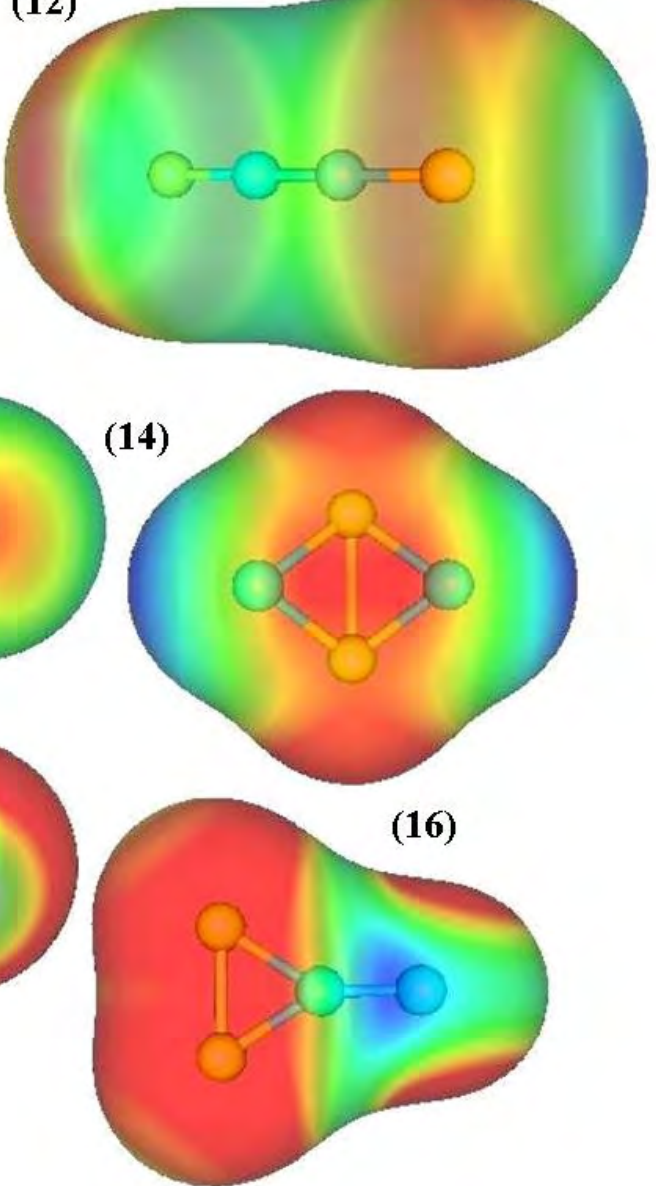

Figura 6.14. Superficies de potenciales electrostáticos moleculares para los tetrámeros. Las superficies se graficaron con un valor de isosuperficie de la densidad electrónica de 0.002 u.a., con excepción de (12), que se graficó con un valor de de 0.0002 u.a.

En los tetrámeros no se observa una tendencia a presentar cargas ESP negativas o positivas en algún tipo de átomo. Sin embargo, se evidencia una tendencia a presentar una alta densidad de espín en aquellos átomos que poseen cargas ESP negativas, por lo que las zonas de ataque 
electrofílico podrían deberse a electrones desapareados (Tabla 6.8, Figuras 6.8 y 6.14). Por otro lado, el tetrámero más estable, (11), presenta una carga ESP negativa en un átomo de boro (B1), una alta densidad de espín en ese mismo átomo y cargas ESP positivas bajas en demás átomos de boro.
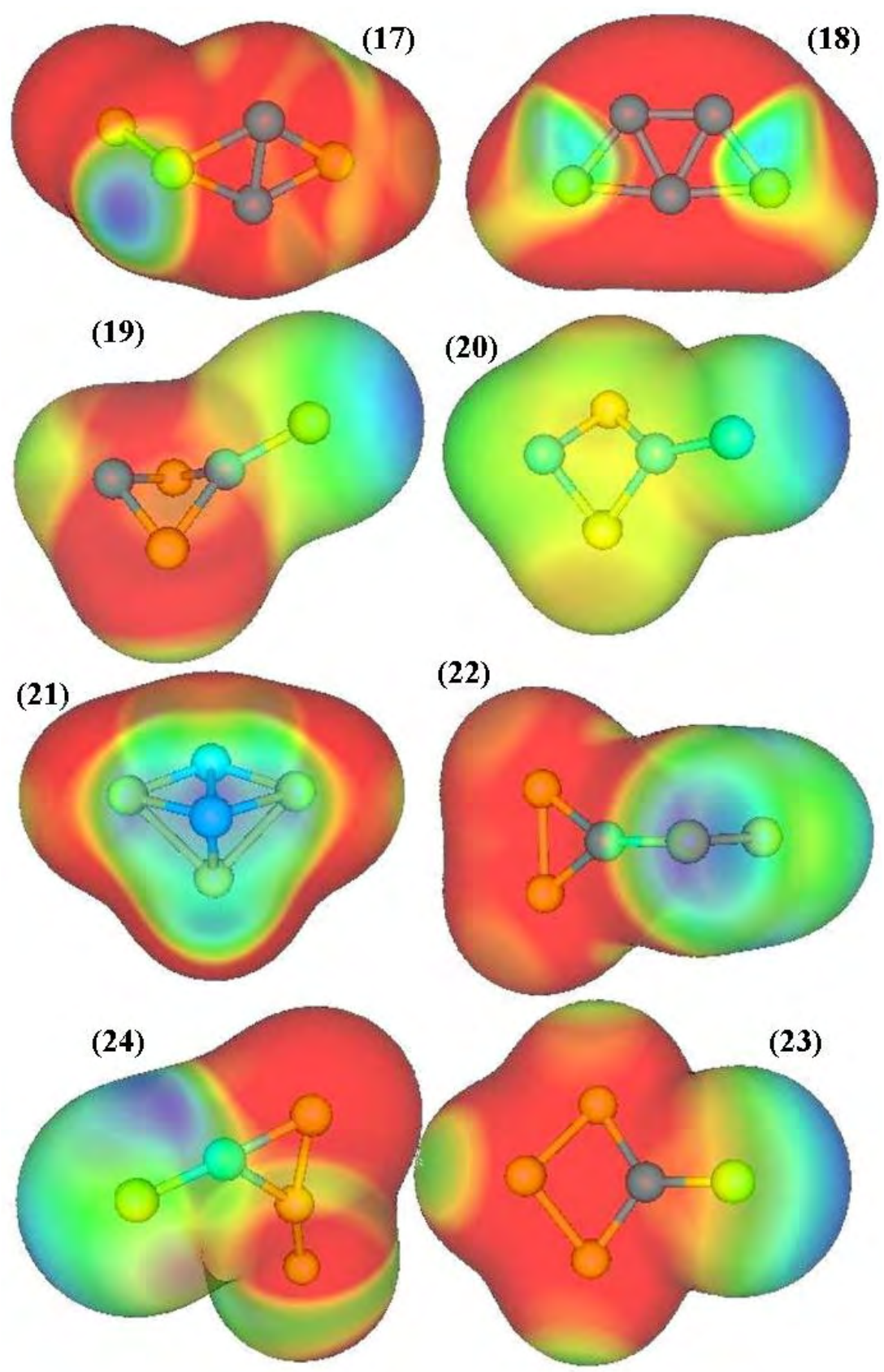

Figura 6.15. Superficies de potenciales electrostáticos moleculares para los pentámeros (17) a (23). Las superficies se graficaron con un valor de isosuperficie de la densidad electrónica de 0.002 u.a. 


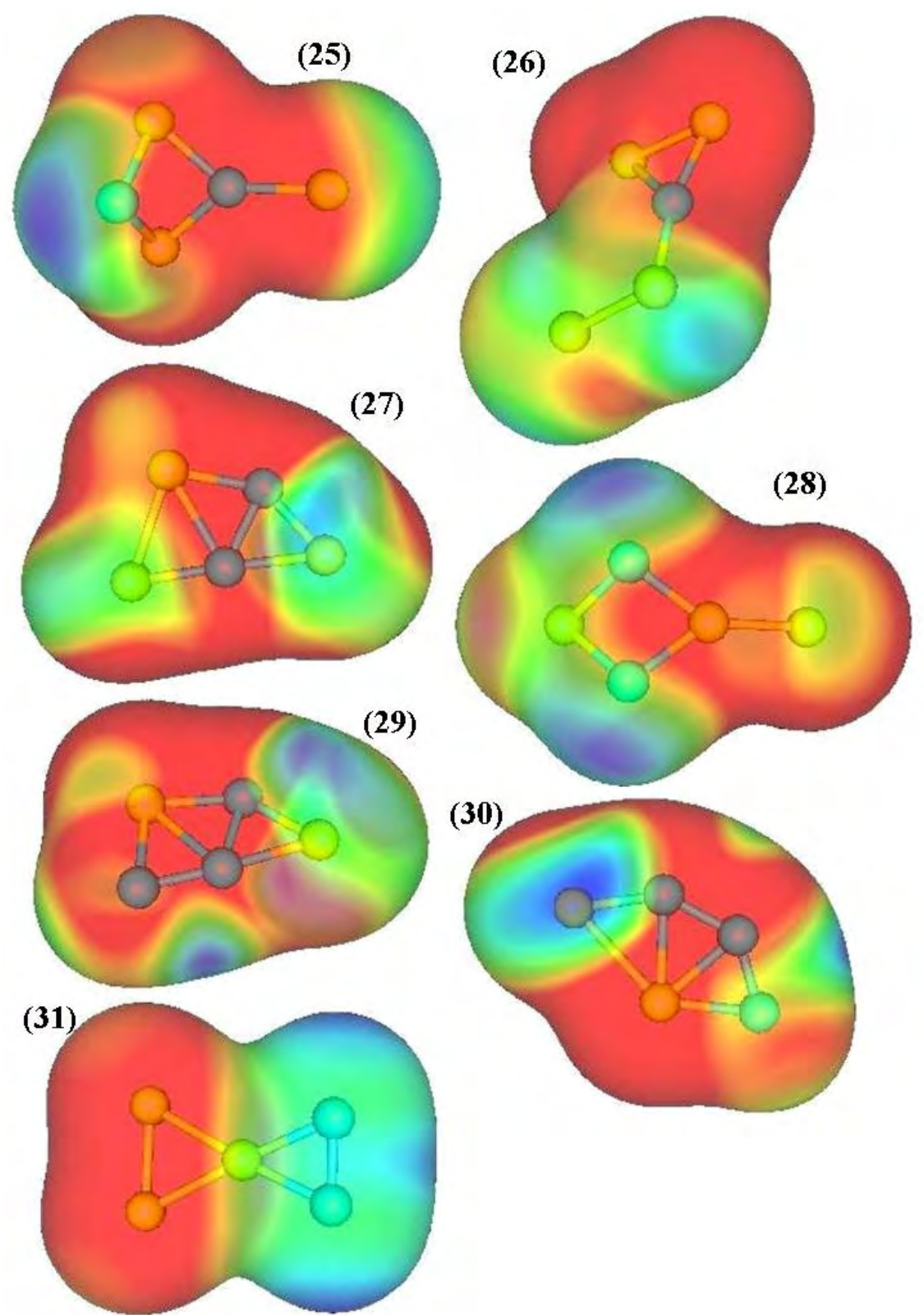

Figura 6.16. Superficies de potenciales electrostáticos moleculares para los pentámeros (25) a (31). Las superficies se graficaron con un valor de isosuperficie de la densidad electrónica de 0.002 u.a. 


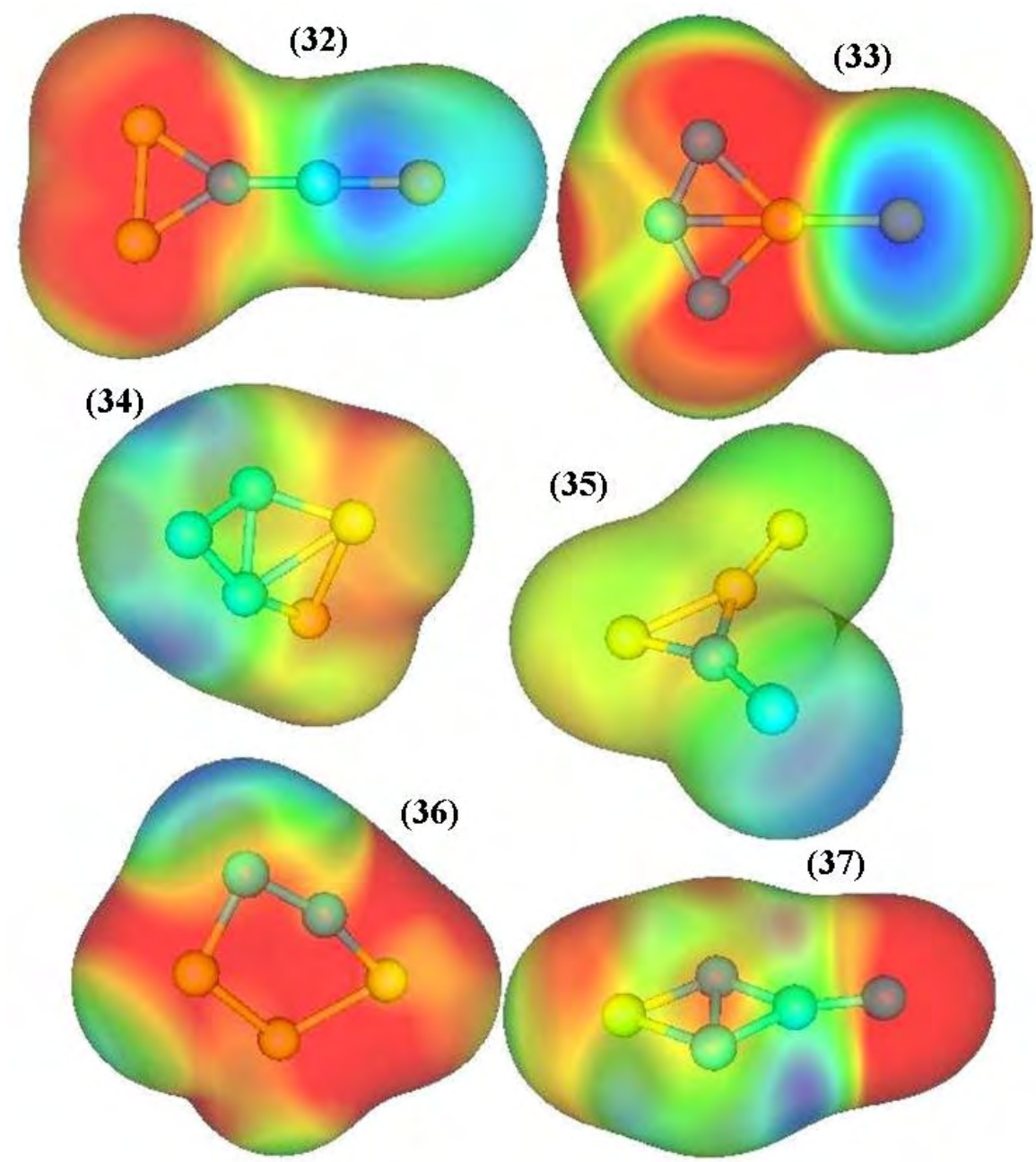

Figura 6.17. Superficies de potenciales electrostáticos moleculares para los pentámeros (37) a (44). Las superficies se graficaron con un valor de isosuperficie de la densidad electrónica de 0.002 u.a. 


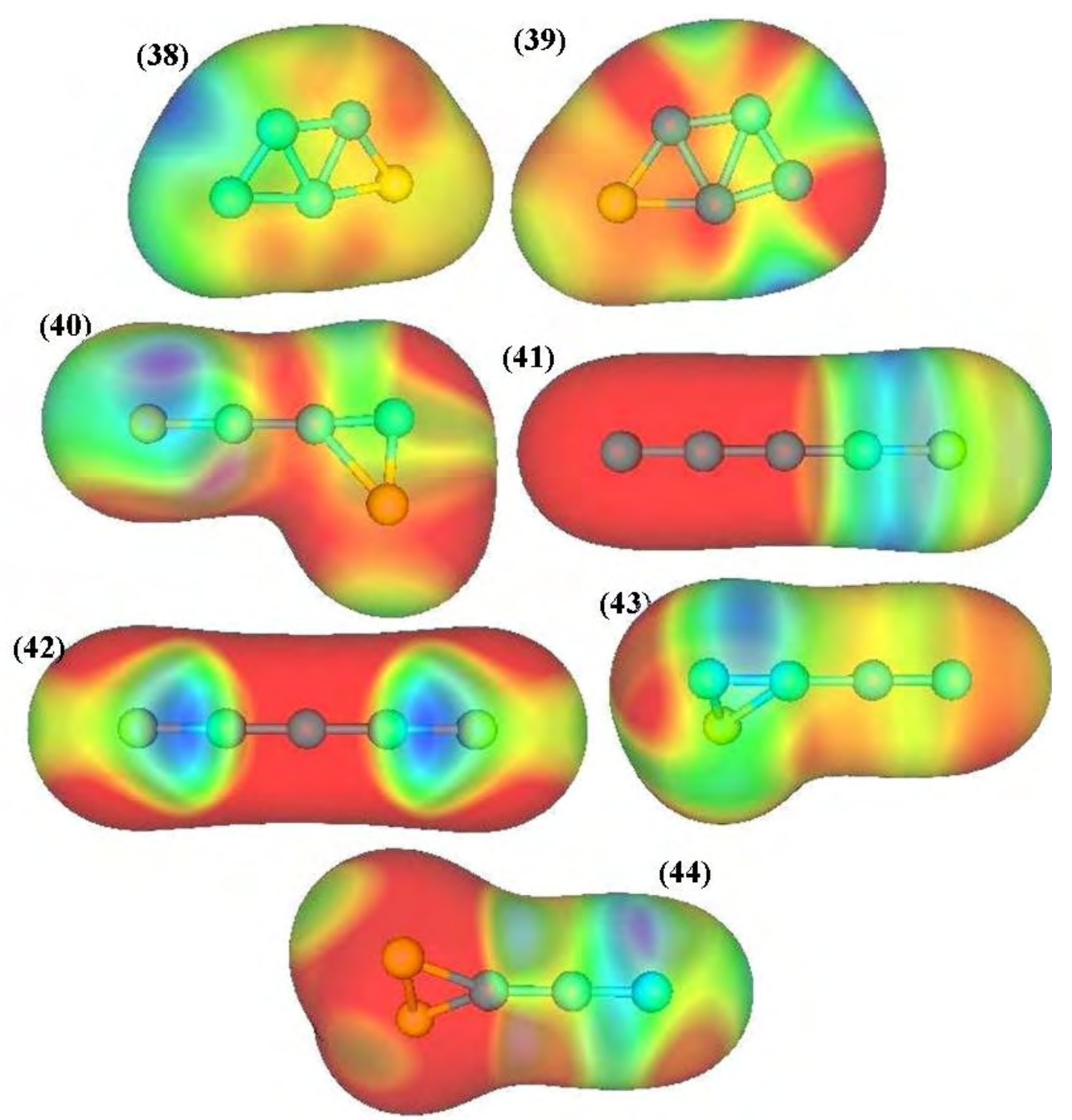

Figura 6.18. Superficies de potenciales electrostáticos moleculares para los pentámeros (38) a (44). Las superficies se graficaron con un valor de isosuperficie de la densidad electrónica de 0.002 u.a.

No se evidencia una tendencia de los pentámeros de poseer zonas susceptibles de ataque electrofílico en átomos de boro o de fósforo. Se observa, sin embargo, que en la mayoría de los casos en que un átomo posee densidad de espín alta (mayor a 0.40 u.a.), también posee carga ESP negativa, siendo susceptible de ataque electrofílico y esa susceptibilidad se debe a electrones desapareados (Tabla 6.9, Figuras 6.9 a 6.12 y 6.15 y 6.18).

\subsection{Patrones de crecimiento}

A continuación se muestran los patrones de crecimiento obtenidos. Estos patrones se construyen a partir del dímero (1) agregando átomos de $\mathrm{P}$ y de $\mathrm{B}$, siguiendo el camino de aumento de la energía de atomización, como se expresó con anterioridad. Se plantea el patrón de crecimiento más probable para agregados binarios constituidos por $\mathrm{B}$ y $\mathrm{P}$.

Los patrones de crecimiento que involucran al agregado (2) se muestran en las Figuras 6.19.a a 6.19.e y 6.21 . 


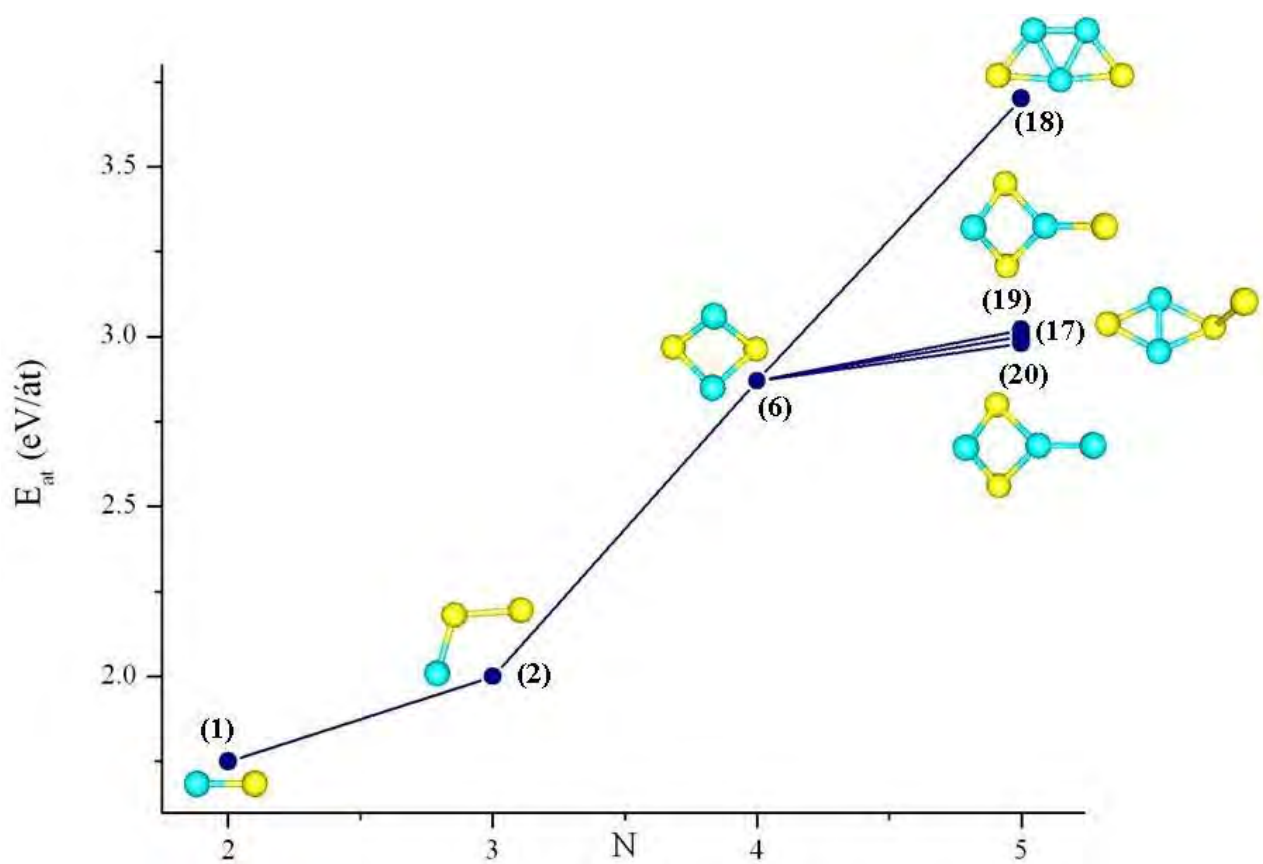

Figura 6.19.a Patrón de crecimiento del trímero (2) y el tetrámero (6).

En la Figura 19.a se presenta el patrón de crecimiento obtenido para el trímero (2) y el tetrámero (6). Se observa que para obtener (2) a partir de (1), un átomo de fósforo se une a otro átomo de fósforo (P1), por medio de un ataque electrofílico a una zona con electrones desapareados (Tabla 6.7, Figuras 6.7 y 6.13). Se continúa el crecimiento y se obtiene el agregado romboidal alternado, (6), al unir un átomo de boro al enlace P1-P2 en (2), una zona susceptible de ataque nucleofílico con electrones desapareados (Tabla 6.8, Figuras 6.7 y 6.13). La formación del pentámero (18) se ve favorecida, debido a su alta energía de atomización, con respecto a los demás pentámeros involucrados en el crecimiento. Este pentámero se forma mediante el ingreso de un átomo de boro a un enlace B-P, obteniéndose un pentámero altamente estable, con mayoría de átomos cargados negativamente y electrones desapareados sobre todos los átomos (Tabla 6.9 y Figuras 6.9 y 6.15). Por otro lado, la formación de (19) se realiza mediante la unión de un átomo de fósforo realizando un ataque electrofílico a un átomo de boro de (6). Asimismo, (17) se obtiene por la unión de un átomo de fósforo a una zona susceptible de ataque nucleofilico. Las energías de atomización de (17) y (19) son muy similares (Tabla 6.5), por lo que se postula que su formación es igualmente probable. Finalmente, (20) se forma cuando un átomo de boro ataca de manera electrofílica en un átomo de boro de (6). 


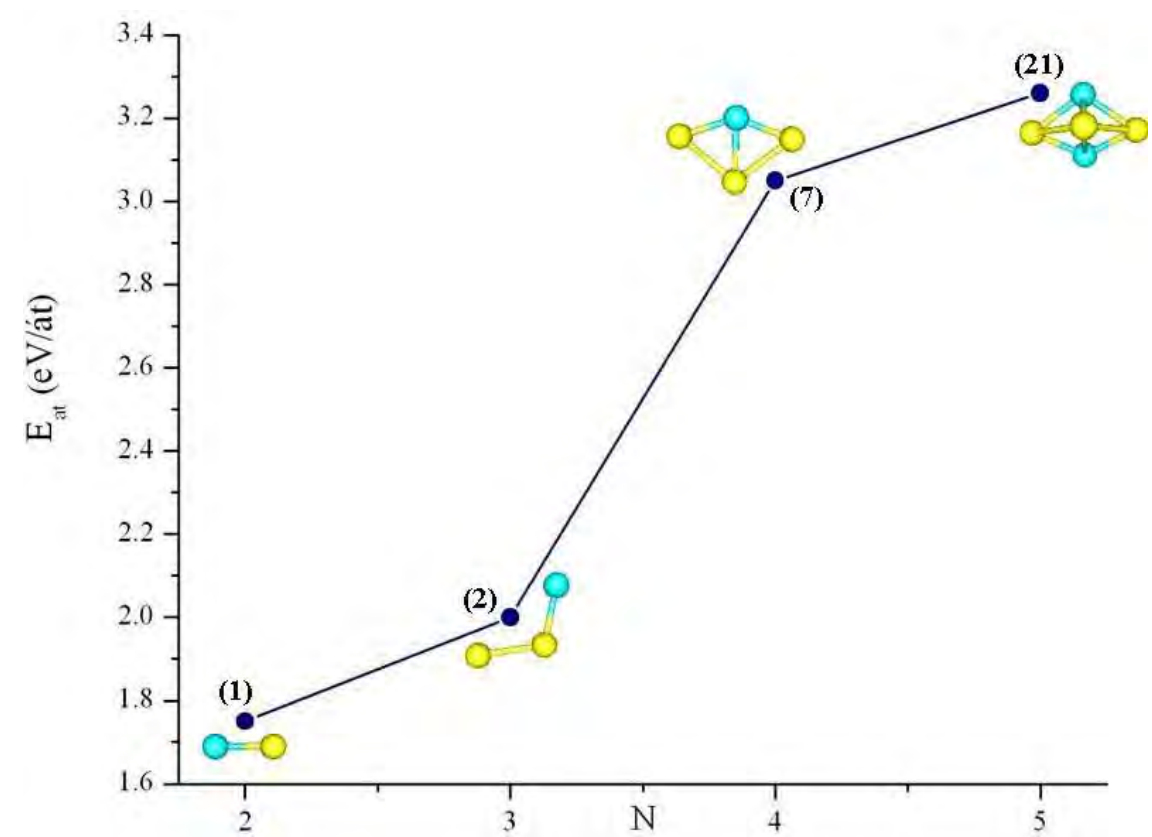

Figura 6.19.b Patrón de crecimiento del trímero (2) y el tetrámero (7).

En la Figura 6.19.b se muestra el camino de crecimiento del trímero (2) y el tetrámero (7). La formación del trímero se describió con anterioridad (Figura 6.19.a). La obtención de (7) se realiza mediante la unión de un átomo de fósforo a una zona susceptible de ataque electrofílico, B1 de (2) (Figuras 6.1, 6.7 y 6.13, Tabla 6.7). El pentámero (21) se obtiene mediante un ataque nucleofílico de un átomo de boro a P2 de (7) (Tabla 6.8, Figuras 6.2, 6.8 y 6.14 ), produciendo un agregado de cinco átomos con una $\mathrm{E}_{\mathrm{at}}$ considerablemente alta (Tabla 6.5), donde las zonas susceptibles de ataque electrofílico y los electrones desapareados se sitúan en átomos de boro (Tabla 6.9, Figuras 6.9 y 6.15).

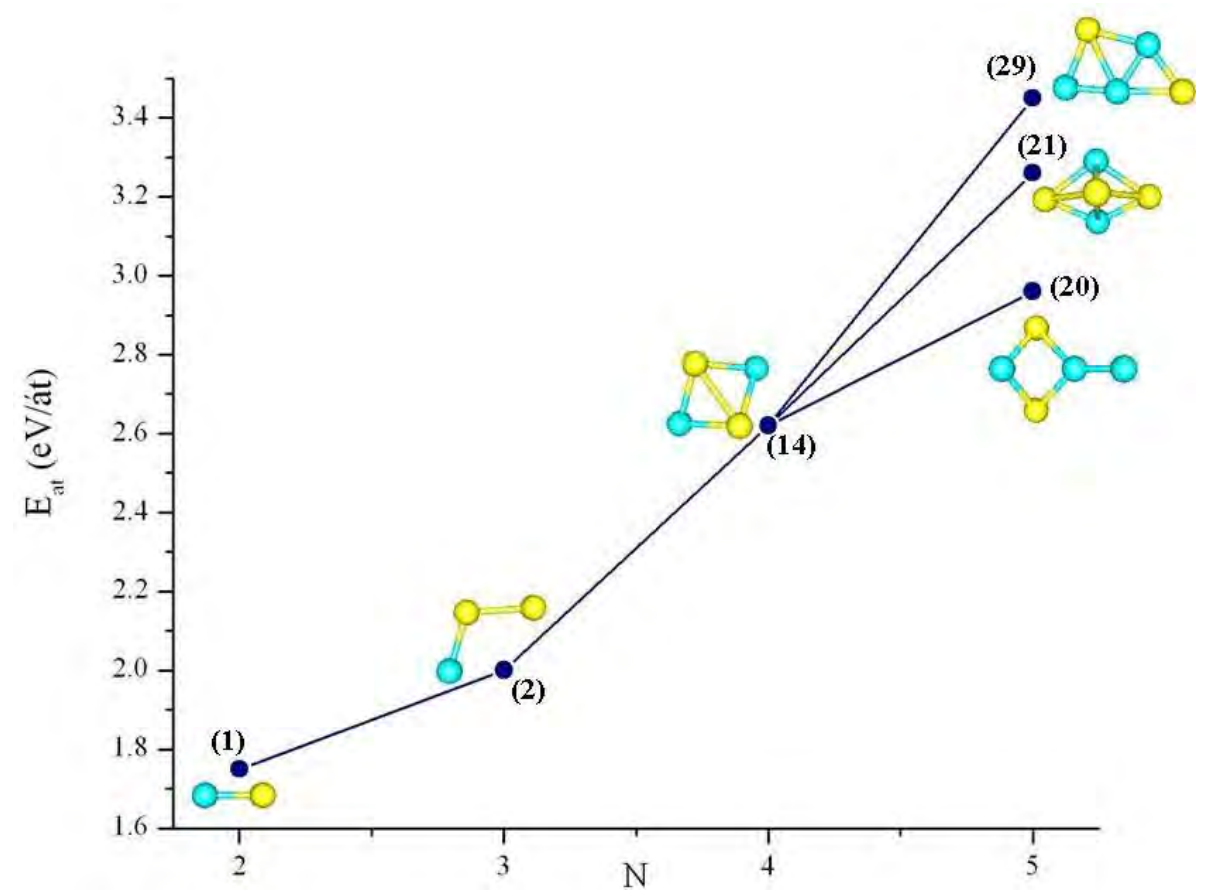

Figura 6.19.c. Patrón de crecimiento del trímero (2) y el tetrámero (14). 
En la Figura 6.19.c se presenta el patrón de crecimiento obtenido para el trímero (2) y el tetrámero (14). La formación de (2) a partir del dímero BP se describe en la Figura 6.19.a. La obtención del tetrámero (14) a partir de (2), se realiza cuando un átomo de boro se une a una zona susceptible de ataque nucleofílico, como son los átomos de fósforo en (2) (Tabla 6.7, Figura 6.13). Igualmente, el ataque nucleofílico de un átomo de boro a un fósforo de (14), para formar (29), es más probable que la unión de un átomo de fósforo en la misma zona, para formar (21); y que el ataque de un átomo de boro de manera electrofílica para formar (20) (Tabla 6.8, Figura 6.15 y 6.16).

El pentámero más estable en el patrón de crecimiento mostrado en la Figura 6.19.c, (29), presenta alta densidad de espín en un átomo de boro susceptible de ataque electrofílico $\mathrm{y}$, de igual manera, los átomos de fósforo presentan cargas ESP negativas (Tabla 6.9, Figuras 6.10 y 6.16).

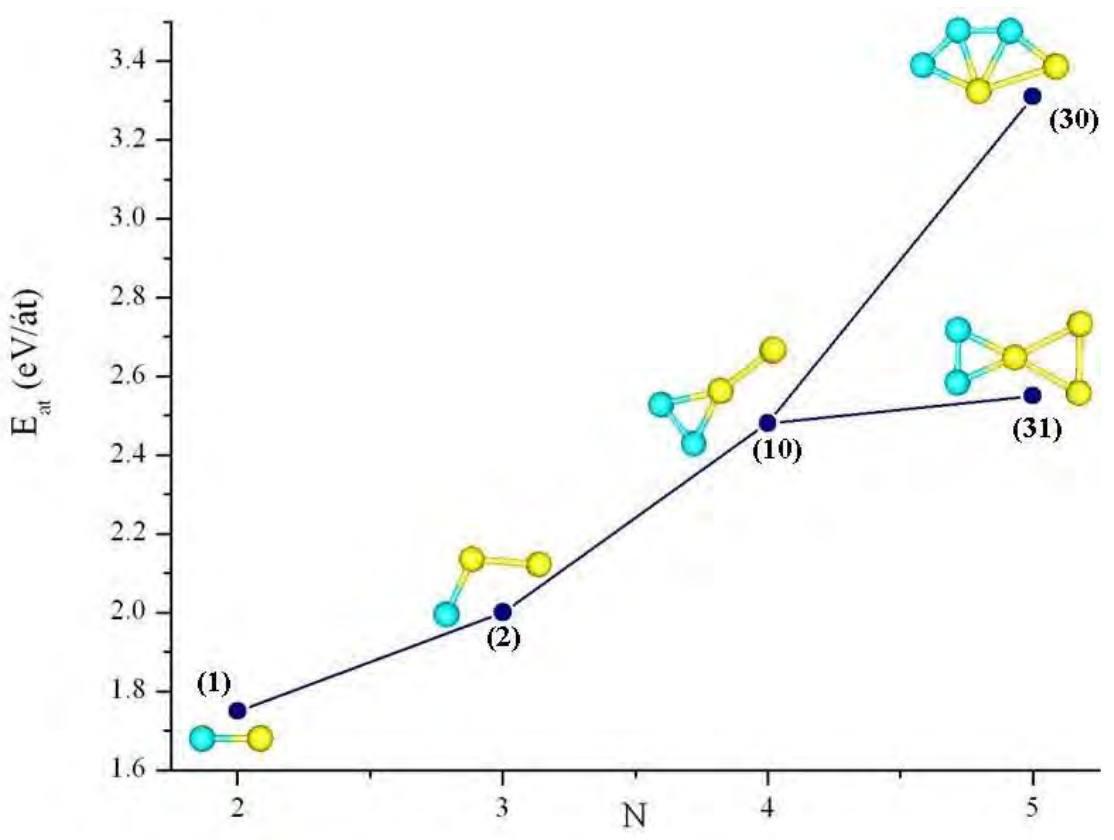

Figura 6.19.d Patrón de crecimiento del trímero (2) y el tetrámero (10).

En la Figura 6.19.d se presenta el camino de crecimiento del tetrámero (10), el cual proviene del trímero (2). La obtención del trímero (2) se presentó con anterioridad en la Figura 6.19.a. Para la obtención de (10) a partir del trímero, se produce un ataque nucleofílico por parte de un átomo de boro (Tabla 6.7, Figura 6.13). Por otro lado, la formación del pentámero (30) resulta más probable, debido a que éste posee mayor $\mathrm{E}_{\mathrm{at}}$. Este pentámero se obtiene mediante la unión de un átomo de boro a P1 de (10) en un ataque nucleofílico (Tabla 6.8, Figura 6.14). La obtención de (31), por otro lado, se realiza mediante el ataque nuclefílico de un átomo de fósforo a la misma zona, resultando en una energía de atomización más baja.

Asimismo, el pentámero más estable, (30), presenta sólo dos átomos de boro y uno de fósforo con cargas ESP negativas (Tabla 6.8, Figura 6.16). 


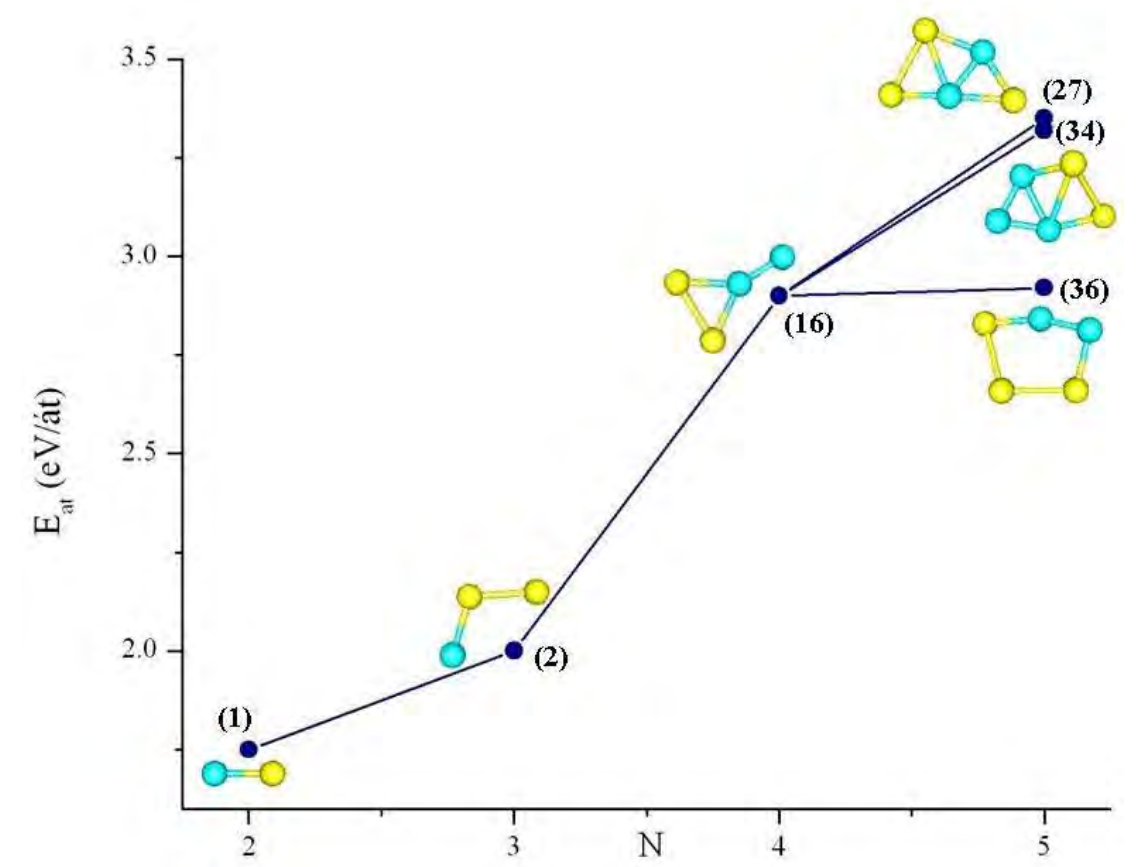

Figura 6.19.e Patrón de crecimiento del trímero (2) y el tetrámero (16).

En la Figura 6.19.e se presenta el camino de crecimiento obtenido para el tetrámero (16) a partir del trímero (2), cuya obtención desde (1) se explica en el texto que acompaña a la Figura 6.19.a. La obtención de (16) desde el trímero, se produce por el ataque nucleofílico de un átomo de boro a otro, B1 (Tabla 6.7, Figura 6.13). Continuando el crecimiento para formar los pentámeros, resulta ser (27) el de mayor $\mathrm{E}_{\mathrm{at}}$, que se constituye por la unión de un átomo de fósforo a B2 de (16), en un ataque electrofílico, de mayor probabilidad que el ataque electrofílico por parte de un átomo de B para obtener (34) (Tabla 6.8, Figura 6.14). Asimismo, la formación de (36) a partir del tetrámero y mediante un ataque nucleofílico de un fósforo dirigido a los átomos P1 y P2 (Tabla 6.8, Figura 6.14), resulta en un pentámero de mucha menor $\mathrm{E}_{\text {at }}$ que (27) (Tabla 6.5).

En las Figuras 6.20.a, 6.20.b y 6.23 se exponen los patrones de crecimiento que involucran al trímero (5). 


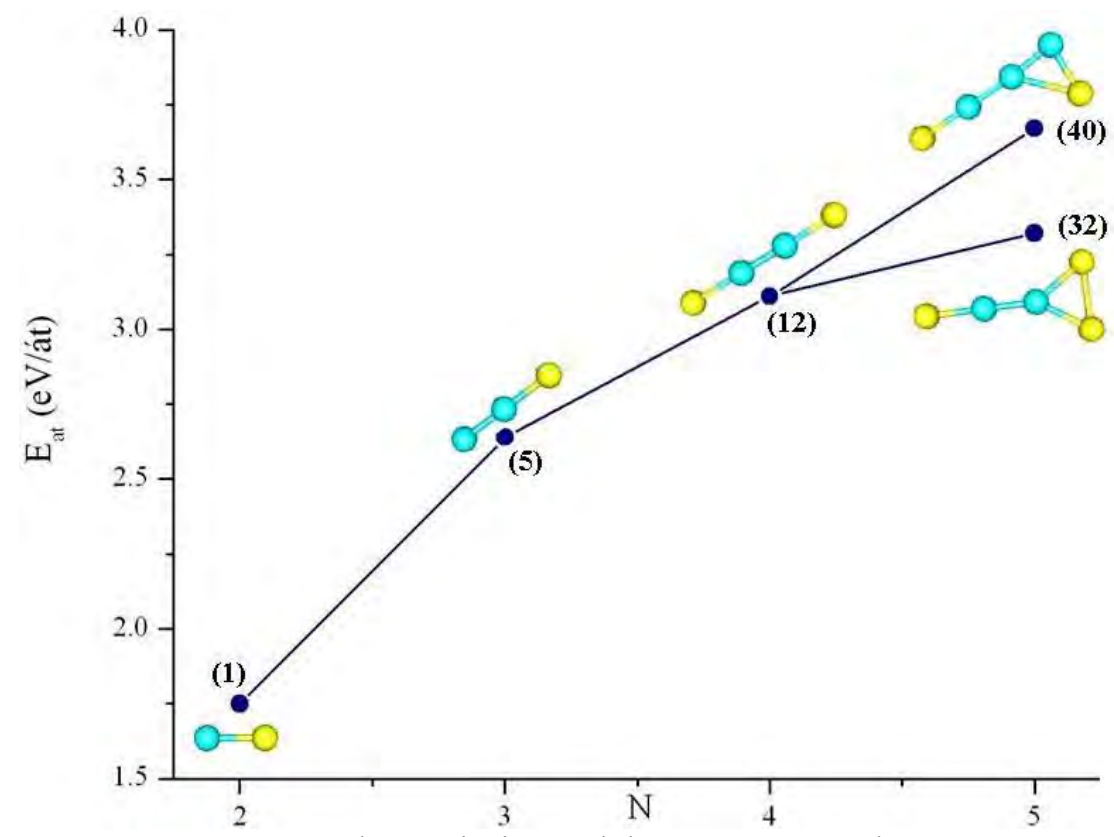

Figura 6.20.a Patrón de crecimiento del trímero (5) y el tetrámero (12).

En la Figura 6.20.a se muestra el patrón de crecimiento del tetrámero (12) a partir del trímero (5). La formación de (5) se produce cuando un átomo de boro se une por medio de un ataque nucleofílico a B1 del dímero, formando un trímero lineal (Tabla 6.7, Figuras 6.1 y 6.13). Asimismo, el tetrámero se obtiene por medio de un ataque nucleofílico de un átomo de fósforo a B1 de (5) (Tabla 6.7, Figura 6.13). Luego, la obtención de los pentámeros se ve favorecida hacia un agregado donde un átomo de boro ataca sobre una zona susceptible de ataque nucleofílico, (40), más que la unión de un átomo de fósforo a la misma zona (32) (Tabla 6.8, Figura 6.14).

El pentámero más estable, (40), presenta sólo dos átomos con cargas ESP negativas (Tabla 6.9, Figura 6.18), B2 y P1; y una densidad de espín alta localizada en P1. 


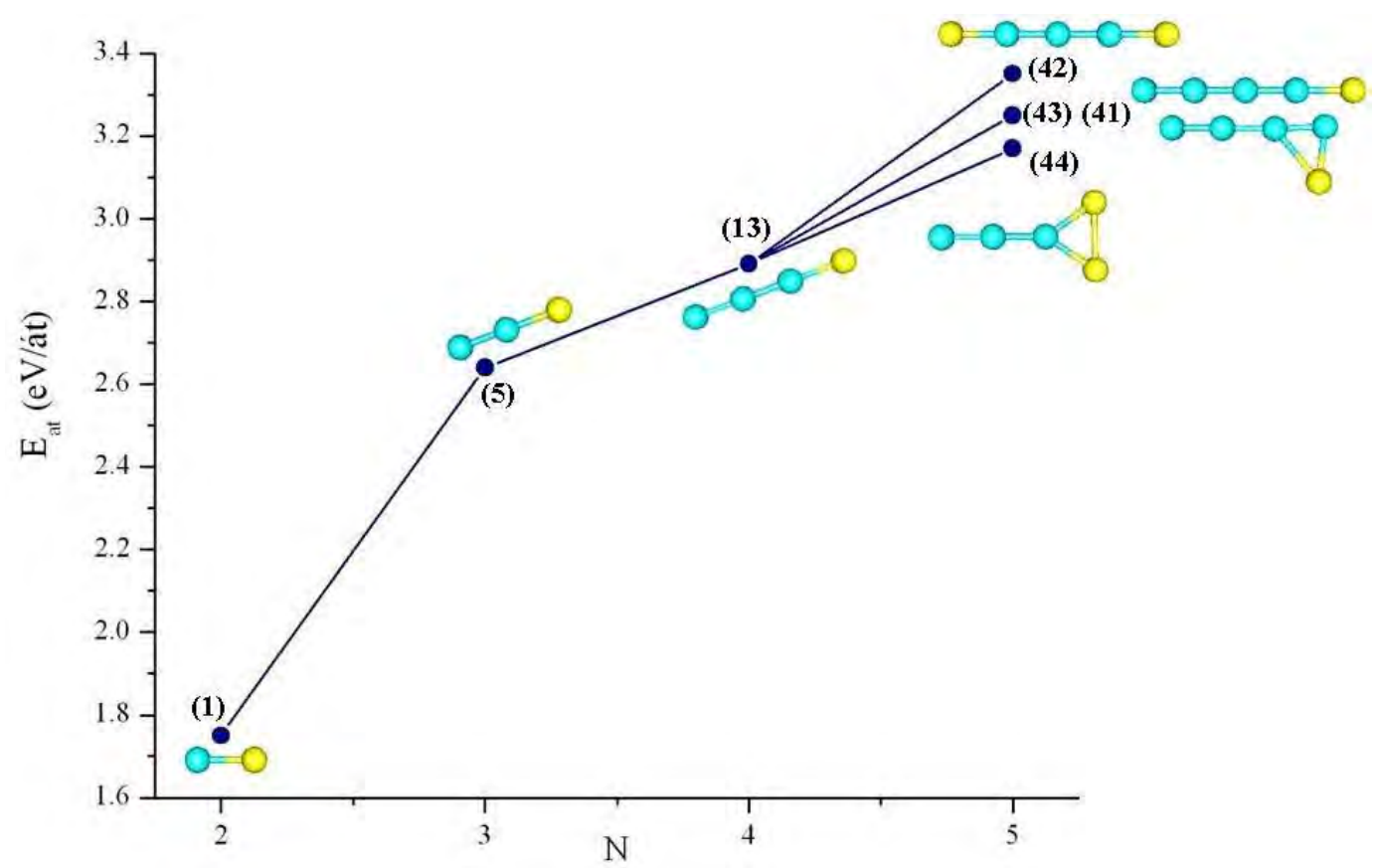

Figura 6.20.b Patrón de crecimiento del trímero (5) y el tetrámero (13).

En la Figura 6.20.b se expone el camino de crecimiento del tetrámero (13) a partir del trímero (5). La obtención de (5) se describe en el texto que acompaña a la Figura 6.20.a. El camino de crecimiento continúa hacia (13) por medio de un ataque nucleofílico de un átomo de boro hacia otro átomo de boro, B1 en (5) (Tabla 6.7, Figura 6.13). El pentámero más estable es (42), donde un átomo de fósforo actúa como electrófilo y se une a un átomo de boro de (13) (Tabla 6.8, Figura 6.14). Para la formación de (41) y (43), un átomo de boro actúa como electrófilo y se forman dos agregados de energía muy similar, aunque estructura diferente (Figura 6.6, Tabla 6.5). El agregado de menor energía de atomización es (44), donde un átomo de fósforo se une actuando como electrófilo.

Teniendo en cuanta lo presentado en la Tabla 6.9 y las Figuras 6.18 y 6.12, se evidencia que el agregado (42) posee cargas ESP negativas sobre los átomos de boro y densidad de espín altas situadas en átomos de fósforo. 


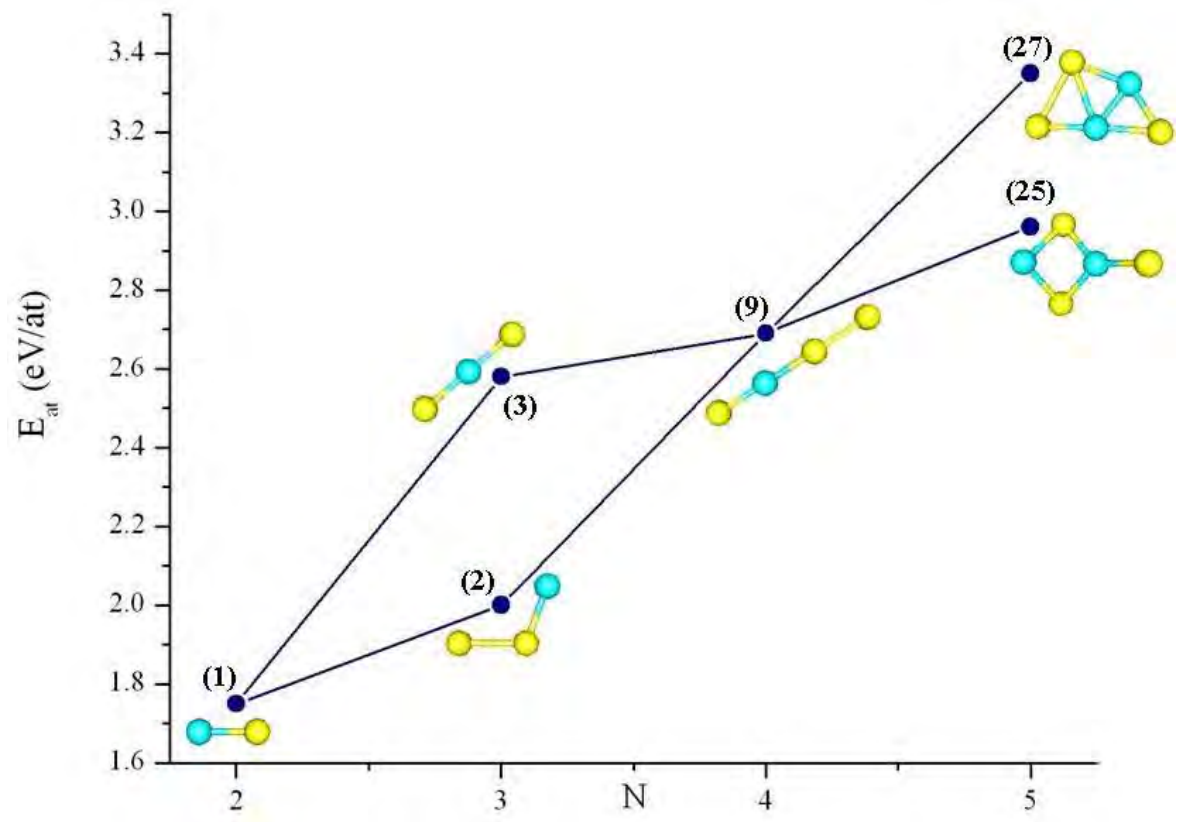

Figura 6.21. Patrón de crecimiento de los trímeros (2) y (3) y el tetrámero (9).

En la Figura 6.21 se muestra el camino de crecimiento obtenido para el tetrámero (9), cuya formación puede presentarse tanto a partir del trímero (2), como de (3). La obtención de (2) se describe en la Figura 6.19.a. Para la formación del agregado (3), un átomo de fósforo se une a una zona susceptible de ataque nucleofílico (Tabla 6.7, Figura 6.13), formando un agregado de mayor $\mathrm{E}_{\text {at }}$ que (2) y, por consecuencia, más estable y de mayor probabilidad de formación. Asimismo, para formar el tetrámero (9) a partir de (3), un átomo de fósforo se une a otro átomo de fósforo por medio de un ataque nucleofílico a una zona de electrones desapareados (Tabla 6.7, Figuras 6.7 y 6.13). Por el contrario, para obtener (9) a partir de (2), un átomo de fósforo ataca una zona susceptible de ataque nucleofílico (B1 en (2)).

Con respecto a la formación de los pentámeros, la unión de un átomo de boro a una zona susceptible de ataque electrofílico (B1 en (9)), es más probable que la unión de un átomo de B a un átomo de fósforo para formar (25), en un ataque electrofílico, P3 en (9) (Tabla 6.8, Figura 6.14). Teniendo en cuenta lo informado en la Tabla 6.9 y la Figura 6.16, es más probable formar un pentámero cuyos átomos de boro sean zonas susceptibles de ataque nucleofílico, como en (27), que un pentámero que posea sólo un átomo de boro con carga ESP negativa, como es el caso de (25). 


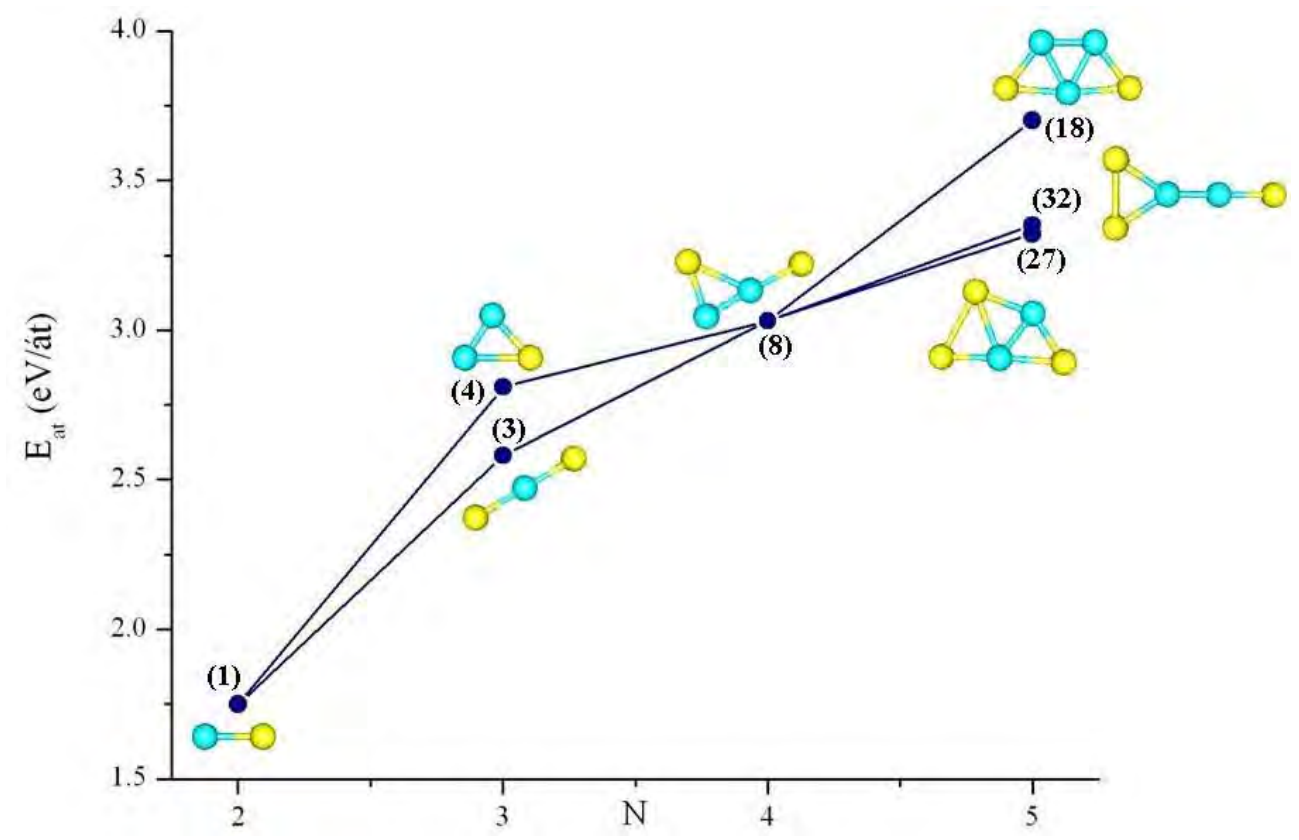

Figura 6.22. Patrón de crecimiento de los trímeros (3) y (4) con el tetrámero (8).

La Figura 6.22 muestra el camino de crecimiento de los agregados (3) y (4) con el tetrámero (8). La obtención de (3) a partir de (1) ya se describió con anterioridad (Figura 6.21). El crecimiento de (1) hacia (4), de mayor energía de atomización, resulta de la unión de un átomo de boro en un ataque nucleofílico en (1), Tabla 6.7 y Figura 6.13. Asimismo, el crecimiento hacia (8) se continua por el enlace de un átomos de fósforo a una zona susceptible de ataque electrofílico (B1 en (4)). Para la formación de los pentámeros, resulta más probable la unión de un átomo de boro a otro boro (B1 de (8)), susceptible de ataque electrofílico, que la unión de un fósforo a otro átomo de boro con carga ESP negativa muy pequeña (B2 de (8)) o que la unión de un átomo de fósforo a B1 de (8) (Tabla 6.8 y Figura 6.14). El resultado es un pentámero, (18), con los átomos de fósforo con electrones desapareados y cargas ESP negativas y, también, dos de los tres átomos de boro susceptibles de ataque electrofílico (Tabla 6.9, Figuras 6.9 y 6.15). 


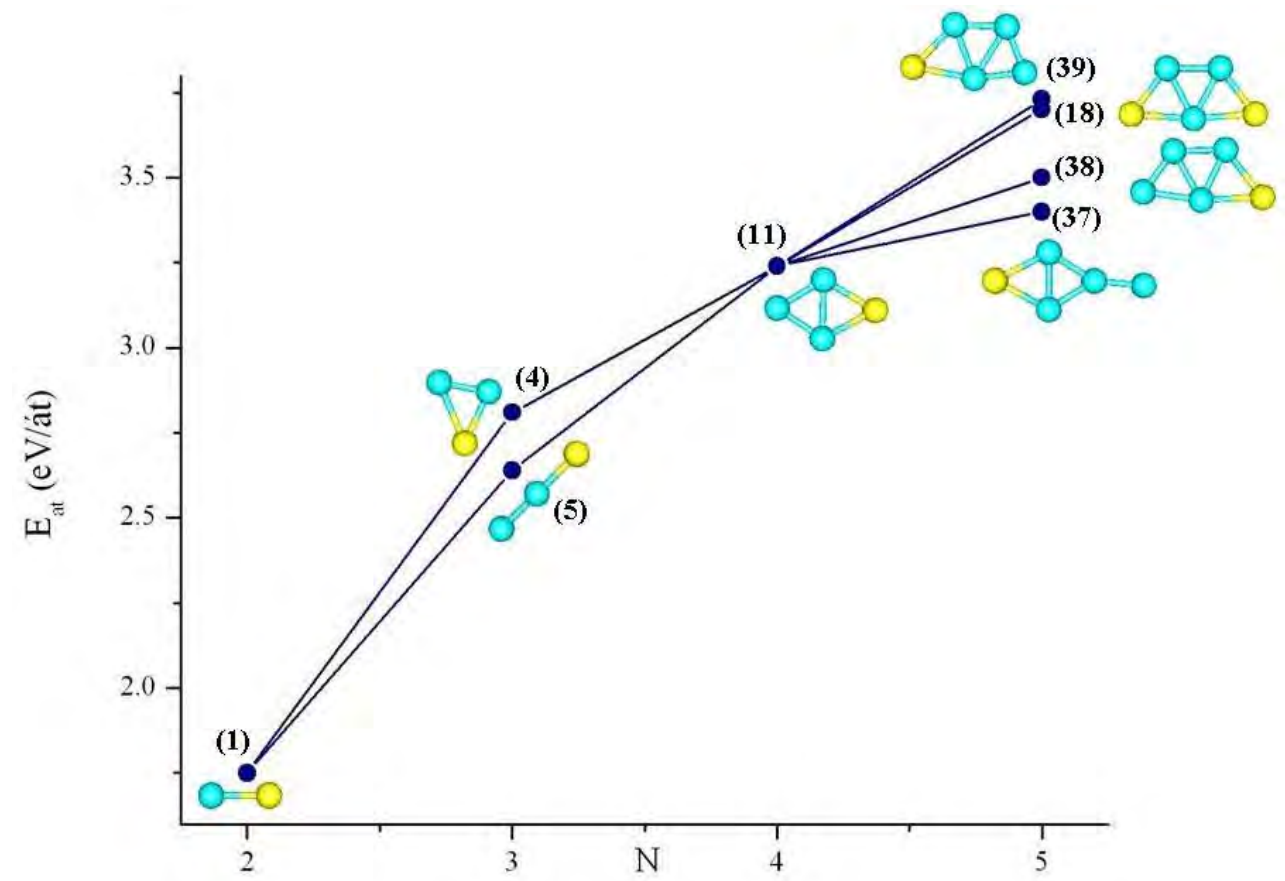

Figura 6.23. Patrón de crecimiento de los trímeros (4) y (5) y el tetrámero (11).

En la Figura 6.23 se muestra el camino de crecimiento del tetrámero (11), el cual puede provenir tanto del trímero (4) como del (5). El crecimiento de (4) se describe en el texto que acompaña a la Figura 6.22. Con respecto a la obtención de (5), se observa que un átomo de boro se une por medio de un ataque nucleofílico a B1 del dímero, formando un trímero lineal con dos uniones, menos favorable que formar (4), que posee tres uniones y geometría triangular (Tabla 6.7, Figuras 6.1 y 6.13). Asimismo, la obtención de (11) a partir de los trímeros resulta más favorable si ingresa un átomo de boro a un enlace B-P en (4), que si se une a un átomo terminal en (5) (Tabla 6.7, Figura 6.13).

Continuando el camino de crecimiento hacia la formación de los pentámeros, se observa que resulta de mayor energía de atomización el pentámero resultante de un ataque electrofílico de un átomo de boro, (39), que de un átomo de fósforo (18) (Tabla 6.8, Figura 6.14). Por otro lado, resulta considerablemente más estable el agregado de cinco átomos con estado electrónico doblete, (39), que el que posee estado electrónico cuatriplete, (38) (Tabla 6.5). Por otro lado, en este caso, la obtención de un pentámero con zonas susceptibles de ataque nucleofílico sobre átomos de fósforo y de ataque electrofílico sobre átomos de boro no resulta favorable, (37) (Tabla 6.9, Figura 6.17). Con respecto al pentámero más estable, (39), su formación se debe al ataque electrofílico de un átomo de boro en B1 de (11), zona de alta densidad de espín (Tabla 6.8, Figura 6.12).

Finalmente, los crecimientos más probables propuestos para cada trímero, desde el dímero BP, son:
$(1) \rightarrow(2) \rightarrow(7) \rightarrow(21)$
(Figura 6.19.b)
$(1) \rightarrow(3) \rightarrow(8) \rightarrow(18)$
(Figura 6.20)
(1) $\rightarrow$ (4) $\rightarrow$ (11) $\rightarrow$ (39)
(Figura 6.23)
$(1) \rightarrow(5) \rightarrow(11) \rightarrow(39)$
(Figura 6.23)

Donde los trímeros (4) y (5) llegan al mismo pentámero, por lo que llegarán al mismo hexámero, siendo éste crecimiento el más probable para todos los agregados. 


\subsection{Conclusiones}

Se estudiaron las geometrías, energías de atomización, frecuencias de vibración armónicas, potenciales electrostáticos moleculares, cargas ESP, densidades de espín y patrones de crecimiento de agregados de $\mathrm{B}$ y $\mathrm{P}$. Los parámetros geométricos y multiplicidades encontrados para el dímero y algunos de los trímeros y pentámeros están en acuerdo con lo informado en literatura, lo que indica que la metodología seguida para lograr estructuras estables es exitosa. Es importante destacar que en este trabajo se han encontrado confórmeros más estables que los reportados por otros autores.

El estado fundamental del dímero BP presenta estado electrónico triplete y una $\mathrm{E}_{\mathrm{at}}$ de 1.75 eV/át. El trímero de mayor estabilidad es BPB (4), de estado electrónico doblete, estructura triangular con dos átomos de boro y uno de fósforo y $\mathrm{E}_{\text {at }}$ de $2.81 \mathrm{eV} / \mathrm{a}$. Asimismo, todos los agregados de tres átomos poseen estado electrónico doblete. El tetrámero de mayor energía de atomización es BBBP (11), de estado electrónico triplete, estructura plana romboidal, con tres átomos de boro y uno de fósforo en su geometría. Con respecto a los pentámeros, BBPBB

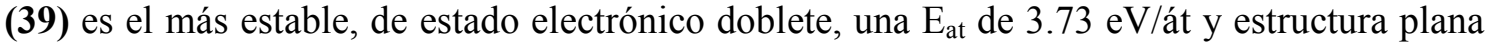
no lineal.

Analizando los modos vibracionales de todos los agregados, se encontró que los movimientos de wagging, rocking, scissoring y twisting se encuentran a frecuencias bajas; los estiramientos simétricos y asimétricos B-P y P-P a frecuencias intermedias; por último, los estiramientos simétricos y asimétricos P-P se encuentran a frecuencias altas, a más de $1031 \mathrm{~cm}^{-1}$.

Con excepción de BPP (2), los trímeros tienden a crecer hacia agregados planos no lineales, donde el patrón de crecimiento más probable es BP (1) $\rightarrow$ BPB (4) $\rightarrow$ BBBP (11) $\rightarrow$ BBPBB (39) y donde los agregados que se obtienen presentan $N-1$ átomos de boro. Los trímeros BPB (4) y BBP (5) crecen hacia el mismo tetrámero y pentámero. Tanto boro como fósforo no siempre se comportan como electrófilos o nucleófilos, ni atacan zonas con electrones desapareados. Las cargas ESP, los MEP y las densidades de espín son herramientas útiles para entender el comportamiento de agregados binarios. 


\section{Conclusiones generales}

En este trabajo se estudió el crecimiento de agregados pequeños III/V, de tipo $\mathrm{X}_{n} \mathrm{Y}_{m}, \mathrm{X}=\mathrm{B}$, Al; $\mathrm{Y}=\mathrm{N}, \mathrm{P} ; n=1-4$ y $m=1-4(n+m \leq 5)$. Se obtuvieron las geometrías, energías de atomización, frecuencias de vibración armónicas, potenciales electrostáticos moleculares, cargas ESP, densidades de espín y patrones de crecimiento de los clusters.

Todos los dímeros de partida, AlN, BN, AlP y BP, poseen estado electrónico triplete. El estado fundamental de los trímeros es doblete, siendo todos lineales, con excepción del trímero BPB, de estructura triangular. Por otro lado, se encuentra que resultan más estables los agregados de tres átomos que poseen sólo un átomo de aluminio, en el caso de agregados $\mathrm{Al} / \mathrm{N}$ y Al/P; y los agregados que contienen boro en su estructura, resultan más estables aquellos que incorporan sólo un átomo del grupo $\mathrm{V}$. Con respecto a los tetrámeros $\mathrm{Al} / \mathrm{N}$ y $\mathrm{B} / \mathrm{N}$, se obtuvo que son más estables los agregados lineales con tres átomos de nitrógeno en su estructura y de estado electrónico singlete; los tetrámeros $\mathrm{Al} / \mathrm{P}$ y $\mathrm{B} / \mathrm{P}$ resultaron ser de estructura romboidal. En los pentámeros III/V se encontraron diversas estructuras estables, siendo sólo el pentámero $\mathrm{Al} / \mathrm{N}$ de estado electrónico cuatriplete, $\mathrm{y}$ los otros de estado electrónico doblete.

Con respecto a los patrones de crecimiento, se obtuvo que la densidad de espín no es un condicionamiento para ningún crecimiento. Por otro lado, sí se encontró que las cargas ESP y potenciales electrostáticos moleculares ayudan a describir el comportamiento y el crecimiento de los agregados. Particularmente, en los agregados $\mathrm{B} / \mathrm{N}$, los trímeros crecen de manera de favorecer el ataque electrofílico con reordenamiento posterior de cargas. Este ataque se da por medio de un átomo de boro o un átomo de nitrógeno. La formación de enlaces de tipo $\mathrm{N}-\mathrm{N} \mathrm{a}$ medida que avanza el crecimiento, pareciera no ser determinante para la estabilidad de los agregados, obteniéndose una predominancia de uniones B-N, donde las diferencias de cargas sobre los átomos pueden deberse a las diferencias de electronegatividades entre $\mathrm{B}$ y N. Se espera que el crecimiento continúe de esta manera. Por el contrario, en los agregados de $\mathrm{Al} / \mathrm{N}$ se favorece, mediante ataques electrofílicos, la formación de uniones N-N sobre uniones Al-N y Al-Al. Se propone que el crecimiento continuará con la obtención de agregados con este tipo de uniones y cuyas cargas, y por consiguiente, su reactividad, sean mayores. Analizando los resultados de agregados de $\mathrm{B} / \mathrm{P}$ se observa que no existe siempre una tendencia a crecer mediante ataques electrofílicos, pero sí donde se formen enlaces B-B. El reordenamiento de carga se presenta de manera de obtener cargas de valor bajo, posiblemente debido a la similitud en los valores de las electronegatividades de B y P. Asimismo, para agregados de $\mathrm{Al} / \mathrm{P}$ tampoco se presenta la tendencia de crecer mediante ataques electrofílicos y cuando ello ocurre puede ser tanto Al o $\mathrm{P}$ quién se comporte como electrófilo. La poca diferencia en sus electronegatividades puede justificar que no existan siempre ataques electrofílicos en un crecimiento. Finalmente, es importante mencionar que esta metodología para la obtención de estructuras más allá del dímero permitió, en la mayoría de los casos, encontrar estructuras de mayor estabilidad que las reportadas en literatura. 


\section{APÉNDICE 1: Teoría del funcional de la densidad conceptual}

\section{A. Potencial químico electrónico y electronegatividad}

Parr y colaboradores ${ }^{93}$ se concentraron en la interpretación del potencial químico. Encontraron que $\mu$ puede ser escrito como la derivada parcial de la energía del sistema con respecto al número de electrones a un potencial externo fijo $v(r)$ :

$\mu=\left(\frac{\partial E}{\partial N}\right)_{v(r)}$

Para entender mejor su significado físico, se considera la variación en la energía de un sistema químico cuando pasa de un estado fundamental a otro estado $d E$ :

$d E=\left(\frac{\partial E}{\partial N}\right)_{v(r)} d N+\int\left(\frac{\partial E}{\partial v(r)}\right)_{N} \delta v(r) d r$

Por otro lado, $E$ es un funcional de $\rho(r)$,

$d E=\int\left(\frac{\delta E}{\delta \rho(r)}\right)_{v(r)} \delta \rho(r) d r$

$\mu$ puede ser escrito:

$\mu=\left(\frac{\delta E}{\delta \rho(r)}\right)_{v}$

Combinando A.2 y A.4,

$d E_{v}=\int \mu \delta \rho(r) d r=\mu \int \delta \rho(r) d r=\mu d N$

donde se indica explícitamente que la variación en la densidad electrónica $\rho(r)$ es para un dado potencial $v$. Por otro lado, a partir de teoría de perturbaciones, la corrección de primer orden a la energía del estado fundamental $d E^{(1)}$, debido a un cambio en el potencial externo, y a un número fijo de electrones es:

$d E^{(1)}=\int \Psi^{(0)} * \delta V \Psi^{(0)} d x^{N}=\int \rho(r) \delta V(r) d r$

$\Psi^{(0)}$ indica la función de onda no perturbada. Comparando A.6 con A.2,

$\rho(r)=\left(\frac{\delta E}{\delta v(r)}\right)_{N}$ 
Ahora ya se identificaron las dos primeras derivadas de $E$ con respecto a $N$ y $v$. Iczkowski y Margrave $^{94}$ demostraron, en base a energías de atomización y afinidades electrónicas experimentales, que la energía $E$ de un átomo puede representarse razonablemente bien por un polinomio de grado $n$ (número de electrones, $N$, menos la carga nuclear, $Z$ ), alrededor de $n=0$ :

$E=E(N)=a n^{4}+b n^{3}+c n^{2}+d n ; \quad n=N-Z$

A.8

Asumiendo que $E$ es continua y diferenciable ${ }^{95}$, se ve claramente que la pendiente a $n=0,(\partial E / \partial n)_{n=0}$ es una medida de la electronegatividad $\chi$. Iczkowski y Margrave propusieron definir a la electronegatividad como esta derivada, por lo tanto,

$\chi=-\left(\frac{\partial E}{\partial N}\right)$

para una carga nuclear fija. Debido a que los términos elevados a la tercera y cuarta potencia en la ecuación A.15 son despreciables, la definición de Mulliken ${ }^{96}$,

$\chi=\frac{1}{2}(I+A)$

donde I y A son la primera energía de ionización y la primera afinidad electrónica, respectivamente, fue visto como un caso particular de $\mathbf{A . 8}$, y fortaleció su propuesta.

Combinando las ecuaciones A.9, A.10 y A.4 y generalizando la restricción de carga nuclear fija a potencial externo fijo, $\mu$ se identifica, ahora, con el concepto de potencial químico introducido por Pauling en $1932^{97}$.

La energía de ionización y la afinidad electrónica pueden ser reemplazadas por las energías de los orbitales frontera HOMO (highest occupied molecular orbital) y LUMO (lowest unoccupied molecuar orbital), respectivamente, usando el teorema de Koopmans ${ }^{98}$,

$\chi=\frac{1}{2}\left(\varepsilon_{H O M O}+\varepsilon_{L U M O}\right)$

A.11

Se ha descripto una característica importante del desarrollo de la DFT moderna, la unión entre el concepto de potencial químico a la ecuación fundamental de la DFT, dando paso a la teoría del funcional de la densidad conceptual (conceptual DFT).

Kohn, Parr y Becke ${ }^{99}$ resaltaron que $\mu=(\partial E / \partial N)_{v}$ "contiene información considerable desde el punto de vista químico. $\mu$ caracteriza la tendencia de escape de los electrones del sistema en equilibrio. Los sistemas que reaccionan deben lograr, en el equilibrio, un potencial químico común. Este potencial químico es el negativo de la electronegatividad que proviene de la química estructural clásica."

Sin embargo, la relación A.4 fue muy criticada, entre otros autores, por Bader y colaboradores $^{100}$ en el supuesto que $N$ en un sistema mecano-cuántico cerrado es una propiedad continuamente variable del sistema. De todos modos, su uso es bastante natural en el contexto de átomos en moléculas en vez de átomos aislados. Estas "partes" pueden considerarse como sistemas abiertos que permiten la transferencia de electrones. Es más, su número de electrones no necesariamente cambia en valores enteros. 


\section{a) Esquema general: derivadas de la energía y funciones de respuesta en el ensamble canónico}

La introducción de la electronegatividad como un descriptor de reactividad remonta a la consideración de la respuesta de un sistema químico cuando es perturbado, a un cambio en su número de electrones a un potencial externo fijo. Inmediatamente demanda atención a su contraparte, $(\delta E / \delta v(r))_{N}$ (viene de la ecuación A.4), que a través de la expresión A.7, es claramente la función de densidad electrónica $\rho(r)$, indicando otra vez la importancia de la función de densidad electrónica.

Asumiendo más diferenciabilidad de $E$ con respecto a $N$ y $v(r)$, emerge una serie de funciones de respuesta, como se muestra en el siguiente esquema:

$$
\begin{array}{cc}
n=1 & \left(\frac{\partial E}{\partial N}\right)_{v(r)}=\mu=-\chi \\
\begin{array}{c}
\text { potencial químico electrónico } \\
\text { (-electronegatividad) }
\end{array} & \begin{array}{c}
\left(\frac{\partial E(r)}{\delta}\right)_{N}=\rho(r) \\
\text { densidad electrónica }
\end{array} \\
n=2 & \left(\frac{\partial^{2} E}{\partial N^{2}}\right)_{v(r)}=-\left(\frac{\partial \chi}{\partial N}\right)=\eta \\
\text { dureza química } & \left(\frac{\partial^{2} E}{\partial N \delta v}\right)=\left(\frac{\delta \mu}{\delta v(r)}\right)_{N}=\left(\frac{\partial \rho(r)}{\partial N}\right)_{v(r)}=f(r) \\
\text { función de Fukui electrónica } \\
\left(\frac{\delta^{2} E}{\delta v(r) \delta v\left(r^{\prime}\right)}\right)_{N}=\left(\frac{\delta \rho(r)}{\delta v\left(r^{\prime}\right)}\right)_{N}=\chi\left(r, r^{\prime}\right) \\
\text { función de respuesta lineal de dos variables }
\end{array}
$$

Emergen dos cantidades en las derivadas de primer orden: una cantidad global $\chi$, y una cantidad local $\rho(r)$, cuyo valor cambia punto a punto. La identificación del multiplicador de Lagrange con el negativo de la electronegatividad,

$$
\mu=-\chi=-\left(\frac{\partial E}{\partial N}\right)_{v}
$$

Ofrece una forma para calcular valores de electronegatividad para átomos, grupos funcionales, clusters y moléculas. Por otro lado, los resultados de la electronegatividad calculados mediante la ecuación A.12 corresponden a respuestas de la energía del sistema a perturbaciones electrofílicas $(d N<0)$ o nucleofílicas $(d N>0)$.

En las derivadas segundas, aparece por primera vez un kernel $\chi\left(r, r^{\prime}\right)$, representando la respuesta de una cantidad local en un punto dado $r$ a una perturbación a un punto $r^{\prime}$.

El concepto de dureza química y blandura química fue introducido por Pearson ${ }^{101}$ en conexión con el estudio de reacciones ácido-base de Lewis. Fue establecido que no había un orden simple de fuerzas de ácidos y bases que fuera válido para ordenar las fuerzas de interacción, como se miden con la entalpía de reacción, entre un ácido de Lewis y una base de 
Lewis. En este contexto, Pearson formuló un principio llamado HSAB (hard and soft acids and bases): ácidos duros interactúan preferiblemente con bases duras, y ácidos blandos con bases blandas. Sin embargo, la clasificación de un ácido o base nuevo no es una tarea fácil y la inserción de un compuesto en una escala de dureza o blandura es discutible. Un trabajo muy importante en el desarrollo de estos conceptos es el de Parr y Pearson ${ }^{102}$, en el cual se identifica a la dureza como la segunda derivada de la energía con respecto al número de electrones a un potencial externo fijo (ver esquema). Ofrece una definición precisa permitiendo el cálculo de esta cantidad y su confrontación con el experimento:

$\eta=\frac{1}{2}\left(\frac{\partial^{2} E}{\partial N^{2}}\right)_{v}$

Que también puede ser escrito:

$\eta=\frac{1}{2}\left(\frac{\partial \mu}{\partial N}\right)_{v}$

Mostrando que la dureza es la resistencia del potencial químico a cambios en el número de electrones.

Por otro lado, se estableció a la blandura como el recíproco de la dureza, mediante:

$$
S=\frac{1}{2 \eta}=\left(\frac{\partial N}{\partial \mu}\right)_{v(r)}
$$

Y, como para la electronegatividad, la dureza está relacionada con el gap de energía entre los orbitales frontera:

$\eta=\frac{1}{2}\left(\varepsilon_{L U M O}-\varepsilon_{\text {HOMO }}\right)$

A.16

La función electrónica de Fukui, presentada en el esquema, fue introducida por Parr y Yang ${ }^{103}$ como una generalización del concepto de Fukui de orbital molecular de frontera ${ }^{104}$, y juega un rol muy importante en conectar el principio HSAB con la teoría de orbital molecular de frontera. Esta función puede ser interpretada como el cambio en la densidad electrónica total $\rho(r)$ en cada punto $r$ cuando cambia el número total de electrones, o como la susceptibilidad a una perturbación externa del potencial químico del sistema a un punto $r$,

$$
f(r)=\left(\frac{\partial \rho(r)}{\partial N}\right)_{V}=\left(\frac{\delta \mu}{\delta v(r)}\right)_{N}
$$

Debido a la discontinuidad del potencial químico respecto a $N$, la derivada será diferente si se evalúa por la izquierda o por la derecha. Por lo tanto, se tienen tres funciones: $f^{+}(r)$ cuando la derivada se toma a medida que $N$ aumenta de $N$ a $N+\delta$; 
$f^{+}(r)=\left(\frac{\partial \rho(r)}{\partial N}\right)_{v}^{+}$

A.18

$f^{-}(r)$ cuando la derivada se evalúa a medida que $N$ disminuye de $N$ a $N-\delta$;

$f^{-}(r)=\left(\frac{\partial \rho(r)}{\partial N}\right)_{v}^{-}$

y $f^{0}(r)$ el promedio aritmético de las dos primeras,

$f^{0}(r)=\frac{1}{2}\left[f^{+}(r)+f^{-}(r)\right]$

A.20

La función $f^{+}(r)$ se asocia con el LUMO y mide la reactividad hacia un donor de electrones, la susceptibilidad a un ataque nucleofilico; la función $f^{-}(r)$ se asocia al HOMO y mide la reactividad hacia un agente aceptor, la susceptibilidad a un ataque electrofilico; y, finalmente $f^{0}(r)$, mide la reactividad hacia un radical.

$f^{+}(r) \approx\left[\rho_{N+1}(r)-\rho_{N}(r)\right] \approx \rho_{L U M O}(r)$

$f^{-}(r) \approx\left[\rho_{N}(r)-\rho_{N-1}(r)\right] \approx \rho_{\text {Номо }}(r)$

A.22

Por lo expresado anteriormente, el trabajo de Fukui propuso un índice de reactividad para interpretar la orientación en una reacción química y se volvió el ingrediente clave para el desarrollo de la teoría ${ }^{104}$

Por otro lado, la derivación usando la función de Fukui de Kohn-Sham también puede ser expresada en el contexto de la aproximación de orbital congelado (frozen orbital aproximation). Balawander y Komorowski ${ }^{105}$ derivaron y evaluaron, dentro del contexto de la teoría de Hartree-Fock, una expresión para los términos de relajación despreciados. Demostraron numéricamente que su contribución a la función de Fukui es pequeña. Es fácil demostrar que la función de Fukui puede ser escrita como:

$f_{k}^{+}=q_{k}(N+1)-q_{k}(N)$

A.23

$\mathrm{y}$

$f_{k}^{-}=q_{k}(N)-q_{k}(N-1)$

A.24

donde $q_{k}(N)$ es la carga de Mulliken en el átomo $k$-ésimo del sistema con $N$ electrones, $q_{k}(N+1)$ y $q_{k}(N-1)$ son las cargas del átomo $k$-ésimo de la molécula con $(N+1)$ y $(N-1)$ electrones en una aproximación de core congelado (frozen core approximation).

Una característica de la función de Fukui a tener en cuenta en el momento del cálculo es su signo, ya que un valor negativo de la misma, como está definida en las expresiones A.23 y A.24, implicaría situaciones sin sentido físico ${ }^{106}$ para el estado fundamental de moléculas en su geometría de equilibrio: 
- $\quad$ Si para el átomo $\mathrm{A}$ en la molécula $f_{A}^{+}>0$ y $f_{A}^{-}<0$, significaría (usando las expresiones A.23 y A.24) que

$$
q_{A}(N+1)-2 q_{A}(N)+q_{A}(N-1)>0
$$

La función $q(N)$ sería convexa, que es un resultado sin sentido físico, dado que la evidencia experimental dice que la capacidad de carga de un átomo en una molécula se reduce cuanto más cargada está la molécula.

- $\quad$ Si para el átomo A en la molécula $f_{A}^{+}<0$ y $f_{A}^{-}<0$, significaría que

$$
q_{A}(N+1)-q_{A}(N-1)<0
$$

que tampoco tiene significado físico.

- La última posibilidad, $f_{A}^{-}>0$ y $f_{A}^{+}>0$, es el único caso que no implica un significado no físico. Por lo tanto, se puede decir que para el estado fundamental de un sistema químico en su geometría de equilibrio, una función de Fukui negativa sólo puede ocurrir para el indice $f_{A}^{+14}$.

\section{B. Función de localización electrónica (ELF, electron localization function)}

La función de localización electrónica, ELF, ayuda a entender el concepto empírico de localización electrónica, especialmente la localización del par electrónico dentro del espíritu de las estructuras de Lewis. Fue introducida por Becke y Edgecombe ${ }^{107}$ y aplicada con posterioridad por Savin y coautores ${ }^{108}$ a un gran rango de sistemas, desde átomos a moléculas y sólidos. Algunos años después, Silvi y Savin ${ }^{109}$ propusieron una clasificación topológica de ELF que ayuda a dar cuantificación a los procesos químicos asociados a la función. Luego de eso, y con el lanzamiento del programa gratuito TOPMOD por Silvi y colaboradores ${ }^{110}$, ha habido muchas más aplicaciones de la ELF a moléculas, clusters y sólidos.

Se puede calcular la ELF usando el software TOPMOD. Usa las salidas de programas populares de cálculos de estructura electrónica como GAUSSIAN y GAMESS para obtener la ELF y elaborar el análisis topológico necesario.

\section{a) Propuesta de Becke}

Al estudiar la correlación entre electrones fue muy claro que, debido al principio de exclusión de Pauli, el movimiento de electrones del mismo espín está más correlacionado que el movimiento entre electrones de espines diferentes. Por lo tanto, parece conveniente estudiar la densidad electrónica de pares para electrones del mismo espín y para electrones de distinto espín, separadamente. La densidad electrónica de pares, $\rho_{2}^{\sigma \sigma^{\prime}}\left(r_{1}, r_{2}\right)$, da la probabilidad de encontrar un electrón de espín $\sigma$ en el punto $r_{1}$ cuando un segundo electrón de espín $\sigma^{\prime}$ está localizado en el punto $r_{2}$. Debido a que la interacción interelectrónica depende sólo de la distancia entre los electrones y no de la orientación angular, es conveniente cambiar el sistema 
de coordenadas al definido por: $r=\frac{1}{2}\left(r_{1}+r_{2}\right)$ y $s=r_{1}-r_{2}$. Entonces, sólo es necesario el promedio esférico de la densidad electrónica de pares, que se define:

$\rho_{2}^{\sigma \sigma^{\prime}}\left(r_{1}, s\right)=\frac{1}{4 \pi} \int \rho_{2}^{\sigma \sigma^{\prime}}\left(r_{1}, s\right) d \Omega_{s}$

B.1

donde la integración es sobre los ángulos del vector $s$. La ventaja del nuevo sistema de coordenadas es que ahora la interacción electrónica no depende de seis variables $\left(r_{1} \mathrm{y} r_{2}\right)$, sino sólo de cuatro $(r$ y $s$ ).

Luego, Becke y Edegecombe ${ }^{111}$ prefirieron trabajar con la densidad de pares condicional para electrones del mismo espín que es una medida de la probabilidad de encontrar un electrón en $r_{2}$ cuando con certeza hay un electrón del mismo espín en la posición $r_{1}$. Está dada por,

$P^{\sigma \sigma}(r, s)=\rho_{2}^{\sigma \sigma^{\prime}}(r, s) / \rho_{\sigma}(r)$

B.2

donde $\rho_{\sigma}(r)$ es la densidad electrónica de electrones con espín $\sigma$ que dentro de la aproximación de Kohn-Sham

$\rho_{\sigma}(r)=\sum_{i}^{\sigma} \phi_{i}(r)^{2}$

con las funciones auxiliares de Kohn-Sham dadas por el conjunto $\left\{\phi_{i}\right\}$ y la suma es sobre todos los orbitales ocupados de espín $\sigma$.

Luego, propusieron examinar la expansión en series de Taylor del promedio esférico de la densidad de pares condicional en las cercanías del punto $s=0$ donde se mide el comportamiento de corto alcance del electrón en el punto $r_{2}$, acercándose al punto de referencia $r_{1}$. El término principal de la serie está dado por,

$P^{\sigma \sigma}(r, s)=\frac{1}{3}\left(\tau_{\sigma}-\frac{1}{4} \frac{\left(\nabla \rho_{\sigma}\right)^{2}}{\rho_{\sigma}} s^{2}\right)+\ldots$

donde $\tau_{\sigma}$ es la densidad de energía cinética definida positiva por,

$\tau_{\sigma}=\sum_{i}^{\sigma}\left(\nabla \phi_{i}\right)^{2}$

B.5

La expansión de Taylor contiene toda la información de la localización electrónica. Cuanto más pequeña sea la probabilidad de encontrar el segundo electrón cerca del punto $r$, más localizado estará el electrón de referencia. Por lo tanto, la localización electrónica está directamente relacionada al paréntesis en el lado derecho de la ecuación B.4,

$D_{\sigma}=\tau_{\sigma}-\frac{1}{4} \frac{\left(\nabla \rho_{\sigma}\right)^{2}}{\rho_{\sigma}} s^{2}$

B.6 
que es una cantidad no negativa ${ }^{112}$. También se puede demostrar fácilmente que $D_{\sigma}$ desaparece para el átomo de hidrógeno y también para el helio en la aproximación de HartreeFock y también se espera que sea despreciable en las regiones cercanas al núcleo, donde se encuentra los electrones más localizados.

Por consiguiente, es razonable esperar que la cantidad $D_{\sigma}$ sea pequeña en las regiones del espacio donde la probabilidad de encontrar un electrón localizado o un par de electrones localizados es alta. Entonces, Becke y Edgecombe propusieron dos reglas de escalamiento adicionales. El gas electrónico homogéneo, cuya densidad de energía cinética está dada por $D_{\sigma}^{0}=c_{f} \rho^{5 / 3}$, se usa como referencia, y por conveniencia numérica la función se mapea a una que se define entre cero y uno. Por lo que ellos propusieron la siguiente función de localización electrónica:

$E L F=\left(1+\chi_{\sigma}^{2}\right)^{-1}$

donde

$\chi_{\sigma}=D_{\sigma} / D_{\sigma}^{0}$

B.8

De esta manera, la siguiente desigualdad se obedece:

$0 \leq E L F \leq 1$

B.9

donde un valor de ELF cercano a uno corresponde a una región del espacio donde hay una alta probabilidad de encontrar localización electrónica, mientras que un valor de ELF cercano a un medio corresponde a un comportamiento como el del gas electrónico. La función así definida es independiente de cualquier transformación unitaria de los orbitales y, en principio, es derivable a partir de la densidad electrónica. De hecho, es sólo necesario tener algunos de los procedimientos actualmente usados para obtener el potencial de Kohn-Sham a partir de la densidad; para obtener a partir del potencial, por medio de las ecuaciones de Kohn-Sham, los orbitales necesarios para calcular la función $\tau_{\sigma}$ como lo hicieron Kohout y Savin ${ }^{113}$. También, la función puede ser calculada a partir de la densidad obtenida experimentalmente.

\section{b) La interpretación de Savin}

La ELF está mayormente basada en una interpretación de la densidad de probabilidad de pares condicional para electrones del mismo espín. Una interpretación diferente fue dada por Savin y colaboradores, quienes tuvieron en cuenta que el término $D_{\sigma}$ puede generalizarse para cualquier densidad $\rho$ independiente del espín, como:

$D=\frac{1}{2} \sum_{i}\left(\nabla \phi_{i}\right)^{2}-\frac{1}{8} \frac{(\nabla \rho)^{2}}{\rho}$

Se puede verificar fácilmente que, para sistemas cerrados, ambas expresiones, B.6 y B.10, son iguales. El segundo término del lado derecho de la última expresión es reconocido como la densidad de energía cinética de von Weizsacker ${ }^{114}$, la cual es exacta para el átomo de hidrógeno, el de helio y para un sistema de bosones. Mientras que el primer término 
representa la densidad de energía cinética de la molécula en estudio. Por lo tanto, en la región del espacio donde hay una probabilidad alta de encontrar un par de electrones localizados la densidad de energía cinética de von Weizsacker será un buen modelo y la función $D$ tendrá un valor cercano a cero. Dentro de esta interpretación la ELF es formalmente una medida del exceso de energía cinética debido al principio de exclusión de Pauli. Ahora, debería ser claro que el concepto de localización de par electrónico no es más que una manifestación del principio de exclusión de Pauli.

\section{c) Estabilidad numérica}

Una característica importante de la ELF es su estabilidad numérica con respecto al nivel de teoría utilizado para obtener la densidad electrónica. Al contrario de otros análisis de población que dependen seriamente del conjunto de bases usado o el método de cálculo, la ELF es bastante independiente de estos cambios. Esta propiedad de la ELF puede explicarse viendo el comportamiento cualitativo de la densidad electrónica total, que también es bastante independiente del nivel de cálculo. Esto significa que, topológicamente, la función es invariante al nivel de cálculo ${ }^{115}$.

\section{d) Cómo se evalúa la ELF}

Como se ha dicho anteriormente, ELF mide el exceso de densidad de energía cinética debido a la repulsión de Pauli. En la región del espacio donde la repulsión de Pauli es fuerte (electrones solos o comportamiento de pares de espín opuestos), ELF se acerca a 1; mientras que donde la probabilidad de encontrar electrones de mismo espín juntos es alta, ELF tiende a 0 .

ELF permite dividir el espacio en basins de atractores donde los pares electrónicos están localizados. Estos basins pueden ser basins internos rodeando un núcleo, o basins de valencia, que no incluyen un núcleo. El número de conexiones de un dado basin de valencia se llama orden sináptico. Un basin de valencia disináptico corresponde a una unión de dos centros, mientras que uno monosináptico caracteriza un par libre. Las uniones multicéntricas, tales como uniones de tres centros y dos electrones (3c-2e), se llaman basins polisinápticos.

\section{C. Átomos en moléculas}

Tal vez la manera más rigurosa de dividir el volumen molecular en subespacios atómicos es el método Átomos en Moléculas de Bader ${ }^{116}$. La densidad electrónica es el cuadrado de la función de onda integrada sobre $N-1$ coordenadas (no importa qué coordenadas, dado que todos los electrones son idénticos),

$$
\rho\left(r_{1}\right)=\int\left|\Psi\left(r_{2}, r_{3}, \ldots, r_{N}\right)\right|^{2} d r_{2} d r_{3} \ldots d r_{N} \quad \text { C.1 }
$$

La densidad electrónica es una función de tres coordenadas espaciales, y puede ser analizada en términos de su topología (máximos, mínimos y puntos de ensilladura). En la gran mayoría de los casos los máximos de la densidad electrónica ocurren en el núcleo (o muy cerca de él), que actúa como atractor de la densidad electrónica. En cada punto del espacio el gradiente de la densidad electrónica apunta en la dirección del atractor (local) más fuerte. Esto forma una 
manera rigurosa de dividir el espacio físico en subespacios atómicos: empezando en un cierto punto en el espacio una serie de pasos infinitesimales pueden ser tomados en la dirección del gradiente hasta que se encuentra al atractor. El conjunto de dichos puntos forman el basin atómico asociado con el atractor (núcleo).

La frontera entre dos basins atómicos tridimensionales es una superficie bidimensional. Los puntos en dicha superficie divisora tienen la propiedad de que el gradiente de la densidad electrónica es perpendicular al vector normal de la superficie; es decir, la parte radial de la derivada de la densidad electrónica es cero (el flujo electrónico es cero).

Una vez que el volumen molecular ha sido dividido, la densidad electrónica puede ser integrada dentro de cada basin atómico para dar una carga atómica neta. Como la superficie divisora está definida rigurosamente en términos de la función de onda, las cargas convergirán a valores específicos a medida que aumenta la calidad de la función de onda. Además, como sólo la densidad electrónica está involucrada, los resultados son bastante insensibles al nivel teórico usado para generar la función de onda. Análogamente al procedimiento de Mulliken, las cargas resultantes no reproducirán los momentos dipolares, quadrupolares, etc.; tampoco conducirán a representaciones particularmente buenas del potencial electrostático. A pesar de lo estéticamente atractivo de derivar cargas en términos de basins atómicos, no son apropiados para la modelización.

Hay dos problemas más con las cargas atómicas de AIM. Uno es la magnitud de las cargas calculadas para uniones polares, la redistribución interna es a menudo significativamente mayor a los valores "comúnmente aceptados"117. El otro problema en el enfoque AIM es la presencia de atractores no nucleares en ciertos sistemas metálicos como clusters de litio y sodio $^{118}$. Mientras que estos sistemas son interesantes por ellos mismos, arruinan la idea de los electrones asociados con núcleos formando átomos dentro de moléculas.

La división del volumen molecular en basins atómicos viene de un análisis más profundo basado en el principio de acción estacionaria. La forma de los basins atómicos y las densidades electrónicas asociadas en un grupo funcional son muy similares en moléculas diferentes. Las propiedades locales de la función de onda son, entonces, transferibles, en una muy buena aproximación, que racionaliza las bases de la química orgánica, que los grupos funcionales reaccionan similarmente en moléculas diferentes. Puede demostrarse que cualquier propiedad observable puede ser escrita como la suma correspondiente de contribuciones atómicas:

$$
\langle A\rangle=\sum_{i}^{\substack{b a \sin s \\ \text { atómi cos }}}\langle A\rangle_{i}
$$

La energía total, por ejemplo, puede ser escrita como la suma de energías atómicas, y estas energías atómicas son también casi constantes para las mismas unidades estructurales en diferentes moléculas. Los basins atómicos son probablemente la analogía químico-cuántica más cercana a los conceptos químicos de átomos dentro de una molécula.

La segunda derivada de la densidad electrónica, la matriz hessiana, es una función de tres coordenadas cartesianas, es decir, es una matriz de $3 \times 3$. En los puntos estacionarios, puede ser diagonalizada y se puede determinar el número de autovalores negativos. El basin atractor es un máximo en su totalidad, tiene tres autovalores negativos. Se encuentran usualmente otros puntos estacionarios entre núcleos que están unidos. Dichos puntos tienen un mínimo en la densidad electrónica en la dirección del núcleo, y un máximo en direcciones perpendiculares, es decir, hay un autovalor positivo y dos negativos en el Hessiano. Estos puntos se conocen como puntos críticos de unión. 
Como se mencionó con anterioridad, los puntos donde el gradiente de la densidad electrónica $\nabla \rho(r)$, se anula están caracterizados por los tres autovalores $\left(\lambda_{1}, \lambda_{2}, \lambda_{3}\right)$ de la matriz hessiana de $\rho(r)$. Varias propiedades que pueden ser evaluadas en un punto crítico de enlace constituyen herramientas muy poderosas para clasificar las interacciones entre dos fragmentos. Si la carga electrónica está localmente concentrada en la región del punto crítico de enlace esto conduce a una interacción encontrada típicamente en uniones covalentes polarizadas. Estas interacciones están caracterizadas por grandes valores de $\rho(r), \nabla \rho(r)<0$, | $\lambda_{1} / / \lambda_{3}>1$ y G/ $\rho(r)>1$, donde $\mathrm{G}$ es la densidad de energía cinética local en el punto crítico de enlace.

Por otro lado, si la densidad electrónica está concentrada localmente en cada sitio atómico, la interacción es ahora de capa cerrada y tiene características de uniones altamente iónicas, uniones hidrógeno e interacciones de van der Walls y está caracterizada por valores pequeños de $\rho(r), \nabla \rho(r)>0,\left|\lambda_{1}\right| / \lambda_{3}<1$ y $\mathrm{G} / \rho(r)>1$.

Finalmente, la elipticidad, $\varepsilon$, indica la desviación de la densidad de carga electrónica de la simetría de una unión química. Grandes valores de $\varepsilon$ indican que el enlace en estudio tiene una inestabilidad intrínseca y tenderá a distorsionarse para relajarse a una forma más estable. Se muestra un resumen de los parámetros en la Tabla C.1.

Tabla C.1 Propiedades de los puntos críticos.

\begin{tabular}{c|c} 
Interacciones covalentes & Interacciones de capa cerrada \\
\hline grandes valores de $\rho(r)$ & pequeños valores de $\rho(r)$ \\
\hline$\nabla \rho(r)<0$ & $\nabla \rho(r)>0$ \\
\hline$\left|\lambda_{1}\right| / \lambda_{3}>1$ & $\left|\lambda_{1}\right| / \lambda_{3}<1$ \\
\hline $\mathrm{G} / \rho(r)<1$ & $\mathrm{G} / \rho(r)>1$
\end{tabular}

\section{Densidad de estados (DOS, density of states)}

La densidad de estados, $\rho_{D O S}$, indica el número de estados por unidad de volumen que se encuentran en un intervalo de energía entre $E y d E$. El número total de estados por unidad de volumen, $N$, se obtiene integrando desde $-\infty$ hasta la energía de Fermi,

$$
N=\int_{-\infty}^{E_{\text {fermi }}} \rho_{D O S}(E) d E \quad \text { D.1 }
$$

Asumiendo que cada estado es ocupado por un electrón, el resultado de la integración es igual al número total de electrones. Por lo tanto, las gráficas de DOS muestran la distribución de los electrones en función de la energía.

\section{E. Crystal orbital overlap population (COOP)}

El índice $\mathrm{COOP}^{119}$ fue propuesto para encontrar uniones en sólidos y superficies. Tiene su base en el análisis poblacional de Mulliken. Se toma, como punto de partida, un orbital de dos centros:

$\psi=c_{1} \phi_{1}+c_{2} \phi_{2}$

E.1 
Se requiere, además, que $\psi$ esté normalizada:

$$
\int|\psi|^{2} d \tau=1=\int\left|c_{1} \phi_{1}+c_{2} \phi_{2}\right|^{2} d \tau=c_{1}^{2}+c_{2}^{2}+2 c_{1} c_{2} S_{12} \quad \text { E.2 }
$$

El término $2 \mathrm{c}_{1} c_{2} S_{12}$ se denomina población de solapamiento y es una característica de la unión entre los dos centros. Cuando la población de solapamiento entre los centros involucrados se suma sobre todos los orbitales moleculares ocupados del sistema, se tiene una medida cuantitativa del tipo de unión entre aquellos. Así, una población de solapamiento positiva indica una interacción enlazante, mientras que una negativa representa una interacción antienlazante entre los átomos. De esta manera, se construye fácilmente un indicador de enlace para los átomos involucrados.

Es posible también calcular la población de solapamiento entre dos átomos como una función de la energía de los orbitales (estados) del sistema, obteniendo una evolución de las características del solapamiento con el aumento de energía. A esta nueva función se la denomina Crystal Orbital Overlap Population, ya que su origen se remonta al estudio de propiedades de enlace en sólidos cristalinos, y representa la variación de la población de solapamiento entre dos átomos en función de la energía, ponderada por la densidad de estados.

Las características generales de las gráficas de COOP son que las zonas positivas son regiones de enlace y las negativas de antienlace. La amplitud de la curva depende del número de estados en ese intervalo de energía, la magnitud del solapamiento y el tamaño y los coeficientes de los orbitales moleculares. La integración de la curva de COOP hasta el nivel de Fermi es la población de solapamiento total de la unión en cuestión. 


\section{APÉNDICE 2: Análisis preliminares de agregados de Al/N}

En la primera etapa de trabajo se optimizaron todas las posibilidades de agregados $\mathrm{Al} / \mathrm{N}$ de seis átomos, a nivel B3LYP/6-311+g(3df), luego se optimizaron dímeros, trímeros, tetrámeros y pentámeros de los agregados, con el mismo nivel de teoría. Se calcularon poblaciones de Mulliken, gráficos de COOP (crystal orbital overlap population) y de densidad de estados (DOS, density of states). También se obtuvieron índices de DFT conceptual ${ }^{120}$ (funciones de Fukui, dureza, potenciales de ionización y afinidades electrónicas). Remitirse al apéndice 1, para más detalles sobre el fundamento teórico de los índices de DFT conceptual.

\section{A. Optimización de geometrías}

Se obtuvieron las geometrías de equilibrio, energías de atomización y multiplicidades de 11 hexámeros (uno de ellos hexagonal alternado), 5 trímeros (4 lineales y 1 no lineal), 6 tetrámeros ( 3 lineales, 2 no lineales y un rombo alternado) y 5 pentámeros ( 2 lineales y 3 no lineales); optimizándose la multiplicidad menor posible y la siguiente. Los cálculos se realizaron sin restricciones de simetría. Las geometrías de equilibrio obtenidas se muestran en las Figuras A.1, A.2 y A.3.

El análisis de los hexámeros se realizó con fines comparativos respecto a resultados ya reportados en la literatura ${ }^{67}$.

Las Tablas A.1, A.2 y A.3 muestran las energías de atomización y multiplicidades de los agregados más estables. 


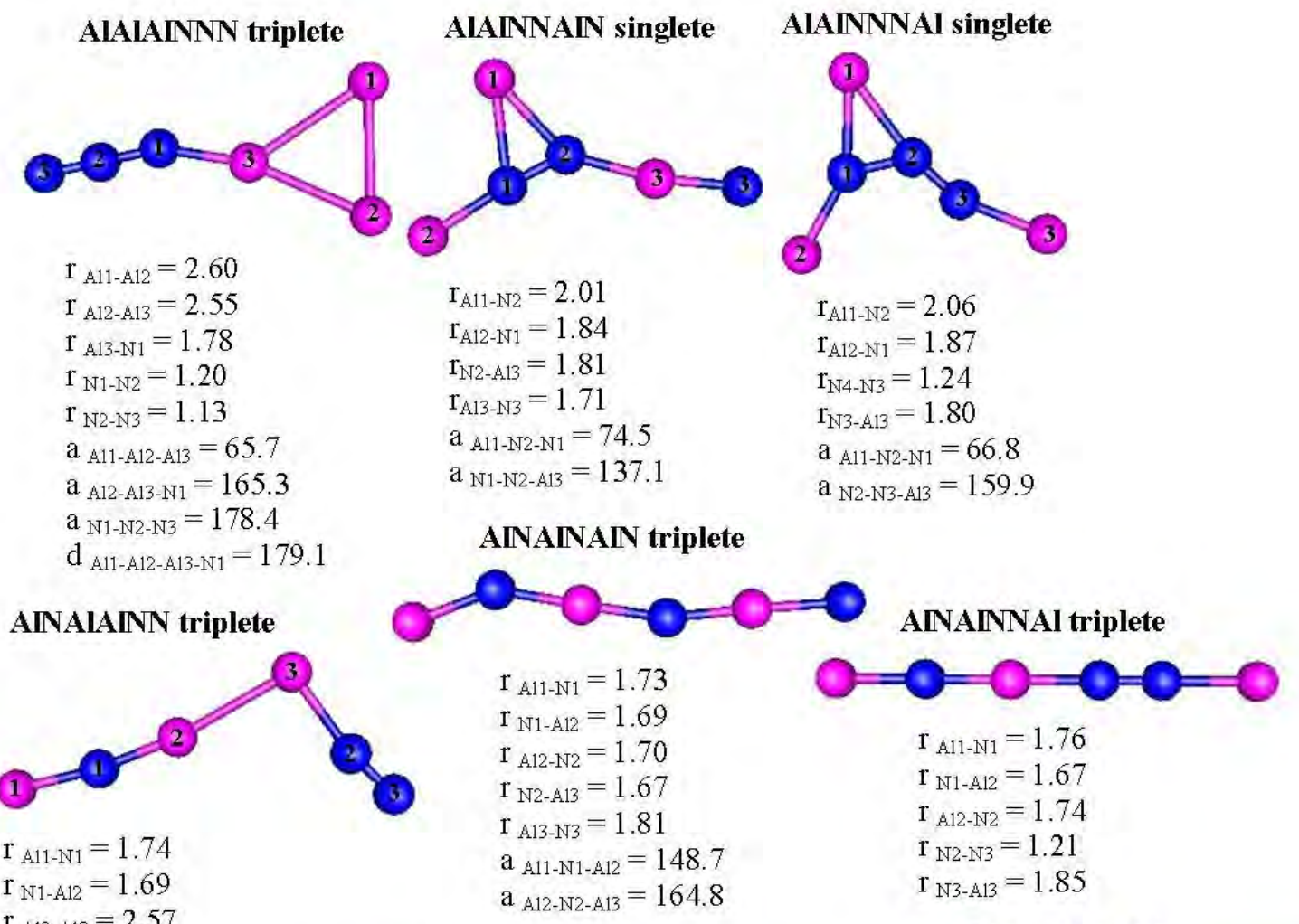

$\mathrm{r}_{\mathrm{A} 12-\mathrm{A} 13}=2.57$

$\mathrm{r}_{\mathrm{A} 13-\mathrm{N} 2}=1.95$

$\mathrm{r}_{\mathrm{N} 2-\mathrm{N} 3}=1.12$

$\mathrm{a}_{\mathrm{N} 1-\mathrm{A} 12-\mathrm{A} 13}=172.2$

$a_{\text {A12-A13-N2 }}=96.1$

$\mathrm{a}_{\mathrm{A} 13-\mathrm{N} 2-\mathrm{N} 3}=169.5$

$\mathrm{d}_{\mathrm{A} 12-\mathrm{A} 13-\mathrm{N} 2 \mathrm{~N} \mathrm{~N} 3}=180.0$

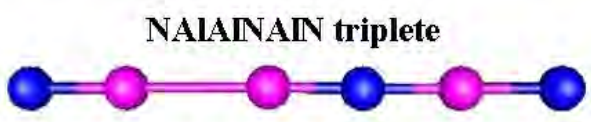

NAINNNAI triplete

AINNAIAIN triplete

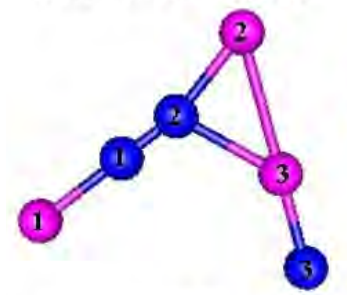

$\mathrm{r}_{\mathrm{N} 1-\mathrm{A} 11}=1.73$

$\mathrm{r}_{\mathrm{A} 11-\mathrm{A} 12}=1.74$

$\mathrm{r}_{\mathrm{A} 12 \mathrm{-N} 2}=1.66$

$\mathrm{r}_{\mathrm{N} 3-\mathrm{Al}}=1.72$

$\mathrm{r}_{\mathrm{A} 13-\mathrm{N} 3}=1.76$

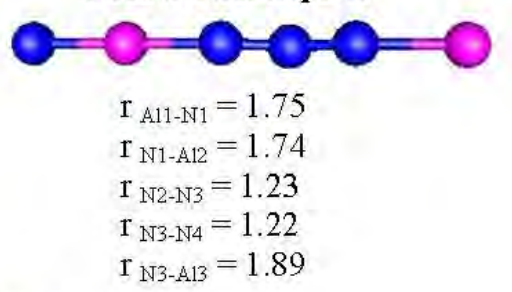

AINAINAIN singlete

$\mathrm{r}_{\mathrm{A} 11-\mathrm{N} 1}=1.85$

$\mathrm{r}_{\mathrm{N} 1 \mathrm{-N} 2}=1.22$

$\mathrm{r}_{\mathrm{N} 2-\mathrm{A} 12}=1.85$

$\mathrm{r}_{\mathrm{A} 12-\mathrm{A} 13}=2.58$

$\mathrm{r}_{\mathrm{A} 13-\mathrm{N} 3}=1.76$

AINNNAIN singlete

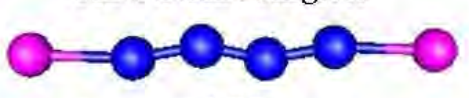

$\mathrm{r}_{\mathrm{A} 11-\mathrm{N} 2}=1.80$

$\mathrm{r}_{\mathrm{N1} 1 \mathrm{-N} 2}=\mathrm{r}_{\mathrm{NN} 3-\mathrm{N} 4}=1.23$

$\mathrm{r}_{\mathrm{N} 2-\mathrm{N} 3}=1.16$

$\mathrm{r}_{\mathrm{N} 4-\mathrm{A} 12}=1.76$

$\mathrm{a}_{\mathrm{N1} 1-\mathrm{N} 2-\mathrm{N} 3}=158.1$

$a_{\mathrm{N} 3-\mathrm{N} 4-\mathrm{A} 22}=167.6$

$\mathrm{d}_{\text {A.11-N1-N2-N3 }}=-179.1$

$\mathrm{d}_{\mathrm{N} 2-\mathrm{N} 3-\mathrm{N} 4-\mathrm{A} 2 \mathrm{2}}=-179.9$

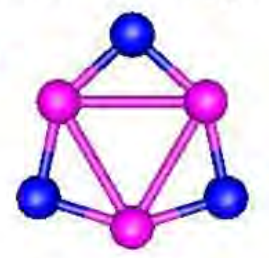

$\mathrm{r}_{\mathrm{AL}-\mathrm{N}}=1.75$

$\mathrm{a}_{\mathrm{Al}-\mathrm{N}-\mathrm{A} 1 \mathrm{l}}=95.3$

$\mathrm{a}_{\mathrm{N}-\mathrm{Al}-\mathrm{N}}=144.7$

$a_{12-A 12-A 13}=$

Figura A.1. Geometrías de agregados de hasta 6 átomos. Las esferas azules corresponden a átomos de nitrógeno, y las rosas a átomos de aluminio. Las distancias están en $\AA$, los ángulos en grados. Los átomos se numeran de izquierda a derecha, o como lo muestra la Figura. 


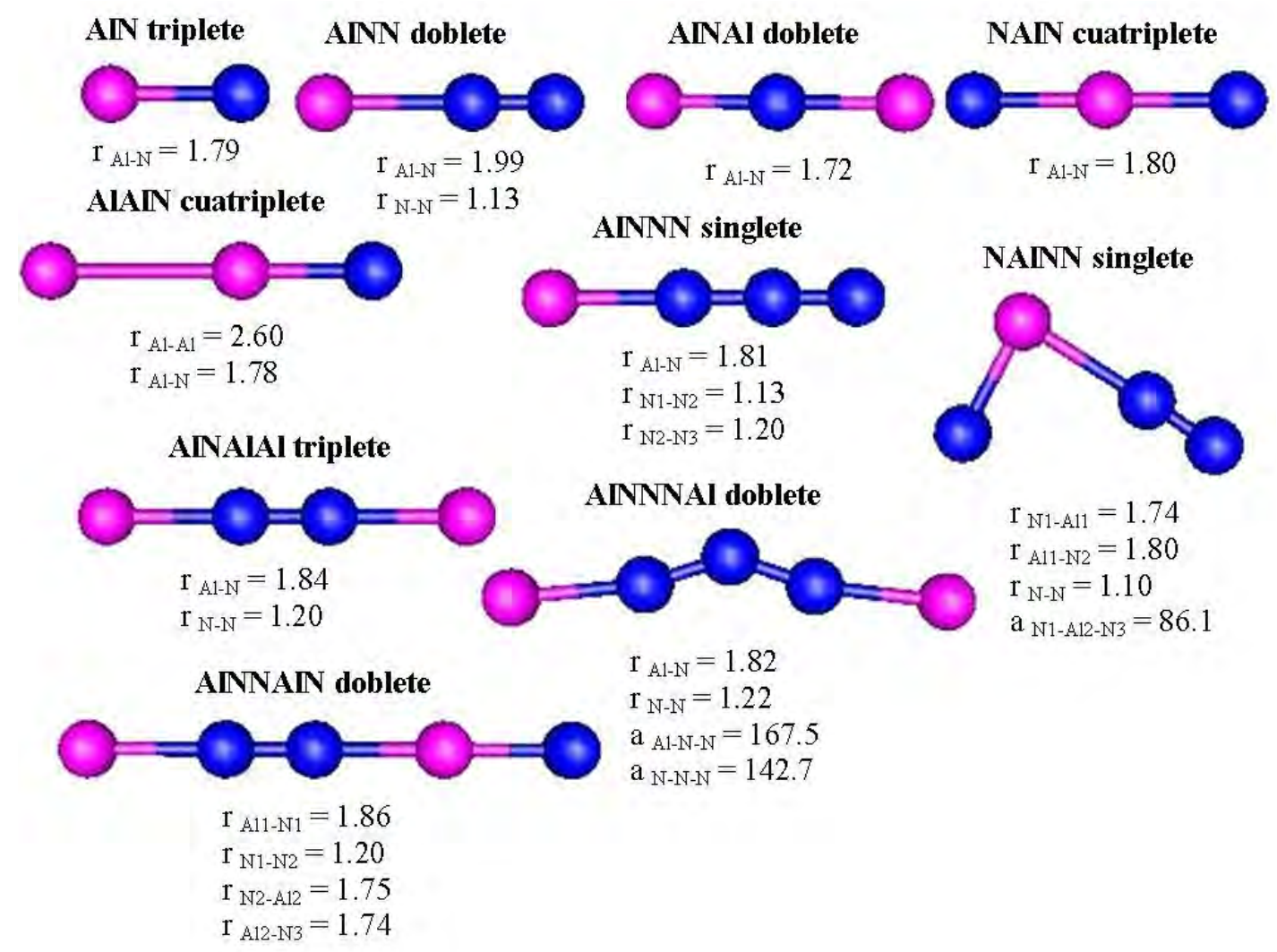

Figura A.2. Geometrías de agregados lineales. Las esferas azules corresponden a átomos de nitrógeno, y las rosas a átomos de aluminio. Las distancias están en Å, los ángulos en grados. Los ángulos planos (a) que no se especifican son $180.0^{\circ}$ y los diedros (d) $0.0^{\circ}$. Los átomos se numeran de izquierda a derecha, o como lo muestra la Figura. 


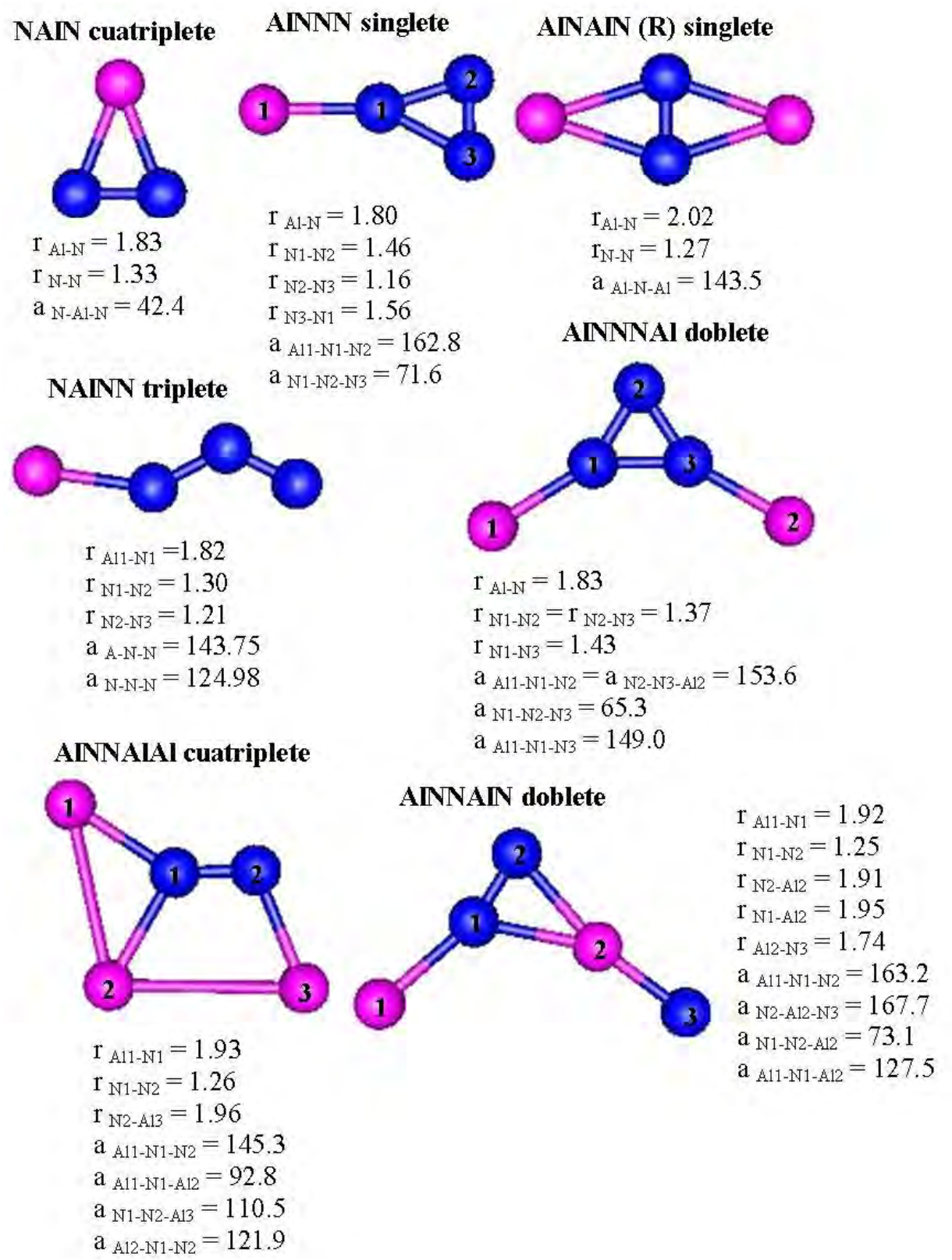

Figura A.3. Geometrías de agregados no lineales. Las esferas azules corresponden a átomos de nitrógeno, y las rosas a átomos de aluminio. Las distancias están en $\AA$, los ángulos en grados. Los ángulos planos (a) que no se especifican son $180.0^{\circ}$ y los diedros (d) $0.0^{\circ}$. Los átomos se numeran de izquierda a derecha, o como lo muestra la Figura. 
Tabla A.1. Multiplicidades de espín $\left(\mathrm{M}_{\mathrm{S}}\right)$ y energías de atomización $\left(\mathrm{E}_{\mathrm{at}}\right)$ en eV/át, de los hexámeros. $(\mathrm{H})$ significa hexagonal.

\begin{tabular}{l|c|c}
\multicolumn{1}{c|}{ Agregado } & $\mathbf{M}_{\mathbf{s}}$ & $\mathbf{E}_{\mathbf{a t}}$ \\
\hline AlAlAlNNN & 3 & 2.90 \\
\hline AlAlNNAlN & 1 & 2.51 \\
\hline AlAlNNNAl & 1 & 3.08 \\
\hline AlNAlAlNN & 3 & 3.12 \\
\hline AlNAlNAlN & 3 & 2.85 \\
\hline AlNAlNNAl & 3 & 3.11 \\
\hline AlNNAlAlN & 3 & 2.61 \\
\hline NAlAlNAlN & 3 & 2.27 \\
\hline NAlNNNAl & 3 & 2.88 \\
\hline AlNNNAlN & 1 & 3.17 \\
\hline AlNAlNAlN (H) & 1 & 3.16
\end{tabular}

Se observa en la Tabla A.1 que el agregado más estable de seis átomos es el hexámero AlNNNAIN, de geometría inicial lineal, con una energía de atomización de $3.17 \mathrm{eV} / a ́ t$. Este hexámero cambia las posiciones de sus átomos uniéndose el N4 con el N6, quedando finalmente una geometría no lineal con el siguiente orden: AlNNNNAl, véase Figura A.1. El agregado que le sigue en estabilidad es el hexagonal alternado AlNAINAlN (H) con una energía de atomización de $3.16 \mathrm{eV} / a$ át, este resultado coincide con BelBruno ${ }^{33}$, quien también obtiene el hexámero alternado de estado electrónico singlete como más estable. Ambos agregados prefieren la menor multiplicidad para estabilizarse.

Tabla A.2. Multiplicidades de espín $\left(\mathrm{M}_{\mathrm{S}}\right)$ y energías de atomización $\left(\mathrm{E}_{\mathrm{at}}\right)$ en eV/át, de los agregados lineales.

\begin{tabular}{l|c|c} 
Agregado & $\mathbf{M}_{\mathbf{s}}$ & $\mathbf{E}_{\mathbf{a t}}$ \\
\hline AlN & 3 & 1.39 \\
\hline AlNN & 2 & 3.38 \\
\hline AlNAl & 2 & 2.60 \\
\hline NAlN & 4 & 1.61 \\
\hline AlAlN & 4 & 1.12 \\
\hline AlNNN & 1 & 3.73 \\
\hline NAlNN & 1 & 2.93 \\
\hline AlNNAl & 3 & 3.00 \\
\hline AlNNNAl & 2 & 3.17 \\
\hline AlNNAlN & 2 & 2.70
\end{tabular}

En la Tabla A.2 se muestran los resultados para los agregados de hasta cinco átomos cuya geometría inicialmente era lineal. Se observa que el dímero AlN se estabiliza en un estado electrónico triplete y el más estable de los trímeros es AlNN, de estado electrónico doblete, siguiéndole AlNAl, con estado electrónico es doblete y con una diferencia de $0.78 \mathrm{eV} / a ́ t$. De los tetrámeros el más estable es AlNNN, siguiéndole NAlNN, $0.8 \mathrm{eV} / a$ át por debajo, ambos con estado electrónico singlete. Este tetrámero, inicialmente NAINN, reacomoda sus átomos luego de la optimización, obteniéndose una estructura con el orden de átomos AlNNN (véase Figura A.2). 
Se observa una preferencia a estabilizar los agregados con mayor cantidad de átomos de nitrógeno en su estructura, y unidos entre sí. Con respecto a los pentámeros, el más estable es AlNNNAl, de estado electrónico doblete.

Tabla A.3. Multiplicidades de espín $\left(\mathrm{M}_{\mathrm{S}}\right)$ y energías de atomización $\left(\mathrm{E}_{\mathrm{at}}\right)$ en eV/át, de los agregados no lineales. $(\mathrm{R})$ significa rombo.

\begin{tabular}{l|c|c}
\multicolumn{1}{c|}{ Agregado } & $\mathbf{M}_{\mathbf{s}}$ & $\mathbf{E}_{\mathbf{a t}}$ \\
\hline NAlN & 4 & 2.30 \\
\hline AlNNN & 1 & 2.97 \\
\hline AlNAlN (R) & 1 & 3.00 \\
\hline NAlNN & 3 & 3.03 \\
\hline AlNNNA1 & 2 & 2.71 \\
\hline AlNNA1Al & 4 & 2.57 \\
\hline AlNNA1N & 2 & 2.63
\end{tabular}

Con respecto a los agregados no lineales optimizados (véase Figura A.3 para las estructuras finales), se obtuvo un solo trímero estable, NAlN, con estado electrónico triplete y energía de atomización de $2.30 \mathrm{eV} /$ át, muy por debajo del trímero lineal más estable. De los tetrámeros el más estable es NAINN, de estado electrónico triplete, encontrándose muy cercanos en energía el rombo alternado AlNAIN y AINNN, ambos de estado electrónico singlete. El agregado más estable no lineal de cinco átomos encontrado es el AlNNNAl, de estado electrónico doblete.

\section{B. Análisis poblacional de Mulliken}

A continuación se muestran los resultados del análisis poblacional de Mulliken, realizado con distintos conjuntos de funciones base, para todos los agregados obtenidos, con el fin de buscar comportamientos paralelos entre agregados estables y justificar la estabilidad encontrada. Asimismo, se busca una correlación entre las poblaciones y el crecimiento del agregado.

El análisis se realizó con distintos conjuntos de funciones base debido a que con el conjunto elegido originalmente $(6-311+\mathrm{g}(3 \mathrm{df}))$ las poblaciones de Mulliken resultaron la mayoría negativas (Tablas B.1 y B.2).

En los casos en los que se informa una sola multiplicidad es debido a que sólo esa llego a convergencia. 
Tabla B.1. Resultados del análisis poblacional de Mulliken, donde $\Sigma \mathrm{P}$ es la suma de las poblaciones de solapamiento del agregado lineal correspondiente. También se muestran las

\begin{tabular}{|c|c|c|c|c|c|c|}
\hline Agregado & $\mathbf{M}_{\mathbf{s}}$ & $\mathbf{E}_{\mathrm{at}}$ & $\sum_{6-311+g(3 d f)}^{\sum P}$ & $\begin{array}{c}\sum_{6-311+g(d)} \\
6\end{array}$ & $\underset{6-311 g(3 d f)}{\sum P}$ & $\sum_{6-311 g(d)}^{\sum P}$ \\
\hline \multirow{2}{*}{ AlN } & 1 & 0.82 & 0.902752 & 1.080854 & 1.351104 & 1.147368 \\
\hline & 3 & 1.39 & 0.223698 & 0.163041 & 0.462836 & 0.347716 \\
\hline \multirow[t]{2}{*}{ AlNN } & 2 & 3.38 & -3.332617 & -0.0450775 & 0.237797 & 0.487962 \\
\hline & 4 & 2.66 & -2.572673 & -0.438459 & 0.484729 & 0.471059 \\
\hline \multirow[t]{2}{*}{ AlNAl } & 2 & 2.60 & 0.471334 & 0.334652 & 0.99343 & 0.842796 \\
\hline & 4 & 1.78 & 0.366734 & -0.201782 & 0.730812 & 0.739096 \\
\hline \multirow[t]{2}{*}{ NAlN } & 2 & 0.05 & 0.51553 & 0.663634 & 1.014472 & 0.814172 \\
\hline & 4 & 1.61 & 0.405964 & 0.483812 & 0.939646 & 0.711436 \\
\hline AlAlN & 4 & 1.12 & 0.504788 & 0.416974 & 0.773144 & 0.481037 \\
\hline \multirow[t]{2}{*}{ AlNNN } & 1 & 3.73 & -3.938477 & -15.593827 & 0.738197 & 0.935044 \\
\hline & 3 & 2.96 & -3.008521 & -0.971507 & 0.796738 & 1.182081 \\
\hline NAINN & 1 & 2.93 & -3.878922 & -0.452325 & 0.617041 & 1.097505 \\
\hline \multirow[t]{2}{*}{ AlNNAl } & 1 & 2.83 & -9.904782 & -9.623854 & -0.590213 & 0.560337 \\
\hline & 3 & 3.00 & -9.539975 & -8.582497 & -0.35877 & 0.553517 \\
\hline \multirow[t]{2}{*}{ AlNNNAl } & 2 & 3.17 & -3.621632 & -3.0956621 & 0.108542 & 0.953616 \\
\hline & 4 & 2.70 & -1.559564 & -0.724453 & 0.55936 & 0.991114 \\
\hline \multirow[t]{2}{*}{ AlNNAIN } & 2 & 2.71 & -0.313978 & -23.897492 & 0.432689 & 1.068381 \\
\hline & 4 & 2.69 & -0.249343 & -21.586661 & 0.514157 & 1.042834 \\
\hline AlNNNAIN & 1 & 3.17 & -2.447663 & -18.714381 & 0.201868 & 1.318214 \\
\hline
\end{tabular}

Tabla B.2. Resultados del análisis poblacional de Mulliken, donde $\Sigma \mathrm{P}$ es la suma de las poblaciones de solapamiento del agregado no lineal correspondiente. También se muestran las multiplicidades de espín $\left(\mathrm{M}_{\mathrm{s}}\right)$ y energías de atomización $\left(\mathrm{E}_{\mathrm{at}}\right)$ en eV/át.

\begin{tabular}{|c|c|c|c|c|c|c|}
\hline Agregado & Ms & $\mid \begin{array}{c}\mathbf{E}_{\text {at }} \\
(\mathrm{eV} / \mathbf{a} t)\end{array}$ & $\sum_{6-311+g}^{\sum P}(3 d f)$ & $\sum_{6-311+g(d)}^{\sum P}$ & $\sum_{6-311 g(3 d f)}^{\sum P}$ & $\sum_{6-311 g(d)}^{\sum P}$ \\
\hline NAIN & 4 & 2.30 & -0.107206 & 0.447341 & 7.205392 & 0.528795 \\
\hline \multirow[t]{2}{*}{ AlNNN } & 1 & 2.97 & -1.346235 & 0.007426 & 0.483226 & 0.522888 \\
\hline & 3 & 2.96 & 0.807502 & -0.48128 & 0.449356 & 0.27652 \\
\hline AlNAIN (R) & 1 & 3.00 & -0.2993 & -0.137422 & -0.052727 & 0.325856 \\
\hline NAlNN & 3 & 3.03 & -0.820794 & 0.30061 & 1.114536 & 0.70525 \\
\hline AlNNNAl & 2 & 2.71 & -0.698741 & -1.630236 & 0.827724 & 0.52415 \\
\hline AlNNAlAl & 4 & 2.57 & -2.276928 & -1.387617 & 0.292203 & 0.549963 \\
\hline \multirow[t]{2}{*}{ AlNNAIN } & 2 & 2.63 & -1.439199 & -1.029474 & 0.677536 & 0.681465 \\
\hline & 4 & 2.61 & -0.534 & -0.719636 & 0.881597 & 0.692325 \\
\hline
\end{tabular}

No se ha encontrado una correlación entre el análisis poblacional de Mulliken y la estabilidad de los agregados, ni se ha encontrado por este análisis una justificación al patrón de crecimiento de los mismos. Aún más, se observa la tan conocida dependencia de este análisis con el conjunto de funciones base utilizado, sin poder siquiera conservar el signo del índice en un mismo agregado calculado con distintas funciones base. 


\section{COOP y DOS}

Los cálculos de COOP y densidad de estados se realizaron con programas realizados en el grupo de trabajo. A continuación se muestran gráficas de COOP y DOS correspondientes a cada uno de los casos lineales más estables (AlN, AlNN, ANNN, AlNNNAl y AlNNNAlN; ver Tablas A.1, A.2 y A.3). Las referencias se encuentran en el mismo gráfico, donde Efermi es la energía de Fermi correspondiente a cada agregado.
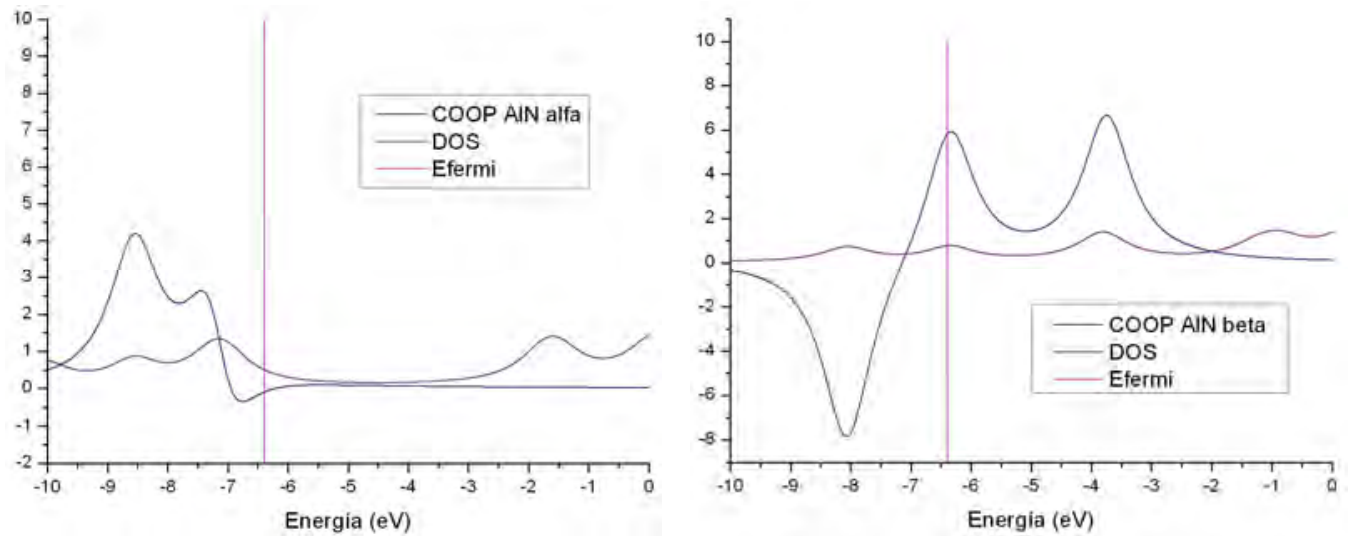

Figura C.1. Gráficos de COOP y DOS total en función de la energía del enlace Al-N, para el dímero AlN.
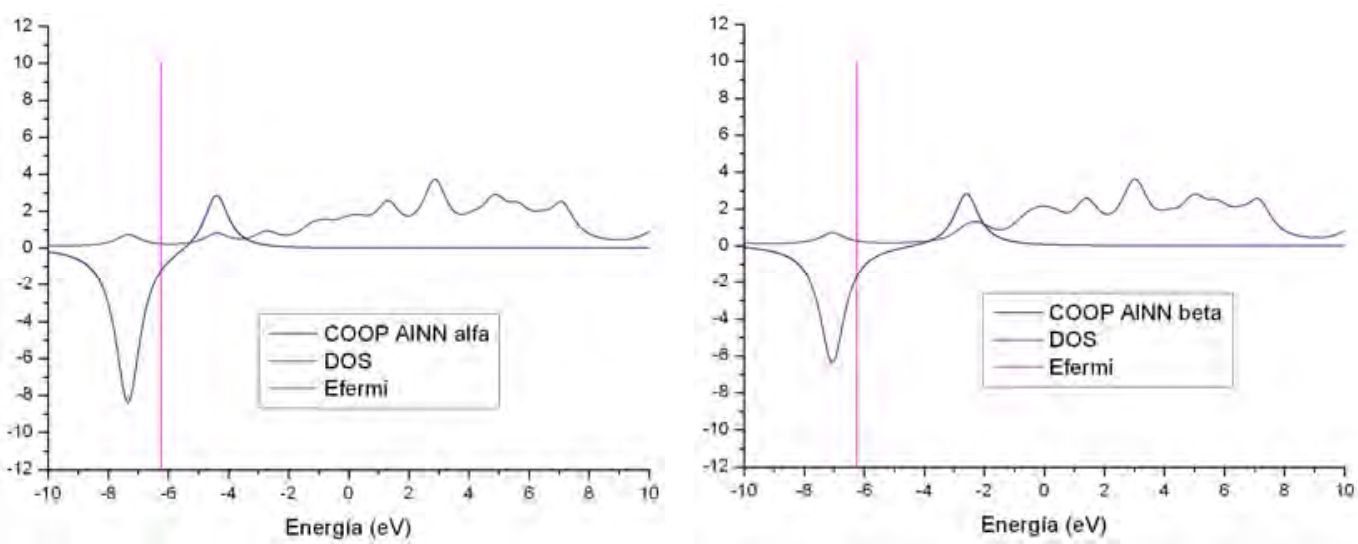

Figura C.2. Gráficos de COOP y DOS total en función de la energía del enlace Al-N para el trímero AlNN. 


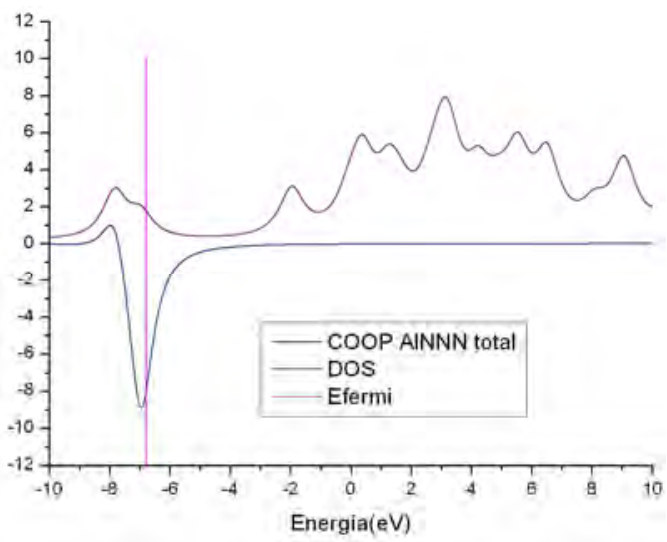

Figura C.3. Gráficos de COOP y DOS total en función de la energía del enlace Al-N para el tetrámero AlNNN.
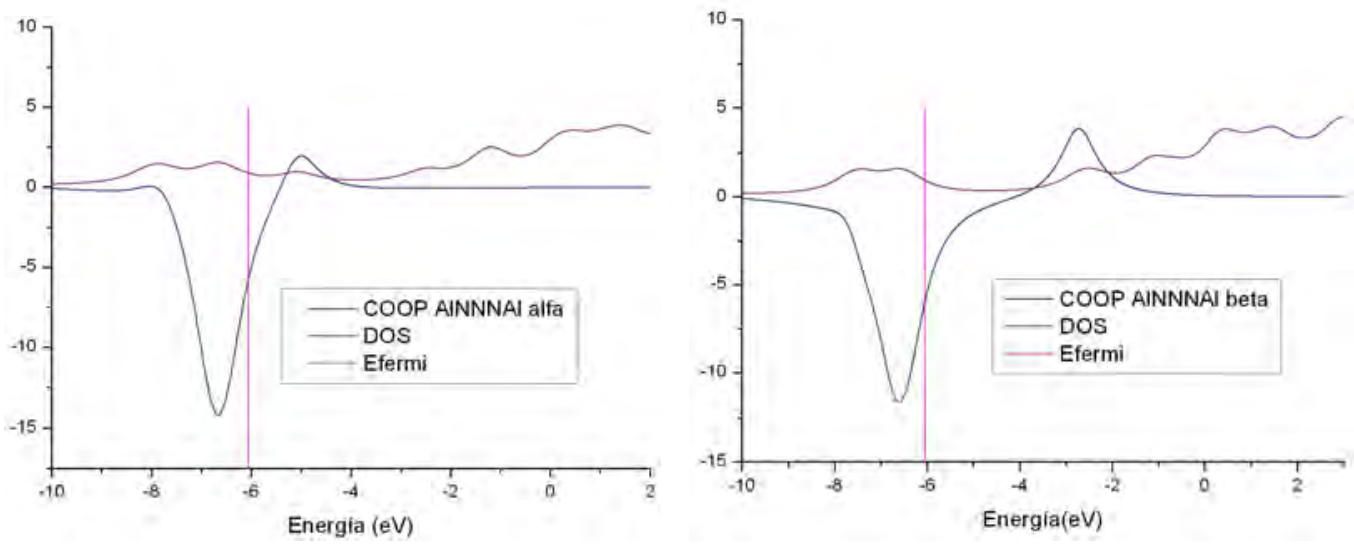

Figura C.4. Gráficos de COOP y DOS total en función de la energía del enlace Al-N para el pentámero AlNNNAl.

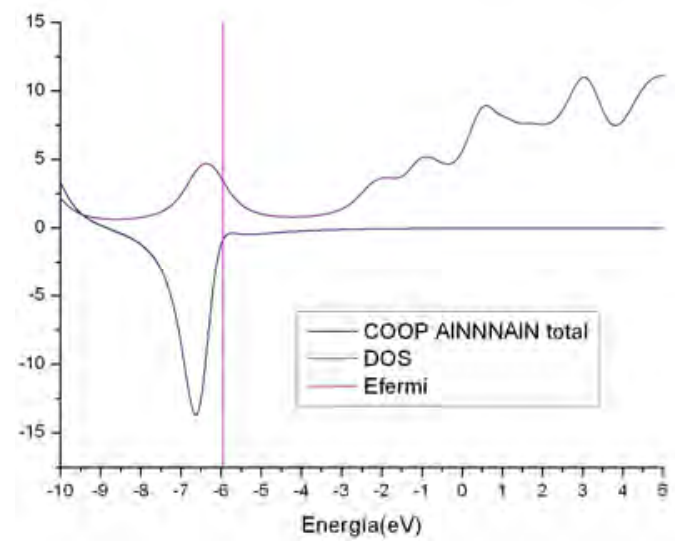

Figura C.5. Gráficos de COOP y DOS total en función de la energía del enlace AlN para el hexámero AINNNAIN. 

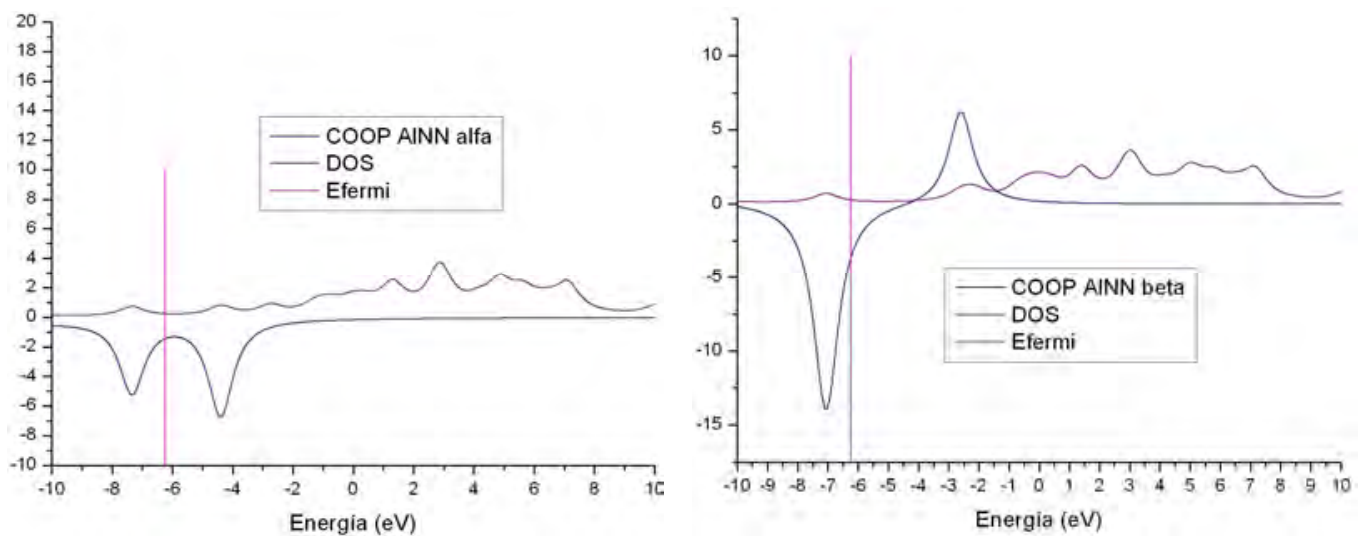

Figura C.6. Gráficos de COOP y DOS total en función de la energía del enlace N-N, para el trímero AlNN.

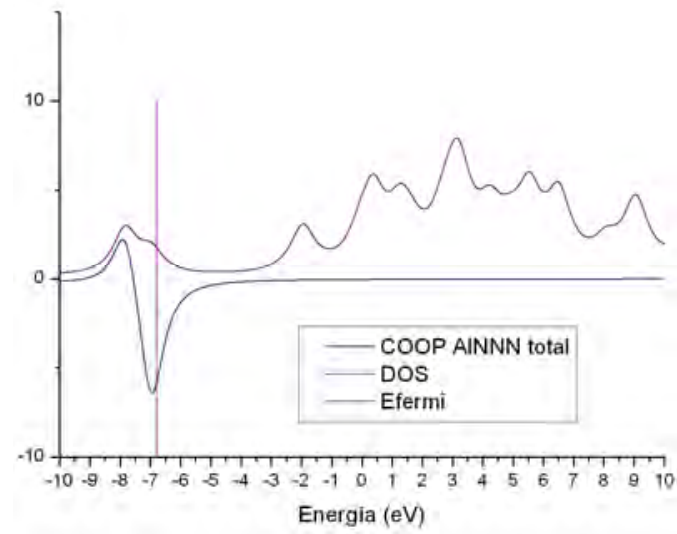

Figura C.7. Gráficos de COOP y DOS total en función de la energía del enlace N2-N3, para el tetrámero AlNNN.

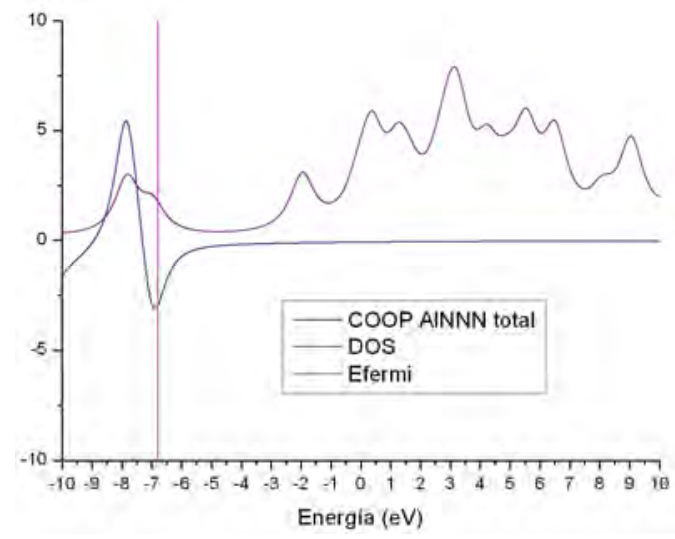

Figura C.8. Gráficos de COOP y DOS total en función de la energía del enlace N3-N4, para el tetrámero AlNNN. 

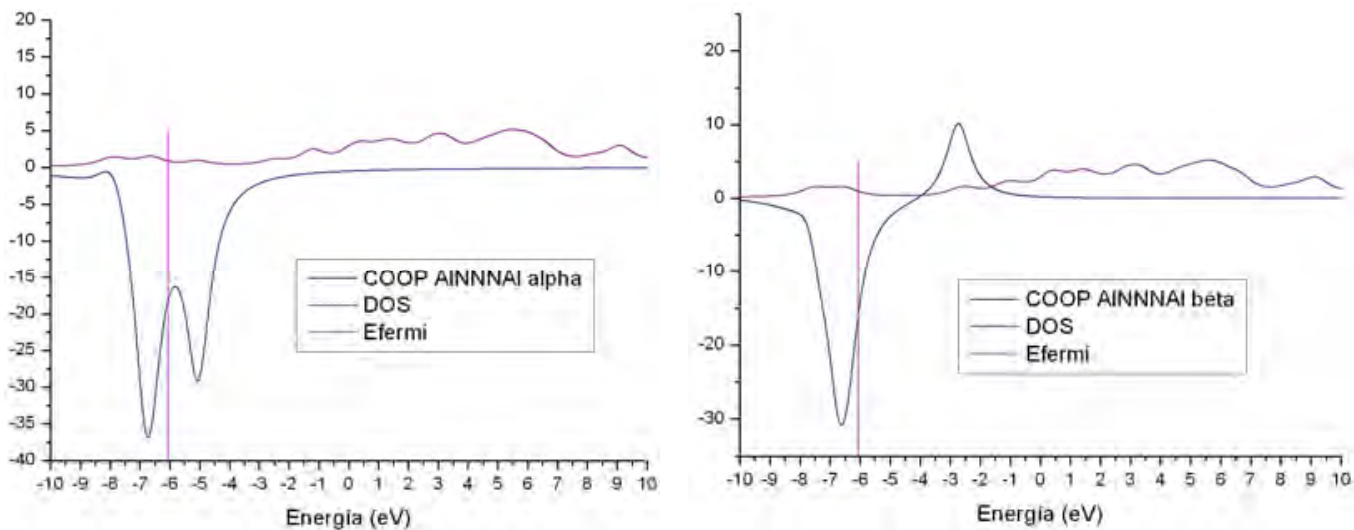

Figura C.9. Gráficos de COOP y DOS total en función de la energía del enlace N2-N3, para el pentámero AINNNAl.
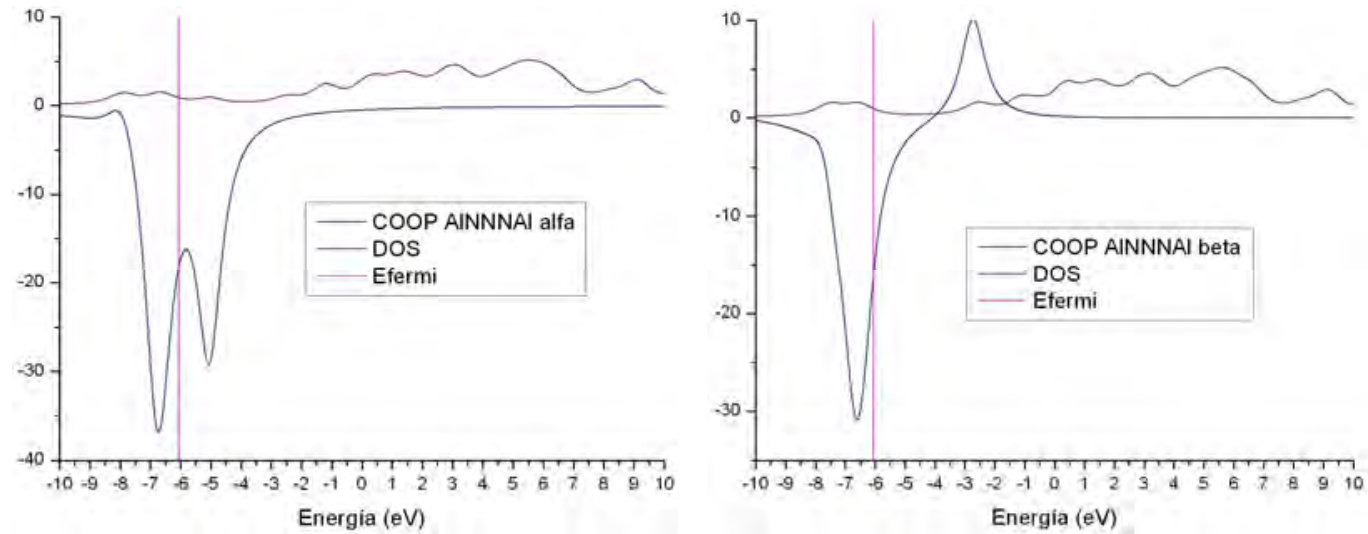

Figura C.10. Gráficos de COOP y DOS total en función de la energía del enlace N3-N4, para el pentámero AlNNNAl.
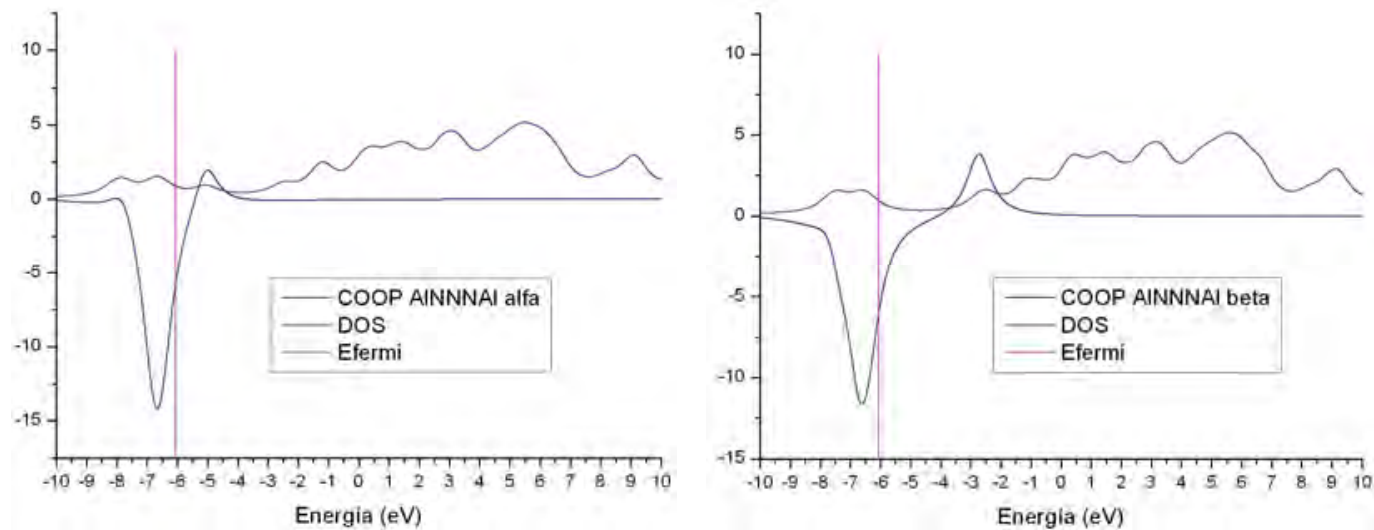

Figura C.11. Gráficos de COOP y DOS total en función de la energía del enlace N4-Al5, para el pentámero AlNNNAl. 


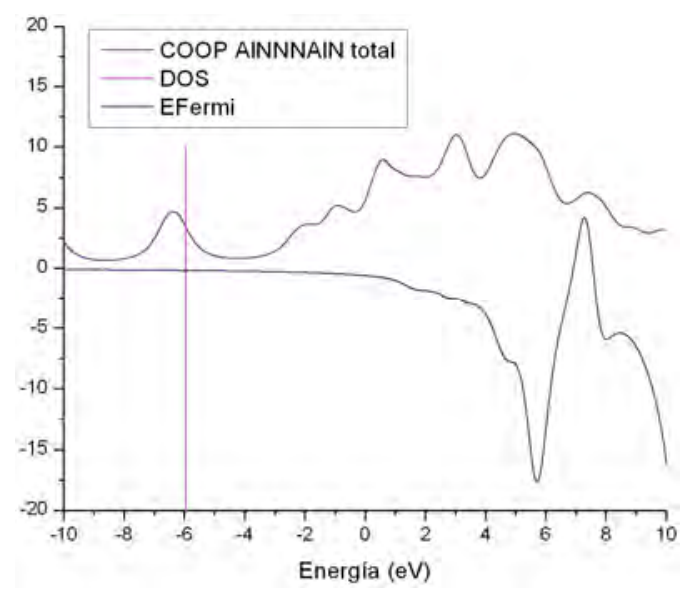

Figura C.12. Gráficos de COOP y DOS total en función de la energía del enlace N2-N3, para el hexámero AINNNAIN.

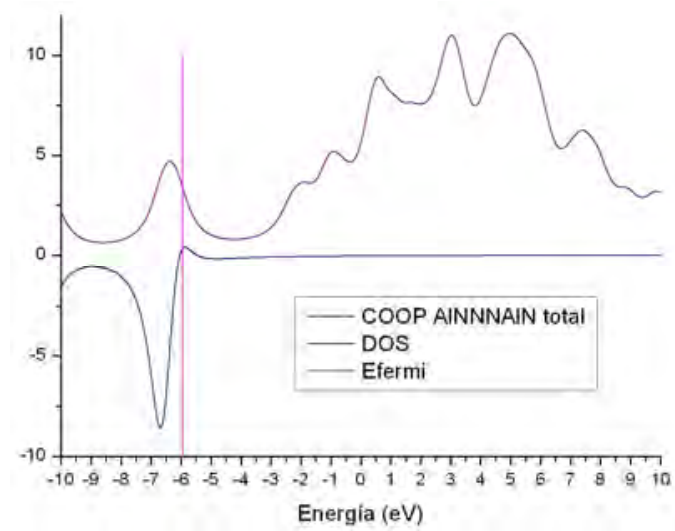

Figura C.13. Gráficos de COOP y DOS total en función de la energía del enlace N3-N4, para el hexámero AINNNAIN.

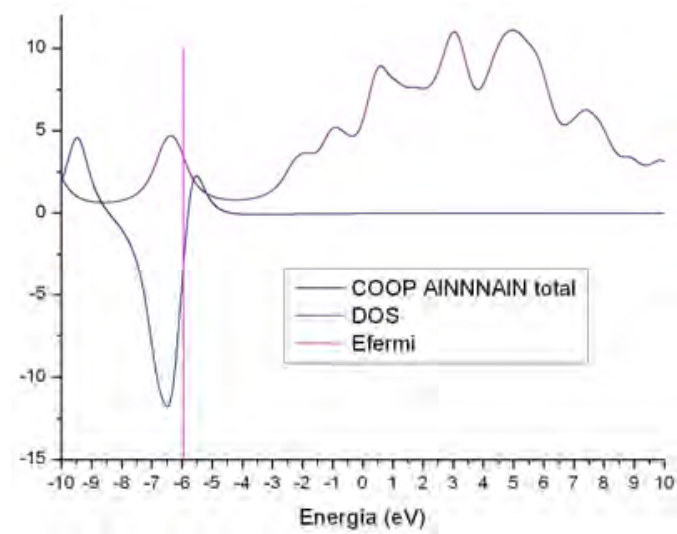

Figura C.14. Gráficos de COOP y DOS total en función de la energía del enlace N4-N6, para el hexámero AINNNAIN. 


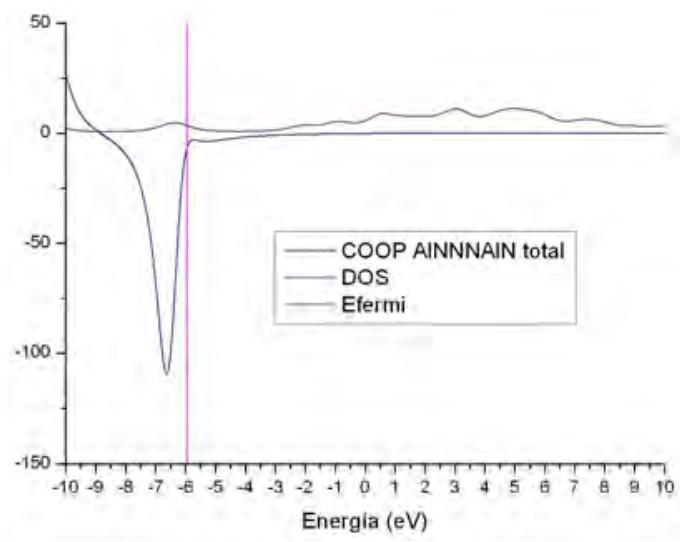

Figura C.15. Gráficos de COOP y DOS total en función de la energía del enlace N6-A15, para el hexámero AlNNNAIN.

Se observa en los gráficos obtenidos de COOP que las contribuciones de los orbitales generalmente son antienlazantes, hasta la energía de Fermi. No se encuentra justificación por este índice o por la densidad de estados a la estabilidad de los agregados. Tampoco se encuentra justificación al patrón de crecimiento de los agregados mediante el análisis de los gráficos de COOP y DOS.

\section{DFT conceptual}

\section{a) Afinidad electrónica, potencial de ionización, dureza y electronegatividad}

Se obtuvieron las afinidades electrónicas adiabáticas, potenciales de ionización adiabáticos, índices de dureza y electronegatividades. En cada caso se buscó la multiplicidad de espín que minimiza la energía de las especies iónicas. Se muestran los resultados obtenidos en las Tablas D.1 y D.2 para todos los agregados calculados.

Tabla D.1. Multiplicidades de los iones (Ms (-) y Ms (+)), afinidades electrónicas (AE), potenciales de ionización (PI), dureza $(\eta)$ y electronegatividades $(\chi)$ calculadas, todo en $\mathrm{eV}$, para los agregados lineales.

\begin{tabular}{l|c|c|c|c|c|c|c} 
Agregado & Ms & Ms (-) & AE & Ms (+) & PI & $\boldsymbol{\eta}$ & $\boldsymbol{\chi}$ \\
\hline AlN & 1 & 2 & -2.94 & 4 & 8.10 & 2.58 & 5.52 \\
& 3 & 2 & -1.74 & 4 & 8.71 & 3.49 & 5.23 \\
\hline AlNN & 2 & 3 & -0.83 & 1 & 6.80 & 2.98 & 3.82 \\
& 4 & 3 & -2.96 & 1 & 5.61 & 1.32 & 4.28 \\
\hline AlNAl & 2 & 1 & -2.33 & 1 & 8.02 & 2.84 & 5.18 \\
& 4 & 1 & -4.52 & 3 & 5.90 & 0.69 & 5.21 \\
\hline NAlN & 2 & 3 & -4.14 & 5 & 7.73 & 1.80 & 5.93 \\
& 4 & 3 & -2.46 & 5 & 8.96 & 3.25 & 5.71 \\
\hline AlAlN & 4 & 3 & -2.46 & 3 & 6.65 & 2.10 & 4.56 \\
\hline AlNNN & 1 & 2 & -0.27 & 2 & 9.38 & 4.55 & 4.83 \\
& 3 & 2 & -3.25 & 2 & 6.08 & 1.41 & 4.67 \\
\hline NAlNN & 1 & 2 & -2.52 & 4 & 7.73 & 2.61 & 5.12 \\
\hline AlNNAl & 1 & 2 & -1.77 & 2 & 6.52 & 2.37 & 4.15 \\
& 3 & 2 & -1.16 & 2 & 7.41 & 3.13 & 4.29 \\
\hline
\end{tabular}




\begin{tabular}{l|l|l|l|l|l|l|l}
\hline AlNNNA1 & 2 & 1 & -1.19 & 1 & 7.10 & 2.96 & 4.14 \\
& 4 & 1 & -3.73 & 3 & 5.69 & 0.98 & 4.71 \\
\hline AlNNA1N & 2 & 3 & -2.08 & 3 & 8.03 & 2.97 & 5.05 \\
& 4 & 3 & -2.15 & 3 & 8.01 & 2.93 & 5.08 \\
\hline AlNNNAIN & 1 & 2 & -0.82 & 2 & 7.92 & 3.55 & 4.37
\end{tabular}

En los agregados de geometría inicial lineal, en todos los casos en que se obtuvieron agregados con dos multiplicidades diferentes, el de mayor dureza es el que resulta más estable. No obstante, al comparar cada tamaño de agregado por separado, se observa que en los trímeros, el de mayor dureza NAIN de estado electrónico cuatriplete, siendo que éste no es el más estable encontrado (ver Tabla A.1). En los tetrámeros se cumple que el agregado más estable, AlNNN de estado electrónico singlete, posee mayor dureza; y no se cumple esto en los pentámeros.

Tabla D.2. Multiplicidades de los iones de más baja energía (Ms (-) y Ms (+)), afinidades electrónicas (AE), potenciales de ionización (PI), ambos en eV, dureza $(\eta) \mathrm{y}$ electronegatividades $(\chi)$ calculadas, todo en $\mathrm{eV}$, para los agregados no lineales.

\begin{tabular}{l|c|c|c|c|c|c|c} 
Agregado & Ms & Ms (-) & AE & Ms (+) & PI & $\boldsymbol{\eta}$ & $\boldsymbol{\chi}$ \\
\hline NAlN & 4 & 1 & -3.25 & 3 & 7.01 & 1.88 & 5.13 \\
\hline AlNNN & 1 & 2 & -0.16 & 2 & 9.27 & 4.55 & 4.71 \\
& 3 & 2 & -1.14 & 2 & 8.96 & 3.91 & 5.05 \\
\hline AlNAlN (R) & 1 & 2 & -0.49 & 2 & 8.15 & 3.83 & 4.32 \\
\hline NAlNN & 3 & 2 & -2.41 & 2 & 8.12 & 2.85 & 1.4 \\
\hline AlNNNAl & 2 & 1 & -0.83 & 1 & 7.80 & 3.48 & 4.32 \\
\hline AlNNA1Al & 4 & 3 & -1.41 & 3 & 6.13 & 2.36 & 3.77 \\
\hline AlNNA1N & 2 & 3 & -2.19 & 3 & 7.61 & 2.71 & 4.90 \\
& 4 & 3 & -2.44 & 3 & 7.59 & 2.58 & 5.01
\end{tabular}

Con respecto a los agregados de geometría inicial no lineal, en los dos casos en que se obtuvieron agregados con dos multiplicidades diferentes, el de mayor dureza es el que resulta más estable. Comparando todos los tetrámeros entre sí, se observa que el agregado más estable no es el que tiene mayor dureza; aunque sí se cumple esto si observamos los pentámeros.

\section{b) Funciones de Fukui}

Las funciones de Fukui fueron calculadas con un programa desarrollado en el grupo de trabajo. Se muestran los resultados para los mismos casos de la sección C.

Tabla D.3. Funciones de Fukui, en |e|, de los agregados más estables de cada caso lineal.

\begin{tabular}{c|c|c|c} 
AlN & $f(+)$ & $f(-)$ & $f(0)$ \\
\hline $\mathrm{A} 1$ & 0.474159 & 0.210225 & 0.342192 \\
\hline $\mathrm{N}$ & 0.525847 & 0.789783 & 0.657815
\end{tabular}

\begin{tabular}{c|c|c|c} 
AlNN & $f(+)$ & $f(-)$ & $f(0)$ \\
\hline $\mathrm{A} 1$ & 0.596155 & 0.212991 & 0.404573 \\
\hline $\mathrm{N}$ & 0.190863 & 0.261318 & 0.226091 \\
\hline $\mathrm{N}$ & 0.212991 & 0.281870 & 0.247431
\end{tabular}




\begin{tabular}{c|c|c|c} 
AlNNN & $f(+)$ & $f(-)$ & $f(0)$ \\
\hline $\mathrm{Al}$ & 0.822598 & 0.131665 & 0.477132 \\
\hline $\mathrm{N}$ & 0.006038 & -0.047547 & -0.020755 \\
\hline $\mathrm{N}$ & 0.039697 & 0.057819 & 0.048758 \\
\hline $\mathrm{N}$ & 0.131665 & 0.004465 & 0.068065
\end{tabular}

\begin{tabular}{c|c|c|c} 
AlNNNAl & $f(+)$ & $f(-)$ & $f(0)$ \\
\hline $\mathrm{A} 1$ & 0.241966 & 0.165238 & 0.203602 \\
\hline $\mathrm{N}$ & 0.165238 & 0.241966 & 0.203602 \\
\hline $\mathrm{N}$ & 0.185599 & 0.145986 & 0.165793 \\
\hline $\mathrm{N}$ & 0.165238 & 0.309169 & 0.237203 \\
\hline $\mathrm{A} 1$ & 0.241966 & 0.117842 & 0.179904
\end{tabular}

\begin{tabular}{c|c|c|c} 
AlNNNAlN & $f(+)$ & $f(-)$ & $f(0)$ \\
\hline $\mathrm{Al}$ & 0.280608 & 0.280579 & 0.280593 \\
\hline $\mathrm{N}$ & 0.108214 & 0.108225 & 0.108219 \\
\hline $\mathrm{N}$ & 0.111196 & 0.086920 & 0.099058 \\
\hline $\mathrm{N}$ & 0.111178 & 0.086910 & 0.099044 \\
\hline $\mathrm{A} 1$ & 0.280579 & 0.071160 & 0.175869 \\
\hline $\mathrm{N}$ & 0.108225 & 0.341907 & 0.225066
\end{tabular}

Se obtuvieron las funciones de Fukui de los agregados, función de Fukui nucleofílica $f(+)$, electrofílica $f(-)$ y la función que mide reactividad hacia un radical $f(0)$. En el dímero AlN la reactividad hacia un nucleófilo está concentrada en el átomo de nitrógeno, como así también la reactividad hacia un electrófilo y un radical. En el trímero AlNN, el átomo de aluminio concentra la reactividad hacia un nucleófilo y hacia un radical, mientras que los átomos de nitrógeno poseen reactividad más marcada hacia un electrófilo. En el tetrámero AlNNN el aluminio posee los valores mayores de los tres tipos de funciones de Fukui, comportándose como se comporta en el dímero. En el pentámero AlNNNAl los átomos de aluminio poseen una reactividad marcada hacia nucléfilos y el N4 concentra los valores mayores de funciones de Fukui electrofílica y radical. Con respecto al hexámero estudiado, los átomos de aluminio se comportan como en el pentámero, concentrando la reactividad hacia nucleófilos y el Al1 posee reactividad marcada hacia un radical, mientras que el N6 posee la mayor reactividad hacia un electrófilo. A pesar de haber descripto los sistemas mediante estos índices de reactividad, no se encuentra el camino de crecimiento de los agregados mediante la utilización de éstas funciones. Además, se han encontrado índices negativos en la función de Fukui electrofílica (caso AlNNN), lo cual no tiene sentido físico.

\section{Conclusiones del análisis preliminar de agregados de $\mathrm{Al} / \mathrm{N}$}

Se ha encontrado en los sistemas inicialmente lineales que los sistemas con mayor cantidad de átomos de nitrógeno unidos entre sí son más estables. No se observa este comportamiento en agregados inicialmente no lineales.

El análisis poblacional de Mulliken no muestra un comportamiento paralelo a la estabilidad de los clusters. La dependencia de los resultados de éste análisis poblacional con el conjunto base utilizado es muy marcado, no pudiéndose conservar el signo del resultado, siquiera. 
Los análisis de COOP y DOS tampoco contribuyen significativamente a la explicación de la estabilidad de los clusters obtenidos. COOP muestra contribuciones antienlazantes en todos los casos, lo que se deba posiblemente a que este análisis se calcula basado en poblaciones de Mulliken. Por otro lado, DOS no muestra tampoco tendencias que permitan explicar la estabilidad de los agregados y encontrar el camino de crecimiento de los mismos.

Con respecto a los índices de DFT conceptual, los resultados muestran que el principio de máxima dureza se cumple sólo para los agregados inicialmente lineales de cuatro átomos y para los pentámeros no lineales. Se encuentra un comportamiento paralelo a la estabilidad de un agregado respecto del mismo agregado con otra multiplicidad. A pesar de no encontrarse una explicación a la estabilidad, se obtuvieron datos de potenciales de ionización, afinidades electrónicas y electronegatividades, que pueden resultar útiles con posterioridad. Las funciones de Fukui muestran que existe una preferencia en estos sistemas a que los átomos de aluminio sean reactivos hacia nucleófilos y los átomos de nitrógeno sean reactivos hacia electrófilos. Se han encontrado funciones de Fukui electrofílicas con signo negativo, lo cual no tiene sentido físico. 


\section{APÉNDICE 3: Análisis preliminares de agregados de B/N: el uso de distintos métodos de análisis de la función de onda}

Se estudiaron las geometrías lineales, hexagonales y octaédricas de $\mathrm{B}_{3} \mathrm{~N}_{3}$, en todas las posibles combinaciones de $\mathrm{B}$ y $\mathrm{N}$, de multiplicidad de espín electrónica 1 y 3 . Los agregados han sido estudiados neutros. Se realizaron análisis de AIM, ELF y Análisis Poblacional de Mulliken ${ }^{121}$.

Las optimizaciones de geometría y cálculos de frecuencia de los agregados, tanto neutros como iónicos, se realizaron utilizando DFT, mediante el funcional B3LYP. Los conjuntos de funciones base utilizados fueron $6-311+\mathrm{G}(3 \mathrm{df})$. Los cálculos se llevaron a cabo utilizando el paquete de programas Gaussian 03.

Los análisis de AIM y ELF, fueron llevados a cabo con los paquetes de programas AIMPAC $^{122}$ y $\mathrm{ToPMoD}^{123}$, respectivamente. El análisis de Mulliken se llevó a cabo implementando el programa MullPop ${ }^{124}$.

\section{A. Optimización de Geometrías}

Se obtuvieron las geometrías de menor energía, multiplicidades de espín y energías de atomización. De los cálculos realizados informamos la distribución de $\mathrm{B}_{3} \mathrm{~N}_{3}$ más estable para las geometrías lineal y hexagonal, BNBNBN, Tablas A.1 y A.2, cuyas distancias de enlace y diferencias energéticas se muestran en la Figura A.1. Se encontró que la geometría octaédrica se deforma a una geometría hexagonal, por lo que no se han continuado los estudios de la misma.

Tabla A.1. Energías de atomización (en eV/át) obtenidas de las distintas combinaciones de $\mathrm{B}$ y $\mathrm{N}$ optimizadas, para los hexágonos, donde $\mathrm{M}_{\mathrm{S}}$ es la multiplicidad..

\begin{tabular}{l|c|c} 
& $\mathbf{M}_{\mathbf{S}}$ & $\mathbf{E}_{\mathbf{a t}}$ \\
\hline BBBNNN & 3 & 3.89 \\
\hline & 1 & 3.90 \\
\hline BNBBNN & 3 & 4.29 \\
\hline & 1 & 4.47 \\
\hline BNBNBN & 3 & 4.68 \\
\hline & 1 & 5.20
\end{tabular}

Tabla A.2. Energías de atomización (en eV/át) obtenidas de las distintas combinaciones de $\mathrm{B}$ y $\mathrm{N}$ optimizadas, para los hexámeros lineales, donde $\mathrm{M}_{\mathrm{S}}$ es la multiplicidad.

\begin{tabular}{c|c|c} 
& $\mathbf{M}_{\mathbf{S}}$ & $\mathbf{E}_{\mathbf{a t}}$ \\
\hline BBBNNN & 1 & 3.88 \\
\hline & 3 & 3.82 \\
\hline BBNBNN & 1 & 4.10 \\
\hline & 3 & 4.20
\end{tabular}




\begin{tabular}{l|l|l} 
BBNNBN & 1 & 3.70 \\
\hline & 3 & 3.83 \\
\hline BBNNNB & 1 & 3.51 \\
\hline & 3 & 3.51 \\
\hline BNBBNN & 1 & 4.35 \\
\hline & 3 & 4.50 \\
\hline BNBNBN & 1 & 4.47 \\
\hline & 3 & 4.70 \\
\hline BNBNNB & 1 & 4.07 \\
\hline & 3 & 4.30 \\
\hline BNNBBN & 1 & 3.88 \\
\hline & 3 & 4.11 \\
\hline NBBBNN & 1 & 4.07 \\
\hline & 3 & 4.20 \\
\hline NBBNBN & 1 & 4.22 \\
\hline & 3 & 4.43
\end{tabular}

De las Tablas A.1 y A.2 se puede observar que el estado fundamental de ambas geometrías es el que presenta alternancia en el orden atómico, es decir BNBNBN. El hexámero lineal BBNNNB de estado electrónico singlete presenta una diferencia de sólo $0.007 \mathrm{eV} /$ át con respecto al estado electrónico triplete.
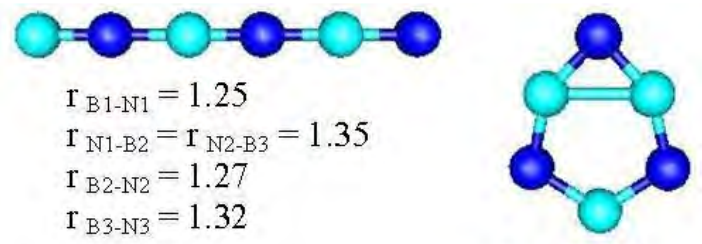

$$
\mathrm{r}_{\mathrm{B}-\mathrm{N}}=1.35
$$

Figura A.1. Geometrías de equilibrio de los agregados más estables; donde las distancias de enlace están expresadas en $\AA$. Los átomos de boro se muestran en celeste y los de nitrógeno en azul. Los átomos se numeran de izquierda a derecha.

Para explicar la estabilidad de la geometría hexagonal con respecto a la lineal, se recurrió al análisis de la función de onda de las distintas combinaciones de $\mathrm{B}$ y $\mathrm{N}$ de geometría hexagonal mediante diversos métodos.

\section{B. Análisis poblacional de Mulliken}

El análisis poblacional de Mulliken es uno de los métodos más ampliamente usados de análisis de la función de onda ${ }^{125}$. En la Tabla B.1 se muestran los resultados obtenidos para los hexágonos. 
Tabla B.1. Resultados del análisis poblacional de Mulliken, donde $\mathrm{M}_{\mathrm{S}}$ es la multiplicidad, $\mathrm{Q}$ la carga promedio de nitrógeno y de boro en $|\mathrm{e}|$, y $\Sigma \mathrm{P}$ es la suma de las poblaciones de solapamiento del hexágono neutro correspondiente. También se muestra la energía de atomización, en eV.

\begin{tabular}{c|c|c|c|c|c} 
& $\mathbf{M}_{\mathbf{S}}$ & $\mathbf{E}_{\text {at }}$ & $\mathbf{Q} \mathbf{N}$ & $\mathbf{Q} \mathbf{B}$ & $\mathbf{\Sigma} \mathbf{P}$ \\
\hline BBBNNN & 3 & 3.89 & $-0,269422$ & 0,269422 & $-0,698218$ \\
\hline & 1 & 3.90 & $-0,237507$ & 0,237507 & $-0,310941$ \\
\hline BNBBNN & 3 & 4.29 & $-0,332591$ & 0,332592 & 0,106804 \\
\hline & 1 & 4.47 & $-0,367208$ & 0,367208 & 1,364442 \\
\hline BNBNBN & 3 & 4.68 & $-0,447341$ & 0,447342 & 1,70409 \\
\hline & 1 & 5.20 & $-0,543099$ & 0,543099 & 2,002666
\end{tabular}

Analizando los datos de la Tabla B.1 se observa que la suma de las poblaciones de solapamiento muestra un comportamiento paralelo al de la estabilidad relativa de los agregados.

\section{C. Átomos en Moléculas}

En la Tabla C.1 se presentan los resultados de la suma de las densidades de los puntos críticos $\rho_{T}(r)$, calculadas con AIM, de los agregados estudiados con sus correspondientes energías de atomización y multiplicidades de espín. No se encontró un comportamiento de $\rho_{T}(r)$ que se corresponda con el de la energía.

Tabla C.1. Suma de densidades de puntos críticos, en u.a $\left(\rho_{T}(r)\right)$. También se muestran las energías de atomización $\mathrm{E}_{\mathrm{at}}$ (en eV/át) y multiplicidades de espín $\left(\mathrm{M}_{\mathrm{S}}\right)$.

\begin{tabular}{c|c|c|c} 
& $\mathbf{M}_{\mathbf{S}}$ & $\mathbf{E}_{\mathbf{a t}}$ & $\rho_{T}(r)$ \\
\hline BBBNNN & 3 & 3.89 & 1,166205 \\
\hline & 1 & 3.90 & 1,278890 \\
\hline BNBBNN & 3 & 4.29 & 0,791800 \\
\hline & 1 & 4.47 & 0,873027 \\
\hline BNBNBN & 3 & 4.68 & 0,881070 \\
\hline & 1 & 5.20 & 0,894160
\end{tabular}

En la Figura C.1 se muestran los valores de laplaciano de la densidad, elipticidad y densidad correspondiente a cada punto crítico, calculados para el hexágono BNBNBN en estados electrónicos triplete y singlete, respectivamente. Se observa que estos valores no justifican la estabilidad de los agregados. 

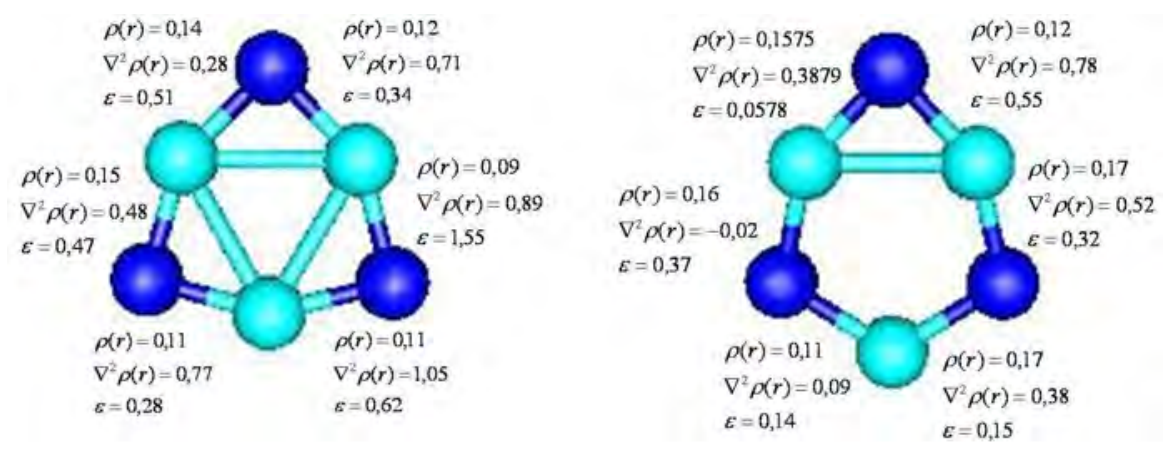

Figura C.1. $\rho(r), \nabla^{2} \rho(r)$ y $\varepsilon$ para el hexágono BNBNBN con estados electrónicos singlete y triplete, respectivamente, en u.a.

\section{Función de localización electrónica}

En el esfuerzo por entender la estabilidad del hexágono BNBNBN de estado electrónico singlete, se realizó el análisis topológico de ELF. En el caso de las geometrías menos estables se encontró que existen pares libres sobre los átomos de boro y que la población electrónica se encuentra distribuida en basins disinápticos y polisinápticos. En el caso del hexágono más estable, BNBNBN singlete, se observa que la población electrónica se encuentra mejor distribuida en basins disinápticos y que los pares libres se encuentran sólo sobre los átomos de nitrógeno. Esto supone que la estabilidad de este sistema se debe a una mejor localización electrónica. En la Figura D.1 se pueden ver los pares libres de los átomos de nitrógeno como era esperado.

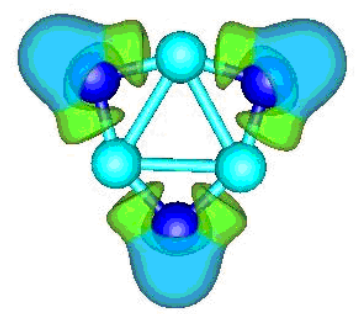

Figura D.1. Gráfico de ELF de la geometría más estable de $\mathrm{B}_{3} \mathrm{~N}_{3}$ en u.a.

Por lo expresado anteriormente se evidencia que ELF es un buen indicativo de la distribución electrónica sobre el agregado más estable, encontrándose los pares libres sobre átomos de nitrógeno, como es esperable. Aún así, ELF no aporta datos significativos o novedosos sobre los agregados restantes y sobre este mismo, más allá de la obviedad de obtener pares libres sobre los nitrógenos.

\section{Conclusiones del análisis preliminar para agregados de $\mathbf{B} / \mathbf{N}$}

Se optimizaron las distintas distribuciones de $\mathrm{B}_{3} \mathrm{~N}_{3}$ lineales y hexagonales, obteniéndose las geometrías de equilibrio. La conformación más estable encontrada es BNBNBN de estado electrónico singlete, siguiendo en estabilidad el agregado de estado electrónico triplete.

La suma de las poblaciones de solapamiento obtenidas según el análisis poblacional de Mulliken tiene un comportamiento paralelo a la energía del sistema, explicando que cuanto 
más fuertes son las uniones interatómicas, más estable es el sistema, como sucede con el hexágono BNBNBN en su estado fundamental.

Los análisis de las funciones de onda realizados con AIM y con ELF no muestran una correlación directa entre los datos y la estabilidad del BNBNBN de estado electrónico singlete.

En el caso de AIM, no se encontró correlación directa entre los valores de suma densidades de puntos críticos calculadas o valores de elipticidad, densidad de cada punto crítico y laplaciano de la misma con la estabilidad de los agregados.

En el caso de ELF, se encontró que en el hexágono más estable los pares libres se encuentran sobre átomos de nitrógeno y que la población electrónica se encuentra distribuída en basins disinápticos. 
${ }^{1}$ Y. Xie, Y. T. Qian, W. Z. Wang, S. Y. Zhang y Y. H. Zhang, Science 272 (1996) 1926.

${ }^{2}$ F. Xu, Y. Xie, X. Zhang, S. Y. Zhang y L. Shi, New J. Chem. 27 (2003) 565.

${ }^{3}$ T. Matsuoka, Adv. Mater. 8 (1996) 469.

${ }^{4}$ A. Yamamoto, M. Tsujino, M. Okhubo y A. Hashimoto, Sol. Energy Mater. 35 (1994) 53.

${ }^{5}$ a - A. C. Jones, C. R. Whitehouse y J. S. Roberts, Chem. Vapor Dep. 1 (1995) 65; b - I. Akasaki y H. Amano, J. Cryst. Growth 146 (1995) 455.

${ }^{6}$ J. E. M. Dougall, H. Eckert, G. D. Stucky, N. Herron, Y. Wang, K. Moller y T. Bein, J. Am. Chem. Soc. 111 (1989) 8006.

${ }^{7}$ S. R. Aubuchon, A. T. McPhail, R. L. Wells, J. A. Giambra y J. R. Bowser, Chem. Mater. 6 (1) (1994) 82.

${ }^{8}$ S. S. Kher y R. L. Wells, Material Research Society Symposium Proceedings 351 (1994) 293.

${ }^{9}$ R. E. Treece, G. S. Macala, L- Rao, D. Franke, H. Eckert y R. B. Kaner, Inorg. Chem. 32 (12) (1993) 2745.

${ }^{10}$ R. E. Treece, G. S. Macala y R. B. Kaner, Chem. Mater. 4 (1) (1992) 9.

${ }^{11}$ S. M. Gao, Y. xie, J. Lu, G. A. Du, W. He, D. L. Cui, B. B. Huang y M. H. Jiang, Inorg. Chem. 41 (7) (2002) 1850.

${ }^{12}$ H. Bonnemann, W. Brijoux y T. Joussen, Ang. Chem-Int. Ed. In. En. 29 (3) (1990) 273.

${ }^{13}$ P. Yan, Y. Xie, W. Z. Wang, F. Y. Liu y Y. T. Qian, J. Mater. Chem. 9 (8) (1999) 1831.

${ }^{14}$ M. Green, Curr. Opin. Solid State Mater. Sci., 6 (4) (2002) 355.

${ }^{15}$ Y. T. Qian, Adv. Mater. 11 (13) (1999) 1101.

${ }^{16}$ S. M. Gao, J. Lu, N. Chen, Y. Zhao y Y. Xie, Chem. Commun. 24 (2002) 3064.

${ }^{17}$ Y. J. Xiong, Y. Xie, Z. Q. Li, X. O. Li y R. Zhang, New J. Chem. 28 (2) (2004) 214.

${ }^{18}$ Y. N. Xia, P. D. Yang, Y. G. Sun, Y. Y. Wu, B. Mayers, B. Gates, Y. D. Yin, F. Kim y Y. Q. Yan, Adv. Mater. 15 (5) (2003) 353.

${ }^{19}$ C. Z. Wu, Q. Yang, C. Huang, D. Wang, P. Yin, T. W. Li y Y. Xie, Jo. Solid State Chem. 177 (10) (2004) 3522.

${ }^{20}$ R. Z. Ma, Y. Bando y T. Sato, Adv. Mater. 14 (5) (2002) 366.

${ }^{21}$ L. Q. Xu, Y. Y. Peng, Z. Y. Meng, W. C. Yu, Z. Y. Zhang, X. M. Liu y Y. T. Qian, Chem. Mater. 15 (13) (2003) 2675.

${ }^{22}$ X. J. Wang, Y. Xie y Q. X. Guo, Chem. Commun. 21 (2003) 2689.

${ }^{23}$ F. Xu, Y. Xie, X. Zhang, S. Y. Zhang, X. M. Liu, W. Xi y X. B. Tian, Adv. Funct. Mater. 14 (5) (2004) 464.

${ }^{24}$ Y. J. Xiong, U. Xie, Z. Q. Li, X. X. Li y S. M. Gao, Chemistry-A European Journal 10 (3) (2004) 654.

${ }^{25}$ Y. D. Li, X. L. Li, Z. X. Deng, B. C. Zhou, S. S. Fan, J. W. Wang y X. M. Sun, Ang. Chem-Int. Ed. In. En. 41 (2) (2002) 333.

${ }^{26}$ S. M. Gao, J. Lu, Y. Zhao, N. Chen y Y. Xie, Chem. Commun. 23 (2002) 2880.

${ }^{27}$ S. M. Gao, J. Lu, Y. Zhao, N. Chen y Y. Xie, Eur. J. Inorg. Chem. 9 (2003) 1822.

${ }^{28}$ S. M. Gao, Y. Xie, L. Y. Zhu y X. B. Tian, Inorg. Chem. 42 (17) (2003), 5442.

${ }^{29}$ S. M. Gao, Y. Xie, L. Y. Zhu y X. B. Tian, Eur. J. Inorg. Chem. 3 (2004), 557.

${ }^{30}$ P. Hohenberg y W. Kohn, Phys. Rev. 136 (1964), B864.

${ }^{31}$ R. G. Parr y W. Yang, Density Functional Theory of atoms and molecules, Oxford University Press and Clarendon Press (1989); L. J. Bartolotti y K. Flurchick, Rev. Comput. Chem. 7 (1996), 187; A. St-Amant, Rev. Comput. Chem. 7 (1996), 217; T. Ziegler, Chem. Rev. 91 (1991), 651; E. J. Baerendes y O. V. Gritsenko, J. Phys. Chem. 101 (1997), 5383.

${ }^{32}$ C. J. Cramer, Essentials of Computational Chemistry: Theories and Models $2^{\text {nd }}$ Edition, John Wiley \& Sons Ltd. (2004).

${ }^{33}$ L. H. Thomas, Proc. Camb. Phil. Soc. 23 (1927) 542.

${ }^{34}$ E. Fermi, Rend. Accad. Lincei 6 (1927), 602.

35 J. C. Slater, Phys. Rev. 81 (1951), 385.

${ }^{36}$ A. Szabo y N. S. Ostlund, Modern Quantum Chemistry: Introduction to Advanced Electronic Structure

Theory, Revised Edition, McGraw-Hill (1989).

${ }^{37}$ W. Kohn y L. J. Sham, Phys. Rev. 140 (1965) A1133.

${ }^{38}$ M. Levy y J. P. Perdew, en Density Functional Methods in Physics, ed. R. M. Dreizler y J. da Providencia, Plenum (1985).

${ }^{39}$ Q. Zhao, R. C. Morrison y R. G. Parr, Phys. Rev. A 50 (1994), 2138.

${ }^{40}$ O. V. Gritsenko, R. van Leeuwen y E. J. Baerendes, J. Chem. Phys. 104 (996), 8535; D. J. Tozer, V. E. Ingamells y N. C. Handy, J. Chem. Phys. 105 (1996), 9200.

${ }^{41}$ D. M. Ceperley y B. J. Alder, Phys. Rev. Lett 45 (1980), 566.

${ }^{42}$ S. J. Vosko, L. Wilk y M. Nussair, Can. J. Phys. 58 (8) (1980), 1200.

${ }^{43}$ A. D. Becke, Phys. Rev. B 38 (1988), 3098.

${ }^{44}$ C. Lee, W. Yang y R. G. Parr, Phys. Rev. B 37 (1988), 785. 
${ }^{45}$ J. P. Perdew, Phys. Rev. B 33 (1986), 8822; 34 (1986), 7406.

${ }^{46}$ J. P. Perdew, J. A. Chevary, S. H. Vosko, K. A. Jackson, M. R. Pederson, D. J. Singh y C. Fiolhais, Phys. Rev. B 46 (1992), 6671.

${ }^{47}$ A. D. Becke, J. Chem. Phys. 104 (1996) 1040.

${ }^{48}$ I. N. Levine, Química Cuántica $5^{\text {ta }}$ Edición, Prentice Hall (2001).

${ }^{49}$ A. D. Becke, J. Chem. Phys. 98 (1993), 5648.

${ }^{50}$ F. Jensen, Introduction to Computational Chemistry, John Wiley \& Sons Ltd. (1999).

${ }^{51}$ W. J. Hehre, R. F. Stewart y J. A. Pople, J. Chem. Phys. 51 (1969), 2657.

${ }^{52}$ J. S. Binkle y J. A. Pople, J. Am. Chem. Soc. 102 (1980), 939.

${ }^{53}$ W. J. Hehre, R. Ditchfield y J. A. Pople, J. Chem. Phys. 56 (1972), 2257.

${ }^{54}$ R. Krishnan, J. S. Binkley, R. Seeger y J. A. Pople, J. Chem. Phys 72 (1980), 650.

${ }^{55}$ T. H. Dunning Jr., J. Chem. Phys. 90 (1989), 1007; A. J. Wilson, T van Mourik y T. H. Dunning Jr., J. Mol. Struct. 388 (1996), 339.

${ }^{56}$ R. A. Kendall, T. H. Dunning Jr. y R. J. Harrison, J. Chem. Phys. 96 (1992), 6796.

57 P. Politzer y J.S. Murray, Molecular Electrostatic Potentials and Chemical Reactivity, en Reviews in Computational Chemistry, ed. Lipkowitz K. B. y Boyd D. B., VHC (1991).

${ }^{58}$ J. S. Murray y K. Sen ed., Rev. Comput. Chem. 2 (1996), 273.

${ }^{59}$ A. E. Reed, L. A. Curtiss y F. Weinholt, Chem. Rev. 88 (1988), 899.

${ }^{60}$ Gaussian 03, Revision D.01, M. J. Frisch, G. W. Trucks, H. B. Schlegel, G. E. Scuseria, M. A. Robb, J. R. Cheeseman, J. A. Montgomery, Jr., T. Vreven, K. N. Kudin, J. C. Burant, J. M. Millam, S. S. Iyengar, J. Tomasi, V. Barone, B. Mennucci, M. Cossi, G. Scalmani, N. Rega, G. A. Petersson, H. Nakatsuji, M. Hada, M. Ehara, K. Toyota, R. Fukuda, J. Hasegawa, M. Ishida, T. Nakajima, Y. Honda, O. Kitao, H. Nakai, M. Klene, X. Li, J. E. Knox, H. P. Hratchian, J. B. Cross, V. Bakken, C. Adamo, J. Jaramillo, R. Gomperts, R. E. Stratmann, O. Yazyev, A. J. Austin, R. Cammi, C. Pomelli, J. W. Ochterski, P. Y. Ayala, K. Morokuma, G. A. Voth, P. Salvador, J. J. Dannenberg, V. G. Zakrzewski, S. Dapprich, A. D. Daniels, M. C. Strain, O. Farkas, D. K. Malick, A. D. Rabuck, K. Raghavachari, J. B. Foresman, J. V. Ortiz, Q. Cui, A. G. Baboul, S. Clifford, J. Cioslowski, B. B. Stefanov, G. Liu, A. Liashenko, P. Piskorz, I. Komaromi, R. L. Martin, D. J. Fox, T. Keith, M. A. Al-Laham, C. Y. Peng, A. Nanayakkara, M. Challacombe, P. M. W. Gill, B. Johnson, W. Chen, M. W. Wong, C. Gonzalez, y J. A. Pople, Gaussian, Inc., Wallingford CT, 2004.

${ }^{61}$ R. S. Mulliken, J. Chem. Phys. 23 (1955) 1833 y J. Chem. Phys. 23 (1955) 1841.

${ }_{62} \mathrm{gOpenMol} \mathrm{http://www.csc.fi/english/pages/g0penMol}$

${ }^{63}$ B. H. Besler, K. M. Merz Jr. y P. A. Kollman, J. Comp. Chem. 11 (1990) 431; U. C. Singh y P. A. Kollman, J. Comp. Chem. 5 (1984) 129.

${ }^{64} \mathrm{http}: / /$ www.chem.wisc.edu/ nbo5/

${ }^{65}$ B.H. Boo y Z. Liu, J. Phys. Chem. A 103 (1999) 1250.

${ }^{66}$ S.K. Nayak, S.N. Khanna, y P. Jena, Phys. Rev. B 57 (1998) 3787.

${ }^{67}$ J.J. BelBruno, Chem. Phys. Lett. 313 (1999) 795.

${ }^{68}$ Y. Qu y X. Bian, Spect. Acta Part A 61 (2005) 1877.

${ }^{69}$ A. Costales, M. A. Blanco, A. M. Pendás, A. K. Kandalam y R. Pandey, J. Am. Chem. Soc. 124 (2002) 4116.

${ }^{70}$ A. K. Kandalam, R. Pandey, M. A. Blanco, A. Costales, J. M. Recio y J. M. Newsam, J. Phys. Chem. B 104 (2000) 4361.

${ }^{71}$ S. Burrill y F. Grein, J. Mol. Struc. (Theochem) 757 (2005) 137.

${ }^{72}$ L. Guo, H-s Wu y A-h Jin, J. Mol. Struc. (Theochem) 677 (2004) 102.

${ }^{73}$ B. Song y P-1 Cao, Phys. Rev. B 66 (2002) 033406:1.

${ }^{74}$ Ch. Chang, A. B. C. Patzer, E. Sedlmayr, T. Steinke y D. Sülzle, Chem. Phys. 271 (2001) 283.

${ }^{75}$ K. P. Huber, G. Herzberg; Constants of Diatomic Molecules; Van Nostrand: New York (1979).

${ }^{76}$ M. G. Giuffreda, M. S. Deleuze y J-P François, J. Phys. Chem. A 104 (2000), 5855 (y referencias allí incluidas).

${ }^{77}$ a- J. M. L. Martin, J-P J-P François y R. Gijbels, J. Chem. Phys. 90 (11) (1989), 6469; b- J. M. L. Martin, T. J. Lee, G. E. Scuseria y P. R. Taylor, J. Chem. Phys. 97 (9) (1992), 6549; c- J. M. L. Martin, J. El-Yazal y J-P François, Chem. Phys. Lett. 248 (1996), 95; d- J. M. L. Martin, J. El-Yazal, J-P François y R. Gijbels, Chem. Phys. Lett. 232 (1995), 289.

${ }^{78}$ H. Xu, C. U. Pittman Jr. y S. Saebo, J. Mol. Struct. (THEOCHEM) 621 (2003), 233.

${ }^{79}$ M. A. Mileev, S. M. Kuzmin y V. I. Parfenyuk, J. Struct. Chem. 47 (6) (2006), 1016.

${ }^{80}$ H. Bredohl, I. Dubois, Y. Houbrechts y P. Nzohabonayo, J. Phys. B: At. Mol. Phys. 17 (1984), 95.

${ }^{81}$ J. Zhao, L. Wang, J. Jia, X. Chen, X. Zhou y W. Lu, Chem. Phys. Lett., 443 (2007) 29 (y referencias allí incluidas).

${ }^{82}$ A. Tomasulo y M. V. Ramakrishna, J. Chem. Phys. 105 (23) (1996) 10449. 
${ }^{83}$ M. A. Al-Laham, G. W. Trucks y K. Raghavachari, J. Chem. Phys. 96 (1992) 1137; M. A. Al-Laham, G. W. Trucks y K. Raghavachari, J. Chem. Phys. 98 (1993) 8770.

${ }^{84}$ a- P. Y. Feng y K. Balasubramanian, J. Phys. Chem. A 103 (1999) 9093; b- K. Balasubramanian y P. Y. Feng, J. Phys. Chem. A 105 (2001) 11295.

${ }^{85}$ a- E. F. Archibong, R. M. Gregorius y S. A. Alexander, Chem. Phys. Lett. 321 (2000) 253; b- E. F. Archibong, A. St-Amant, S. K. Goh y D. Marynick, J. Phys. Chem. A 106 (2002) 5932.

${ }^{86}$ A. Costales, X. K. Kandalam, R. Franco y R. Pandey, J. Phys. Chem. B 106 (2002) 1940.

${ }^{87}$ Y. Qu y X. Bian, J. Comp. Chem. 26 (2004) 226.

${ }^{88}$ a- L. Guo, H. S. Wu y Z. H. Jin, J. Mol. Struct. (THEOCHEM) 648 (2004) 67; b- L. Guo, H. S. Wu y Z. H. Jin, In. J. Mass Spect. 240 (2005) 149.

${ }^{89}$ P. Karamanis y D. Xenides, Computation in Modern Science and Engeneering, Proceedings of the International Conference on Computational Methods in Science and Engineering 2007, editado por T. E. Simons ang G. Maroulis, Vol 2 Part A (2007) 68, American Institute of Physics; P. Karamanis y J. Leszczynski, J. Chem. Phys. 128 (2008) 154323.

${ }^{90}$ R. Linguerri, N. Komiha, R. Oswald, A. Mitrushchenkov y P. Rosmus, Chem. Phys. 346 (2008) 1.

${ }^{91}$ Y. Qu, W. Ma, X. Bian, H. Tang y W. Tian, Int. J. Quantum. Chem. 106 (2006) 960.

${ }^{92}$ R. Shi, J. Shao, C. Wang, X. Zhu y X. Lu, J. Mol. Model., DOI 10.1007/s00894-010-0801-x, in press.

${ }^{93}$ R. G. Parr, R. A. Donelly, M. Levy y W. E. Palke, J. Chem. Phys. 68 (1978) 3801.

${ }^{94}$ R. P. Iczkowski y J. L. Margrave, J. Am. Chem. Soc. 83 (1961) 3547.

95 J. P. Perdew, R. G. Parr, M. Levy y J. L. Balduz, Jr. Phys. Rev. Lett. 49 (1082) 1691; Y. Zang y W. Yang, Theor. Chem. Acc. 103 (2000) 346.

${ }^{96}$ R. S. Mulliken, J. Chem. Phys. 2 (1934) 782.

${ }^{97}$ L. Pauling, J. Am. Chem. Soc. 54 (1932) 3570.

${ }^{98}$ T. A. Koopmans, Physica 1 (1933) 104.

${ }^{99}$ W. Kohn, A. D. Becke y R. G. Parr, J. Phys. Chem. 100 (1996) 12974.

${ }^{100}$ T. T. Nguyen-Dang, R. F. W. Bader y H. Essén, Int. J. Quantum Chem. 22 (1982) 1049.

${ }^{101}$ R. G. Pearson, J. Am. Chem. Soc. 85 (1963) 3533.

102 J. Cioslowsky y M. Martinov, J. Chem. Phys. 101 (1994) 366.

${ }^{103}$ R. G. Parr y W. Yang, J. Am. Chem. Soc. 106 (1984) 4049.

${ }^{104}$ K. Fukui, Y. Yonezawa y H. Shingu, J. Chem. Phys. 20 (1952) 722; R. G. Parr y J. B. Parr, Theor. Chem. Acc. 102 (1999) 5; S. Kato, Theor. Chem. Acc. 103 (2000) 219.

${ }^{105}$ R. Balawander y L. Komorowsky, J. Chem. Phys. 109 (1998) 5203.

${ }^{106}$ P. Fuentealba, P. Pérez y R. Contreras, J. Chem. Phys. 113 (7) (200) 2544.

${ }^{107}$ A. D. Becke y K. E. Edgecombe, J. Chem. Phys. 92 (1990), 9.

${ }_{108}$ A. Savin, R. Nesper, S. Wengert y T. Fassler, Angew. Chem. Int. Ed. Engl. 36 (1997), 1808.

${ }^{109}$ B. Silvi y A. Savin, Nature 371 (1994), 683.

${ }^{110}$ S. Zoury, X. Krokidis, F. Fuster y B. Silvi, Comput. Chem., 23 (1999) 597. http://www.jussieu.fr/silvi

111 A. D. Becke y K. E. Edgecombe, J. Chem. Phys. 92 (1990), 5397.

${ }^{112}$ Y. Tal y R. F. W. Bader, Int. J. Quantum Chem. Quantum Chem. Symp. 12 (1978), 153.

${ }^{113}$ M. Kohout y A. Savin, Int. J. Quantum Chem. 18 (1997), 1431.

${ }^{114}$ C. Von Weizsacker, Z Phys 96 (1935), 431.

115 J. K. Burdett y T. A. McCornick, J. Phys. Chem. A102 (1998), 6366.

${ }^{116}$ R. F. W. Bader, Atoms in Molecules. A quantum Theory, Clarendon Press, Oxford 1990; R. F. W. Bader, Chem. Rev. 91 (1991), 287.

${ }^{117}$ C. L. Perrin, J. Am. Chem. Soc., 113 (1991), 2865.

${ }^{118}$ C. Mei, K. E. Edgecombe, V. H. Smith Jr y A. Heilingbrunner, Int. J. Quantum Chem., 48 (1993), 287.

${ }^{119}$ R. Hoffmann, Rev. Mod. Phys. (3) 60 (1988), 601.

${ }^{120}$ P. Geerlings, F. De Proft y W. Langenaeker, Chem. Rev. 103 (2003) 1793.

${ }^{121}$ R. Pis Diez y V. Ferraresi Curotto, Revista Aportes a las Ciencias Químicas 1 (1 y 2), (2008) 29. Editorial Científica Universitaria - UNCa.

${ }_{122}$ AIMPAC http://www.chemistry.mcmaster.ca/bader/aim/

${ }^{123}$ TopMod http://www.lct.jussieu.fr/pagesperso/silvi/manual/manual.html

${ }^{124}$ MullPop http://www.quimica.unlp.edu.ar/centros/cequinor/rpd sp.htm

${ }^{125}$ R. S. Mulliken, J. Chem. Phys. 23 (1955) 1833 y J. Chem. Phys. 23 (1955) 1841. 